\title{
Research Paper: Overcomeing Cognitive Impairments of Sleep-deprived Ovariectomized (OVX) Female Rats by Voluntary Exercise
}

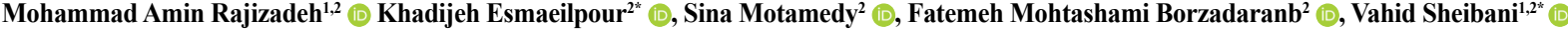

1. Department of Physiology, School of Medicine, Kerman University of Medical Sciences, Kerman, Iran.

2. Neuroscience Research Center, Institute of Neuropharmacology, Kerman University of Medical Sciences, Kerman, Iran.

\begin{tabular}{|c|c|}
\hline $\begin{array}{l}\text { Use vour device to tocan } \\
\text { and read the article online }\end{array}$ & \\
\hline $\lim _{10}$ & $\begin{array}{l}\text { ditation: Rajizadeh, M. A., Esmaeilpour, Kh., Motamedy, S., Mohtashami Borzadaranb, F., \& Sheibani, V. (2020). Cognitive } \\
\text { Impairments of Sleep-Deprived Ovariectomized (OVX) Female Rats by Voluntary Exercise. Basic and Clinical Neuroscience, } \\
\text { 11(5), 573-586. http://dx.doi.org/10.32598/bcn.9.10.505 }\end{array}$ \\
\hline 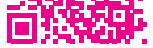 & dof) http://dx.doi.org/10.32598/ben.9.10.505 \\
\hline
\end{tabular}

Article info:

Received: 11 Aug 2019

First Revision: 25 Aug 2019

Accepted: 08 Nov 2019

Available Online: 01 Sep 2020

Keywords:

Sleep Deprivation (SD), Voluntary exercise, Ovariectomized (OVX) female rat, Learning and memory, Anxiety-like behavior, Corticosterone

\section{A B S T RA C T}

Introduction: Previous studies demonstrated that forced and voluntary exercise had ameliorative effects on behavioral tasks followed by Sleep Deprivation (SD) in intact female rats. The main goal of this research was evaluating the impact of voluntary exercise on cognitive functions while SD and ovariectomization is induced in female wistar rats.

Methods: The rats were anesthesized combining dosage of ketamine and xylazine. Then, both ovaries were eliminated and 3 weeks after surgery the animals entered the study. The exercise protocol took 4 weeks of voluntary exercise in a wheel which was connected to home cage. For inducing a 72 hours deprivation the multiple platforms was applied. The cognitive functions were studied by exploiting the Morris Water Maze (MWM) and Novel object recognition tests. Anxiety was evaluated by open field test and corticostrone measurement was carried out by ELISA method. One-way and two-way ANOVA and repeated measures were utilized for data analysis and $\mathrm{P}<0.05$ was considered statistically significant.

Results: We observed significant spatial and recognition learning and memory impairments in OVX sleep-deprived rats compared to the control group and voluntary exercise alleviated the SD-induced learning and memory defects.

Conclusion: We concluded that voluntary exercise can improve cognitive impairments followed by SD in OVX female rats.

\section{* Corresponding Author:}

Khadijeh Esmaeilpour, PhD.

Address: Neuroscience Research Center, Institute of Neuropharmacology, Kerman University of Medical Sciences, Kerman, Iran. Tel: +98 (343) 2264196

E-mail: esmaeilpour@kmu.ac.ir
* Corresponding Author: Vahid Sheibani, PhD.

Address: Department of Physiology, School of Medicine, Kerman University of Medical Sciences, Kerman, Iran.

Tel: +98 (343) 2264196

E-mail:vsheibani2@yahoo.com 


\section{Highlights}

- Sleep Deprivation (SD) impaired learning and memory in OVX female rats.

- Voluntary exercise alleviated the SD-induced cognitive impairments in OVX female rats.

- There was no significant change in the plasma corticosterone level of all groups.

\section{Plain Language Summary}

WR and TR are two widely used chronic exercise models. TR is a form of forced exercise and WR is a form of voluntary exercise. Both exercise paradigms have exhibited different effects on anxiety-like behaviors. Human and animal studies show the important role of sleep in certain types of learning and memory as well as neuronal plasticity. Recent studies have indicated that single night or mild Sleep Deprivation (SD) can cause disruptions in various forms of memory. In this study the role of voluntary exercise on cognitive impairments followed by SD in Ovariectomized (OVX) adult female rats is evaluated using behavioral studies

\section{Introduction}

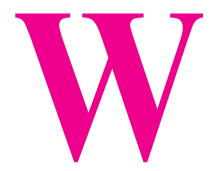

heel Running (WR) and Treadmill Running (TR) are two of the widely-used chronic exercise paradigms in rodent models. TR is considered as a form of forced exercise with fixed parameters such as intensity, duration, and so on while WR is a form of voluntary exercise with less-characterization of parameters in exercise performance (Lin et al., 2012). WR gives the animal the freedom to choose the dosage and the timing of the exercise. On the other hand, forced exercise eliminates the freedom aspect and is usually contingent on an aversive stimulus for behavioral conditioning. In a treadmill, this aversive stimulus is electrical shock (Landers, Kinney, Allen, \& van Breukelen, 2013). While the aversive stimulus is known to be effective at conditioning running behavior, it can also lead to stress in the animal (Landers et al., 2013). Voluntary running is done when the animal decides, which normally takes place in the dark cycle whereas, forced running usually happens during the light cycle for convenience (Landers et al., 2013). Rodents are nocturnal animals and less activity is observed during the light cycle and so it is plausible that this may add to the stress they already experience in the forced running (Landers et al., 2013).

Studies with both exercise paradigms have exhibited different effects on anxiety-like behaviors (Burghardt, Fulk, Hand, \& Wilson, 2004; Dishman, 1997; Leasure \& Jones, 2008), neurogenesis (Leasure \& Jones, 2008), and hippocampal expression of various neural-active molecules (Dishman, 1997; Ploughman et al., 2005).
But, either treadmill exercise or wheel running has led to improvements in hippocampus-dependent spatial learning and memory (Liu et al., 2009).

Evidence-based studies on humans and animals show the critical role of sleep in certain types of learning and memory as well as neuronal plasticity (Diekelmann \& Born, 2010). A famous hypothesis is that post-training sleep facilitates the consolidation of new information from short-term into long-term memory (Walker \& Stickgold, 2004). Another hypothesis states that memory trace reactivation and consolidation might take place during sleep (Lee \& Wilson, 2002). Several clinical studies have demonstrated that anxiety symptoms are observed in the absence of periods of sleep (Silva et al., 2004; Vollert et al., 2011). Recent studies have indicated that single night or mild Sleep Deprivation (SD) can cause disruptions in various forms of memory, including procedural, implicit, and working memory in humans (Forest \& Godbout, 2000; Yoo, Hu, Gujar, Jolesz, \& Walker, 2007). There have been reports that one to five days of SD, before executing a cognitive task, has an impact on behavioral efficiency in rodents (Alvarenga et al., 2004; Silva et al., 2004). In other studies, SD was shown to have deleterious effects on Morris Water Maze (MWM) (Hajali, Sheibani, Esmaeili-Mahani, \& Shabani, 2012; Rajizadeh et al., 2018; Salari et al., 2015), open field (Hajali et al., 2012) and novel objective recognition (Binder et al., 2012; Palchykova, Winsky-Sommerer, Meerlo, Dürr, \& Tobler, 2006; Rajizadeh et al., 2018). Some studies show that female rats are more vulnerable to deleterious effects of SD than male rats (Hajali et al., 2012). 


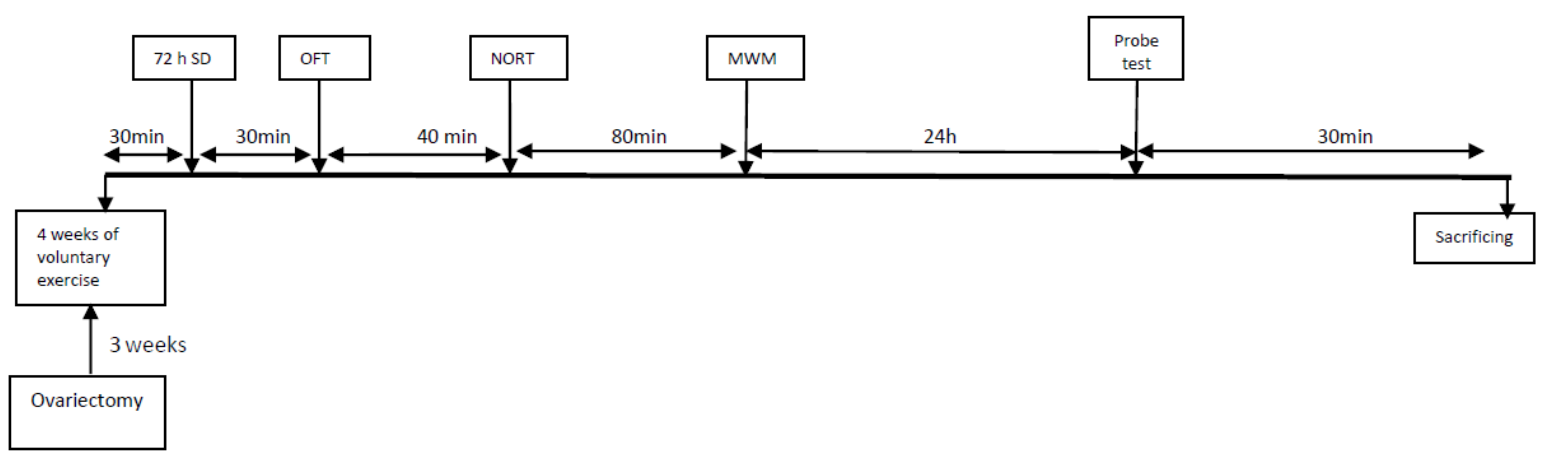

NEUR SCIENCE

Figure 1. Time-line diagram showing the protocol used for voluntary exercise application

In animals of SD/exercise group; also showing the timing of behavioral tasks.

Female hormones such as steroid hormones, estradiol, and progesterone affect cognitive performance as well as modulating structural, biochemical, and electrical activity in the hippocampus (Brinton, 2009; Foy, Baudry, Akopian, \& Thompson, 2010).

The effect of sleep deficiency on cognitive performances has mostly been studied during adulthood in both human and animal studies and very little data exist concerning the effects of poor sleep in gonadal hormone-depleted status (e.g. postmenopausal women or OVX animal models) (Henderson \& Sherwin, 2007; Mitchell \& Woods, 2011).

Previous publications have demonstrated that forced exercise can ameliorate cognitive impairments followed by SD (Saadati et al., 2015; Salari et al., 2015) and also we showed in a previous study that voluntary exercise improved cognitive impairments due to SD in intact female rats (Rajizadeh et al., 2018). In this study, we evaluated the role of voluntary exercise on cognitive impairments followed by SD in ovariectomized (OVX) adult female rats using behavioral studies.

\section{Materials and Methods}

\subsection{Animals}

All experimental protocols were carried out according to the guidelines approved by the Ethics Committee of Kerman Neuroscience Research Center. We tried to reduce the discomfort for the animals during our experiments based on (Ethics Code: KNRC-95-30). Adult female Wistar rats with a weight range of 200-250 g were chosen and divided into seven groups. The animals were put into cages with free access to food and water. The temperature was fixed at $23 \pm 1^{\circ} \mathrm{C}$ and the rats were under
12-12 h light-dark cycle (lights on: 07:00-19:00). A total of 42 Ovariectomized (OVX) rats were assigned to subgroups randomly. The first group was the control group. The OVX group was maintained in home cages, the second group was Sleep-Deprived (SD). The third one was labeled as a wide platform (Sham platform) and it could sleep. A wide platform was utilized to understand the effects of the environment on this group. The fourth group was the exercise group with 4 weeks of voluntary exercise. The fifth and sixth groups were wide platform/exercise and exercise/SD, respectively. The seventh group was a separate group of rats known as the sham ovariectomized (Sham.OVX) group that underwent surgery without removing the ovaries. The aim of having a sham surgery group was to understand the effects of surgical stress. All OVX and sham surgery groups were put under ovariectomy surgical procedure. The number of rats in each group was 7 (Figure 1).

\subsection{Surgical procedures}

By applying a mixture of xylazine and ketamine with a dosage of and $10 \mathrm{mg} / \mathrm{kg}$, IP xylazine, and $60 \mathrm{mg} / \mathrm{kg}$, IP ketamine, general anesthesia was induced and surgical operations were performed. Under aseptic conditions, both ovaries of the rats were discarded through a small incision in the mid-abdomen. After the operation, all rats were housed for three weeks in the animal room (Ben et al., 2010).

\subsection{Exercise protocol}

In the wheel exercise group, the rats were put in home cages individually, which was connected to a running wheel (diameter, $20 \mathrm{~cm}$; width, $9 \mathrm{~cm}$ ) with a duration of $24 \mathrm{~h}$ for 4 weeks. The number of revolutions as well as and wheel revolutions was counted. The counting was not dependent on the wheel's direction. All the rats were 

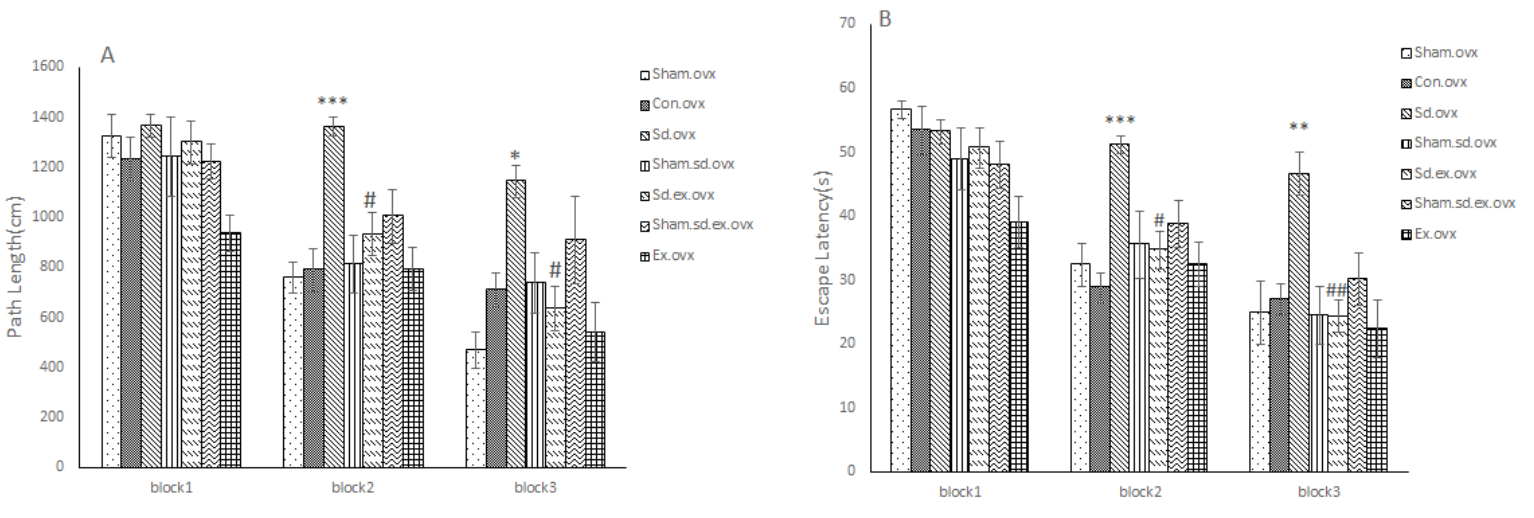

NEUR:SCIENCE

Figure 2. The effects of Sleep Deprivation (SD) and voluntary exercise on spatial learning in the MWM task

A: Sleep deprivation resulted in a significant increase of the traveled distance; and B: Escape latency to find the hidden platform compared to the control group. The application of voluntary exercise in the SD group significantly decreased the distance and time to find the hidden platform in blocks compared to those in the SD group. Two-way ANOVA and repeated measures were used for the analysis of these data;

Mean $\pm \mathrm{SD},{ }^{*} \mathrm{P}<0.05$ vs control, \# $\mathrm{P}<0.05$ vs SD, *** $\mathrm{P}<0.001$ vs control, \#\#\# $\mathrm{P}<0.001$ vs SD, ** $\mathrm{P}<0.01$ vs control, \#\# $\mathrm{P}<0.01$ vs SD

given the chance of habituation to the experimental environment and this activity took place 30 min per session in 2 days before starting the exercise protocol. This habituation was to minimize nonspecific stress responses (O’Dell et al., 2007; Sheibani, Rafie, Shahbazi, Naghdi, \& Sheikh, 2017). About $30 \mathrm{~min}$ after the last exercise session, SD began. In the exercise group, the average exercise in 4 weeks was $21563.82 \mathrm{~m}$ among all the rats, and in the exercise/SD group, the average exercise was $25459.96 \mathrm{~m}$.

\subsection{Induction of Sleep Deprivation (SD)}

A multiple platform device was utilized for inducing SD. The apparatus dimensions were $90 \times 50 \times 50 \mathrm{~cm}$ and it also contained 10 columns. The columns were $10 \mathrm{~cm}$ high, with $7 \mathrm{~cm}$ diameter, and placed $2 \mathrm{~cm}$ above the surface of the water, in two rows. These rows were organized $10 \mathrm{~cm}$ apart in an edge to edge manner, which gave the rats enough freedom to jump from one platform to another. Because of maintaining social stability, in this method, 4 rats were settled together in the same cage. The rats could freely access food pellet baskets which were always provided as well as clean water bottles. Food pallets and water bottles were positioned on the top of the cage. In this research, the duration of SD was $72 \mathrm{~h}$ (Salari et al., 2015). SD paradigm was done $30 \mathrm{~min}$ following the last exercise session in the exercise/SD groups in the course of SD interval, with a duration of $72 \mathrm{~h}$ at a temperature of $23+1^{\circ} \mathrm{C}$. Light/dark cycles were both kept under controlled conditions. The possibility of stress in a novel environment was examined by putting the rats of the control group in a similar chamber with wider platforms. This platform is known as a sham platform which is $10 \mathrm{~cm}$ high and $15 \mathrm{~cm}$ in diameter. It was designed big enough so the rats could sleep without falling into the water (Hajali,Sheibani, Esmaeili-Mahani, \& Shabani, 2012; Rajizadeh et al., 2018).

\subsection{Morris Water Maze (MWM)}

MWM is a black circular pool with a height of $80 \mathrm{~cm}$ and a diameter of $160 \mathrm{~cm}$. The depth of water in the pool is $40 \mathrm{~cm}$. The necessary technology is provided to maintain the water at room temperature. The circular-shaped pool is sectioned into four quadrants of equal size. Each quadrant was marked as $\mathrm{N}, \mathrm{S}, \mathrm{E}$, and $\mathrm{W}$ to show the starting points. A square platform with a $10 \mathrm{~cm}$ diameter, positioned in the center of the northeast quadrant. This platform was hidden at $1.5 \mathrm{~cm}$ beneath the water surface. The MWM is located in a dimly lit room. Diverse extra geometric images such as circles, squares, or triangles were used as cues. These images were attached to various positions on the walls around the maze and the object positions were kept intact during the whole experimental procedure. A smart video tracking system (Noldus Ethovision ${ }^{\circledR}$ system, version 7, Netherlands) was installed to record the performance, and animals were monitored and tracked on the screen of a computer (Hajali et al., 2012). Also, the temperature of the water was fixed at $25 \pm 2^{\circ} \mathrm{C}$. 


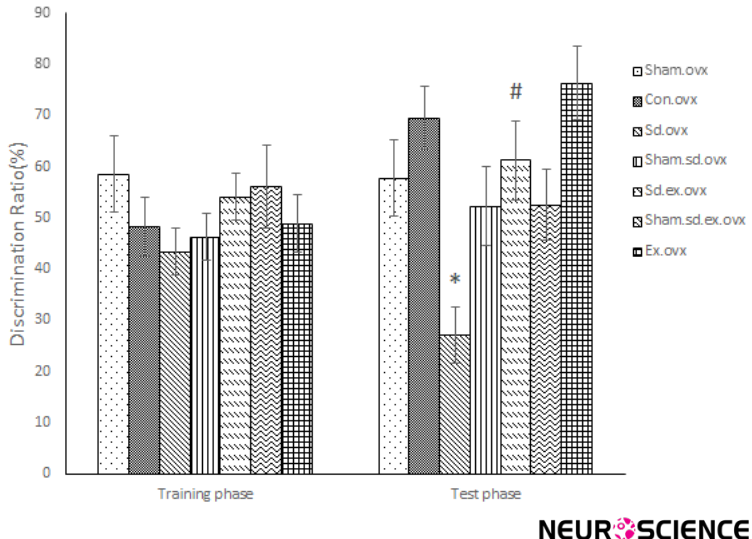

Figure 3. The effects of Sleep Deprivation (SD) and voluntary exercise on spatial memory in the MWM task

Sleep Deprivation (SD) resulted in a significant decrease in the percentage of time spent and distance traveled in the target quadrant and the number of crossing from the platform region compared to the control group. Animals of the exercise/SD group significantly showed an increase in these parameters compared to the SD group;

One-way ANOVA was used for the analysis of these data;

Mean \pm SEM; ** $\mathrm{P}<0.01$ vs control; \# $\mathrm{P}<0.05$ vs SD

\subsection{Spatial learning and memory}

In a single training protocol, each rat managed to accomplish three blocks. These blocks were subject to a 30-min rest interval. A block was designed with four consecutive trials which had a duration of $60 \mathrm{~s}$ and the inter-trial intervals of $60 \mathrm{~s}$. All of the experiments on the rats were carried out after finishing the SD period. Based on the protocol, the tests could only be done between 9:30 and 12:30 AM. On each trial, starting from one of the four quadrants of the maze, the rats were released into the water in a random manner facing the wall of the pool. Each rat experienced 4 different releasing locations. During the phase of acquisition, the platform location was kept intact and rats were given 60 seconds to swim to the hidden escape platform. After the animals discovered the platform, they were given 20-30 seconds to remain on the platform. Then, they were put in a cage to wait for 20-30 s before the start of the next trial. If a rat didn't manage to find the platform in $60 \mathrm{~s}$, it was gently led toward the platform by the experimenter and after 20-30 s staying on the platform. The rats were put in an animal cage to wait for 20-30 s before the start of the next trial. The data related to the time and distance of finding the platform were gathered for later analysis. After the last training trial, the memory retention test was performed 24 hours later. The memory test was a single probe trial and was performed to examine the spatial memory in the water maze following sleep deprivation.

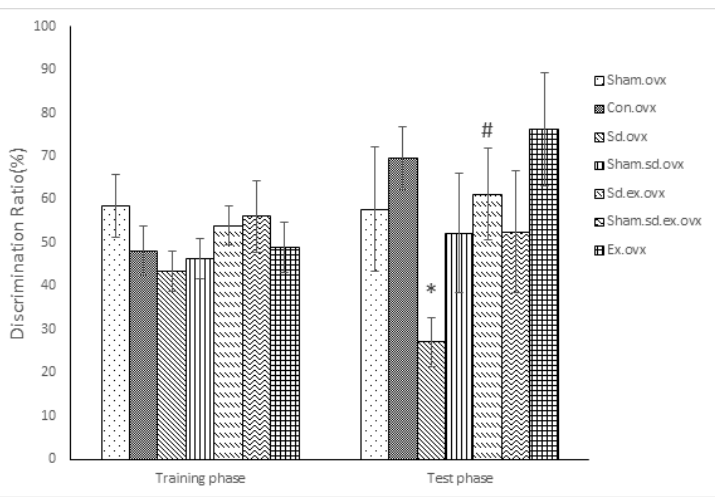

NEUR SCIENCE

Figure 4. The effects of sleep deprivation (SD) and voluntary exercise on the novel object recognition test

There was no significant difference between all groups regarding the exploration time in the training session. There was a significant decrease in exploration time in a test session in the SD group compared to the control group and the application of voluntary exercise in the exercise/SD group increased exploration time compared to the SD group. Oneway ANOVA was used for statistical analysis. Mean \pm SD.* $\mathrm{P}<0.05$ vs control. \# $\mathrm{P}<0.05$ vs SD.

In this trial, the experimenter removed the platform, and the rat was allowed to swim for 60 seconds. The time and distance percentage spent in the target quadrant (quadrant 4) were analyzed and considered as an indicator of spatial memory retention. After the probe trial, a visible platform test was performed to determine any possibility of SD interference with sensory and motor coordination or motivation. In this stage, the animals were tested for their ability to find a visible platform. The platform was placed $2 \mathrm{~cm}$ above the water level and was visible with aluminum foil. It should be mentioned that during the procedure, the experimenter remained blind to the rats' groups (Rajizadeh et al., 2018; Rajizadeh et al., 2019; Salari et al., 2015). About $160 \mathrm{~min}$ after SD, MWM took place and all 3 blocks were accomplished on the same day and the probe test was done $24 \mathrm{~h}$ after SD.

\subsection{Open Field test}

The open field test is performed to evaluate the possible impact of SD on anxiety-like behaviors and locomotion. The square-shaped arena is opaque Plexiglas with the dimensions of $90 \times 90 \times 45 \mathrm{~cm}$. This arena is also sectioned into 16 small squares so that the rats spend time in either central or peripheral squares.

In the current study, the rats were positioned in the middle of the arena. An automated video tracking system (Ethovision, version 7, Noldus Technology, Netherlands) 

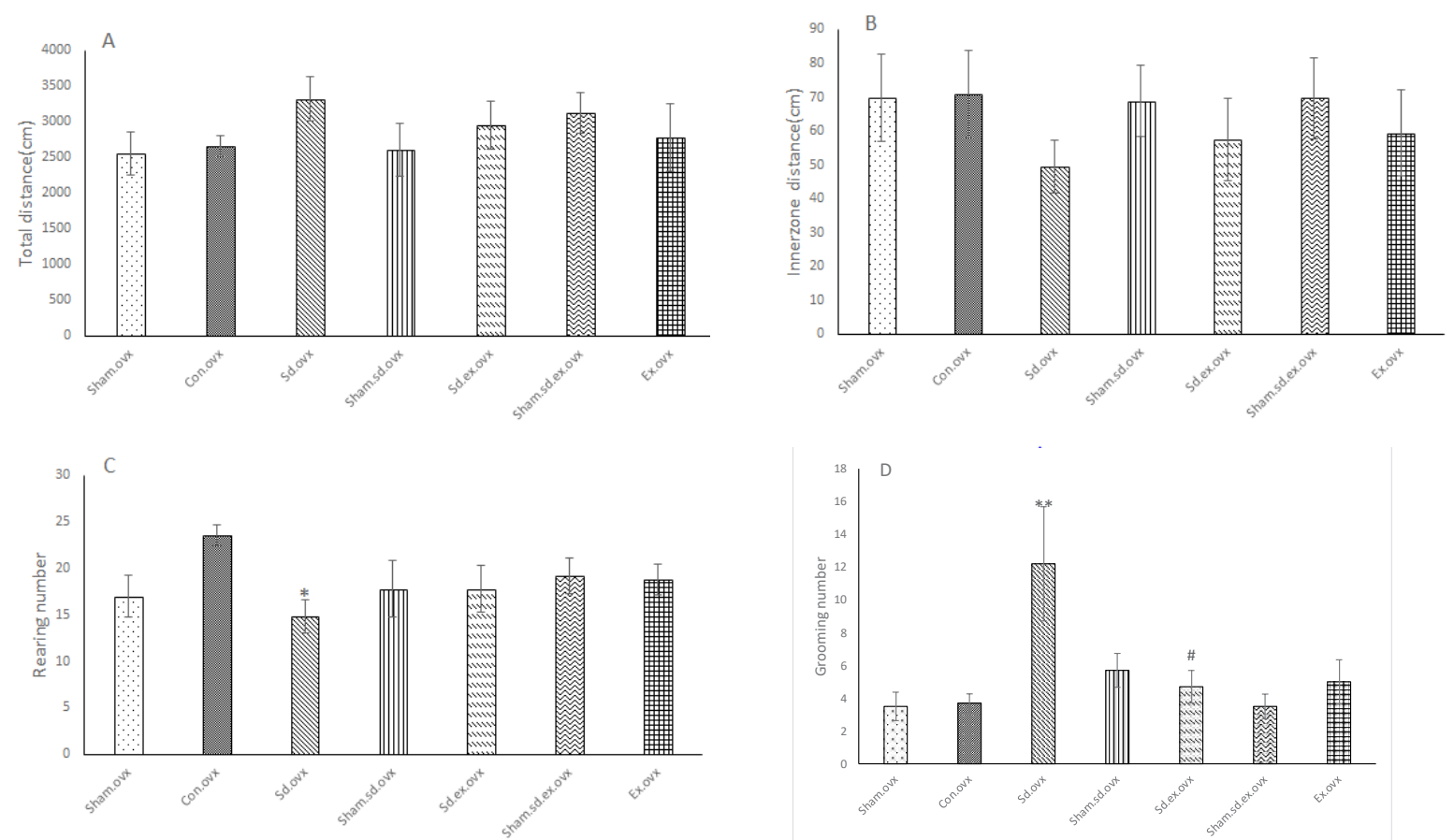

NEURSCIENCE

Figure 5. The effects of Sleep Deprivation (SD) and voluntary exercise on the anxiety-like behavior and exploratory activity in the open field test

A and B: Total distance and distance moved inner zone had no significant difference among all groups; C: Rearing number; $\mathrm{D}$ : Decreased and grooming frequency increased in the SD group compared to other groups. One-way ANOVA was used for analyzing these results. Mean $\pm \mathrm{SD}, * \mathrm{P}<0.05$ vs control, ${ }^{* *} \mathrm{P}<0.01$ vs control, ${ }^{*} \mathrm{P}<0.01$ vs SD.

recorded and analyzed their behavior during a 5-min interval. The recorded parameters for each rat were the total distance moved, the total time spent in the center or periphery, speed, and the number of grooming and rearing (Rajizadeh et al., 2018; Vaziri et al., 2015). the open field test was carried out exactly 30 min after SD.

\subsection{Novel Object Recognition test}

In a 10-min habituation session, all animals were given time to explore a plastic cage $(60 \times 60 \times 40 \mathrm{~cm})$. In this phase, no object was presented and the cage was equally illuminated. About 30 minutes after the habituation period, the animals were given the freedom to explore two identical objects placed at the same position inside the box for a total of $5 \mathrm{~min}$ (training session). A memory retention ability test was performed $45 \mathrm{~min}$ after the training session. During a 3-min test session, the animals were exposed to familiar and novel objects placed in the same position that the objects were in the training phase. However, during the test phase, the location of the new object was pseudo-randomly changed to avoid the natural preference of animals for a certain location. All the presented objects (available in duplicate) were similar in material and size but distinct in shape. After each animal, the objects were cleaned and then air-dried using a $70 \%$ alcohol box. The exploration time was defined as the duration of sniffing or touching the object with the nose quantified by a camera. Finally, discrimination ratio (as recognition index) is defined as the ratio of time spent for exploring each object divided by the total time spent for exploring both objects multiplied by 100 in training and test phases (Esmaeilpour, Sheibani, Shabani, \& Mirnajafi-Zadeh, 2017; Rajizadeh et al., 2018). The test was carried out approximately $80 \mathrm{~min}$ after SD.

\subsection{Plasma corticosterone measurement}

The effect of voluntary exercise and SD on corticosterone levels was evaluated in all animals. All procedures were carried out between 9:10 and 9:30 AM (30 min after the end of the probe test in MWM). Using $\mathrm{CO} 2$, the animals were anesthetized. We took trunk blood using plastic polyethylene tubes on ice containing Na2EDTA as an anticoagulant, immediately after decapitation. The blood was centrifuged at $2600 \mathrm{rpm}$, for $20 \mathrm{~min}$ at a temperature of $4^{\circ} \mathrm{C}$. The plasma was collected into micro tubes and refrigerated at $-80^{\circ} \mathrm{C}$ for analyzing the corticosterone levels in all of the samples. The plasma sample analysis was carried out by an ELISA kit which 
is designed for rats and mice. This kit is specifically for someone who is blind to the treatment of the animals (Rajizadeh et al., 2018; Saadati et al., 2015).

\subsection{Data analysis}

During the acquisition phase, the time spent and the distance traversed by rats to find the hidden platform in the MWM training were analyzed. The analysis was carried out by a two-way Analysis of Variance (ANOVA) parallel with repeated measures. Through this method, the differences in the learning rates among groups (group and block as the factors) were determined. Data analysis related to the collected data from the MWM probe trials, swim speed, and corticosterone levels, and novel objective and open field data were done by a One-way Analysis of Variance (ANOVA). Tukey's post hoc multiple comparison test was performed to determine points of a significant difference since statistical significance was seen among groups. The data were expressed as Means \pm SEM, and $\mathrm{P}<0.05$ was considered statistically significant (Saadati et al., 2015).

\section{Results}

3.1. Effects of sleep deprivation and voluntary exercise on spatial learning and memory

Animals in all groups managed to learn the platform hidden underwater and this learning was done during the acquisition phase. Declined swimming distance and escape latency across blocks of training is considered as evidence for learning (Figure $2 \mathrm{~A} \& \mathrm{~B}$ ).

Two- way analysis of ANOVA was performed and it was observed that the distance and escape latency of the Sleep-Deprived group (SD) increased significantly in block 2 (Figure 2A; $\mathrm{P}<0.001 \&$ Figure 2B; $\mathrm{P}<0.001$ ) and block 3 (Figure 2A; $\mathrm{P}<0.05$ \& Figure 2B; $\mathrm{P}<0.01$ ) in comparison to the control group. Sleep-deprived rats showed better potential in finding the hidden platform and it was improved significantly in a 4-week running wheel exercise. This was seen by observing the significant decrease in their distance of swimming in block 2 (Figure 2A; $\mathrm{P}<0.05$ ) and block 3 (Figure 2A; $\mathrm{P}<0.05$ ) and a significant decline in the escape latency in block 2 (Figure 2B; $\mathrm{P}<0.05$ ) and block 3 Figure 2B; $\mathrm{P}<0.01$ ) compared to the SD group in the MWM test.

No significant difference was observed in swimming speed and escape latency to find the visible platform among all the groups (Table 1). Therefore, SD did not affect visual and motor function.

Twenty-four hours after the acquisition phase, the probe test was performed to measure long-term retention of spatial memory after sleep deprivation. The obtained results consist of the distance, the number of crossing in the target quadrant, and the mean percentage (\%) for time. The probe test results proved less memory retention in sleep-deprived rats. The sleep-deprived rats spent less time and distance in the desired quadrant compared to the control group (Figure 3: $\mathrm{P}<0.01$ for time; Figure 3: $\mathrm{P}<0.01$ for distance and Figure 3: $\mathrm{P}<0.01$ for crossing) which was an indication of long-term memory impairment. However, during 4 weeks of wheel running exercise in the exercise/sleep-deprived groups, this impairment was significantly prevented since more time was spent in the target quadrant and longer distance was traversed in the target quadrant compared to the sleepdeprived rats (Figure 3: $\mathrm{P}<0.05$ for time \& Figure 3: $\mathrm{P}<0.05$ for distance and Figure 3: $\mathrm{P}<0.05$ for crossing) (One-way ANOVA followed by Tukey's test).

Table 1. Comparisons of swimming speed and latency to escape into the visible platform in Morris water maze

\begin{tabular}{ccc}
\hline Groups & Swimimig Speed $(\mathbf{c m} / \mathbf{s})$ & Escape Latecy to Visibe Platform $(\mathbf{s})$ \\
\hline Sham.ovx & $29.46 \pm 1.53$ & $15.7 \pm 0.75$ \\
\hline Con.ovx & $26.19 \pm 0.86$ & $15.3 \pm 0.59$ \\
\hline Sd.ovx & $24.7 \pm 1.12$ & $14.16 \pm 0.73$ \\
\hline Sham.sd.ovx & $28.82 \pm 2.19$ & $14.27 \pm 0.55$ \\
\hline Sd.ex.ovx & $27.62 \pm 1.73$ & $15.64 \pm 0.67$ \\
\hline Sham.SD.ex.ovx & $26.85 \pm 2.15$ & $15.75 \pm 0.72$ \\
\hline Ex.ovx & $23.74 \pm 1.43$ & $15.45 \pm 0.91$ \\
\hline
\end{tabular}

The differences were not significant; Data are presented as Mean \pm SEM (7 rats / group)

NEUR SCIENCE 


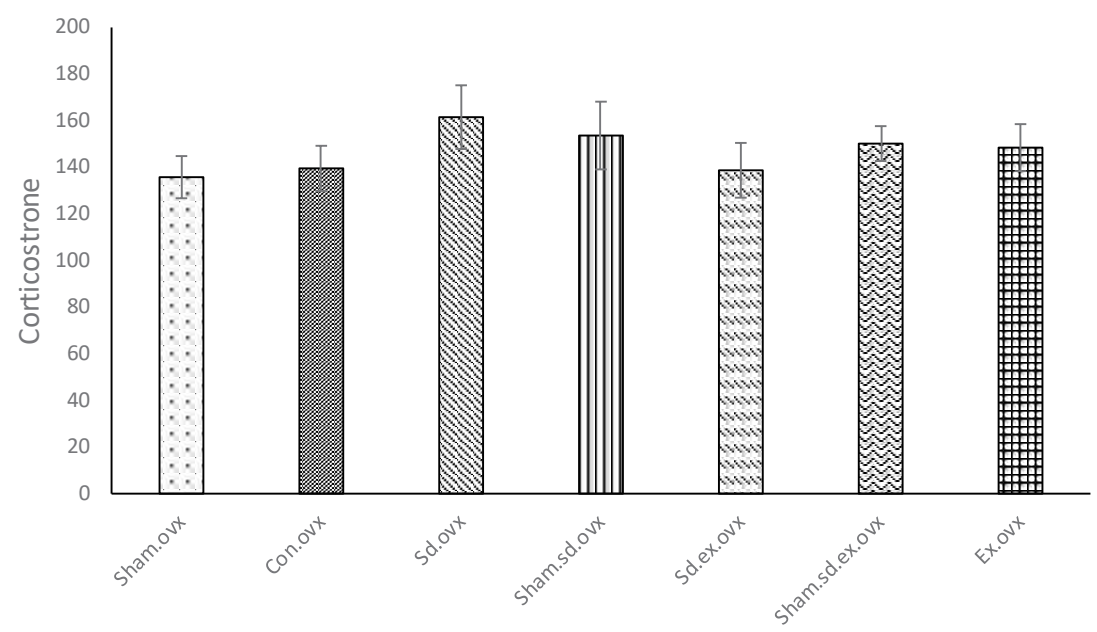

NEUR SCIENCE

Figure 6. The effects of Sleep Deprivation and voluntary exercise on corticosterone level

There was no significant difference between all groups regarding blood corticosterone levels. Analysis of data was done by One-way ANOVA. Data are presented as Means \pm SEM.

\subsection{Effect of sleep deprivation and voluntary exer- cise on the novel objective recognition test}

In the first trial, two familiar objects were presented in the arena and all groups spent a similar amount of time for object exploration and there were no significant differences between all groups (Figure 4). In the second trial, an assessment of object recognition memory was done by replacing the first trial objects with a copy of the original object. The novel object recognition memory was evaluated as the preference for exploring the novel object. In SD rats, significant disruption in new object recognition memory was observed. SD rats devoted less time for investigating the novel objects in comparison with the familiar objects and significant bias was not observed towards the novel objects. In these animals, the discrimination ratio was significantly lower than that in the control group (Figure 4: $\mathrm{P}<0.05$ ). Four weeks of wheel running exercise helped ameliorate the impairments induced by SD when a novel objective recognition test was implemented (Figure 4: $\mathrm{P}<0.05$ ) (One-way ANOVA followed by Tukey's test).

3.3. The effect of sleep deprivation and voluntary exercise on explorative and anxiety-like behaviors

No significant difference in total distance was seen in rats of the SD group in comparison with other groups. No significant differences were seen between all groups in the distance moved in the center in the open field test (Figure 5A \& 5B). Rearing numbers were declined significantly in the SD group in comparison with the control group (Figure 5C: $\mathrm{P}<0.05$ ) and grooming frequency was notably enhanced in the sleep-deprived group compared to the control group (Figure 5D: $\mathrm{P}<0.01$ ).

Four weeks of wheel running exercise could lead to a rise in the rearing numbers in the sleep-deprived rats but this increase can not be considered as an outstanding value. Also, it significantly decreased the grooming frequency in sleep-deprived rats compared to the sleepdeprived group (Figure 5D: $\mathrm{P}<0.05$ ) (One-way ANOVA followed by Tukey's test).

\subsection{Effects of Sleep Deprivation (SD) and volun- tary exercise on corticosterone level}

Our data showed no significant difference in corticosterone levels between all study groups (Figure 6).

\section{Discussion}

This study has focused on investigating the impact of wheel running exercise in 4 weeks on learning and memory as well as anxiety-like behaviors followed by $72 \mathrm{~h} \mathrm{SD}$ in female rats that were ovariectomized. Our results disclosed that $\mathrm{SD}$ alone weakens spatial learning in the OVX female rats. It can also disrupt the long-term spatial memory and novel object recognition memory in them. It also seems that voluntary exercise has a protective effect on the OVX rats against the stated negative effects. Also, our results disclosed that SD and voluntary exercise could change anxiety-like behaviors, but these changes were not significantly observed in blood corticosterone levels. In this research, our data revealed that 
SD had no major effect on voluntary locomotion compared to other groups in OVX female rats.

Sleep deprivation was induced via the multiple platform method. In this method, muscle tone is lost during the period of REM (rapid eye movement) sleep. This technique leads to suppressing about $95 \%$ of REM sleep and one can also observe interference with NREM (non-rapid eye movement) sleep (Machado, Hipolide, Benedito-Silva, \& Tufik, 2004). Thus, in this research, sleep deprivation in female rats was induced using multiple platform devices. This protocol can omit restraint factors and social isolation. One of these restraint factors is immobilization which is accompanied by other approaches (Suchecki \& Tufik, 2000). Also, previous data in our laboratory did not exhibit significant differences between corticosterone levels in SD and exercise groups compared to the control and sham platform (wide platform) groups (Saadati et al., 2015). Besides, the results of the current study indicated that both control and sham platform groups exhibit similarities in spatial memory test (MWM). One can claim that the cognitive impairment in sleep-deprived rats on the narrow platforms was a consequence of sleep loss and not the stress induced by the column in the water apparatus. Besides, the changes observed in the sleep-deprived rats were not a consequence of motor and visual impairments. This claim could be justified by approximately equal swimming velocity and latency to reach the visible platform in our groups.

Evidence supports that learning and memory in the MWM task can be improved by physical exercise (Aguiar et al., 2011; Marlatt, Potter, Lucassen, \& van Praag, 2012). Based on evidence from previous research, the beneficial effects of physical exercise on SD-induced memory impairments in male rats can be supported (Zagaar et al., 2012). Exercise can be an ameliorating measure against observed memory impairments in a range of neurodegenerative diseases (Hoveida, Alaei, Oryan, Parivar, \& Reisi, 2011). Physical exercise can induce changes in some forms of synaptic plasticity such as LTP (long term potentiation) (Cotman \& Berchtold, 2002). Previous data have demonstrated that exercise can alter some neurotransmitters as well as neurotrophic expression in the brain (Cotman \& Berchtold, 2002).

Generally, SD negatively affects learning and memory and it seems that exercise plays a protective role for rats against these negative effects (Salari et al., 2015). According to the evidence, one can claim that exercise is one of the potential non-pharmacological interventions that can improve cognitive functions (Shangold, 1990). Furthermore, research has indicated that exercise improves memory function during estrogen deficiency (Ben et al., 2010).
The impact of female sex steroids is clear in regulating sleep in postmenopausal women with low levels of the circulating estrogen (Dzaja et al., 2005; Manber \& Armitage, 1999) and they tend to be more vulnerable to the deleterious effects of poor sleep on cognitive performance (Hajali et al., 2012). Albeit, human studies have claimed that differences in sleep, mood, and public health among menopausal and premenopausal women are not significant (Manber \& Armitage, 1999). However, various studies on human subjects have reported that postmenopausal women are more vulnerable to deleterious effects of poor sleep on cognitive performance (Dzaja et al., 2005; Manber \& Armitage, 1999; Paul, Turek, \& Kryger, 2008). Hormone elevations in the ovaries are related to increased learning and memory (Gasbarri, Tavares, Rodrigues, Tomaz, \& Pompili, 2012). Furthermore, female rodents in proestrus/estrus performed better compared to females in metestrus and diestrus (van Goethem et al., 2012).

Estrogen treatment is reported to affect memory, especially hippocampus-dependent memory, and this hormone can enhance LTP (Foy, Henderson, Berger, \& Thompson, 2000). Many studies have reported that female sex steroid hormones can be a protective agent for a range of neural insults, experimental brain injuries, as well as neurodegenerative diseases (Hogervorst, Williams, Budge, Riedel, \& Jolles, 2000; Pike, Carroll, Rosario, \& Barron, 2009; Sherwin \& Henry, 2008), though the hidden mechanisms underlying this matter is not fully understood (Sherwin \& Henry, 2008). In addition, aging female rats with lower estrogen plasma levels in the estropause phase are known to perform worse in a Morris water maze compared to female rats with higher estrogen levels (Hajali et al., 2012). In general, estrogen has been effective in cognitive functions that are complex and time-dependent, including learning and memory processes and these effects can be seen in both animals and humans (Gasbarri et al., 2012). The basal forebrain cholinergic system and hippocampus are the brain systems that may play a role in both attention and memory effects of estrogens (Gibbs \& Aggarwal, 1998).

The male rats that experienced SD exhibited significant impairments in learning and memory paradigms such as radial arm water maze (Alhaider, Aleisa, Tran, Alzoubi, \& Alkadhi, 2010; Zagaar et al., 2012), MWM (Guan, Peng, \& Fang, 2004; Hajali et al., 2012), plus maze discriminative avoidance task (Alvarenga et al., 2008), passive and active avoidance task (Coll-Andreu, Marti-Nicolovius, \& Morgado-Bernal, 1991; van Hulzen \& Coenen, 1982) and novel object recognition test (Chen, Tian, \& Ke, 2014). Furthermore, SD reduces capability for the retention of new information, and disrup- 
tions in memory consolidation can be seen (Ravassard et al., 2009; Vecsey et al., 2009). These reports are consistent with our results. Our results in the novel object recognition test showed that $\mathrm{SD}$ impaired the ability of rats in distinguishing novel objects and other studies also showed this impairment (Binder et al., 2012; Palchykova et al., 2006). Voluntary exercise in our study could improve this impairment. Other studies confirmed our results (García-Capdevila, Portell-Cortés, Torras-Garcia, Coll-Andreu, \& Costa-Miserachs, 2009) but we did not observe any significant effect of only voluntary exercise on novel object recognition. However, some studies have reported the effect of voluntary exercise alone. It seems that the different outcome is due to the duration of exercise (Nichol, Deeny, Seif, Camaclang, \& Cotman, 2009; Yuede et al., 2009), novel object recognition test protocol, age, and gender (Hopkins \& Bucci, 2010; Hopkins, Nitecki, \& Bucci, 2011).

It is believed that deleterious alterations in intracellular signaling molecules, as well as receptors, including NMDA (Chen, Hardy, Zhang, LaHoste, \& Bazan, 2006) and AMPA receptors (Hagewoud et al., 2010), lead to the negative effects of SD on learning and memory (Zagaar et al., 2012). Our data demonstrated that SD increased anxiety-like behaviors in the open field test. Other studies revealed that SD could lead to anxiety-like behaviors in an elevated plus maze (Silva, et al., 2004) and open field test (Vollert et al., 2011). Animals that experienced voluntary physical exercise before SD could improve anxiety-like behaviors compared to SD group. Similar studies had demonstrated that forced exercise ameliorated anxiety-like behaviors in sleep-deprived rats (Vollert et al., 2011). Several studies have acquired controversial results by analyzing the relationship between sleep deprivation and anxiety in animal models. Anxiogenic effects were reported in some literature (Vollert et al., 2011) whereas some showed that SD did not exhibit elevated corticosterone level and no change was seen in anxious behavior (Hajali et al., 2012). Furthermore, regular treadmill exercise leads to serum corticosterone level normalization and reduction in anxious behaviors induced by SD (Vollert et al., 2011). Our findings also demonstrated that voluntary exercise prior to SD could reduce anxiety-like behaviors but both of SD and voluntary exercise in this study did not make any significant changes in corticosterone level.

Our data also revealed that SD and voluntary exercise did not exhibit a significant effect on voluntary locomotion in the open field test, which is consistent with other investigations (Hajali et al., 2012).
Based on our findings, removing the ovaries enhances the sensitivity of female rats to the deleterious effects of sleep deprivation on spatial learning compared to the intact female rats. Consistently, in another study, sleep-deprived-OVX female rats exhibited significantly more impairment in learning in the spatial learning task of the MWM test compared to sleep deprived-intact female animals (Hajali et al., 2012). It has been also well documented that female sex steroid hormones can have protective effects against a wide range of neural insults, experimental brain injuries, and neurodegenerative diseases (Hogervorst et al., 2000; Pike, Carroll, Rosario, \& Barron, 2009; Scharfman \& Maclusky, 2005).

Though the hidden mechanism underlying this process is not well understood (Sherwin \& Henry, 2008), some human studies have reported that postmenopausal women are more vulnerable to the harmful effects of poor sleep on cognitive performances (Manber \& Armitage, 1999; Paul, Turek, \& Kryger, 2008). Our results have revealed that voluntary exercise can have beneficial effects on long-term memory in sleep-deprived OVX female rats. We also showed that voluntary exercise can completely ameliorate sleep deprivation memory decline because memory performance in the SD-exercise groups had reached a similar level compared to the control groups. Other studies proved that voluntary running can induce neurotrophic factors such as Brain-Derived Neurotrophic Factor (BDNF) and fibroblast growth factor in CNS (O'Dell et al., 2007). Voluntary exercise leads to a higher rate of cell proliferation as well as neurogenesis in different locations such as the dentate gyrus in the hippocampus and it also improves learning and memory (Van Praag, Kempermann, \& Gage, 1999). The adolescent male and female hippocampus are differentially sensitive to the potentiating effect of voluntary exercise on synaptic plasticity (Titterness, Wiebe, Kwasnica, Keyes, \& Christie, 2011). Of course, we neither investigated the role of signaling molecules nor carried out electrophysiological studies in the present study. It seems that further tests are required to examine the effect of voluntary physical exercise on the expression of BDNF mRNA and other signaling molecules and electrophysiological properties in OVX female rats following $72 \mathrm{~h} \mathrm{SD}$.

\section{Conclusion}

In conclusion, the findings of this study revealed that voluntary wheel running exercise can improve SD-induced impairments in spatial learning and memory and recognition memory in the OVX female rats. Also, voluntary exercise could ameliorate anxiety-like behaviors in sleep-deprived OVX female rats although our findings revealed that sleep deprivation and voluntary exercise 
did not have a significant effect on locomotor activity in OVX female rats.

\section{Ethical Considerations}

\section{Compliance with ethical guidelines}

There were no ethical considerations to be considered in this research.

\section{Funding}

This study was funded by a grant (Grant No.958892) from the National Institute for Medical Research Development (NIMAD).

\section{Authors' contributions}

Conceptualization and supervision: Vahid Sheibani and Khadijeh Esmaeilpour; Methodology, investigation, and Writing - review \& editing: All authors.

\section{Conflict of interest}

The authors declared no conflict of interest.

Acknowledgments

We would like to thank the NIMAD and Kerman Neuroscience Research Center.

\section{References}

Aguiar Jr, A. S., Castro, A. A., Moreira, E. L., Glaser, V., Santos, A. R., \& Tasca, C. I., et al. (2011). Short bouts of mild-intensity physical exercise improve spatial learning and memory in aging rats: involvement of hippocampal plasticity via AKT, CREB and BDNF signaling. Mechanisms of Ageing and Development, 132(11-12), 560-7. [DOI:10.1016/j.mad.2011.09.005] [PMID]

Alhaider, I. A., Aleisa, A. M., Tran, T. T., Alzoubi, K. H., \& Alkadhi, K. A. (2010). Chronic caffeine treatment prevents sleep deprivation-induced impairment of cognitive function and synaptic plasticity. Sleep, 33(4), 437-44. [DOI:10.1093/ sleep/33.4.437] [PMID] [PMCID]

Alvarenga, T. A., Patti, C. L., Andersen, M. L., Silva, R. H., Calzavara, M. B., \& Lopez, G. B., et al. (2008). Paradoxical sleep deprivation impairs acquisition, consolidation, and retrieval of a discriminative avoidance task in rats. Neurobi- ology of Learning and Memory, 90(4), 624-32. [DOI:10.1016/j. nlm.2008.07.013] [PMID]

Ben, J., Soares, F. M., Scherer, E. B., Cechetti, F., Netto, C. A., \& Wyse, A. T. (2010). Running exercise effects on spatial and avoidance tasks in ovariectomized rats. Neurobiology of Learning and Memory, 94(3), 312-7. [DOI:10.1016/j.nlm.2010.07.003] [PMID]

Binder, S., Baier, P. C., Mölle, M., Inostroza, M., Born, J., \& Marshall, L. (2012). Sleep enhances memory consolidation in the hippocampus-dependent object-place recognition task in rats. Neurobiology of Learning and Memory, 97(2), 213-9. [DOI:10.1016/j.nlm.2011.12.004] [PMID]

Brinton, R. D. (2009). Estrogen-induced plasticity from cells to circuits: Predictions for cognitive function. Trends in Pharmacological Sciences, 30(4), 212-22. [DOI:10.1016/j. tips.2008.12.006] [PMID] [PMCID]

Burghardt, P. R., Fulk, L. J., Hand, G. A., \& Wilson, M. A. (2004) The effects of chronic treadmill and wheel running on behavior in rats. Brain Research, 1019(1), 84-96. [DOI:10.1016/j. brainres.2004.05.086] [PMID]

Chen, C., Hardy, M., Zhang, J., LaHoste, G. J., \& Bazan, N. G. (2006). Altered NMDA receptor trafficking contributes to sleep deprivation-induced hippocampal synaptic and cognitive impairments. Biochemical and Biophysical Research Communications, 340(2), 435-40. [DOI:10.1016/j.bbrc.2005.12.021] [PMID]

Chen, L., Tian, S., \& Ke, J. (2014). Rapid eye movement sleep deprivation disrupts consolidation but not reconsolidation of novel object recognition memory in rats. Neuroscience Letters, 563, 12-6. [DOI:10.1016/j.neulet.2014.01.024] [PMID]

Coll-Andreu, M., Marti-Nicolovius, M., \& Morgado-Bernal, I (1991). Facilitation of shuttle-box avoidance by the platform method: temporal effects. Physiology \& Behavior, 49(6), 1211-5. [DOI:10.1016/0031-9384(91)90353-P]

Cotman, C. W., \& Berchtold, N. C. (2002). Exercise: A behavioral intervention to enhance brain health and plasticity. Trends in Neurosciences, 25(6), 295-301. [DOI:10.1016/S01662236(02)02143-4]

Diekelmann, S., \& Born, J. (2010). The memory function of sleep. Nature Reviews Neuroscience, 11(2), 114-26. [DOI:10.1038/ nrn2762] [PMID]

Dishman, R. K. (1997). Brain monoamines, exercise, and behavioral stress: Animal models. Medicine \& Science in Sports \& Ex ercise, 29(1), 63-74. [DOI:10.1097/00005768-199701000-00010] [PMID]

Dzaja, A., Arber, S., Hislop, J., Kerkhofs, M., Kopp, C., Pollmächer, T., \& et al. (2005). Women's sleep in health and disease. Journal of Psychiatric Research, 39(1), 55-76. [DOI:10.1016/j. jpsychires.2004.05.008] [PMID]

Esmaeilpour, K., Sheibani, V., Shabani, M., \& Mirnajafi-Zadeh, J. (2017). Effect of low frequency electrical stimulation on seizure-induced short- and long-term impairments in learning and memory in rats. Physiology $\mathcal{E}$ Behavior, 168, 112-21. [DOI:10.1016/j.physbeh.2016.11.001] [PMID]

Forest, G., \& Godbout, R. (2000). Effects of sleep deprivation on performance and EEG spectral analysis in young adults. Brain and Cognition, 43(1-3), 195-200. https://psycnet.apa. org/record/2000-15775-039 
Foy, M. R., Baudry, M., Akopian, G. K., \& Thompson, R. F. (2010). Regulation of hippocampal synaptic plasticity by estrogen and progesterone. In Vitamins \& Hormones, 82, 219-39. [DOI:10.1016/S0083-6729(10)82012-6]

Foy, M. R., Henderson, V. W., Berger, T. W., \& Thompson, R. F. (2000). Estrogen and neural plasticity. Current Directions in Psychological Science, 9(5), 148-52. [DOI:10.1111/14678721.00081]

García-Capdevila, S., Portell-Cortés, I., Torras-Garcia, M., CollAndreu, M., \& Costa-Miserachs, D. (2009). Effects of longterm voluntary exercise on learning and memory processes: dependency of the task and level of exercise. Behavioural Brain Research, 202(2), 162-70. [DOI:10.1016/j.bbr.2009.03.020] [PMID]

Gasbarri, A., Tavares, M. C. H., Rodrigues, R. C., Tomaz, C., \& Pompili, A. (2012). Estrogen, cognitive functions and emotion: an overview on humans, non-human primates and rodents in reproductive years. Reviews in the Neurosciences, 23(56), 587-606. [DOI:10.1515/revneuro-2012-0051]

Gibbs, R. B., \& Aggarwal, P. (1998). Estrogen and basal forebrain cholinergic neurons: Implications for brain aging and Alzheimer's disease-related cognitive decline. Hormones and Behavior, 34(2), 98-111. [DOI:10.1006/hbeh.1998.1451] [PMID]

Guan, Z., Peng, X., \& Fang, J. (2004). Sleep deprivation impairs spatial memory and decreases extracellular signal-regulated kinase phosphorylation in the hippocampus. Brain Research, 1018(1), 38-47. [DOI:10.1016/j.brainres.2004.05.032] [PMID]

Hagewoud, R., Havekes, R., Novati, A., Keijser, J. N., Van der Zee, E. A., \& Meerlo, P. (2010). Sleep deprivation impairs spatial working memory and reduces hippocampal AMPA receptor phosphorylation. Journal of Sleep Research, 19(2), 280-8. [DOI:10.1111/j.1365-2869.2009.00799.x] [PMID]

Hajali, V., Sheibani, V., Esmaeili-Mahani, S., \& Shabani, M. (2012). Female rats are more susceptible to the deleterious effects of paradoxical sleep deprivation on cognitive performance. Behavioural Brain Research 228(2), 311-18. [DOI:10.1016/j.bbr.2011.12.008] [PMID]

Henderson, V. W., \& Sherwin, B. B. (2007). Surgical versus natural menopause: cognitive issues. Menopause, 14(3 Pt 2), 572-79. [DOI:10.1097/gme.0b013e31803df49c] [PMID]

Hogervorst, E., Williams, J., Budge, M., Riedel, W., \& Jolles, J. (2000). The nature of the effect of female gonadal hormone replacement therapy on cognitive function in post-menopausal women: A meta-analysis. Neuroscience, 101(3), 485512. [DOI:10.1016/S0306-4522(00)00410-3]

Hopkins, M. E., \& Bucci, D. J. (2010). BDNF expression in perirhinal cortex is associated with exercise-induced improvement in object recognition memory. Neurobiology of Learning and Memory, 94(2), 278-284. [DOI:10.1016/j.nlm.2010.06.006] [PMID] [PMCID]

Hopkins, M. E., Nitecki, R., \& Bucci, D. J. (2011). Physical exercise during adolescence versus adulthood: differential effects on object recognition memory and brain-derived neurotrophic factor levels. Neuroscience, 194, 84-94. [DOI:10.1016/j.neuroscience.2011.07.071] [PMID] [PMCID]

Hoveida, R., Alaei, H., Oryan, S., Parivar, K., \& Reisi, P. (2011). Treadmill running improves spatial memory in an animal model of Alzheimer's disease. Behavioural Brain Research 216(1), 270-4. [DOI:10.1016/j.bbr.2010.08.003] [PMID]

Landers, M. R., Kinney, J. W., Allen, D. N., \& van Breukelen, F. (2013). A comparison of voluntary and forced exercise in protecting against behavioral asymmetry in a juvenile hemiparkinsonian rat model. Behavioural Brain Research, 248, 12128. [DOI:10.1016/j.bbr.2013.04.002] [PMID]

Leasure, J., \& Jones, M. (2008). Forced and voluntary exercise differentially affect brain and behavior. Neuroscience, 156(3), 456-65. [DOI:10.1016/j.neuroscience.2008.07.041] [PMID]

Lee, A. K., \& Wilson, M. A. (2002). Memory of sequential experience in the hippocampus during slow wave sleep. Neuron, 36(6), 1183-94. [DOI:10.1016/S0896-6273(02)01096-6]

Lin, T. W., Chen, S. J., Huang, T. Y., Chang, C. Y., Chuang, J. I., $\& \mathrm{Wu}, \mathrm{F}$. S., et al. (2012). Different types of exercise induce differential effects on neuronal adaptations and memory performance. Neurobiology of Learning and Memory, 97(1), 140-7. [DOI: 10.1016/j.nlm.2011.10.006] [PMID]

Liu, Y. F., Chen, H. I., Wu, C. L., Kuo, Y. M., Yu, L., \& Huang, A M., et al. (2009). Differential effects of treadmill running and wheel running on spatial or aversive learning and memory: roles of amygdalar brain-derived neurotrophic factor and synaptotagmin I. The Journal of Physiology, 587(Pt 13), 322131. [DOI:10.1113/jphysiol.2009.173088] [PMID] [PMCID]

Machado, R. B., Hipolide, D. C., Benedito-Silva, A. A., \& Tufik, S. (2004). Sleep deprivation induced by the modified multiple platform technique: Quantification of sleep loss and recovery. Brain Research, 1004(1-2), 45-51. [DOI:10.1016/j. brainres.2004.01.019] [PMID]

Manber, R., \& Armitage, R. (1999). Sex, steroids, and sleep: A review. Sleep, 22(5), 540-541. https://doi.org/10.1093/ sleep/22.5.540

Marlatt, M. W., Potter, M. C., Lucassen, P. J., \& van Praag, H. (2012). Running throughout middle-age improves memory function, hippocampal neurogenesis, and BDNF levels in female C57BL/6J mice. Developmental Neurobiology, 72(6), 943 52. [DOI:10.1002/dneu.22009] [PMID] [PMCID]

Mitchell, E. S., \& Woods, N. F. (2011). Cognitive symptoms during the menopausal transition and early postmenopause. Climacteric, 14(2), 252-61. [DOI:10.3109/13697137.20 10.516848] [PMID]

Nichol, K., Deeny, S. P., Seif, J., Camaclang, K., \& Cotman, C. W. (2009). Exercise improves cognition and hippocampal plasticity in APOE $\varepsilon 4$ mice. Alzheimer's \& Dementia, 5(4), 287 94. [DOI:10.1016/j.jalz.2009.02.006] [PMID] [PMCID]

O'Dell, S. J., Gross, N. B., Fricks, A. N., Casiano, B. D., Nguyen, T. B., \& Marshall, J. F. (2007). Running wheel exercise enhances recovery from nigrostriatal dopamine injury without inducing neuroprotection. Neuroscience, 144(3), 1141-51. [DOI:10.1016/j.neuroscience.2006.10.042] [PMID]

Palchykova, S., Winsky-Sommerer, R., Meerlo, P., Dürr, R., \& Tobler, I. (2006). Sleep deprivation impairs object recognition in mice. Neurobiology of Learning and Memory, 85(3), 26371. [DOI:10.1016/j.nlm.2005.11.005] [PMID]

Paul, K. N., Turek, F. W., \& Kryger, M. H. (2008). Influence of sex on sleep regulatory mechanisms. Journal of Women's Health, 17(7), 1201-08. [DOI:10.1089/jwh.2008.0841] [PMID] 
Pike, C. J., Carroll, J. C., Rosario, E. R., \& Barron, A. M. (2009). Protective actions of sex steroid hormones in Alzheimer's disease. Frontiers in Neuroendocrinology, 30(2), 239-58. [DOI:10.1016/j.yfrne.2009.04.015] [PMID] [PMCID]

Ploughman, M., Granter-Button, S., Chernenko, G., Tucker, B., Mearow, K., \& Corbett, D. (2005). Endurance exercise regimens induce differential effects on brain-derived neurotrophic factor, synapsin-I and insulin-like growth factor I after focal ischemia. Neuroscience, 136(4), 991-1001. [DOI:10.1016/j.neuroscience.2005.08.037] [PMID]

Rajizadeh, M. A., Esmaeilpour, K., Masoumi-Ardakani, Y., Bejeshk, M. A., Shabani, M., \& Nakhaee, N., et al. (2018). Voluntary exercise impact on cognitive impairments in sleep-deprived intact female rats. Physiology E Behavior, 188, 58-66. [DOI:10.1016/j.physbeh.2017.12.030] [PMID]

Rajizadeh, M. A., Sheibani, V., Bejeshk, M. A., Mohtashami Borzadaran, F., Saghari, H., \& Esmaeilpour, K. (2019). The effects of high intensity exercise on learning and memory impairments followed by combination of sleep deprivation and demyelination induced by etidium bromide. International Journal of Neuroscience, 129(12), 1166-78. [DOI:10.1080/ 00207454.2019.1640695] [PMID]

Ravassard, P., Pachoud, B., Comte, J. C., Mejia-Perez, C., ScoteBlachon, C., \& Gay, N., et al. (2009). Paradoxical (REM) sleep deprivation causes a large and rapidly reversible decrease in long-term potentiation, synaptic transmission, glutamate receptor protein levels, and ERK/MAPK activation in the dorsal hippocampus. Sleep, 32(2), 227-40. [DOI:10.1093/ sleep/32.2.227] [PMID] [PMCID]

Saadati, H., Esmaeili-Mahani, S., Esmaeilpour, K., Nazeri, M., Mazhari, S., \& Sheibani, V. (2015). Exercise improves learning and memory impairments in sleep deprived female rats. Physiology \& Behavior, 138, 285-91. [DOI:10.1016/j.physbeh.2014.10.006] [PMID]

Salari, M., Sheibani, V., Saadati, H., Pourrahimi, A., Esmaeelpour, K., \& Khodamoradi, M. (2015). The compensatory effect of regular exercise on long-term memory impairment in sleep deprived female rats. Behavioural Processes, 119, 50-57. [DOI:10.1016/j.beproc.2015.06.014] [PMID]

Scharfman, H. E., \& Maclusky, N. J. (2005). Similarities between actions of estrogen and BDNF in the hippocampus: coincidence or clue? Trends Neurosci, 28(2), 79-85. [DOI:10.1016/j. tins.2004.12.005] [PMID]

Shangold, M. M. (1990). Exercise in the menopausal woman. Obstetrics \& Gynecology, 75(4 Suppl), 53S-58S. [DOI:10.1097/00006250-199004001-00012]

Sheibani, V., Rafie, F., Shahbazi, M., Naghdi, N., \& Sheikh, M. (2017). Comparison of voluntary and forced exercise effects on motor behavior in 6-hydroxydopamine-lesion rat model of Parkinson's disease. Sport Sciences for Health, 13(1), 203-11. [DOI:10.1007/s11332-017-0354-9]

Sherwin, B. B., \& Henry, J. F. (2008). Brain aging modulates the neuroprotective effects of estrogen on selective aspects of cognition in women: A critical review. Frontiers in Neuroendocrinology, 29(1), 88-113. [DOI:10.1016/j.yfrne.2007.08.002] [PMID]

Silva, R. H., Chehin, A. B., Kameda, S. R., Takatsu-Coleman, A. L., Abilio, V. C., \& Tufik, S., et al. (2004). Effects of pre- or posttraining paradoxical sleep deprivation on two animal models of learning and memory in mice. Neurobiology of Learning and Memory, 82(2), 90-8. [DOI:10.1016/j.nlm.2004.04.005] [PMID]

Silva, R. H., Kameda, S. R., Carvalho, R. C., Takatsu-Coleman, A. L., Niigaki, S. T., \& Abilio, V. C., et al. (2004). Anxiogenic effect of sleep deprivation in the elevated plus-maze test in mice. Psychopharmacology (Berl), 176(2), 115-22. [DOI:10.1007/ s00213-004-1873-z] [PMID]

Suchecki, D., \& Tufik, S. (2000). Social stability attenuates the stress in the modified multiple platform method for paradoxical sleep deprivation in the rat. Physiology \& Behavior, 68(3), 309-16. [DOI:10.1016/S0031-9384(99)00181-X]

Titterness, A., Wiebe, E., Kwasnica, A., Keyes, G., \& Christie, B (2011). Voluntary exercise does not enhance long-term potentiation in the adolescent female dentate gyrus. Neuroscience, 183, 25-31. [DOI:10.1016/j.neuroscience.2011.03.050] [PMID]

van Goethem, N. P., Rutten, K., van der Staay, F. J., Jans, L. A., Akkerman, S., \& Steinbusch, H. W., et al. (2012). Object recognition testing: rodent species, strains, housing conditions, and estrous cycle. Behavioural Brain Research, 232(2), 323-334. [DOI:10.1016/j.bbr.2012.03.023] [PMID]

van Hulzen, Z. J., \& Coenen, A. M. (1982). Effects of paradoxical sleep deprivation on two-way avoidance acquisition. Physiology \& Behavior, 29(4), 581-7. [DOI:10.1016/00319384(82)90223-2]

Van Praag, H., Kempermann, G., \& Gage, F. H. (1999). Running increases cell proliferation and neurogenesis in the adult mouse dentate gyrus. Nature Neuroscience, 2(3), 266270. [DOI:10.1038/6368] [PMID]

Vaziri, Z., Abbassian, H., Sheibani, V., Haghani, M., Nazeri, M., Aghaei, I., \& Shabani, M. (2015). The therapeutic potential of Berberine chloride hydrate against harmaline-induced motor impairments in a rat model of tremor. Neuroscience Letters, 590, 84-90. [DOI:10.1016/j.neulet.2015.01.078] [PMID]

Vecsey, C. G., Baillie, G. S., Jaganath, D., Havekes, R., Daniels, A., \& Wimmer, M., et al. (2009). Sleep deprivation impairs cAMP signalling in the hippocampus. Nature, 461(7267), 1122-25. [DOI:10.1038/nature08488] [PMID] [PMCID]

Vollert, C., Zagaar, M., Hovatta, I., Taneja, M., Vu, A., \& Dao, A., et al. (2011). Exercise prevents sleep deprivation-associated anxiety-like behavior in rats: Potential role of oxidative stress mechanisms. Behavioural Brain Research, 224(2), 23340. [DOI:10.1016/j.bbr.2011.05.010] [PMID]

Walker, M. P., \& Stickgold, R. (2004). Sleep-dependent learning and memory consolidation. Neuron, 44(1), 121-33. [DOI:10.1016/j.neuron.2004.08.031] [PMID]

Yoo, S. S., Hu, P. T., Gujar, N., Jolesz, F. A., \& Walker, M. P. (2007). A deficit in the ability to form new human memories without sleep. Nature Neuroscience, 10(3), 385-92. [DOI:10.1038/nn1851] [PMID]

Yuede, C. M., Zimmerman, S. D., Dong, H., Kling, M. J., Bero, A. W., \& Holtzman, D. M., et al. (2009). Effects of voluntary and forced exercise on plaque deposition, hippocampal volume, and behavior in the Tg2576 mouse model of Alzheimer's disease. Neurobiology of Disease, 35(3), 426-32. [DOI:10.1016/j.nbd.2009.06.002] [PMID] [PMCID]

Zagaar, M., Alhaider, I., Dao, A., Levine, A., Alkarawi, A., Alzubaidy, M., \& Alkadhi, K. (2012). The beneficial effects of regular exercise on cognition in REM sleep deprivation 
Behavioral, electrophysiological and molecular evidence.

Neurobiology of Disease, 45(3), 1153-1162. [DOI:10.1016/j.

nbd.2011.12.039] [PMID] 


\title{
Research Paper: Effects of Pretreatment With Ginseng Extract on Dopamine D2 Receptor Analgesia
}

\author{
Mahnaz Taherianfard $^{1 *}$ (1), Somaye Aalami ${ }^{1}$
}

1. Department of Basic Sciences, School of Veterinary Medicine, Shiraz University, Shiraz, Iran.

\begin{tabular}{l|l|}
$\begin{array}{l}\text { Use vour device to san } \\
\text { and read the artice online }\end{array}$ & $\begin{array}{l}\text { Cftation: Taherianfard, M., \& Aalami, S. (2020). Effects of Pretreatment With Ginseng Extract on Dopamine D2 Receptor } \\
\text { Analgesia. Basic and Clinical Neuroscience, 11(5), 587-594. http://dx.doi.org/10.32598/bcn.9.10.425 }\end{array}$ \\
dof http://dx.doi.org/10.32598/bcn.9.10.425
\end{tabular}

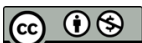

Article info:

Received: 11 Jul 2019

First Revision: 10 Aug 2019

Accepted: 19 Dec 2019

Available Online: 01 Sep 2020

Keywords:

Ginseng, D2 agonist, D2 antagonist, Formalin test

\section{ABSTRACT}

Introduction: The ginseng extract is an herb that has been used for many purposes such as analgesic effect. Dopamine D2 receptors are involved in the regulation of pain in humans. Therefore, the present investigation aims to study how pretreatment with aqueous-alcoholic extract of ginseng can affect dopamine D2 receptors' pain sensitivity.

Methods: We used 45 adult male rats weighing $250 \pm 20$ for this study. Animals were maintained in a standard condition at a temperature of $21{ }^{\circ} \mathrm{C}-24^{\circ} \mathrm{C}$. The experimental groups were as follows: 1. Sham 1 (intraperitoneal [IP] injection of normal saline); 2. Sham 2 (intracerebroventricular [ICV] injection of artificial cerebrospinal fluid [ACSF]); 3. Experimental 1 (IP injection of ginseng extract); 4 and 5. Experimental groups 2 and 3 (IP injection of ginseng extract + bromocriptine 10 and $30 \mu \mathrm{g} /$ rat by ICV injection); 6 and 7) experimental groups 4 and 5 (IP injection of ginseng extract + chlorpromazine 20 and $40 \mu \mathrm{g}$ ) rat by ICV injection). Ginseng extract $100 \mathrm{mg} / \mathrm{kg} / \mathrm{d}$ was used for 7 days. Pain sensitivity test was done in all groups with the formalin test. Lateral ventricles of the rats were cannulated unilaterally by the stereotaxic procedure.

Results: Our data showed that ginseng $(100 \mathrm{mg} / \mathrm{kg} / \mathrm{d})$ significantly $(\mathrm{P}<0.05)$ decreased pain sensitivity compared to the sham 1 group. Bromocriptine in two doses significantly decreased pain sensitivity compared to the sham 2 group. Chlorpromazine in high doses significantly increased pain sensitivity compared to the sham 2 group.

Conclusion: The present results indicate that ginseng can modulate the D2 receptor of the dopamine system in the control of pain sensitivity in the formalin test. Because bromocriptine and ginseng have similar effects, it seems that they had synergistic effects. 


\section{Highlights}

- Ginseng and bromocriptine had an analgesic effect.

- An analgesic effect of ginseng and bromocriptine is synergistic but no additive.

- Hyperalgesic effect of chlorpromazine is more potent than the analgesic effect of ginseng.

\section{Plain Language Summary}

Pain is physical and mental suffering that may arise by internal or external stimuli. Dopamine is one of the main chemical substances of the brain. It seems that the dopamine has analgesic effect. The analgesic effect of dopamine is through dopaminergic D2 receptors. On the other hand, Ginseng is an herbal plant and the roots of this plant contain a class of steroid that is responsible for its pharmacological activity. Ginseng has analgesic, antioxidant, anti-inflammatory, anti-apoptotic, and anti-aging properties. Some investigator stated that ginseng not only cause the release of dopamine directly or indirectly through the cholinergic system, but also can directly affect dopamine D2 receptors. According to the present results, ginseng administration $(100 \mathrm{mg} / \mathrm{kg} /$ day $)$ for 7 days had an analgesic effect, and bromocriptine (D2 receptor agonist) administration in two different doses after pretreatment with ginseng (100 mg/ $\mathrm{kg}$ /day) had an analgesic effect the same as ginseng alone. Therefore, it seems that bromocriptine and ginseng had a synergistic but no additive effect. Finally, chlorpromazine (D2 receptor antagonist) administration in two different doses after pretreatment with ginseng $(100 \mathrm{mg} / \mathrm{kg} /$ day $)$ had hyperalgesic effects compared to ginseng alone. Therefore, the hyperalgesic effect of chlorpromazine is more potent than the analgesic effect of ginseng. According to our data ginseng and D2 receptor agonist had the same analgesic effect.

\section{Introduction}

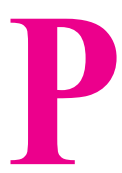

ain is physical and mental suffering that may arise by internal or external stimuli. Pain regulation is a complex process that depends on the interaction of many physiological, neurological, and hormonal factors. By changing the level of chemical mediators, some environmental events reduce or increase pain sensitivity. These chemical mediators are very effective in pain relief (Garland, 2012).

Dopamine is one of the main neurotransmitters of the central nervous system and many neurological and psychiatric diseases are related to its secretion and functions (Dauer \& Przedborski, 2003). It seems that the analgesic effect of the dopaminergic system is through the endogenous opioid receptor (Volkow, 2010). These effects on pain processing are mainly done by striatal dopaminergic D2 receptors (Becker et al., 2013). Besides, it is reported that activation of dopamine $\mathrm{D} 2$ receptor through descending endogenous pain-control pathways is essential (Dauer \& Przedborski, 2003).

Ginseng is an herbal plant belonging to the Panax genus of the family Araliaceae (Rhim, Kim, Lee, Oh, \& Nah, 2002). The roots of this plant contain a class of ste- roid glycosides called ginsenoside that is responsible for its pharmacological activity (Chang, Seo, Gyllenhaal, $\&$ Block, 2003). The scientific names of ginsenosides are triterpenoid saponins or sometimes panaxoside in ginseng root (Sun, 2004). Ginseng has antioxidant, anti-inflammatory, anti-apoptotic, and anti-aging properties. These effects include increased neuronal survival, growth, and development of neuron and prevention from neuronal death (Rausch, Liu, Gille, \& Radad, 2006). Kim, Shim, Chung, and Lee (2006) stated that ginseng saponins not only cause the release of dopamine directly or indirectly through the cholinergic system, but also can directly affect dopamine D2 receptors (Kim et al., 2006). On the other hand, ginsenosides have partial neurotrophic and neuroprotective effects on dopaminergic neurons in culture. Therefore, they increase cell survival by decreasing the release of lactate dehydrogenase and preventing mitochondrial membrane potential loss (Sandoval-Avila et al., 2018).

Despite many investigations on dopamine D2 receptor and pain and the relation between ginseng extract and pain, the simultaneous analgesic effect of these two factors has not been evaluated. Therefore, we aimed to investigate the effect of dopamine D2 receptor agonis (bromocriptine) and antagonist (chlorpromazine) on 


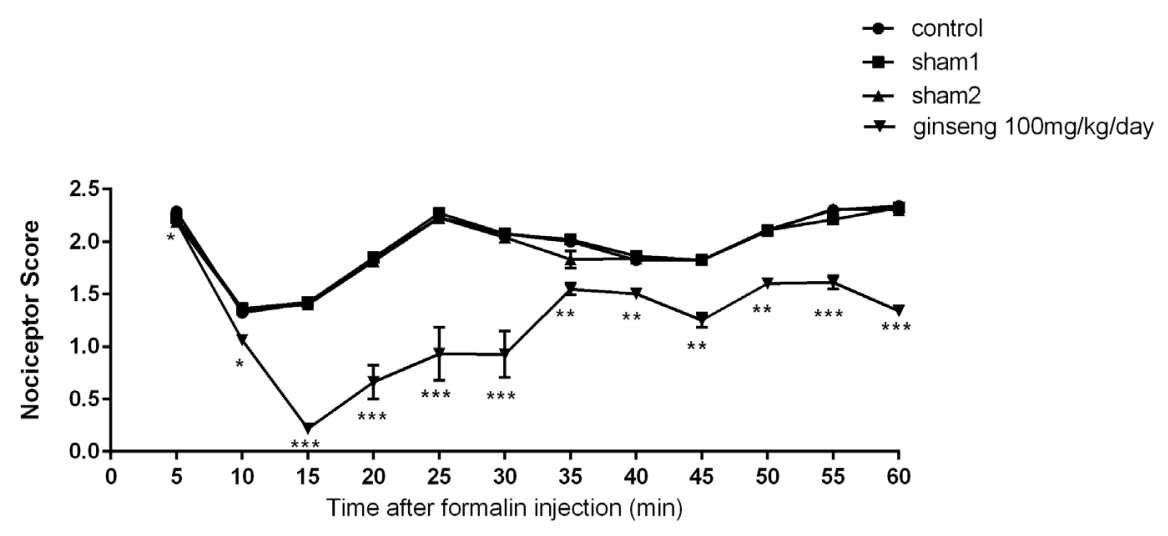

Figure 1. Effect of ginseng $(100 \mathrm{mg} / \mathrm{kg} / \mathrm{d})$ on nociceptive scores (Mean $\pm \mathrm{SE})$

NEUR SCIENCE

* Difference between ginger group in comparison to control, sham 1 and sham 2 groups; ${ }^{*} \mathrm{P}<0.05$, ** $\mathrm{P}<0.01$, *** $\mathrm{P}<0.001$.

pain sensitivity after ginseng extract administration $(100$ $\mathrm{mg} / \mathrm{kg} / \mathrm{d}$ ) for 7 days.

\section{Materials and Methods}

A total number of 56 adult male Wistar rats weighing approximately $250 \pm 20 \mathrm{~g}$ in standard conditions $(12 / 12$ hour light/dark cycle at $22 \pm 2^{\circ} \mathrm{C}$ ) were used. They had access to food and water ad libitum.

The rats were randomly divided into 7 groups $(n=8)$, as follows: 1 . Sham 1: healthy rats received IP injection of normal saline; sham 2 . Rats were unilaterally received Artificial Cerebrospinal Fluid (ACSF) by Intracerebroventricular (ICV) injection into the lateral ventricle; 3. experimental 1: rats received IP injection of ginseng extract; 4 and 5. Experimental 2 and 3: Rats received IP injection of ginseng extract + unilateral ICV injection of bromocriptine 10 or $30 \mu \mathrm{g} / \mathrm{rat} ; 6$ and 7. Experimental 4 and 5: rats received IP injection of ginseng extract + unilateral ICV injection of chlorpromazine 20 or $40 \mu \mathrm{g} / \mathrm{rat}$.

IP injections of normal saline and ginseng extract $(100 \mathrm{mg}$ $\mathrm{kg} / \mathrm{d}$ ) were done for 7 days. Formalin test was performed 30 min after IP injection of normal saline or ginseng extract in the sham 1 and experiment 1 groups and $30 \mathrm{~min}$ after ICV injection in groups that received drugs or ACSF. Hydro-alcoholic extract of ginseng was prepared according to Palaniyandi, Suh, and Yang (2017) study.

To evaluate the pain sensitivity, formalin solution $(50 \mu \mathrm{L})$ was subcutaneously injected into the dorsal surface of the animal's right hind paw ( $2.5 \%$ in normal saline). The pain score was recorded every 15 seconds for 60 minutes. The score would be 0 if the animal showed no reaction, 1 if the animal did not rely on the injected paw, 2 if the animal holds its paw up, and 3 if the rat licks and/or bites the injected paw. The results of all 15-s checks were averaged for every 5 minutes and considered for data analysis.

For the stereotaxic procedure, the animals were anesthetized with an IP injection of ketamine $10 \%(100 \mathrm{mg} / \mathrm{kg})$ and xylazine $2 \%(10 \mathrm{mg} / \mathrm{kg})$. The rats were fixed in the stereotaxic apparatus using blunt ear bars. The skull was carefully exposed and stainless steel guide cannula (23-gauge needle) was inserted unilaterally into the lateral ventricle. The coordinates for lateral ventricle according to bregma were $0.5 \mathrm{~mm}$ anterior and $1.5 \mathrm{~mm}$ lateral to the midline and $3.5 \mathrm{~mm}$ below to cortex. The guide cannula via dental acrylic cement and two tiny stainless steel screws were fixed to the skull. In the end, the animals were given 7 days recovery period.

\subsection{Statistical analysis}

For data analysis, we used SPSS V. 21. The data were analyzed by One-way ANOVA, repeated measure ANOVA, and the Tuckey post-hoc test. P-values less than 0.05 were considered significant.

\section{Results}

According to ANOVA, IP injection of ginseng $100 \mathrm{mg} /$ $\mathrm{kg} / \mathrm{d}$ for 7 days significantly reduced pain sensitivity in the early phase $\left(\mathrm{F}_{9}=61.2, \mathrm{P}=0.0\right)$, intermediate phase $\left(\mathrm{F}_{9}=16.8\right.$, $\mathrm{P}=0.0)$, and late phase $\left(\mathrm{F}_{9}=92.8, \mathrm{P}=0.0\right)$ of formalin test compared to the control and sham1 groups (Figure 1).

One-way ANOVA results indicate that ICV injection of bromocriptine $10 \mu \mathrm{g} / \mathrm{rat}$ after IP injection of ginseng 100 $\mathrm{mg} / \mathrm{kg} / \mathrm{d}$ for 7 days significantly $(\mathrm{P}<0.0001)$ reduced pain sensitivity during 60 minutes of formalin test compared to the control and sham 1 groups. The ICV injection of bromocriptine $30 \mu \mathrm{g} / \mathrm{rat}$ after IP injection of ginseng (100 $\mathrm{mg} / \mathrm{kg} / \mathrm{d}$ ) for 7 days had similar effects except that in the 


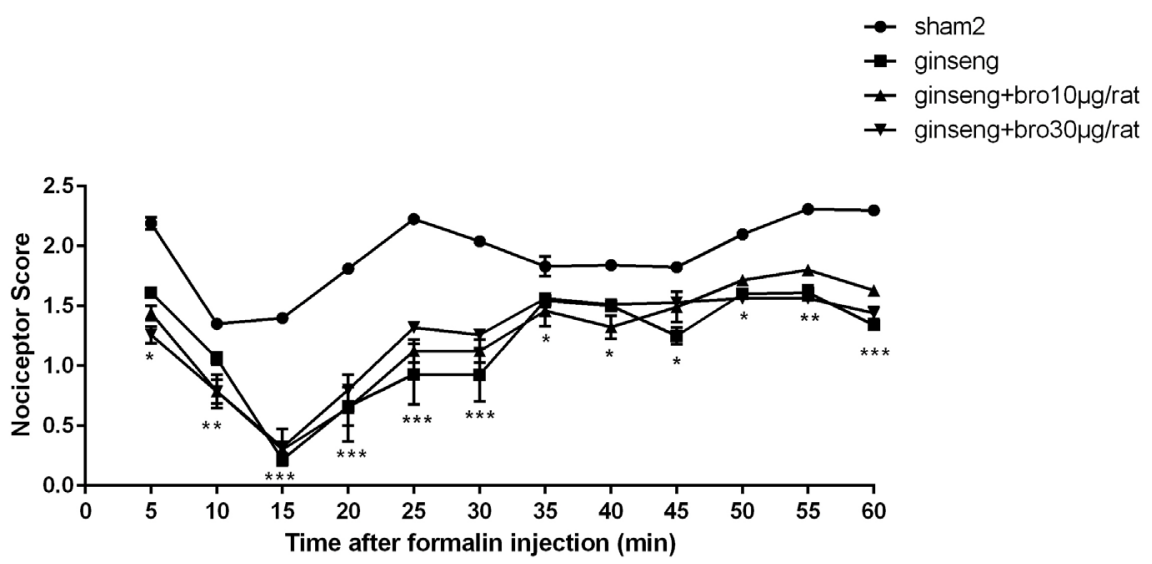

NEUR SCIENCE

Figure 2. Effect of bro (bromocriptine) 10 and $30 \mu \mathrm{g} /$ rat on nociceptive scores (Mean $\pm \mathrm{SE}$ ) in rats pretreated by ginseng (100 $\mathrm{mg} / \mathrm{kg} / \mathrm{d}$ ) for 7 days

* Significant difference between bro 10 and $30 \mu \mathrm{g} /$ rat group in comparison to sham 2 group; ${ }^{\mathrm{P}}<0.05$, ${ }^{* *} \mathrm{P}<0.01$, ${ }^{* * *} \mathrm{P}<0.001$.

25 th and 45 th minutes there was no difference compared to the control and sham 2 groups. Bromocriptine in two doses significantly decrease pain sensitivity in the early phase $\left(\mathrm{F}_{9}=61.2, \mathrm{P}=0.0\right)$, intermediate phase $\left(\mathrm{F}_{9}=16.8\right.$, $\mathrm{P}=0.0)$, and late phase $\left(\mathrm{F}_{9}=92.8, \mathrm{P}=0.0\right)$ of formalin test compared to the control and sham 2 groups but does not have any difference with ginseng group (Figure 2).

Chlorpromazine $(20 \mu \mathrm{g} / \mathrm{rat})$ had no significant effect on pain sensitivity compared to the control and sham 2 groups; while it significantly increased pain sensitivity in the early phase $\left(\mathrm{F}_{9}=61.2, \mathrm{P}=0.0\right)$, intermediate phase $\left(\mathrm{F}_{9}=16.8, \mathrm{P}=0.0\right)$, and late phase $\left(\mathrm{F}_{9}=92.8, \mathrm{P}=0.0\right)$ of formalin test compared with the ginseng group (Figure 3 ). Chlorpromazine $(40 \mu \mathrm{g} / \mathrm{rat})$ significantly increased pain sensitivity in the early phase $(\mathrm{F}=61.2, \mathrm{P}=0.0)$, intermediate phase $\left(\mathrm{F}_{9}=16.8, \mathrm{P}=0.0\right)$ and late phase $\left(\mathrm{F}_{9}=92.8, \mathrm{P}=0.0\right)$ of formalin test compared to the control, sham 2 , and ginseng groups (Figure 3).

\section{Discussion}

The present study demonstrates that the hydro-alcoholic extract of ginseng $100 \mathrm{mg} / \mathrm{kg} / \mathrm{d}$ has an analgesic effect in phase 1 and phase 2 of the formalin test. Previous studies revealed that ginseng saponins have analgesic effects in the writhing test and phase 2 of the formalin test (Nabata, Saito, \& Takagi, 1973; Shin et al., 1997).

The mechanism of the analgesic action of ginseng by formalin test has not been specified, yet. Nevertheless, it is likely that saponins of ginseng attach to the nonopioid receptors at the cell surface and regulate the voltage-gated calcium channels. In other words, the calcium channels are inhibited by ginseng saponins. Volt- age-gated calcium channels play a key role in releasing pain neurotransmitters in presynaptic nerve endings of efferent neurons and its blocking decreases pain to some extent (Nah \& McCleskey, 1994).

The other analgesic mechanism of ginseng in the formalin test is that ginseng saponins or ginsenosides probably affect the dopaminergic activity of the central nervous system (Jun, Bae, Kim, Koo, \& Kim, 2015). Besides the postsynaptic effect of ginseng on voltage-gated channels, it may also affect the presynaptic signal pathway in the dopamine system and thereby increasing dopamine release from nucleus accumbens, as a result, causes pain relief in the formalin test (Mancusoa \& Santangelob, 2017; Nah, Bhatia, Lyles, Ellinwood, \& Lee, 2009).

The present investigation demonstrates that the administration of bromocriptine (10 and $30 \mu \mathrm{g} / \mathrm{rat}$ ) following to hydro-alcoholic extract of ginseng $(100 \mathrm{mg} / \mathrm{kg} / \mathrm{d})$ for 7 days had an analgesic effect as great as ginseng alone in phases of formalin test. Therefore, ginseng extract may apply its analgesic effects through dopaminergic system receptors the same as dopamine. After the injection of a selective dopamine agonist in the nucleus accumbens core, it exerts analgesic effects of dopaminergic drugs in the formalin test (Faramarzi, Zendehdel, \& Haghparast, 2016). Haghparast, Ghalandari-Shamami, \& Hassanpour-Ezatti, (2012) suggested that dopamine D2 receptors in the nucleus accumbens had a critical role in the adjustment of acute and chronic inflammatory pain in the formalin test. Lintas et al. (2011) reported that bilateral microinjection of dopamine D2 receptor agonist in the nucleus accumbens inhibits the chronic phase caused by formalin test. 


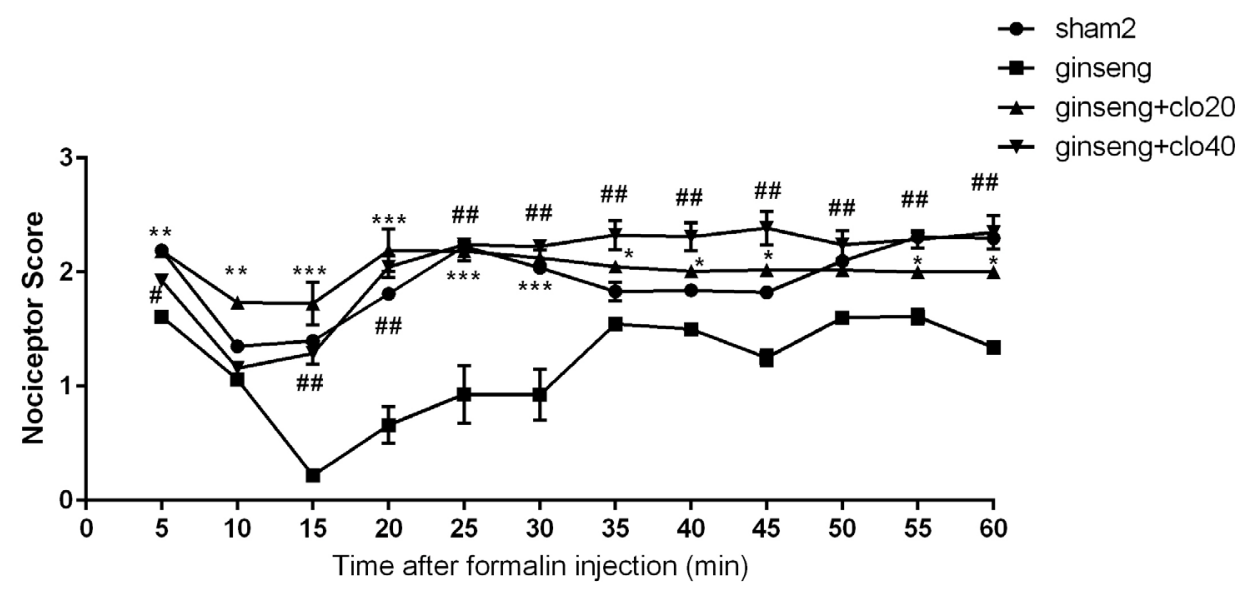

NEUR SCIENCE

Figure 3. Effect of clo (chlorpromazine) 20 and $40 \mu \mathrm{g} /$ rat on nociceptive scores (mean \pm SE) in rats pretreated by ginseng (100 $\mathrm{mg} / \mathrm{kg} / \mathrm{d}$ ) for 7 days

* Significant difference between clo 20 and $40 \mu \mathrm{g} /$ rat groups, in comparison to sham 2 group; \# Significant difference between clo 20 and $40 \mu \mathrm{g} / \mathrm{rat}$ groups, in comparison to ginseng $(100 \mathrm{mg} / \mathrm{kg} / \mathrm{d})$ group.

${ }^{*} \mathrm{P}<0.05,{ }^{* *} \mathrm{P}<0.01,{ }^{* *} \mathrm{P}<0.001 ; \# \mathrm{P}<0.05, \# \# \mathrm{P}<0.001$

There are several mechanisms for analgesic effects of bromocriptine; one of them pointed out that it prevents Nitric Oxide (NO) release by affecting $\alpha 2$-adrenergic receptors (Beck et al., 2004). Some investigations suggest that NO is a modulator of the nervous system in many activities. One of the NO roles is related to pain, because following nerve damages in the affected area, the level of NO increases there (Cury, Picolo, Gutierrez, \& Ferreira, 2011; Levy \& Zochodne, 2004). Moreover, the production of NO, which increases following the formalin test, is inhibited by flavonoids and phenolic compounds of ginseng, thereby decreasing NO that leads to analgesic activity (Jang et al., 2016; Kim et al., 2015). Therefore, ginseng extract and bromocriptine synergistically reduce NO production.

\section{Conclusion}

The current study shows that chlorpromazine in two doses following to IP injection of ginseng $(100 / \mathrm{mg} / \mathrm{kg} / \mathrm{d}) \mathrm{had}$ a hyperalgesic effect compared to the ginseng group. However, its hyperalgesic effect compared to the control and sham 2 groups is seen at $40 \mu \mathrm{g} /$ rat but not $20 \mu \mathrm{g} /$ rat dose.

Various studies show that the raclopride (dopamine D2 antagonist) causes hyperalgesia in rats and pet animals (Da Silva et al., 2017; Dias et al., 2015). Prescription of dopamine antagonists can inhibit the analgesic effect caused by the release of dopamine (Yazdi-Ravandi, Razavi, Haghparast, \& Goudarzvand, 2014). The selective prescription of dopamine D2 receptor antagonists systematically reduces the analgesic effect caused by amphetamines, morphine, and cocaine in the formalin test (Pelissier, Laurido, Hernandez, Constandil, \& Eschalier, 2006). Quinpirole as a dopamine D2-like receptor agonist reduces pain in both phases of the formalin test and sulpiride as an antagonist can potentially reverse analgesic effects observed by this agonist (Shamsizadeh, Pahlevani, Haghparast, Moslehi, \& Zarepour, 2013). These results supported our findings.

According to the present results, ginseng administration $(100 \mathrm{mg} / \mathrm{kg} / \mathrm{d})$ for 7 days had an analgesic effect, and bromocriptine administration in two different doses after pretreatment with ginseng $(100 \mathrm{mg} / \mathrm{kg} / \mathrm{d})$ had an analgesic effect the same as ginseng alone. Therefore, it seems that bromocriptine and ginseng had a synergistic but no additive effect. Finally, chlorpromazine administration in two different doses after pretreatment with ginseng $(100 \mathrm{mg} / \mathrm{kg} / \mathrm{d})$ had hyperalgesic effects compared to ginseng alone. Therefore, the hyperalgesic effect of chlorpromazine is more potent than the analgesic effect of ginseng.

\section{Ethical Considerations}

\section{Compliance with ethical guidelines}

All animal procedures were performed according to the Institutional Research Ethics Committee of the School of Veterinary Medicine of Shiraz University, Shiraz, Iran. 


\section{Funding}

This study was financially supported by Shiraz University (Grant: 94GCU4M1755).

\section{Authors' contributions}

Conceptualization, methodology, software, validation, formal analysis, investigation, resources, data curation, writing - original draft preparation, writing - review \& editing, visualization, supervision, and project administration, funding acquisition: Mahnaz Taherianfard; Methodology, investigation, resources, data curation: Somaye Aalam.

\section{Conflict of interest}

All authors declared no conflict of interest.

\section{References}

Beck, G., Brinkkoetter, P., Hanusch, C., Schulte, J., van Ackern, K., van der Woude, F. J., et al. (2004). Clinical review: Immunomodulatory effects of dopamine in general inflammation. Critical Care, 8(6), 485-91. [DOI:10.1186/cc2879] [PMID] [PMCID]

Becker, S., Ceko, M., Louis-Foster, M., Elfassy, N. M., Leyton, M., Shir, Y., et al. (2013). Dopamine and pain sensitivity: Neither sulpiride nor acute phenylalanine and tyrosine depletion have effects on thermal pain sensations in healthy volunteers. PLoS One, 8(11), e80766. [DOI:10.1371/journal.pone.0080766] [PMID] [PMCID]

Chang, Y. S., Seo, E. K., Gyllenhaal, C., \& Block, K. I. (2003). Panax ginseng: A role in cancer therapy? Integrative Cancer Therapies, 2(1), 13-33 [DOI:10.1177/1534735403251167] [PMID]

Cury, Y., Picolo, G., Gutierrez, V. P., \& Ferreira, S. H. (2011). Pain and analgesia: The dual effect of nitric oxide in the nociceptive system. Nitric Oxide, 25(3), 243-54 [DOI:10.1016/j.niox.2011.06.004] [PMID]

DaSilva, A. F., Nascimento, T. D., Jassar, H., Heffernan, J., Toback, R. L., Lucas, S., et al. (2017). Dopamine D2/D3 imbalance during migraine attack and allodynia in vivo. Neurology, 88(17), 1634-41 [DOI:10.1212/WNL.0000000000003861] [PMID] [PMCID]

Dauer, W., \& Przedborski, S. (2003). Parkinson's disease: Mechanisms and models. Neuron, 39(6), 889-909. [DOI:10.1016/S08966273(03)00568-3]

Dias, E. V., Sartori, C. R., Mariao, P. R., Vieira, A. S., Camargo, L. C., Athie, M. C., et al. (2015). Nucleus accumbens dopaminergic neurotransmission switches its modulatory action in chronification of inflammatory hyperalgesia. European Journal of Neuroscience, 42(7), 2380-9. [DOI:10.1111/ejn.13015] [PMID]

Faramarzi, G., Zendehdel, M., \& Haghparast, A. (2016). D1- and D2-like dopamine receptors within the nucleus accumbens contribute to stress-induced analgesia in formalin-related pain behaviours in rats. European Journal of Pain, 20(9), 1423-32. [DOI:10.1002/ejp.865] [PMID]

Garland, E. L. (2012). Pain processing in the human nervous system: A selective review of nociceptive and biobehavioral pathways. Primary Care, 39(3), 561-71. [DOI:10.1016/j.pop.2012.06.013] [PMID] [PMCID]

Haghparast, A., Ghalandari-Shamami, M., \& Hassanpour-Ezatti, M. (2012). Blockade of D1/D2 dopamine receptors within the nucleus accumbens attenuated the antinociceptive effect of cannabinoid receptor agonist in the basolateral amygdala. Brain Research, 1471, 23-32 [DOI:10.1016/j.brainres.2012.06.023] [PMID]

Jang, K. J., Choi, S. H., Yu, G. J., Hong, S. H., Chung, Y. H., Kim, C. H., et al. (2016). Anti-inflammatory potential of total saponins derived from the roots of Panax ginseng in lipopolysaccharideactivated RAW 264.7 macrophages. Experimental and Therapeutic Medicine, 11(3), 1109-15. [DOI:10.3892/etm.2015.2965] [PMID] [PMCID]

Jun, Y. L., Bae, C. H., Kim, D., Koo, S., \& Kim, S. (2015). Korean Red Ginseng protects dopaminergic neurons by suppressing the cleavage of p35 to p25 in a Parkinson's disease mouse model. Journal of Ginseng Research, 39(2), 148-54. [DOI:10.1016/j. jgr.2014.10.003] [PMID] [PMCID]

Kim, S. E., Shim, I., Chung, J. K., \& Lee, M. C. (2006). Effect of ginseng saponins on enhanced dopaminergic transmission and locomotor hyperactivity induced by nicotine. Neuropsychopharmacology, 31(8), 1714-21. [DOI:10.1038/sj.npp.1300945] [PMID]

Kim, Y. O., Kim, Y., Lee, K., Na, S. W., Hong, S. P., Valan Arasu, M., et al. (2015). Panax ginseng improves functional recovery after contusive spinal cord injury by regulating the inflammatory response in rats: An in vivo study. Evidence-Based Complementary and Alternative Medicine, 2015, 817096. [DOI:10.1155/2015/817096] [PMID] [PMCID]

Levy, D., \& Zochodne, D. W. (2004). No pain: Potential roles of nitric oxide in neuropathic pain. Pain Practice, 4(1), 11-18. [DOI:10.1111/j.1533-2500.2004.04002.x] [PMID]

Lintas, A., Chi, N., Lauzon, N. M., Bishop, S. F., Gholizadeh, S., Sun N., et al. (2011). Identification of a dopamine receptor-mediated opiate reward memory switch in the basolateral amygdala-nucleus accumbens circuit. The Journal of Neuroscience, 31(31), 11172 83. [DOI:10.1523/JNEUROSCI.1781-11.2011] [PMID] [PMCID]

Mancusoa, C., \& Santangelob, R. (2017). Panax ginseng and Panax quinquefolius: From pharmacology to toxicology. Food and Chemical Toxicology, 107(Part A), 362-72. [DOI:10.1016/j. fct.2017.07.019] [PMID] [PMCID]

Nabata, H., Saito, H., \& Takagi, K. (1973). Pharmacological studies of neutral saponins (GNS) of panax ginseng root. The Japanese Journal of Pharmacology, 23(1), 29-41 [DOI:10.1254/jjp.23.29] [PMID]

Nah, S. Y., Bhatia, K. S., Lyles, J., Ellinwood, E. H., \& Lee, T. H. (2009). Effects of ginseng saponin on acute cocaine-induced alterations in evoked dopamine release and uptake in rat brain nucleus accumbens. Brain Research, 1248, 184-90. [DOI:10.1016/j. brainres.2008.10.064] [PMID] [PMCID]

Nah, S. Y., \& McCleskey, E. W. (1994). Ginseng root extract inhibits calcium channels in rat sensory neurons through a similar path but different receptor, as mu-type opioids. Journal of Ethnopharmacology, 42(1), 45-51. [DOI:10.1016/0378-8741(94)90022-1] 
Palaniyandi, S. A., Suh, J. W., \& Yang, S. H. (2017). Preparation of Ginseng Extract with Enhanced Levels of Ginsenosides Rg1 and Rb1 Using High Hydrostatic Pressure and Polysaccharide Hydrolases. Pharmacognosy Magazine, 13(Suppl 1), S142-S147. [DOI:10.4103/0973-1296.203992] [PMID] [PMCID]

Pelissier, T., Laurido, C., Hernandez, A., Constandil, L., \& Eschalier, A. (2006). Biphasic effect of apomorphine on rat nociception and effect of dopamine D2 receptor antagonists. European Journal of Pharmacology, 546(1-3), 40-7. [DOI:10.1016/j.ejphar.2006.06.081] [PMID]

Rausch, W. D., Liu, S., Gille, G., \& Radad, K. (2006). Neuroprotective effects of ginsenosides. Acta Neurobiologiae Experimentalis Journal, 66(4), 369-75. [PMID]

Rhim, H., Kim, H., Lee, D. Y., Oh, T. H., \& Nah, S. Y. (2002). Ginseng and ginsenoside Rg3, a newly identified active ingredient of ginseng, modulate $\mathrm{Ca}_{2}{ }^{+}$channel currents in rat sensory neurons. European Journal of Pharmacology, 436(3), 151-158. [DOI:10.1016/ S0014-2999(01)01613-2]

Sandoval-Avila, S., Diaz, N. F., Gómez-Pinedo, U., CanalesAguirre, A. A., Gutiérrez-Mercado, Y. K., Padilla-Camberos, E., et al. (2018). Neuroprotective effects of phytochemicals ondopaminergic neuron cultures. Neurologia, 34(2), 114-24. [DOI:10.1016/j.nrleng.2016.04.014]

Shamsizadeh, A., Pahlevani, P., Haghparast, A., Moslehi, M., \& Zarepour, L. (2013). Involvement of dopamine receptors within the dorsal hippocampus in suppression of the formalin-induced orofacial pain. Pharmacology Biochemistry and Behavior, 114-115, 37-42. [DOI:10.1016/j.pbb.2013.10.029] [PMID]

Shin, Y. H., Kim, S. C., Han, J. W., Kim, D. H., Han, S. S., Shin, D. H., et al. (1997). Study on ginseng protopanaxadiol and protopanaxatriol saponins-induced antinociception. The Korean Journal of Physiology \& Pharmacology, 1(2), 143-9. https:/ / www. koreascience.or.kr/article/JAKO199703039540433.pdf

Sun, L. Q. (2004). Information on research and application of Ginseng; the king of traditional and herbal medicines. Asian Journal of Drug Metabolism and Pharmacokinetics, 4(4), 264-82. https://www.researchgate.net/publication/266372364

Volkow, N. D. (2010). Opioid-dopamine interactions: Implications for substance use disorders and their treatment. Biological Psychiatry, 68(8), 685-86. [DOI:10.1016/j.biopsych.2010.08.002] [PMID] [PMCID]

Yazdi-Ravandi, S., Razavi, Y., Haghparast, A., \& Goudarzvand, M. (2014). Orexin a induced antinociception in the ventral tegmental area involves D1 and D2 receptors in the nucleus accumbens. Pharmacology Biochemistry and Behavior, 126, 1-6 [DOI:10.1016/j.pbb.2014.08.009] [PMID] 
This Page Intentionally Left Blank 


\title{
Resaerch Paper: Association of CandidateSingle Nucleotide Polymorphisms Related to Candidate Genes in Patients With Schizophrenia
}

\author{
Seyedeh Sara Karimian ${ }^{*}$ (D, Mohammad Taghi Akbari ${ }^{2,3}$ (D), Seyed Saeed Sadr ${ }^{4}$, Gholamreza Javadi ${ }^{1}$ (D) \\ 1. Department of Biology, Science and Research Branch, Islamic Azad University, Tehran, Iran. \\ 2. Department of Medical Genetics, Faculty of Medical Sciences, Tarbiat Modarres University, Tehran, Iran \\ 3. Tehran Medical Genetics Laboratory, Tehran, Iran. \\ 4. Department of Psychiatry, Imam Hussein Hospital, Shahid Beheshti University, Tehran, Iran.
}

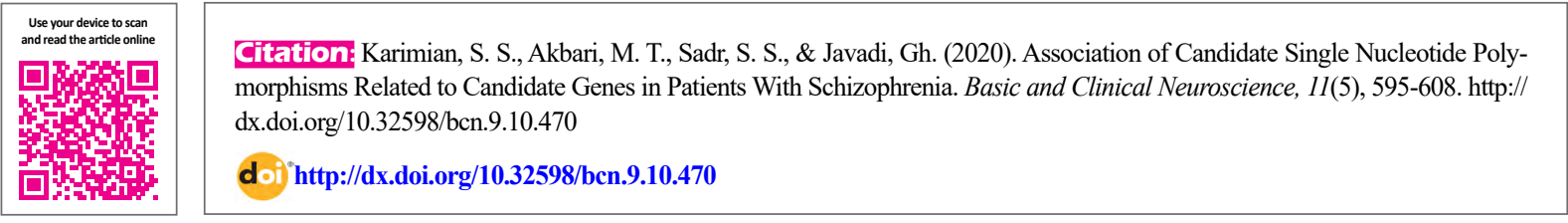

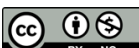

Article info:

Received: 28 May 2018

First Revision: 10 Jun 2018

Accepted: 06 Apr 2019

Available Online: 01 Sep 2020

Keywords:

Biomarkers, SNPs, NrCAM, PRODH, ANKK1, ANK3,

Schizophrenia

\section{A B S T RA C T}

Introduction: Schizophrenia is a chronic heterogenic neurodevelopment disorder. Many genes interfere in the development of SCZ. All four genes, NrCAM, PRODH, ANK3, and ANKK1, which were evaluated in this study, were previously reported to be associated with Schizophrenia. The NrCAM contributes to creating cognitive deficiencies through the CAM's signaling pathway. PRODH plays a vital role in creating SCZ negative symptoms through the signaling pathway of glutamatergic and NMDA receptors. ANK 3 affects ion channel and molecular adhesion in Ranvier and initial segments of axons, leading to mental retardation, sleep disorder, and SCZ. ANKK1 encodes a protein kinase and was reported to be associated with alcohol addiction, Attention Deficit Hyperactivity Disorder (ADHD), and SCZ.

Methods: The subjects were selected from Schizophrenic patients referring to the Psychiatric Ward of Imam-Hussein Hospital and Schizophrenic Patients Support Institution (AHEBBA). 95 (30 Schizoaffective patients, 57 Paranoid patients, and 8 disorganized) patients were recruited as the subjects in the present case-control association study. 120 healthy subjects were recruited from the Tehran Medical Genetics Laboratory staff and a group of students from the Islamic Azad University of Science and Research in Tehran. The genotypes were determined with molecular genotyping techniques of PCR-RFLP, ARMS-PCR, and Cycle sequencing. Results were analyzed by the Chi-Square test using SPSS V. 24 and R, SNP STATE Package to investigate significant differences between cases and controls.

Results: The incidence of schizophrenia was $68 \%$ and $32 \%$ among men and women, respectively. The evaluation of the allelic association between schizophrenia and all the candidate SNPs showed a significant association between NrCAM's SNP rs10235968 and SCZ ( $\mathrm{P}=0.001)$. Haplotype T, T, C in rs10235968, rs6967368, rs3763463, respectively, within the NrCAM gene, showed significant association with schizophrenia disorder $(\mathrm{P}=0.0001)$.

Conclusion: No association was found between other candidate SNPs and SCZ among the subjects.

* Corresponding Author:

Seyedeh Sara Karimian, PhD.

Address: Department of Biology, Science and Research Branch, Islamic Azad University, Tehran, Iran.

Tel: +98 (912) 3309710

E-mail:mtakbari@modares.ac.ir 


\section{Highlights}

- Schizophrenia (SCZ) is a chronic mental disorder.

- Many genes and environmental factors are involved in SCZ development.

- The CAM's signaling pathway is a candidate pathway involved in SCZ.

\section{Plain Language Summary}

Schizophrenia (SCZ) is a chronic mental disorder. Many genes and environmental factors are involved in SCZ development. The CAM's signaling pathway is a candidate pathway involved in SCZ to be linked to both psychosis and neurocognitive dysfunctions. Using GWAS and signaling pathways data, researchers reported several signaling pathways contributing to the development of SCZ. One of the most potent risk factors for SCZ is $22 \mathrm{q} 11$ deletion syndrome. The administration of NMDA antagonists results in cognitive deficiency and SCZ syndromes among healthy people, leading to dopaminergic neurons transmission. ANKK1 encodes a serine-threonine kinase protein and belongs to a receptor-interacting serine/threonine-protein kinase family, which contributes to cell division and cell differentiation. ANKK1 is located on the chromosome 11q32.2 and includes 10 exons. It was reported to be associated with alcohol addiction, eating disorder, SCZ, and Attention Deficit Hyperactivity Disorder (ADHD). This study aimed to investigate the association between the candidate SNPs within the NrCAM gene, and in the PRODH gene.

\section{Introduction}

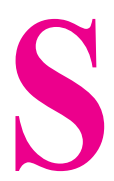

chizophrenia (SCZ) is a chronic mental disorder. Many genes and environmental factors are involved in SCZ development. NrCAM is a candidate gene for schizophrenia and has been reported in many studies with conflicting results (Ayalew, Le-Niculescu, et al. 2012). NrCAM encodes the neuron cells' adhesion protein molecule and is located on 7q31.1. The Northern Blotting technique showed a $2 \mathrm{~kb}$ transcript of $\mathrm{NrCAM}$ in all brain tissues. The $7.0 \mathrm{~kb}$ transcript of NrCAM is highly expressed in the brain medulla, adrenal, and adrenal cortex (Lane, et al., 1996, Wang, Williams, Du, Terrett, \& Kenwrick, 1998). NrCAM owns 36 exons and is involved in WNT and CAM's signaling pathways (Chen \& Zhou 2010, Zhang et al., 2015). NrCAM is one of the twelve genes active in CAM's pathway and has a significant relationship with schizophrenia. It acts as an interneurons connection and a signal transmission in CAM's pathway (Zhang et al., 2015).

The CAM's signaling pathway is a candidate pathway involved in SCZ, repeatedly reported to be linked with both psychosis and neurocognitive dysfunctions (Zhang et al., 2015). CAM's pathway plays a vital role in the brain's cognitive functions, including the formation of memory, attention, learning, reasoning, and thinking disrupted in SCZ (Hargreaves et al., 2014).
The gene NrCAM is active in interneurons, neuron-glial adhesion, and growth cone motility (Kamiguchi \& Lemmon, 1997). NrCAM has also been demonstrated to participate in many cellular processes of central and peripheral nervous systems, inclusive neurite growth, exon routing, myelination, and cellular migration (Kamiguchi \& Lemmon 1997).

Using GWAS and signaling pathways data, researchers reported several signaling pathways contributing to the development of SCZ, including ionic channel pathway (Askland, Read, O'Connell, \& Moore, 2012), myelination pathway (Yu et al., 2014), apoptosis factor pathway, adhesion molecule pathway, growth factor signaling pathway, and glutamate metabolism pathway (Jia, Wang, Meltzer, \& Zhao, 2010). Cell adhesion molecules are glycoproteins expressed on the cell surface and significantly contribute to biological processes, including immune responses, inflammation responses, embryonic development (Elangbam, Qualls Jr, \& Dahlgren, 1997). NrCAM is mostly reported about autism (Sakurai, et al., 2006).

NrCAM's SNPs rs3763463, rs10235968, rs6967368 are located in the promoter area, and rs1269634 is located in intron area. They were evaluated among an Iranian sample of patients with schizophrenia. An association study on NrCAM's SNPs and SCZ was conducted in the Korean population, and no association was found between NrCAM's SNPs and SCZ (Kim et al., 2009). 
In another study conducted at the University of California, NrCAM's SNP rs646558 showed association with SCZ (Atz, Rollins, \& Vawter, 2007). Yoo et al, evaluated the association between NrCAM's SNPs and personality disorder, addiction to amphetamines. Their results showed a significant association between NrCAM's SNP rs129634 and symptomatic amphetamine addiction (Yoo et al., 2012). One of the most potent risk factors for SCZ is 22q11 deletion syndrome (Levinson, et al., 2011). One-third of patients with $22 \mathrm{q} 11$ deletion syndrome (which is also called DiGeorge or Velocardiofacial syndrome) are suffering from Schizophrenia/Schizoaffective (Pulver et al., 1994). while the prevalence ratio of $22 \mathrm{q} 11$ deletion syndrome among the populations is $1 / 4000$, this rate is about $1 \%$ among schizophrenic cases (Christofolini et al., 2011). 22q11 deletion syndrome was proposed as a genetic kind of SCZ; therefore, all the sequences located in the 22q11 deletion area are addressed to be linked with schizophrenia. PRODH is one of the genes located on the chromosome 22q11.2 and includes 15 exons. PRODH encodes the Proline dehydrogenase enzyme and Catalyzing conversion of Proline into Glutamate. Hyperprolinemia is regarded as involved in the development of SCZ (Jacquet et al., 2002, Kempf et al., 2008). On the one hand, Proline is a modulator of glutamine neurotransmitters (Liu et al., 2002). In particular, the hypofunction of the NMDA receptor, a glutamatergic receptor, is also involved in the development of SCZ (Galderisi, Merlotti, \& Mucci, 2015).

The administration of NMDA antagonists results in cognitive deficiency and SCZ syndromes among healthy people, leading to dopaminergic neurons transmission (Galderisi, Merlotti, \& Mucci, 2015). The association of PRODH's SNP rs238731 located in the exon 12 and SCZ is supported in numerous studies (Shashi, Berry, \& Keshavan, 2009; Ota et al., 2014).

This study aimed to investigate the association between the candidate SNPs rs10235968, rs1269634, rs6967368, rs3763463 within the NrCAM gene, and rs2238731 in the PRODH gene in an Iranian sample of patients with schizophrenia.

ANKK1 encodes a serine-threonine kinase protein and belongs to a receptor-interacting serine/threonineprotein kinase family, which contributes to cell division and cell differentiation (Hamosh et al., 2002; Jasiewicz et al., 2014). ANKK1 is located on the chromosome $11 \mathrm{q} 32.2$ and includes 10 exons. ANKK1 was reported to be associated with alcohol addiction, eating disorder, SCZ, and Attention Deficit Hyperactivity Disorder (ADHD) (Jasiewicz et al., 2014; Arab \& Elhawary
2015). c.562C $>\mathrm{T}$ within the ANKK1 gene located in exon 3 argued to have a significant association with susceptibility to SCZ (Shirzad, Beyraghi, Ataei, \& Akbari, 2017). ANK 3 is located on chromosome 10q21.2, includes 52 exons, and was reported to be associated with mental retardation and SCZ (Iqbal et al., 2013). The protein encoded by ANK3 assumes to be connected to integral proteins and locates in the Ranvier node and axons (Iqbal et al., 2013). c.7649 G>T within ANK3 locates in the exon 13 was shown to influence SCZ susceptibility (Shirzad, Beiraghi, et al. 2017). Another goal of this study is to investigate the frequency of recurrence of two novel variants c.562 C > T and c.7649 G> T within genes ANKK1, ANK3 respectively, in the two groups Iranian case and control.

\section{Materials and Methods}

\subsection{Patients and controls}

This study was a case-control association study where none of the patients were relatives. A total of 95 patients (men=65, women=30) with average age $32 \pm 12.18$ were recruited from the Psychiatric ward of Imam Hussein Hospital and Schizophrenic Patient Support Institution (AHEBBA) in Tehran from August 2015 to May 2016. (30 Schizoaffective, 57 Paranoid, and 8 disorganized). The patients' demographic specifications, including gender, age, place of birth, and age at onset, were recorded. Patients were identified by drawing pedigree and interviews to recognize any physical illness or a family history of psychiatric disorders. Patients were clinically diagnosed with SCZ by an expert psychiatrist, according to the approach of Diagnostic and Statistical Manual of Mental Disorders (DSM-5) symbolism. Positive and Negative Syndrome Scale (PANSS) was used to recognize the severity of Positive and Negative Syndromes among schizophrenic cases.

A total of 120 healthy subjects $(\operatorname{men}=78$, women=42) with an average age of $35 \pm 14.14$ were recruited from Tehran Medical Genetics Laboratory staff and a group of students from Islamic Azad University of Science and Research in Tehran. These subjects were demographically matched by age and gender. They were identified by drawing pedigree and interviews to recognize any previous psychological or a family history of psychiatric disorders and physical diseases. Case and control groups were matched in terms of age and gender. None of the participants had a specific physical illness. The specifications of case and control subjects are introduced in Table 1. 
Table 1. Demographics and characteristics of cases and controls

\begin{tabular}{ccccc}
\hline \multirow{2}{*}{ Variable } & N & Frequency (\%) & N & Frequency (\%) \\
\cline { 2 - 5 } & \multicolumn{2}{c}{ SCZ Patients } & 120 & Controls \\
\hline Gender & 95 & $31.4 \%$ & 42 & $34.5 \%$ \\
\hline Female & 30 & $68.6 \%$ & 78 & $65.5 \%$ \\
\hline Male & 65 & $32 \pm 12.18$ & 120 & $35( \pm 14.14)$ \\
\hline Age & 95 & $21.43 \pm 7.32$ & & \\
\hline Age at onset & 80 & $31.1 \%$ & & \\
\hline Family History (\%) & 80 & $80 \pm 10.0039$ & & \\
\hline PANSS score & 80 & & & \\
\hline
\end{tabular}

NEUR SCIENCE

SCZ: schizophrenia, N: sample size, PANSS: positive and negative syndrome scale. As shown, 65 males and 30 females have participated in the case group with average age $32 \pm 12.18$, PANSS score with average score $80 \pm 10.0039$, and age at onset with average age $21.43 \pm 7.32$ And $31.1 \%$ of patients had a Family History of schizophrenia disorder. Also, among the controls group, 78 males and 42 females have participated with an average age $(35 \pm 14.14)$.

\subsection{Ethical considerations}

The consent Letter was taken from all patients and their caregivers. This research was conducted in agreement with the Declaration of Helsinki. This investigation was authorized by the medical ethics committee of the Science and Research Branch of Islamic Azad University of Tehran.

\subsection{DNA extraction}

The blood samples $(5 \mathrm{ml})$ were obtained from all cases and controls in EDTA tubes and were prepared for DNA extraction. In this study, MagCore HF16 Automatic Nucleic Acid Extractor system (RBC Bioscience Corp, Taiwan) with MagCore blood Genomic DNA Extraction Kit (RBC Bioscience Corp, Taiwan) was used to extracting of Genomic DNA samples. Then they were stocked at a temperature of $-20^{\circ} \mathrm{C}$. Nanodrop and $1 \%$ agarose gel electrophoresis were used to specify the quality and the quantity of the purified DNA.

\subsection{Primer design}

The software Gene Runner (www.SNPs.com) and primer-blast-NCBI-NIH databases were used to designing primers for PCR-RFLP, ARMS-PCR, and CycleSequencing methods.

SNPs rs3763463, rs2238731, and novel variant c.562 $\mathrm{C}>\mathrm{T}$ were genotyped through the PCR-RFLP method (Table 2). The PCR-RFLP primers were designed according to the recognition site and the fragments' length after enzymatic digestion. Remaining SNPs rs1269634, rs6967368, rs10235968, and novel variant c.7649 G>T were genotyped through the ARMS-PCR method (Table 3). Two forward primers and one reverse common primer were designed for any polymorphisms which were examined by ARMS-PCR (i.e. wild type [Wt] and mutant [mut]). A mismatch would appear in the $3^{\prime}$ terminal of mutant primer. However, more changes inside the last five nucleotides at the $3^{\prime}$ terminal of the mutant primer will lead to enhance the specificity (Little 1995; Table 3). A fragment of the $\beta$-globin gene was chosen as an internal control. Necessary primers for Cycle sequencing were designed for all the polymorphisms and were listed in Table 4.

\subsection{PCR-AFLP}

While Polymerase Chain Reaction-Restriction Fragment Length Polymorphism (PCR-RFLP) was used for genotyping target SNPs, it needs to be digested using certain restriction enzymes. Their recognition site and the length of fragments obtained from the enzymatic digestion are described in Table 5.

Samples were evaluated for wild and mutant alleles in each PCR run. Two tubes containing positive and negative control were replicated along with other tubes. The appropriate restriction enzymes in agreement with the manufacturer's instructions were used to digesting the PCR products. A $12 \%$ polyacrylamide gel electrophoresis and silver staining protocols were used to visualizing digested products. The PCR conditions for PCR-AFLP:

- Reaction volume: $\quad 20 \mu \mathrm{l}$

- PCR mix: $\quad 19.05 \mu \mathrm{l}$ 
Table 2. List of primers used for PCR-RFLP method.

\begin{tabular}{|c|c|c|c|c|}
\hline Gene & SNP & Primer sequences $\left(5^{\prime}-3^{\prime}\right)$ & $\begin{array}{l}\text { Product } \\
\text { length (bp) }\end{array}$ & $\begin{array}{l}\text { Restriction } \\
\text { Enzyme }\end{array}$ \\
\hline NRCAM & rs3763463 (C/G) & $\begin{array}{l}\text { Forward: 5'.. GCAGCAAGCAGTGTGTTTACTC.. 3' } \\
\text { Reverse: 5'. CTTCGAAATTCATCAGTTGGG...... 3' }\end{array}$ & $320 \mathrm{bp}$ & sstll \\
\hline PRODH & $\mathrm{rs} 2238731(\mathrm{G}>\mathrm{T})$ & $\begin{array}{l}\text { Forward: } 5^{\prime} \ldots . . \text { GGACAGAGGTTGGAGGCCC........ } 3^{\prime} \\
\text { Reverse: } 5^{\prime} \ldots \text { GTTGATGGGTCCTCATAGCC..... } 3^{\prime}\end{array}$ & 315 bp & Hin1II (NIallI) \\
\hline ANKK1 & c. $562 \mathrm{C}>\mathrm{T}$ & $\begin{array}{l}\text { Forward: 5'... ACCCTGGAACAGGCAGATAGC... 3' } \\
\text { Reverse: 5'.. GTTCACACAGTCCCAGGCAAG.... 3' }\end{array}$ & $444 \mathrm{bp}$ & Dde I \\
\hline
\end{tabular}

NEUR SCIENCE

Table 3. List of primers used for ARMS-PCR method

\begin{tabular}{|c|c|c|c|c|c|}
\hline Gene & SNP & & mer sequences $\left(5^{\prime}-3^{\prime}\right)$ & $\begin{array}{l}\text { Product } \\
\text { length (bp) }\end{array}$ & $\begin{array}{l}\mathrm{Tm} \\
{ }^{\circ} \mathrm{C}\end{array}$ \\
\hline NRCAM & $\begin{array}{l}\mathrm{rs} 1269634 \\
(\mathrm{~A} / \mathrm{G})\end{array}$ & $\begin{array}{l}\text { Forward (Wt): } \\
\text { Forward (Mut): } \\
\text { Reverse(C): }\end{array}$ & $\begin{array}{l}\text { 5'... GTTTAAGTAATTTTCATGCGCGA......... 3' } \\
\text { 5'... GTTTAAGTAATTTTCATGCGTGG..... 3' } \\
\text { 5'. TCATAAGGATGGTAGACAGATTATGC.. } \text { 3' }^{\prime}\end{array}$ & 260 bp & 58 \\
\hline NRCAM & rs6967368 (A/T) & $\begin{array}{l}\text { Forward(Wt): } \\
\text { Forward Mut: } \\
\text { Reverse(C): }\end{array}$ & $\begin{array}{l}5^{\prime} \ldots . . . \text { TCTTTTTTCATTTGGCAAACCCTT... 3' } \\
5^{\prime} \text {......TCTTTTTCATTTGGCAAACCCTA... 3' } \\
5^{\prime} \ldots . . . . \text { CATGGGAAGGACAGGCAAGAC.... } 3^{\prime}\end{array}$ & $331 \mathrm{bp}$ & 60 \\
\hline NRCAM & $\begin{array}{l}\text { rs10235968 } \\
\text { (C/T) }\end{array}$ & $\begin{array}{l}\text { Forward(Wt): } \\
\text { Forward(Mut): } \\
\text { Reverse(C): }\end{array}$ & 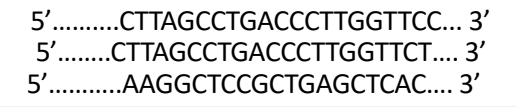 & $126 \mathrm{bp}$ & 62 \\
\hline ANK3 & c.7649 G>T & $\begin{array}{l}\text { Forward(Wt): } \\
\text { Forward (Mut): } \\
\text { Reverse (C): }\end{array}$ & $\begin{array}{l}5^{\prime} \ldots . . . \text {.CATCCACATGGCATGTTTTAGAC.... 3' } \\
5^{\prime}, \ldots . . . \text { CATCCACATGGCATGTTTTAGAA... 3' } \\
5^{\prime} \ldots . . . \text { GAGTCATTGCCTTCTTATCTGGAG... 3' }\end{array}$ & $471 \mathrm{bp}$ & 58 \\
\hline $\begin{array}{l}\beta \text { - globin gene } \\
\text { (Internal } \\
\text { control) }\end{array}$ & & \multicolumn{2}{|c|}{$\begin{array}{l}\text { Forward Control primer: } 5^{\prime} \text { '.....CAATGTATCATGCCTCTTTGCACC... 3' } \\
\text { Reverse Control primer: } 5^{\prime} \text { '...GAGTCAAGGCTGAGAGATGCAGGA... } 3^{\prime}\end{array}$} & $800 \mathrm{bp}$ & \\
\hline
\end{tabular}

NEUR SCIENCE

Forward (Wt): Forward wild type primer, Forward Mut: Forward mutant primer, Reverse (C): Reverse common primer

- Primer concentration: $0.15 \mu \mathrm{l}$ (each forward and reverse)

- Taq polymerase:

$0.15 \mu \mathrm{l}$

- Template DNA:

$0.5 \mu \mathrm{l}(\sim 16.5 \mathrm{ng})$

The thermal cycling:

- Primary denaturation: $\quad 5$ minute at $95^{\circ} \mathrm{C}$

- No. of cycles:

30

- Denaturation:

30 seconds at $94^{\circ} \mathrm{C}$

- Annealing:

30 seconds at $61^{\circ} \mathrm{C}$

- Extension:

30 seconds at $72^{\circ} \mathrm{C}$

- Terminal extension: $\quad 10$ minute at $72^{\circ} \mathrm{C}$
The enzymatic digestion conditions for digestion of PCR products directly after amplification:

- Add:

PCR reaction mix: $\quad 5 \mu \mathrm{l}$

Nuclease-free water: $\quad 4.7 \mu \mathrm{l}$

10xBuffer G: $\quad 1 \mu \mathrm{l}$

Restriction enzyme: $\quad 0.3 \mu \mathrm{l}$

- Mix softly and spin down for a few second

- Incubate at $37^{\circ} \mathrm{C}$ for over night

Thermal inactivation: Restriction enzyme is inactivated by incubation at $65^{\circ} \mathrm{C}$ for $20 \mathrm{~min}$. 
Table 4. List of primers used for cycle sequencing method.

\begin{tabular}{|c|c|c|c|c|}
\hline Gene & SNPs & Primer sequences (5'-3') & $\begin{array}{l}\text { Product } \\
\text { length (bp) }\end{array}$ & Tm \\
\hline NrCAM & rs1269634 (A/G) & $\begin{array}{l}\text { Forward: } 5^{\prime} \ldots . . \text { GGATGGTAGACAGATTATGCTTCA ... 3' } \\
\text { Reverse: } 5^{\prime} . . . . \text { GCAGTTCAGAGTGATGATAAATGC.... 3' }\end{array}$ & 515 bp & 58 \\
\hline NrCAM & rs6967368 (A/T) & $\begin{array}{l}\text { Forward: 5'.. AATCTGCTCCTAACTTATCTCTCCATT... 3' } \\
\text { Reverse: 5'. AAATTGTCCTCAAAGAAGTGAAATTTT.. 3' }\end{array}$ & $236 \mathrm{bp}$ & 59 \\
\hline \multirow[b]{2}{*}{ NrCAM } & rs3763463 (C/G) & $\begin{array}{c}\text { Forward: } 5 ' \ldots . . . . . \text { GCAGCAAGCAGTGTGTTTACTC....... 3' } \\
\text { Reverse: } 5^{\prime} \ldots . . . . . \text { CTTCGAAATTCATCAGTTGGG....... 3' }\end{array}$ & $320 \mathrm{bp}$ & 60 \\
\hline & rs10235968 (C/T) & $\begin{array}{l}\text { Forward:5'..... TGGTGAGGAGCTCAGAAAATGTT...... 3' } \\
\text { Reverse:5'.. ATTTGCTTATTTACAAATGGGGGAGTA.. 3' }\end{array}$ & $353 \mathrm{bp}$ & 60 \\
\hline PRODH & rs2238731(G/T) & $\begin{array}{l}\text { Forward: 5'.......... GGACAGAGGTTGGAGGCCC........ 3' } \\
\text { Reverse: } 5^{\prime} \ldots \ldots \ldots \text { GTTGATGGGGTCCTCATAGCC......... 3' }\end{array}$ & $315 \mathrm{bp}$ & 61 \\
\hline ANKK1 & c.562 C>T & $\begin{array}{l}\text { Forward: 5'.......ACCCTGGAACAGGCAGATAGC........ 3' } \\
\text { Reverse: } 5^{\prime} \ldots \ldots . . . \text { GTTCACACAGTCCCAGGCAAG........ 3' }\end{array}$ & $444 \mathrm{bp}$ & 61 \\
\hline ANK3 & c. $7649 \mathrm{G}>\mathrm{T}$ & $\begin{array}{l}\text { Forward: 5'......... GGGTCTGATAAGCGGTCCAG........ 3' } \\
\text { Reverse: 5'..........ACCATTTTTAGGGCGTGCC.......... 3' }\end{array}$ & $366 \mathrm{bp}$ & 59 \\
\hline
\end{tabular}

NEUR SCIENCE

- Electrophoresis: $10 \times 10 \mathrm{~cm} \mathrm{12 \%} \mathrm{polyacrylamide,} 1$ hour at 150 volts

- Staining: $\quad 0.1 \%$ silver nitrate.

\subsection{ARMS-PCR}

ARMS-PCR was performed in triplicates for all specimens. The results showed Wt [1] allele or Mut [2] allele, depending on the amplicon bands detected on a polyacrylamide gel. Samples carrying the $\mathrm{Wt} / \mathrm{Wt}$ alleles or Mut/ Mut alleles showed a single band on a polyacrylamide gel, while two bands with the same molecular size were expected for samples carrying both Mut and Wt alleles. A real negative is suggested by applying to replicate the internal control region and lack of amplification through the ARMS primers. The $12 \%$ polyacrylamide gel electrophoresis and silver staining protocols were used to visualizing amplified products [1], Wild type allele, and [2] Mutant type allele.

The PCR conditions for ARMS-PCR are:

- Reaction volume: $\quad 20 \mu \mathrm{l}$

- PCR mix: $\quad 18.21 \mu$

- Primer concentration: $0.25 \mu \mathrm{l}$ (each forward (Mut/ $\mathrm{Wt}$ ) and reverse); $0.07 \mu \mathrm{l}$ (each forward and reverse for $\beta$-globin)

- Taq polymerase: $\quad 0.15 \mu \mathrm{l}$

- Template DNA: $\quad 1 \mu \mathrm{l}(\sim 13 \mathrm{ng})$

Thermal cycling:

Table 5. Restriction enzymes and their recognition site

\begin{tabular}{|c|c|c|c|c|c|}
\hline \multirow[b]{2}{*}{ SNP } & \multirow[b]{2}{*}{ Restriction Enzyme } & \multirow[b]{2}{*}{ Recognition Site } & \multicolumn{3}{|c|}{ The length of the fragments } \\
\hline & & & Wild Type & Heterozygote & $\begin{array}{c}\text { Polymorphic } \\
\text { Type }\end{array}$ \\
\hline rs2238731 & Hin1 II (Nia III) & $\begin{array}{l}5^{\prime} \ldots \uparrow C A T G \downarrow \ldots 3^{\prime} \\
3^{\prime} \ldots \uparrow \text { T T A } \downarrow \\
\ldots\end{array}$ & $317 n t$ & $112 \mathrm{nt}, 205 \mathrm{nt}, 317 \mathrm{nt}$ & $205 n t, 112 n t$ \\
\hline rs3763463 & Sacll & $\begin{array}{l}5^{\prime} \ldots . \mathrm{CC} G C \downarrow \text { G } \ldots \ldots . .3^{\prime} \\
3^{\prime} \ldots \mathrm{GG} \uparrow C \mathrm{CC} \ldots \ldots .5^{\prime}\end{array}$ & $168 \mathrm{nt}, 152 \mathrm{nt}$ & $320 n t, 168 n t, 152 n t$ & $320 n$ \\
\hline rs897218854 & Ddel & $\begin{array}{l}5^{\prime} \ldots . . . C \downarrow T N A G \ldots \ldots .3^{\prime} \\
3^{\prime} \ldots . . . G A N T \uparrow C \ldots \ldots .5^{\prime}\end{array}$ & $\begin{array}{l}\text { 182nt, } 16 \mathrm{nt} \\
246 \mathrm{nt}\end{array}$ & $\begin{array}{c}\text { 198nt, } 246 \mathrm{nt}, 16 \mathrm{nt}, \\
182 \mathrm{nt}\end{array}$ & $198 n t, 246 n t$ \\
\hline
\end{tabular}

SNP: Single nucleotide polymorphism

NEUR SCIENCE 
Table 6. Genotype distribution for the candidate SNPs in the study

\begin{tabular}{|c|c|c|c|}
\hline \multicolumn{2}{|c|}{ Gene } & \multirow{2}{*}{$\begin{array}{c}\text { SNPs } \\
\text { SCZ }\end{array}$} & \multirow{2}{*}{$\begin{array}{c}\mathbf{P} \\
0.82\end{array}$} \\
\hline & & & \\
\hline NrCAM & rs 10235968 & Controls & 0.016 \\
\hline & & All subject & 0.093 \\
\hline \multirow{3}{*}{ NrCAM } & & SCZ & 0.83 \\
\hline & rs1269634 & Controls & 1 \\
\hline & & All subject & 0.78 \\
\hline \multirow{3}{*}{ NrCAM } & & SCZ & 0.22 \\
\hline & rs6967368 & Controls & 0.073 \\
\hline & & All subject & 0.034 \\
\hline \multirow{3}{*}{ NrCAM } & & SCZ & 1 \\
\hline & rs3763463 & Controls & 0.6 \\
\hline & & All subject & 0.71 \\
\hline \multirow{3}{*}{ PRODH } & & SCZ & 1 \\
\hline & rs2238731 & Controls & 1 \\
\hline & & All subject & 1 \\
\hline
\end{tabular}

NEUR SCIENCE

- Primary denaturation $\quad 5$ minute at $95^{\circ} \mathrm{C}$

- No. of cycles: 30

- Denaturation: 30 seconds at $94^{\circ} \mathrm{C}$

- Annealing: 30 seconds at $65^{\circ} \mathrm{C}$

- Extension: 30 seconds at $72^{\circ} \mathrm{C}$

- Terminal extension: $\quad 10$ minute at $72^{\circ} \mathrm{C}$

- Electrophoresis: 10x10 $\mathrm{cm} \mathrm{12 \%} \mathrm{polyacrylamide,} 1$ hour at 150 volts

- Staining: $0.1 \%$ silver nitrate

\subsection{Sequencing}

To confirm the results obtained by ARMS-PCR and PCR-AFLP, Applied Biosystems incorporation (ABI) and 3130 Genetic Analyzer were used to sequencing one-third of samples.

The PCR conditions for Sequencing:

- Reaction volume: $\quad 20 \mu \mathrm{l}$
- PCR mix:
$19.05 \mu 1$

- Primer concentration: $0.15 \mu \mathrm{l}$ (each forward and reverse),

- Taq polymerase: $\quad 0.15 \mu \mathrm{l}$

- Template DNA: $\quad 0.5 \mu \mathrm{l}(\sim 20 \mathrm{ng})$

The thermal cycling:

- Primary denaturation $\quad 5$ minute at $95^{\circ} \mathrm{C}$

- No. of cycles: $\quad 30$

- Denaturation: $\quad 30$ seconds at $94^{\circ} \mathrm{C}$

- Annealing: $\quad 30$ seconds at $65^{\circ} \mathrm{C}$

- Extension: $\quad 30$ seconds at $72^{\circ} \mathrm{C}$

- Terminal extension: $\quad 10$ minute at $72^{\circ} \mathrm{C}$

The PCR products were transferred to the advanced genomic department of Tehran Medical Genetic Laboratory for cycle sequencing. The cycle sequencing was implemented based on Applied Biosystem. 
TheApplied Biosystem Protocol:

- $96^{\circ} \mathrm{C}$ for $1 \mathrm{~min}$

- 25 cycles of:

- $96^{\circ} \mathrm{C}$ for 10 seconds.

- $50^{\circ} \mathrm{C}$ for 5 seconds.

- $60^{\circ} \mathrm{C}$ for 4 minute

- $4^{\circ} \mathrm{C}$ - hold

EDTA-Ethanol protocol was used for purifying products. After adding $12 \mu \mathrm{l}$ Formamide, purified products were denatured at $95^{\circ} \mathrm{C}$ for 5 minutes and then cooled off to $4^{\circ} \mathrm{C}$. Finally, products were run using the $3130 \mathrm{Ge}$ netic Analyzer.

\subsection{Statistical analysis}

The Chi-square test was used to determine departure from Hardy-Weinberg Equilibrium (HWE) for all SNPs (Wittke-Thompson, Pluzhnikov, \& Cox, 2005; Rodriguez, Gaunt, \& Day, 2009). To evaluate normality distributions, the Kolmogorov-Smirnov test using SPSS v. 24 was conducted. For analyzing the results, the Chi-square test using the R, SNP STATE Package to investigate the association of the candidate SNPs with SCZ. The Allele homozygosity and heterozygosity were specified. The OR (Odds Ratio) and 95\% CI (Confidence Interval) were determined for all genotypes. Calculated Probability $\mathrm{P} \leq 0.05$ was considered significant.

\section{Results}

\subsection{Hardy-Weinberg disequilibrium}

The genotype distribution for all candidate SNPs, among case and control, did not deviate from those predicted by Hardy-Weinberg ( $\mathrm{P}>0.05)$, except for SNP rs 10235968 in $\mathrm{NrCAM}$, in control group $(\mathrm{P}=0.016)$ and, SNP rs6967368 in $\mathrm{NrCAM}$ in all subject (case+control) $(\mathrm{P}=0.034)$. The $\mathrm{P}$ value for genotype distribution was calculated for the candidate SNPs and listed in Table 6. The rest of the candidate SNPs did not pervert from Hardy-Weinberg equilibrium in both case and control groups.

\subsection{Allele and genotype frequency of the candidate SNPs}

Incidence of Allele $\mathrm{C}$ and Allele $\mathrm{T}$ for NrCAM's SNP rs10235968 among patients was 0.66 and 0.34 , respec- tively, while the incidence of allele $\mathrm{C}$ and $\mathrm{T}$ was equal among the healthy group $(0.5 / 0.5)$. Genotyping frequency of two genotypes CC and TT for SNP rs10235968 among patients was 0.43 and 0.11 , respectively, while the frequency for the two genotypes among the healthy group was both 0.19 . The genotype and allele frequencies of all candidate SNPs were presented in Table 7.

Table 9 shows genotype association of SNP rs 10235967 in NrCAM with schizophrenia. In codominant inheritance: the genotype CT, 3.01 times, will increase the risk of schizophrenia, compared to the genotype CC. The genotype TT 4.1 times will increase the risk of schizophrenia compared to the genotype $\mathrm{CC}(\mathrm{P}=0.0006)$. In dominant inheritance: the genotypes $\mathrm{CT}+\mathrm{TT}, 3.21$ times will increase the risk of schizophrenia, compared to the genotype $\mathrm{CC}(\mathrm{P}=0.0001)$. In overdominant inheritance: the genotype CT, 1.87 times, will increase the risk of schizophrenia, compared to the genotypes $\mathrm{CC}+\mathrm{TT}$ $(\mathrm{P}=0.025)$.

\subsection{Haplotype analysis}

According to the haplotype analysis, shown in Table 10 , three alleles, $\mathrm{T}, \mathrm{T}$, and $\mathrm{C}$, in the variants rs 10235968 , rs6967368, rs3763463, respectively, within the $\mathrm{NrCAM}$ gene showed a strong association with schizophrenia disorder $(\mathrm{P}=0.0001)$.

\subsection{Linkage disequilibrium (LD)}

The SNPs rs10235968, rs6967368, rs3763463 located in the $\mathrm{NrCAM}$ gene promoter (Upstream variant $2 \mathrm{~KB}$ ) were analyzed for LD. NrCAM displayed LD in two blocks rs69677368 and rs3763463 ( $\mathrm{P}=0.0371)$.

\subsection{Frequency of recurrence of two novel variants c.562 $\mathrm{C}>\mathrm{T}$ and c.7649 $\mathrm{G}>\mathrm{T}$}

After genotyping two novel variants, c.562 $\mathrm{C}>\mathrm{T}$ and c.7649 G> T within genes ANKK1, ANK3, among patients and healthy people. We have found no recurrence frequency of two novel variants, c. $562 \mathrm{C}>\mathrm{T}$ and c. $7649 \mathrm{G}>\mathrm{T}$.

\section{Discussion}

According to the results of the present study and other studies investigating NrCAM's SNPs and schizophrenia association, all SNPs' frequency in this study has shown no significant association between the candidate SNPs and SCZ except for SNP rs10235968 in NrCAM, which was significantly associated with SCZ. It seems that the allele T in rs10235968 associated with schizophrenia, suggested be- 
Table 7. Genotype and allele frequencies of the all candidate SNPs

\begin{tabular}{|c|c|c|c|c|c|c|c|c|}
\hline \multirow{2}{*}{\multicolumn{2}{|c|}{ Gene }} & \multirow{4}{*}{$\begin{array}{c}\text { Polymorphisms } \\
\text { rs10235968 }\end{array}$} & \multirow{3}{*}{$\begin{array}{c}\mathbf{N} \\
95\end{array}$} & \multicolumn{3}{|c|}{ Genotype frequency (\%) } & \multicolumn{2}{|c|}{ Allele frequency } \\
\hline & & & & CC & CT & TT & C & $\mathbf{T}$ \\
\hline \multirow{2}{*}{ NrCAM } & SCZ & & & $41(43)$ & $44(46)$ & $10(11)$ & 0.66 & 0.34 \\
\hline & Controls & & 119 & $22(19)$ & $71(62)$ & $22(19)$ & 0.5 & 0.5 \\
\hline \multirow{2}{*}{\multicolumn{2}{|c|}{ Gene }} & \multirow{2}{*}{ Polymorphisms } & \multirow{2}{*}{$\mathbf{N}$} & \multicolumn{3}{|c|}{ Genotype frequency (\%) } & \multicolumn{2}{|c|}{ Allele frequency } \\
\hline & & & & AA & AG & GG & A & G \\
\hline \multirow{2}{*}{ NrCAM } & SCZ & \multirow{2}{*}{ rs1269634 } & 95 & $17(18)$ & $49(52)$ & $29(13)$ & 0.44 & 0.56 \\
\hline & Controls & & 119 & $16(13)$ & $56(47)$ & $47(39)$ & 0.37 & 0.63 \\
\hline \multirow{2}{*}{\multicolumn{2}{|c|}{ Gene }} & \multirow{2}{*}{ Polymorphisms } & \multirow{2}{*}{$\mathbf{N}$} & \multicolumn{3}{|c|}{ Genotype frequency (\%) } & \multicolumn{2}{|c|}{ Allele frequency } \\
\hline & & & & TT & TA & AA & A & $\mathbf{T}$ \\
\hline \multirow{2}{*}{ NrCAM } & SCZ & \multirow{2}{*}{ rs6967368 } & 95 & $70(74)$ & $21(22)$ & $4(3)$ & 0.15 & 0.85 \\
\hline & Controls & & 117 & $92(79)$ & $21(18)$ & $4(3.4)$ & 0.12 & 0.88 \\
\hline \multirow{2}{*}{\multicolumn{2}{|c|}{ Gene }} & \multirow{2}{*}{ Polymorphisms } & \multirow{2}{*}{$\mathbf{N}$} & \multicolumn{3}{|c|}{ Genotype frequency (\%) } & \multicolumn{2}{|c|}{ Allele frequency } \\
\hline & & & & CC & GC & GG & C & G \\
\hline \multirow{2}{*}{ NrCAM } & SCZ & \multirow{2}{*}{ rs3763463 } & 95 & $76(80)$ & 18(19) & $1(1)$ & 0.89 & 0.11 \\
\hline & Controls & & 118 & $94(80)$ & $24(20)$ & $0(0)$ & 0.9 & 0.1 \\
\hline \multirow{2}{*}{\multicolumn{2}{|c|}{ Gene }} & \multirow{2}{*}{ Polymorphisms } & \multirow{2}{*}{$\mathbf{N}$} & \multicolumn{3}{|c|}{ Genotype frequency (\%) } & Alle & ency \\
\hline & & & & CC & CT & TT & C & $\mathbf{T}$ \\
\hline 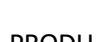 & SCZ & רברו & 95 & $93(98)$ & $2(2)$ & $0(0)$ & 0.99 & 0.01 \\
\hline PNUD & Controls & | & 119 & $117(98)$ & $2(1.7)$ & $0(0)$ & 0.99 & 0.01 \\
\hline & & Dolumomhirms & $M$ & Ger & ve freque & & Alle & ency \\
\hline & & roiymítiomis & No & CC & CT & TT & C & $\mathbf{T}$ \\
\hline$\triangle N K K 1$ & SCZ & $r c 897218854$ & 95 & $94(98.9)$ & $1(1.1)$ & $0(0)$ & 0.99 & 0.005 \\
\hline AIVNA & Controls & $13007<10034$ & 114 & $114(96.6)$ & $0(0)$ & $0(0)$ & 1 & 0 \\
\hline & & Polymornhisms & $N$ & Ger & ve freque & & Alle & ency \\
\hline & & 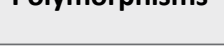 & 10 & GG & GT & TT & G & $\mathbf{T}$ \\
\hline 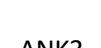 & SCZ & 61, & 95 & 94(98.9) & $1(1.1)$ & $0(0)$ & 0.99 & 0.005 \\
\hline Mivis & Controls & 0.104501 & 114 & $114(96.6)$ & $0(0)$ & $0(0)$ & 1 & 0 \\
\hline
\end{tabular}

N: Sample size, SCZ: Schizophrenia

NEUR SCIENCE

Table 8. Association between the candidate SNPs and schizophrenia

\begin{tabular}{cccccccc}
\hline Gene & Chr & SNP/Mutation & A1/A2 & Common allele & P Value & OR (95\%Cl) & $\mathbf{x}^{\mathbf{2}}$ \\
\hline NrCAM & 7 & rs10235968 & C/T & C & 0.001 & $1.969(1.323-2.929)$ & 11.284 \\
NrCAM & 7 & rs126934 & A/G & A & 0.144 & $1.407(0.88-2.22)$ & 2.134 \\
NrCAM & 7 & rs6967368 & T/A & T & 0.593 & $0.831(0.42-1.63)$ & 0.285 \\
NrCAM & 7 & rs3763463 & G/C & C & 0.781 & $0.817(0.05-13.28)$ & 0.078 \\
PRODH & 22 & rs2238731 & T/C & C & 0.881 & $0.81(0.05-13.02)$ & 0.022 \\
\hline
\end{tabular}


Table 9. Genotypic association of SNP rs10235967 in NrCAM with schizophrenia

\begin{tabular}{|c|c|c|c|c|c|}
\hline \multicolumn{6}{|c|}{ rs10235968 genotype association with SCZ } \\
\hline Model & Genotype & Case & Control & OR (95\% Cl) & P-value \\
\hline \multirow{3}{*}{ Codominant } & $\mathrm{C} / \mathrm{C}$ & $41(43.2 \%)$ & $22(19.1 \%)$ & 1.00 & \multirow{3}{*}{$6 e-04$} \\
\hline & $\mathrm{C} / \mathrm{T}$ & $44(46.3 \%)$ & $71(61.7 \%)$ & $3.01(1.59-5.70)$ & \\
\hline & $T / T$ & $10(10.5 \%)$ & $22(19.1 \%)$ & $4.10(1.65-10.18)$ & \\
\hline \multirow{2}{*}{ Dominant } & $\mathrm{C} / \mathrm{C}$ & $41(43.2 \%)$ & $22(19.1 \%)$ & 1.00 & \multirow{2}{*}{$1 e-04$} \\
\hline & $\mathrm{C} / \mathrm{T}-\mathrm{T} / \mathrm{T}$ & $54(56.8 \%)$ & $93(80.9 \%)$ & $3.21(1.73-5.95)$ & \\
\hline \multirow{2}{*}{ Recessive } & $\mathrm{C} / \mathrm{C}-\mathrm{C} / \mathrm{T}$ & 85 (89.5\%) & 93 (80.9\%) & 1.00 & \multirow{2}{*}{0.08} \\
\hline & $T / T$ & $10(10.5 \%)$ & $22(19.1 \%)$ & $2.01(0.90-4.49)$ & \\
\hline \multirow{2}{*}{ Over dominant } & $\mathrm{C} / \mathrm{C}-\mathrm{T} / \mathrm{T}$ & $51(53.7 \%)$ & $44(38.3 \%)$ & 1.00 & \multirow{2}{*}{0.025} \\
\hline & $\mathrm{C} / \mathrm{T}$ & 44 (46.3\%) & 71 (61.7\%) & $1.87(1.08-3.25)$ & \\
\hline
\end{tabular}

NEUR SCIENCE

SNP: Single Nucleotide Polymorphism, OR: Odds Ratio, CI: Confidence Interval, P-value: A small p-value (typically $\leq 0.05$ ) shows strong evidence against the null hypothesis, so the null hypothesis is rejected.

Table 10. Haplotype Analysis of the candidate SNPs

\begin{tabular}{ccccccc}
\hline \multicolumn{7}{c}{ Haplotype Association With SCZ } \\
\hline & rs10235968 & rs6967368 & rs3763463 & Frequency & OR (95\% Cl) & P-value \\
\hline 1 & C & T & C & 0.4544 & 1.00 & -- \\
\hline 2 & T & T & C & 0.3104 & $3.70(2.08-6.57)$ & $<0.0001$ \\
\hline 3 & T & A & C & 0.1169 & $1.31(0.67-2.54)$ & 0.43 \\
4 & C & T & G & 0.0984 & $1.68(0.79-3.58)$ & 0.18 \\
\hline 5 & C & A & C & 0.0151 & $4.66(0.46-47.20)$ & 0.19 \\
\hline
\end{tabular}

Global haplotype association p-value: 0.00015

NEUR:SCIENCE

ing risk in the Iranian population $(\mathrm{P}=0.001)$. The decreased frequency of genotype TT for SNP rs10235968 in NrCAM was seen among the patient's group (Table 7). Reduced homozygous CC and TT and increased heterozygote CT were observed among the healthy group in NrCAM's SNP rs10235968 (Table 7).

The Haplotype T, T, C in rs10235968, rs6967368, rs3763463, respectively, within the NrCAM gene, showed significant association with schizophrenia disor$\operatorname{der}(\mathrm{P}=0.0001)$. rs10235968 deviated from those predicted by Hardy-Weinberg equilibrium in the control group $(\mathrm{P}=0.016)$. Probably, it is because of the small sample size. Many studies have been conducted aim to genotype rs10235968. However, the results are a little different in different populations. According to the NCBI dataset, the common allele for SNP rs10235968 is C with allelic frequency 0.52 . in 1000 genome projects, the allele frequency of $\mathrm{C}$ and $\mathrm{T}$ was 0.521 and 0.479 , respectively. Nevertheless, in Africa, the frequency of allele $\mathrm{C}$ of rs10235968 is 0.446 , and allele $\mathrm{T}$ is 0.554 . The allele $\mathrm{C}$ may probably not be regarded as an ancestral allele, and more probably, rs10235968 is a balancing polymorphism.
The prominent role of SNP rs10235968 in the development of schizophrenia is still unknown. Although the candidate SNPs have been previously studied about SCZ, no study has been conducted on the association of these SNPs with SCZ among Iranian patients.

Two types of research evaluated the association of NrCAM's SNPs with SCZ: Atz, Rollins, \& Vawter (2007) in California University reported the association of SNP rs646558 in NrCAM with the susceptibility to SCZ, and Kim et al. (2009) evaluated the association of 13 SNPs in NrCAM and SCZ among Korean population and no association was found between the examined polymorphisms and SCZ. The latter study's Korean population results are not in line with those drawn from this study among Iranian patients. SNP rs10235968 is located in the promoter of NrCAM. The role of nucleotide changes in the upstream region of $\mathrm{NrCAM}$ is not entirely known. This region is not translated; however, since it is located in the gene's promoter region, the epigenetic changes may affect gene translation (Barbeau, Liang, Robitalille, Quirion, \& Srivastava, 1995). 
The NrCAM gene expression strongly decreases in the the brain of patients with SCZ, and the abnormal ratio of the synaptic NrCAM proteins found in the hippocampus of schizophrenic patients (Honer et al., 1997; Vawter, Howard, Hyde, Kleinman, \& Freed, 1999; Vawter, 2000). The association of CAM's signaling pathway and SCZ among Chinese and European populations were previously reported (O'Dushlaine et al., 2011, Zhang, et al., 2015). NrCAM is involved in CAM's signaling pathway, which has an essential role in the brain's cognitive function, an attribute that is disrupted in SCZ. It is hypothesized that the SNPs of NrCAM can affect CAM's pathways; by how they can affect the brain's cognitive function. NrCAM SNPs are likely to affect the function of the protein or gene expression, through which they can affect the process of transmitting signals between neuron cells (Schmid \& Maness 2008).

The cell-cell adhesion molecule, which is encoded by the NrCAM gene, is vital for the formation of neurons and their axons, synaptic flexibility, myelination, and highly coordinated function of the brain, such as the brain's cognitive functions of memory and learning (Benson, Schnapp, Shapiro,\& Huntley, 2000). Previous research showed that the disruption of neuronal connection's adhesion during neuronal cell growth in the nervous system may result in neuronal circuit dysfunction and can be the etiological foundation of many neurological disorders (Yang, Hou, Jiang, \& Zhang, 2014). Hargreaves et al (2014) reported that mutations or abnormal expression of NrCAM are likely to cause alteration in synapse formation. Disruption in the NrCAM function can be associated with psychiatric disorders, including SCZ, Autism, Alzheimer, Mathematic learning disability, and drug addiction (Sakurai, 2012). Furthermore, the protein $\mathrm{NrCAM}$ is a stimulator for the division of astrocyte neuronal cells. Glucocorticoid Receptor (GR) pathway contributes to NrCAM's ability to stimulate cell division as NrCAM proteins adhere to neuron cell, intracellular

The NrCAM mRNAs decrease, whereas calreticulin mRNAs and glutamine mRNAs increase. These two genes are active in the Glucocorticoid Receptor (GR) pathway, which is one of the ten signaling pathways identified as a biomarker in the Veripsych kit within the blood serum of drug-Naïve schizophrenic patients (Tomasik, Schwarz, Guest, \& Bahn, 2012; Sabherwal, English Föcking, Cagney, \& Cotter, 2016). It may be postulated that the activity of $\mathrm{NrCAM}$ and the two proteins in question affect each other, and disruption in either of them will disrupt the intracellular chain of events and cause a complication. As a result, the decreased expres- sion of NrCAM through the GR pathway can also disrupt the nervous system's development.

PRODH is one of the critical known genes concerning SCZ. The role of PRODH in the development of SCZ was frequently reported in different populations (Bassett, Marshall, Lionel, Chow, \& Scherer, 2008). SNP rs2238731 is situated in exon 12 of PRODH and was reported as a functional missense mutation (Bender et al., 2005). SNP rs 2238731 is situated in a translated exon. rs2238731 (V427M) affects the function of proline dehydrogenase, the protein which is encoded by PRODH. Bender and Almashanu reported that the SNP V427M leads to a $30 \%-70 \%$ decrease in the Proline Dehydrogenase Enzyme (POX) among the schizophrenic patients (Bender et al., 2005).

The reduction in the proline dehydrogenase enzyme activity contributes to the development of SCZ by affecting the glutamatergic pathway and, in particular, by affecting the NMDA receptors (Coyle, Tsai, \& Goff, 2003; Zinkstok, et al. 2008). In a study carried out on the relationship between polymorphisms in PRODH and brain cortical volumes, only SNP V427M (rs2238731) was found to be significantly associated with the cortical thickness. Cortical thickness in patients with genotype GA was reported to be smaller compared to those carrying genotype GG. However, this finding needs to be confirmed in larger sample sizes (Ota et al., 2014). The frequency of allele $A$ in rs 2238731 is 0.02 in the American population, and it was reported as the predisposing allele for SCZ (Bender, et al., 2005). Ota et al. (2014) suggested that allele $\mathrm{G}$ in rs 2238731 could be a protective allele for SCZ. Despite the role of rs2238731 in the development of SCZ, no significant association was found between rs2238731 and SCZ among Iranian patients.

The frequency of alleles $\mathrm{G}$ and $\mathrm{A}$ in rs2238731 displayed no meaningful difference between the two patients and healthy groups $(\mathrm{P}=0.881)$. Novel variants $\mathrm{c} .562 \mathrm{C}>\mathrm{T}$ and c.7649 $\mathrm{G}>\mathrm{T}$ in ANKK1 and ANK3 respectively, neither schizophrenic nor non-schizophrenic people were carrying this mutated allele. Recently, the c.562 C $>$ T was registered by characteristic rs 89721885408642 in NCBI.

Novel variant c. $7649 \mathrm{G}>\mathrm{T}$ is situated in exon 13 of ANK3. Proteins encoded by the ANK 3 are those connected to cellular integral proteins. In a study conducted in the Norwegian population, the role of ANK3 in susceptibility to $\mathrm{SCZ}$ was reported. They examined the expression of ANK 3 in blood and found that protein ANK3 increased in the blood of those suffering from SCZ. As a result, they suggested that ANK3 mRNA might be one of the diagnostic biomarkers for diagnosing SCZ (Athanasiu et al., 2010). 
ANK3 is associated with other mental disorders such as autism and mental retardation (Bi et al., 2012; Iqbal et al., 2013). Increased expression of ANK3 was previously reported in blood patients with SCZ. It can be regarded as a factor for the formation of psychological disorders such as mental retardation, autism, and SCZ (Iqbal et al., 2013).

\section{Conclusion}

In this study, for the first time, the NrCAM's polymorphisms were interrogated among a sample of Iranian patients in terms of association with SCZ. Our results suggest the association of NrCAM's SNP rs10235968 with schizophrenia disorder in an Iranian sample. NrCAM expression should be evaluated to understand the role of SNP rs10235968 further. The results obtained in this study are different from other similar studies conducted in other populations and indicate genetic diversity in different populations.

The NrCAM's SNPs can play essential roles in the development of schizophrenia through numerous biological pathways. This study's restriction was the small sample size and the low number of polymorphisms that were evaluated in this research. In our investigation, the sample size was too small to come to a decisive conclusion. Hence, further researches are suggested to support the results of this investigation. Besides corroborating the affiliation between NrCAM polymorphisms and SCZ among the Iranian, more polymorphisms should be evaluated.

\section{Ethical Considerations}

\section{Compliance with ethical guidelines}

All ethical principles are considered in this article. The participants were informed about the purpose of the research and its implementation stages. They were also assured about the confidentiality of their information and were free to leave the study whenever they wished, and if desired, the research results would be available to them.

Funding

This research was extracted from the research project (Grant No. 94001), Funded by the University of Tehran Medical Genetics Laboratory.

\section{Authors' contributions}

All authors equally contributed in preparing this article.

\section{Conflict of interest}

The authors declared no conflict of interest.

\section{Acknowledgments}

We appreciate the all patients who have participated in this investigation. We also appreciate Imam Hussein Hospital's Psychiatry ward and the Iranian Society for Supporting Schizophrenic Patients (AHEBA). Also, we appreciate the personnel of the Tehran Medical Genetics Laboratory for their support.

\section{References}

Arab, A. H., \& Elhawary, N. A. (2015). Association between ANKK1 (rs1800497) and LTA (rs909253) genetic variants and risk of Schizophrenia. BioMed Research International, 2015:821827. [DOI:10.1155/2015/821827] [PMID] [PMCID]

Askland, K., Read, C., O'Connell, C., \& Moore, J. H. (2012). Ion channels and schizophrenia: A gene set-based analytic approach to GWAS data for biological hypothesis testing. $\mathrm{Hu}$ man Genetics, 131(3), 373-91. [DOI:10.1007/s00439-011-1082-x] [PMID] [PMCID]

Athanasiu, L., Mattingsdal, M., Kähler, A. K., Brown, A., Gustafsson, O., \& Agartz, I., et al. (2010). Gene variants associated with schizophrenia in a Norwegian genome-wide study are replicated in a large European cohort. Journal of Psychiatric Research, 44(12), 748-53. [DOI:10.1016/j.jpsychires.2010.02.002] [PMID] [PMCID]

Atz, M. E., Rollins, B., \& Vawter, M. P. (2007). NCAM1 association study of bipolar disorder and schizophrenia: Polymorphisms and alternatively spliced isoforms lead to similarities and differences. Psychiatric Genetics, 17(2), 55. [DOI:10.1097/ YPG.0b013e328012d850] [PMID] [PMCID]

Ayalew, M., Le-Niculescu, H., Levey, D. F., Jain, N., Changala B., \& Patel, S. D., et al. (2012). Convergent functional genomics of schizophrenia: from comprehensive understanding to genetic risk prediction. Molecular Psychiatry, 17(9), 887-905. [DOI:10.1038/mp.2012.37] [PMID] [PMCID]

Barbeau, D., Liang, J. J., Robitalille, Y., Quirion, R., \& Srivastava L. K. (1995). Decreased expression of the embryonic form of the neural cell adhesion molecule in schizophrenic brains. Proceedings of the National Academy of Sciences, 92(7), 2785-9. [DOI:10.1073/pnas.92.7.2785] [PMID] [PMCID]

Bassett, A. S., Marshall, C. R., Lionel, A. C., Chow, E. W., \& Scherer, S. W. (2008). Copy number variations and risk for schizophrenia in 22q11. 2 deletion syndrome. Human Molecular Genetics, 17(24), 4045-53. [DOI:10.1093/hmg/ddn307] [PMID] [PMCID]

Bender, H. U., Almashanu, S., Steel, G., Hu, C. A., Lin, W. W., \& Willis, A., et al. (2005). Functional consequences of PRODH missense mutations. The American Journal of Human Genetics, 76(3), 409-20. [DOI:10.1086/428142] [PMID] [PMCID] 
Benson, D. L., Schnapp, L. M., Shapiro, L., \& Huntley, G. W. (2000). Making memories stick: Cell-adhesion molecules in synaptic plasticity. Trends in Cell Biology, 10(11), 473-482. [DOI:10.1016/S0962-8924(00)01838-9]

Bi, C., Wu, J., Jiang, T., Liu, Q., Cai, W., \& Yu, P., et al. (2012). Mutations of ANK3 identified by exome sequencing are associated with autism susceptibility. Human mutation, 33(12), 1635-8. [DOI:10.1002/humu.22174] [PMID]

Chen, L., \& Zhou, S. (2010). "CRASH" ing with the worm: insights into L1CAM functions and mechanisms. Developmental dynamics: An official publication of the American Association of Anatomists, 239(5), 1490-501. [DOI:10.1002/dvdy.22269] [PMID] [PMCID]

Christofolini, D. M., Bellucco, F. T., Ota, V. K., Belangero, S. I., Cernach, M. C., \& Gadelha, A., et al. (2011). Assessment of 22q11. 2 copy number variations in a sample of Brazilian schizophrenia patients. Schizophrenia Research, 132(1), 99-100. [DOI:10.1016/j.schres.2011.07.007] [PMID]

Coyle, J. T., Tsai, G., \& Goff, D. (2003). Converging evidence of NMDA receptor hypofunction in the pathophysiology of schizophrenia. Annals of the New York Academy of Sciences, 1003(1), 318-27. [DOI:10.1196/annals.1300.020] [PMID]

Elangbam, C. S., Qualls Jr, C. W., \& Dahlgren, R. R. (1997). Cell adhesion molecules - update. Veterinary Pathology, 34(1), 6173. [DOI:10.1177/030098589703400113] [PMID]

Galderisi, S., Merlotti, E., \& Mucci, A. (2015). Neurobiological background of negative symptoms. European Archives of Psychiatry and Clinical Neuroscience, 265(7), 543-58. [DOI:10.1007/ s00406-015-0590-4] [PMID]

Hamosh, A., Scott, A. F., Amberger, J. S., Bocchini, C. A., \& McKusick, V. A. (2005). Online Mendelian Inheritance in Man (OMIM), a knowledgebase of human genes and genetic disorders. Nucleic Acids Research, 33(suppl_1), D514-D517. [DOI:10.1093/nar/30.1.52] [PMID] [PMCID]

Hargreaves, A., Anney, R., O'Dushlaine, C., Nicodemus, K. K., Gill, M., Corvin, A., \& Wellcome Trust Case Control Consortium 2. (2014). The one and the many: Effects of the cell adhesion molecule pathway on neuropsychological function in psychosis. Psychological Medicine, 44(10), 2177-87. [DOI:10.1017/S0033291713002663] [PMID] [PMCID]

Honer, W. G., Falkai, P., Young, C., Wang, T., Xie, J., \& Bonner, J., et al. (1997). Cingulate cortex synaptic terminal proteins and neural cell adhesion molecule in schizophrenia. Neuroscience, 78(1), 99-110. [DOI:10.1016/S0306-4522(96)00489-7]

Iqbal, Z., Vandeweyer, G., van der Voet, M., Waryah, A. M., Zahoor, M. Y., \& Besseling, J. A., et al. (2013). Homozygous and heterozygous disruptions of ANK3: At the crossroads of neurodevelopmental and psychiatric disorders. Human Molecular Genetics, 22(10), 1960-70. [DOI:10.1093/hmg/ddt043] [PMID]

Jacquet, H., Raux, G., Thibaut, F., Hecketsweiler, B., Houy, E., \& Demilly, C., et al. (2002). PRODH mutations and hyperprolinemia in a subset of schizophrenic patients. Human Molecular Genetics, 11(19), 2243-9. [DOI:10.1093/hmg/11.19.2243] [PMID]

Jasiewicz, A., Samochowiec, A., Samochowiec, J., Małecka, I., Suchanecka, A., \& Grzywacz, A. (2014). Suicidal behavior and haplotypes of the Dopamine Receptor Gene (DRD2) and ANKK1 gene polymorphisms in patients with alcohol dependence-preliminary report. PLoS One, 9(11), e111798. [DOI:10.1371/journal.pone.0111798] [PMID] [PMCID]

Jia, P., Wang, L., Meltzer, H. Y., \& Zhao, Z. (2010). Common variants conferring risk of schizophrenia: a pathway analysis of GWAS data. Schizophrenia Research, 122(1-3), 38-42. [DOI:10.1016/j.schres.2010.07.001] [PMID] [PMCID]

Kamiguchi, H., \& Lemmon, V. (1997). Neural cell adhesion molecule L1: Signaling pathways and growth cone motility. Journal of Neuroscience Research, 49(1), 1-8. [DOI:10.1002/ (SICI)1097-4547(19970701)49:13.0.CO;2-H]

Kempf, L., Nicodemus, K. K., Kolachana, B., Vakkalanka, R., Verchinski, B. A., \& Egan, M. F., et al. (2008). Functional polymorphisms in PRODH are associated with risk and protection for schizophrenia and fronto-striatal structure and function. PLoS Genet, 4(11), e1000252. [DOI:10.1371/journal.pgen.1000252] [PMID] [PMCID]

Kim, H. J., Kim, H. G., Kim, M. H., Kwack, K. B., Park, J. K, \& Kim, T., et al. (2009). Association between neuronal cell adhesion molecule (NRCAM) single nucleotide polymorphisms and schizophrenia in a Korean population. Psychiatry and Clinical Neurosciences, 63(1), 123-4. [DOI:10.1111/j.14401819.2008.01893.x] [PMID]

Lane, R. P., Chen, X. N., Yamakawa, K., Vielmetter, J., Korenberg, J. R., \& Dreyer, W. J. (1996). Characterization of a highly conserved human homolog to the chicken neural cell surface protein Bravo/Nr-CAM that maps to chromosome band 7q31. Genomics, 35(3), 456-65. [DOI:10.1006/geno.1996.0385] [PMID]

Levinson, D. F., Duan, J., Oh, S., Wang, K., Sanders, A. R., \& Shi, J., et al. (2011). Copy number variants in schizophrenia: confirmation of five previous findings and new evidence for 3q29 microdeletions and VIPR2 duplications. American Journal of Psychiatry, 168(3), 302-16. [DOI:10.1176/appi. ajp.2010.10060876] [PMID] [PMCID]

Little, S. (1995). Amplification $\square$ refractory mutation system (ARMS) analysis of point mutations. Current Protocols in $\mathrm{Hu}$ man Genetics, 7(1), 8-9. [DOI:10.1002/0471142905.hg0908s07] [PMID]

Liu, H., Heath, S. C., Sobin, C., Roos, J. L., Galke, B. L., \& Blundell, M. L., et al. (2002). Genetic variation at the 22q11 PRODH2/ DGCR6 locus presents an unusual pattern and increases susceptibility to schizophrenia. Proceedings of the National Academy of Sciences, 99(6), 3717-22. [DOI:10.1073/pnas.042700699] [PMID] [PMCID]

Nanfack, A. J., Agyingi, L., Noubiap, J. J. N., Ngai, J. N., Colizzi, V., \& Nyambi, P. N. (2015). Use of amplification refractory mutation system PCR assay as a simple and effective tool to detect HIV-1 drug resistance mutations. Journal of Clinical Microbiology, 53(5), 1662-71. [DOI:10.1128/JCM.00114-15] [PMID] [PMCID]

O'Dushlaine, C., Kenny, E., Heron, E., Donohoe, G., Gill, M., \& Morris, D., et al. (2011). Molecular pathways involved in neuronal cell adhesion and membrane scaffolding contribute to schizophrenia and bipolar disorder susceptibility. Molecular Psychiatry, 16(3), 286-92. [DOI:10.1038/mp.2010.7] [PMID]

Ota, V. K., Bellucco, F. T., Gadelha, A., Santoro, M. L., Noto, C., \& Christofolini, D. M., et al. (2014). PRODH polymorphisms, cortical volumes and thickness in schizophrenia. PloS One, 9(2), e87686. [DOI:10.1371/journal.pone.0087686] [PMID] [PMCID] 
Pulver, A. E., Nestadt, G., Goldberg, R., Shprintzen, R. J., Lamacz, M., \& Wolyniec, P. S., et al. (1994). Psychotic illness in patients diagnosed with velo-cardio-facial syndrome and their relatives. Journal of Nervous and Mental Disease, 182(8), 476-8. [DOI:10.1097/00005053-199408000-00010] [PMID]

Rodriguez, S., Gaunt, T. R., \& Day, I. N. (2009). Hardy-Weinberg equilibrium testing of biological ascertainment for Mendelian randomization studies. American Journal of Epidemiology, 169(4), 505-14. [DOI:10.1093/aje/kwn359] [PMID] [PMCID]

Sabherwal, S., English, J. A., Föcking, M., Cagney, G., \& Cotter, D. R. (2016). Blood biomarker discovery in drug-free schizophrenia: the contribution of proteomics and multiplex immunoassays. Expert review of Proteomics, 13(12), 1141-55. [DOI:10. 1080/14789450.2016.1252262] [PMID]

Sakurai, T. (2012). The role of NrCAM in neural development and disorders-beyond a simple glue in the brain. Molecular and Cellular Neuroscience, 49(3), 351-63. [DOI:10.1016/j. mcn.2011.12.002] [PMID]

Sakurai, T., Ramoz, N., Reichert, J. G., Corwin, T. E., Kryzak, L. \& Smith, C. J., et al. (2006). Association analysis of the NrCAM gene in autism and in subsets of families with severe obsessive-compulsive or self-stimulatory behaviors. Psychiatric Genetics, 16(6), 251-7. [DOI:10.1097/01.ypg.0000242196.81891. c9] [PMID]

Schmid, R. S., \& Maness, P. F. (2008). L1 and NCAM adhesion molecules as signaling coreceptors in neuronal migration and process outgrowth. Current Opinion in Neurobiology, 18(3), 245-50. [DOI:10.1016/j.conb.2008.07.015] [PMID] [PMCID]

Shashi, V., Berry, M. N., \& Keshavan, M. S. (2009). Mechanistic approach to understanding psychosis risk in velocardiofacial syndrome. Current Pediatric Reviews, 5(2), 89-104. [DOI:10.217 4/157339609788185703]

Shirzad, H., Beyraghi, N., Ataei, K. M., \& Akbari, M. T. (2017). Family-based whole-exome sequencing for identifying novel variants in consanguineous families with schizophrenia. Iranian Red Crescent Medical Journal, 19(2). [DOI:10.5812/ircmj.35788]

Tomasik, J., Schwarz, E., Guest, P. C., \& Bahn, S. (2012). Blood test for schizophrenia. European Archives of Psychiatry and Clinical Neuroscience, 262(2), 79-83. [DOI:10.1007/s00406-0120354-3] [PMID]

Vawter, M. P., Howard, A. L., Hyde, T. M., Kleinman, J. E., \& Freed, W. J. (1999). Alterations of hippocampal secreted $\mathrm{N}-\mathrm{CAM}$ in bipolar disorder and synaptophysin in schizophrenia. Molecular Psychiatry, 4(5), 467-75. [DOI:10.1038/ sj.mp.4000547] [PMID]

Vawter, M. P. (2000). Dysregulation of the neural cell adhesion molecule and neuropsychiatric disorders. European Journal of Pharmacology, 405(1-3), 385-95. [DOI:10.1016/S00142999(00)00568-9]

Wang, B., Williams, H., Du, J. S., Terrett, J., \& Kenwrick, S. (1998). Alternative splicing of humanNrCAMin neural and nonneural tissues. Molecular and Cellular Neuroscience, 10(5-6), 287-95. [DOI:10.1006/mone.1997.0658]

Wittke-Thompson, J. K., Pluzhnikov, A., \& Cox, N. J. (2005). Rational inferences about departures from Hardy-Weinberg equilibrium. The American Journal of Human Genetics, 76(6), 967-86. [DOI:10.1086/430507] [PMID] [PMCID]
Yang, X., Hou, D., Jiang, W., \& Zhang, C. (2014). Intercellular protein-protein interactions at synapses. Protein $\mathcal{E}$ Cell, 5(6), 420-44. [DOI:10.1007/s13238-014-0054-z] [PMID] [PMCID]

Yoo, B. K., Shim, J. C., Lee, B. D., Kim, C., Chung, Y. I., \& Park, J. M., et al. (2012). Association of the neuronal cell adhesion molecule (NrCAM) gene variants with personality traits and addictive symptoms in methamphetamine use disorder. Psychiatry Investigation, 9(4), 400. [DOI:10.4306/pi.2012.9.4.400] [PMID] [PMCID]

Yu, H., Bi, W., Liu, C., Zhao, Y., Zhang, D., \& Yue, W. (2014) A hypothesis-driven pathway analysis reveals myelin-related pathways that contribute to the risk of schizophrenia and bipolar disorder. Progress in Neuro-Psychopharmacology and Biological Psychiatry, 51, 140-5.[DOI:10.1016/j.pnpbp.2014.01.006] [PMID]

Zhang, Z., Yu, H., Jiang, S., Liao, J., Lu, T., \& Wang, L., et al (2015). Evidence for association of cell adhesion molecules pathway and NLGN1 polymorphisms with schizophrenia in Chinese Han population. PloS One, 10(12), e0144719. [DOI:10.1371/journal.pone.0144719] [PMID] [PMCID]

Zinkstok, J., Schmitz, N., Van Amelsvoort, T., Moeton, M., Baas, F., \& Linszen, D. (2008). Genetic variation in COMT and PRODH is associated with brain anatomy in patients with schizophrenia. Genes, Brain and Behavior, 7(1), 61-9. https:// doi.org/10.1111/j.1601-183X.2007.00326.x

Kay, S. R., Opler, L. A., \& Lindenmayer, J. P. (1988). Reliability and validity of the positive and negative syndrome scale for schizophrenics. Psychiatry Research, 23(1), 99-110. [DOI:10.1016/0165-1781(88)90038-8] 


\title{
Research Paper: The Protective Effect of Kolaviron on Molecular, Cellular, and Behavioral Characterization of Cerebellum in the Rat Model of Demyelinating Diseases
}

\author{
Gabriel Olaiya Omotoso $^{1}$ (D), Leviticus Oghenevurinrin Arietarhire ${ }^{1} \mathbb{D}$, Ileje Inelo Ukwubile ${ }^{1}$ (D), Ismail Temitayo Gbadamosi ${ }^{1}$ (D)
}

1. Department of Anatomy, Faculty of Basic Medical Sciences, College of Health Sciences, University of Ilorin, Ilorin, Nigeria.

\begin{tabular}{|c|c|}
\hline $\begin{array}{l}\text { Use your device to scan } \\
\text { and read the article online }\end{array}$ & Citation: Olaiya Omotoso, G., Oghenevurinrin Arietarhire, L., Inelo Ukwubile, I., \& Temitayo Gbadamosi, I. (2020). The \\
\hline 口p & Protective Effect of Kolaviron on Molecular, Cellular, and Behavioral Characterization of Cerebellum in the Rat Model of \\
\hline Plt & Demyelinating Diseases. Basic and Clinical Neuroscience, 11(5), 609-618. http://dx.doi.org/10.32598/bcn.9.10.300 \\
\hline 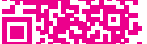 & doi'http://dx.doi.org/10.32598/bcn.9.10.300 \\
\hline
\end{tabular}

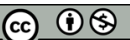

Article info:

Received: 21 Mar 2019

First Revision: 10 Apr 2019

Accepted: 27 Nov 2019

Available Online: 01 Sep 2020

Keywords:

Demyelinating disease, Cerebellum, Cuprizone, Kolaviron, Oxidative stress

\begin{abstract}
A B S T RA C T
Introduction: This study aimed at assessing the protective mechanisms of Kolaviron (KV) on the cerebellum in a rat model of demyelination.

Methods: Twenty-eight male Wistar rats were used in the present study. They were randomly divided into 4 groups of 7 rats. Group A (control) received corn oil $(0.5 \mathrm{~mL} / \mathrm{kg} / \mathrm{d}$ ); group B received $0.2 \%$ Cuprizone (CPZ); group C was treated with $200 \mathrm{mg} / \mathrm{kg} / \mathrm{d}$ of $\mathrm{KV}$, and group D received $0.2 \% \mathrm{CPZ}$ and $200 \mathrm{mg} / \mathrm{kg} / \mathrm{d} \mathrm{KV}$ for 6 weeks. CPZ powder was mixed with the regular diet while $\mathrm{KV}$ was dissolved in corn oil and administered orally. A behavioral test was conducted at the termination of the experiment. Thereafter, the animals were sacrificed and their brains were removed with the excision of the cerebellum. A part of the cerebelli underwent tissue processing with a series of $5 \mu \mathrm{m}$ thick sections cut from paraffin blocks for histological and immunohistochemical assessment. Besides, the remaining cerebellar tissues were homogenized for the spectrophotometric assays of Oxidative Stress (OS) parameters.
\end{abstract}

Results: The current research findings revealed minimal weight gain following CPZ treatment, but significant weight increase in $\mathrm{KV}$-treated rats. $\mathrm{CPZ}$ treatment was associated with a reduction in the number of the line crossed, rearing frequency, rearing duration, center square entry, and center square duration; however, it increased the freezing time, i.e. significantly reversed in the KV-treated animals. Oxidative markers, such as Superoxide Dismutase (SOD) and GPx were reduced in CPZ-treated rats with elevated MDA levels. However, these data were significantly reversed by the co-administration of $\mathrm{CPZ}$ and $\mathrm{KV}$. At the tissue level, the cerebellar cortex was characterized by poorly defined layers, cryptic granules, as well as chromatolysis and pyknotic Purkinje cells with the evidence of hypertrophic astrogliosis.

Conclusion: CPZ treatment significantly depressed locomotor and exploratory activities. Furthermore, it increased OS and cerebellar toxicity. However, KV intervention significantly enhanced behavioral functions and ameliorated CPZ-induced cerebellar degeneration. Moreover, it considerably regulated OS markers in the cerebellum of the rat model of demyelinating diseases.

\footnotetext{
* Corresponding Author:

Gabriel Olaiya Omotoso, PhD.

Address: Department of Anatomy, Faculty of Basic Medical Sciences College of Health Sciences, University of Ilorin, Ilorin, Nigeria.

Tel: +234 (703) 0505707

E-mail: omotoso.go@unilorin.edu.ng; gabrielolaiya@yahoo.com
} 


\section{Highlights}

- Cuprizone (CPZ) treatment depresses locomotor and exploratory activities.

- Kolaviron prevents CPZ-induced behavioral deficit and enhances behavioral functions.

- Kolaviron mitigates cuprizone-induced cerebellar oxidative stress.

\section{Plain Language Summary}

Demyelination is a type of brain disease that involves the shedding away of myelin sheath which eventually leads to loss of axons and neurons in the brain and spinal cord. The cause of this pathological condition ranges from genetic, environment, disease infections to direct injury to the brain. The prevalence of this condition has been on the rise and it is very common among young adults. Currently, there is no cure and the need for finding putative agents for the management, treatment as well as cure is of great urgency. This research was targeted at investigating and establishing a novel compound for management and treatment of this disorder. In this study cuprizone was used to induce demyelination in Wistar rats while Kolaviron, a bioflavonoid complex found in Garcinia kola with anti-oxidative and antiinflammatory properties, was used as a treatment for demyelination. From our results we observed that Kolaviron was able to inhibit the underlying processes involved in demyelination and improved the condition of experimental animals following treatment. This study is important as it portrays and establishes Kolaviron as a potential and good candidate for the management of demyelination as it is cheap and can be made readily available for treating people with various demyelinating conditions worldwide.

\section{Introduction}

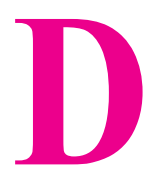

emyelination is a condition that causes the loss of myelin with the relative preservation of axons. It results from diseases that damage myelin sheaths or the myelinforming cells of the Central Nervous System (CNS), oligodendrocytes. The loss of myelin is considered as the cause of numerous CNS conditions (Love, 2006). Demyelination of focal white matter injury can occur in any location of the CNS (Ahmad, Satriotomo, Fazal, Nadeau \& Doré, 2015). The most frequent form of demyelination is observed in Multiple Sclerosis (MS). The volume of white matter lesions in the cerebellum is small, compared to other parts of the CNS; however, the lesions are detrimental to health (Ahmad et al., 2015). Recent insight into the function of the cerebellum indicates that the cerebellum not only controls movement but also impacts motor learning, cognitive behavior, as well as injury-induced sensation and adaptation due to changes in the strength of the connections among its neurons (Houk \& Miller, 2001; Diedrichsen \& Bastian, 2014). The defining clinical manifestation of MS is reported to be associated with cerebellar demyelination (Weinshenker, Issa \& Baskerville, 1996; Rot, Ledinek \& Jazbec, 2008). Despite these interesting findings, there is a paucity of information regarding the molecular, cellular, and behavioral characterization of the cerebellum in de- myelinating diseases. Understanding such characterization could help identify the underlying mechanism involved in demyelination and potential therapeutic targets.

There is a rise in the prevalence of some demyelinating diseases. Accordingly, various animal models have been used to study and find preventive measures or potential cure for these conditions. The cuprizone model, toxin-induced demyelination, has been frequently implemented; it represents a reversible demyelination and remyelination system (Kipp, Clarner, Dang, Copray \& Beyer, 2009; Faizi et al., 2016).

Cuprizone [oxalic acid bis(cyclohexylidenehydrazide)] (CPZ), is a copper chelator that induces demyelination in regions where white matter are located in the rodents' $\mathrm{CNS}$ (Sachs et al., 2014). Its underlying mechanism remains controversial; however, studies have reported that rodents fed with CPZ diet presented megamitochondria, elevated free radicals level due to Oxidative Stress (OS), uncoupling of the oxidative phosphorylation process, oligodendrocyte apoptosis, the disturbance of neurotransmitter homeostasis, synaptic dysfunction, and axonal degeneration (Kipp et al., 2009; Norkute et al., 2009; Hesse et al., 2010; Skripuletz, Gudi, Hackstette \& Stangel., 2011; Tandler \& Hoppel, 1973; Wakabayashi, Asano, \& Kurono, 1975). Although CPZ toxicity targets oligodendrocytes, other macroglia, especially astrocytes could be affected in this respect. 
Astrocytes are specialized glial cells that provide structural and functional support for neurons (Şovrea \& Boşca, 2013). They are involved in synaptogenesis, as well as regulating the communication between already formed synaptic connections (Ota, Zanetti, \& Hallock., 2013). Astrocytes participate in controlling brain homeostasis, and the intrinsic brain defense system (Kettenmann and Verkhratsky); subsequently, they make them respond in case of an insult to the brain.

Contrarily, the treatment and management of complex demyelinating diseases could be achieved through applying the phytochemical constituents of certain plants with medicinal values (Omotoso, Gbadamosi, Afolabi, Abdulwahab \& Akinlolu, 2018). Kolaviron (KV) is among such phytochemicals due to the properties attributed to it (Olaleye and Farombi, 2006; Farombi, Abarikwu, Adedara \& Oyeyemi, 2007). KV is a bioflavonoid complex isolated from the seed of Garcinia kola (bitter kola). The plant is used as an herbal remedy for treating several conditions. It provides antiviral, antibacterial, antifungal, and analgesic properties, and other activities (Olaleye \& Farombi, 2006; Farombi et al., 2007). KV inhibits the excessive production of Nitric Oxide (NO), the expression of cell death regulatory protein, and neuronal cytoskeletal dysregulation (Olajide et al., 2016). The present study aimed to assess the cellular, molecular, and behavioral changes associated with cuprizone toxicity in the cerebellum of Wistar rats, following KV intervention.

\section{Materials and Methods}

Twenty-eight male 9-week-old Wistar rats were obtained from a private animal holding (Tanke, Ilorin). The rats were housed in the Animal House of the Faculty of Basic Medical Sciences, University of Ilorin. They were allowed to acclimatize for 7 days before the onset of the experiment. They were fed on rat chow and water ad libitum. The relevant Ethical approval was obtained from the Ethics Committee of the College of Health Sciences, University of Ilorin, Nigeria. Animal handling and protocols were conducted according to the guidelines of the Ethics Committee. Garcinia kola seeds were procured from a market in Ilorin, Nigeria. Subsequently, they were verified at the herbarium of Botany Department, Faculty of Life Sciences, University of Ilorin (verification code: UILH/001/1217). CPZ was procured from Sigma-Aldrich (Germany), while PhosphateBuffered Solution (PBS; pH 7.0) was freshly prepared. Superoxide Dismutase (SOD), Glutathione Peroxidase (GPx), and Malondialdehyde (MDA) assay kits were also obtained (Abcam ${ }^{\circledR}$, USA). Rats anti-Glial Fibrillary Acidic Protein (GFAP) were acquired from Cell Signaling Technologies, Massachusetts, USA.
The methods employed in the isolation and identification of KV were as earlier described (Farombi, Shrotriya \& Surh, 2009; Olajide et al., 2017). These involved drying Garcinia kola seeds at room temperature, pulverization, extraction, as well as the assessment and confirmation of the purity and identity of the extract (KV) obtained from these processes.

The rats were randomly grouped into 4 classes, labeled as A-D (n=7/group). Group A received $0.5 \mathrm{~mL}$ of Corn Oil (CO) and served as the Control; group $\mathrm{B}$ received $0.2 \% \mathrm{CPZ}$ diet (Praet et al., 2015); group C received $200 \mathrm{mg} / \mathrm{kg}$ bw of KV (Farombi et al., 2009; Omotoso, Olajide, Gbadamosi, Rasheed \& Izuogu, 2018), and, group D received $\mathrm{KV}(200 \mathrm{mg} / \mathrm{kg} \mathrm{bw})$ and CPZ (0.2\%) diet. CO served as the vehicle in which $\mathrm{KV}$ was dissolved for the ease of oral administration. $\mathrm{CPZ}$ diet was constituted to $0.2 \%$ by mixing $0.2 \mathrm{~g} \mathrm{CPZ}$ with a $100 \mathrm{~g}$ standard rat diet. The presented treatment lasted for 42 days.

The open-field test was performed to determine the locomotor activity and exploratory behaviors of rats. Each rat was placed at the center of an open-field box (Yan et al., 2015) and tested for $10 \mathrm{~min}$; in this location, the rats usually have the freedom of movement. The activities of each rat were recorded by a video-camera situated above the area. Following the completion of the exercise, the following data were obtained: the Number of Lines Crossed (NLC), Center Squared Duration (CSD), Center Square Entry (CSE), Freezing Duration (FD), Rearing Frequency $(\mathrm{RF})$, and Rearing Duration (RD).

Sequel to last administration and behavioral study, the rats classified for histological and immunohistochemical evaluation were anesthetized using $20 \mathrm{mg} / \mathrm{kg}$ bw ketamine intraperitoneally; subsequently, they received perfused transcardially with $0.4 \mathrm{M}$ Phosphate-Buffered Saline (PBS), followed by $4 \%$ Paraformaldehyde (PFA). The brain tissues were thereafter excised, each rinsed in $0.25 \mathrm{M}$ sucrose solution thrice for $5 \mathrm{~min}$; then, post-fixed in 4\% PFA for 24 hours. The cerebelli were routinely excised and processed to obtain paraffin wax-embedded blocks. The tissues were stained using Haematoxylin and Eosin and Cresyl fast violet, as described by Fischer, Jacobson, Rose \& Zeller (2008) and Bancroft and Stevens (1982), respectively. The rats used for enzyme studies were sacrificed by cervical dislocation. Their brains were immediately removed and rinsed in $0.25 \mathrm{M}$ sucrose solution thrice for $5 \mathrm{~min}$ each; accordingly, they were placed in $30 \%$ sucrose at $4^{\circ} \mathrm{C}$

The serial sections of the cerebellum were obtained from paraffin blocks and processed as described by Olajide et al. (2017). Immunohistochemistry was performed according 


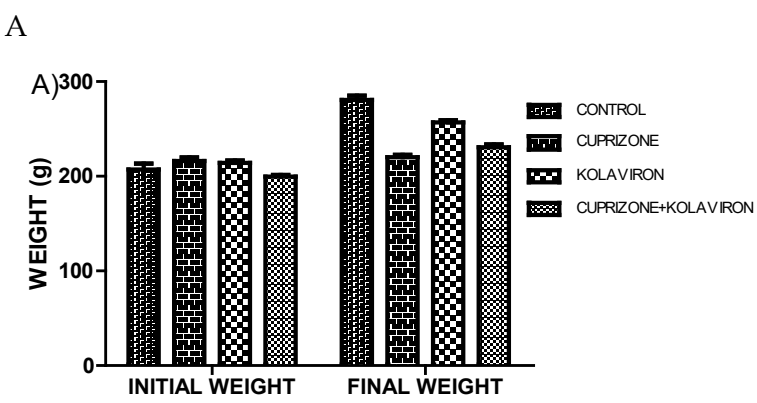

Figure 1. The weights of animals
B

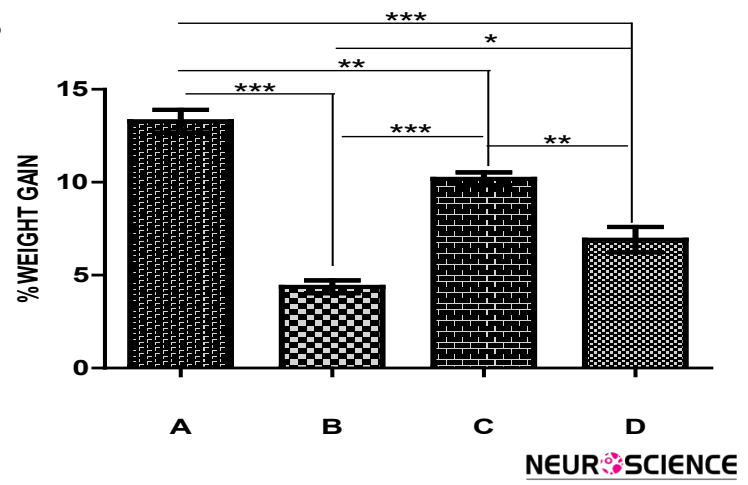

Bodyweight changes across the treated groups: A: Control; B: CPZ; C: KV; and D: CPZ plus KV;

A: Shows the initial and final weights, with all the groups having increased weight differences, though the CPZ group presented very minimal weight differences;

B: The control group (A) had the highest weight gain, while the CPZ-treated group (B) encountered the least weight gain with statistically significant differences, compared to the controls $(\mathrm{P}<0.001)$. Weight gain in the KV-treated group $(\mathrm{C})$ was also high with a statistically significant difference, compared with the controls $(\mathrm{P}<0.01)$; the differences between the KV-treated group and CPZ-treated group was statistically significant $(\mathrm{P}<0.001)$; weight gain in the $\mathrm{CPZ}+\mathrm{KV}$ group $(\mathrm{D})$ was significantly higher than the CPZ-treated group (B) $(\mathrm{P}<0.05)$, but lower than the control group $(\mathrm{A})(\mathrm{P}<0.001)$ and $\mathrm{KV}$-treated group $(\mathrm{C})(\mathrm{P}<0.01)$ Key: ${ }^{*} \mathrm{P}<0.05,{ }^{* *} \mathrm{P}<0.01$, and $\left.{ }^{* *} \mathrm{P}<0.001\right)$.

(A)

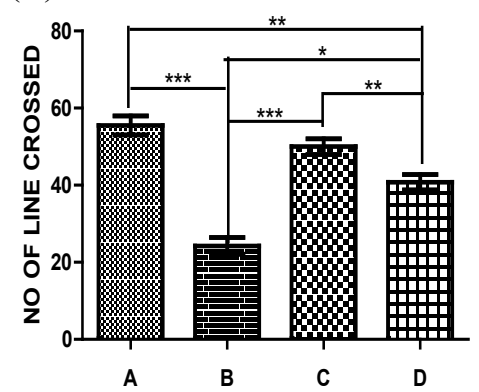

(D)

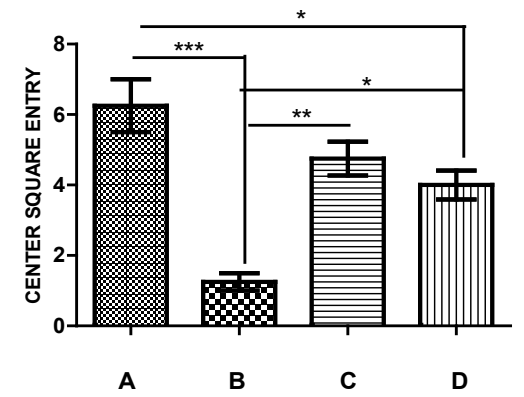

(B)

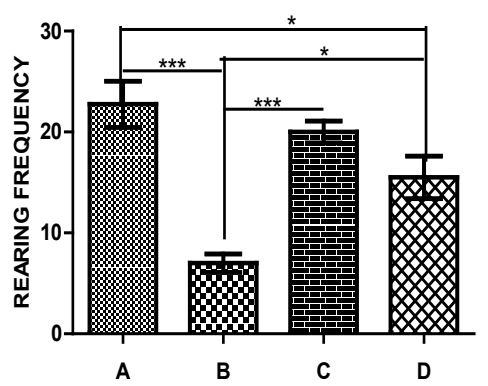

(E)

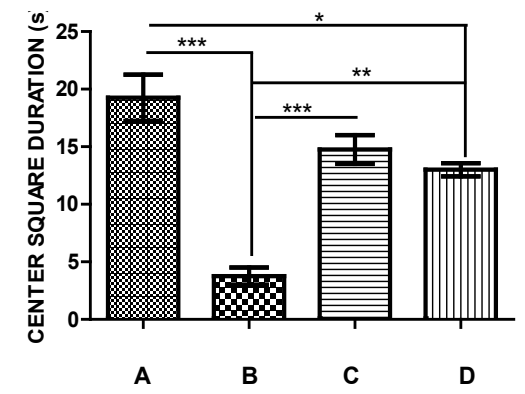

(C)

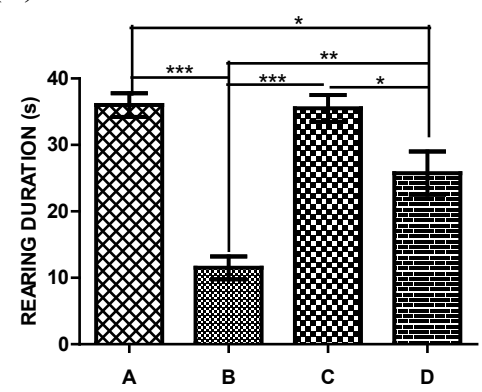

(F)

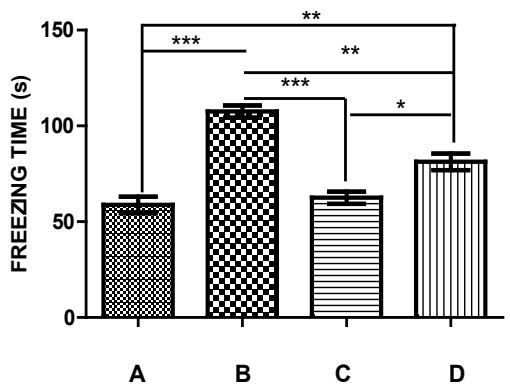

Figure 2. Behavioral tests for locomotion and exploratory activities

The locomotor and exploratory activities of rats across the research groups. A: Control; B: CPZ; C: KV; and D: CPZ and KV. In comparison with $\mathrm{CPZ}$ and $\mathrm{CPZ}+\mathrm{KV}$ treatment groups, animals given $\mathrm{KV}$ treatment experienced an increase in the number of the line crossed (A) $(\mathrm{P}<0.001 \& \mathrm{P}<0.01$, respectively), rearing frequency (B) $(\mathrm{P}<0.001$ : $\mathrm{KV}$ vs. $\mathrm{CPZ}$ groups), rearing duration (C) $(\mathrm{P}<0.001 \& \mathrm{P}<0.05$, respectively), center square entry (D) $(\mathrm{P}<0.01$ : KV vs. $\mathrm{CPZ}$ groups) and center square duration $(\mathrm{E})(\mathrm{P}<0.001$ : $\mathrm{KV}$ vs. CPZ groups). However, there was a decrease in freezing time $(\mathrm{F})$, compared with the groups solely treated with $\mathrm{CPZ}$ and KV $(\mathrm{P}<0.001 \& \mathrm{P}<0.05$, respectively). Animals simultaneously treated with $\mathrm{CPZ}$ and $\mathrm{KV}$ generally demonstrated significantly improved number of the line crossed $(A)(P<0.05)$, rearing frequency $(B)(P<0.05)$, rearing duration $(C)(P<0.01)$, center square entry $(\mathrm{D})(\mathrm{P}<0.05)$, and center square duration $(\mathrm{E})(\mathrm{P}<0.01)$, compared with the $C P Z$ group. 
(A)

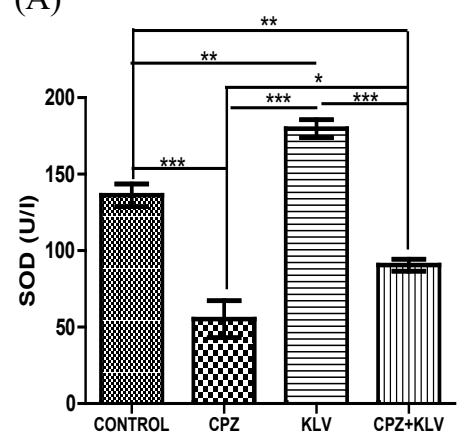

(B)

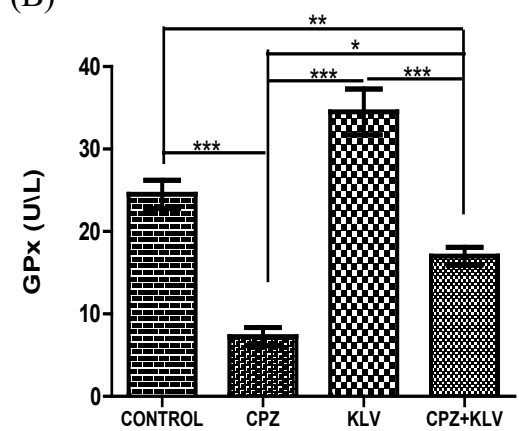

(C)

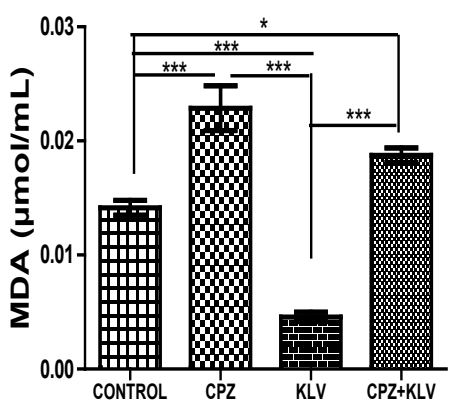

NEUROSCIENCE

Figure 3. The assessment of biochemical oxidative markers (superoxide dismutase, SOD; glutathione peroxidase, GPx \& malondialdehyde, MDA)

A: Shows statistically significant decreases in SOD activity in the CPZ-treated group (B), compared with the controls $(\mathrm{P}<0.001)$, KV-treated group $(\mathrm{C})(\mathrm{P}<0.001)$, and the $\mathrm{CPZ}+\mathrm{KV}$-treated group $(\mathrm{D})(\mathrm{P}<0.05) ; \mathrm{KV}$-treated group had the highest SOD level with statistically significant differences, compared with the control $(\mathrm{P}<0.01)$ and $\mathrm{CPZ}$-treated group $\mathrm{B}(\mathrm{P}<0.001)$; while the $\mathrm{CPZ}+\mathrm{KV}$-treated group $\mathrm{D}$ indicated a significant increase, compared to $\mathrm{CPZ}$-treated group $\mathrm{B}(\mathrm{P}<0.05)$, and a significant decrease, compared to the control $(\mathrm{P}<0.01)$ and $\mathrm{KV}$-treated groups $(\mathrm{P}<0.001)$.

B: Illustrates the activity of GPx across the study groups. GPx activity was least in the CPZ-treated group, compared to the control $(\mathrm{P}<0.001)$, KV-treated $(\mathrm{P}<0.001)$, and $\mathrm{CPZ}+\mathrm{KV}$-treated group $\mathrm{D}(\mathrm{P}<0.05)$. The co-treatment of $\mathrm{CPZ}+\mathrm{KV}$ has led to a significant increase in GPx level, compared with the $\mathrm{CPZ}$ group $(\mathrm{P}<0.05)$; though not reaching the enzyme level in the $\mathrm{KV}$ group $(\mathrm{P}<0.001)$.

C: The level of MDA was significantly elevated in the CPZ group, compared with the control $(\mathrm{P}<0.001)$ and $\mathrm{KV}$ groups $(\mathrm{P}<0.001)$; however, the simultaneous administration of $\mathrm{CPZ}$ and $\mathrm{KV}$ has led to increased MDA levels, compared to the control $(\mathrm{P}<0.05)$ and $\mathrm{KV}$ groups $(\mathrm{P}<0.001)$, which presented the least MDA level. However, the $\mathrm{CPZ}+\mathrm{KV}$ group had a lower level of MDA, compared with the CPZ-treated group, though not statistically significant.

to the method described by Goldstein and Watkins (2008). Moreover, the tissues were stained with anti-Glial Fibrillary Acidic Protein (GFAP) antibody (as a primary antibody). Next, they were treated with biotinylated secondary antibody (goat anti-rabbit) to detect the GFAP-positive cells (astrocytes) in the cerebellum. The mounted slides were viewed with the aid of an Olympus binocular research microscope (Olympus, New Jersey, USA) connected to an Amscope Camera (5.0 MP).

Cerebellar tissue homogenate was prepared with a cold $0.25 \mathrm{M}$ sucrose solution using an automated homogenizer at $4^{\circ} \mathrm{C}$. The tissue homogenate was centrifuged for $10 \mathrm{~min}$ in a microcentrifuge with a centrifugal force of $16099 \times \mathrm{g}$. The supernatants obtained were thereafter aspirated into plain bottles and analyzed for the activities of Superoxide Dismutase (SOD), Glutathione Peroxidase (GPx), and Malondialdehyde (MDA) according to the manufacturer's instruction in the biochemical kits.

All quantitative data were analyzed using GraphPad Prism. The obtained data were presented as the mean and standard error of the mean, using Analysis of Variance (ANOVA) by Tukey's multiple comparisons test. Statistical significance was also considered as $\mathrm{P}<0.05$.

\section{Results}

The feeding pattern among rats treated with $\mathrm{CPZ}$ reduced in the first two weeks of treatment; however, their feeding pattern gradually returned to normal. Additionally, that given $\mathrm{KV}$ experienced an increase in eating habits. However, after the fourth week of KV administration, their feeding habit dropped slightly. The animals treated with both $\mathrm{CPZ}$ and $\mathrm{KV}$ indicated increased feeding patterns during the early stage of administration; however, there was an observable gradual decline in their feeding pattern. Animals treated with CPZ demonstrated no appreciable weight gain (Figure 1A \& B). The group treated with KV and those that concomitantly received $\mathrm{KV}$ and $\mathrm{CPZ}$ revealed a significant weight gain, compared with the group that was treated with CPZ. However, compared with the control group, there was a reduction in their weight (Figure $1 \mathrm{~A} \& \mathrm{~B}$ ).

The open-field test data suggested that demyelinating and degenerative changes observed in CPZ decreased locomotor and exploratory activities $(\mathrm{P}<0.001)$. KV treatment was found to be protective against cuprizoneinduced behavioral deficits; it recorded an alternation in locomotor and exploratory activities, compared to the CPZ group (Figure 2). 


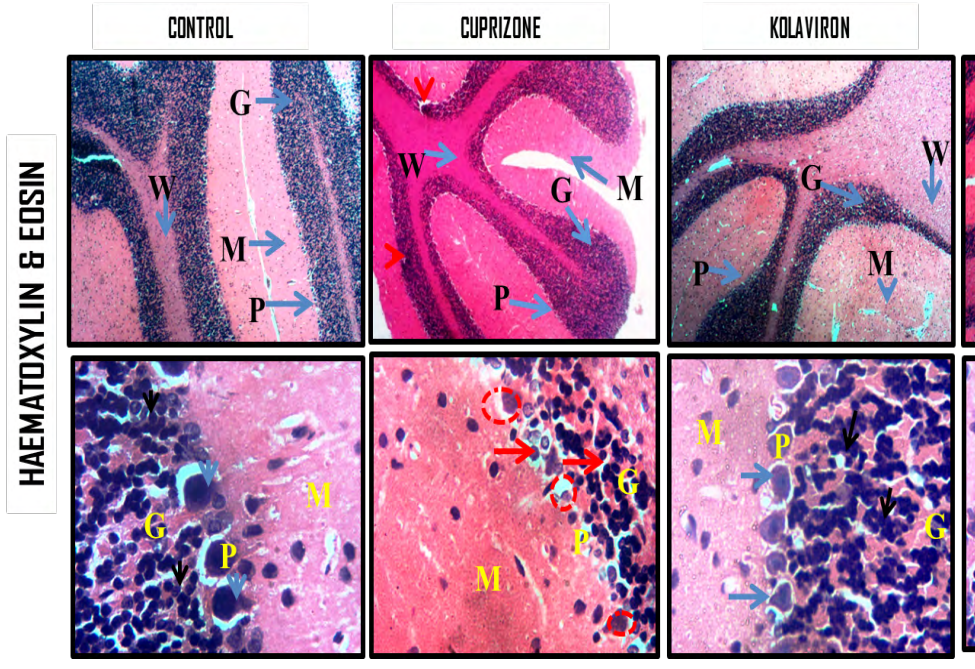

CUPRIZNE + KDLAVIRON

Figure 4. The histological demonstration of the cerebellar cortex of rats

NEUR SCIENCE

Haematoxylin and Eosin (Magnification $\times 40$ and $\times 400$ )

* Olympus binocular research microscope (Olympus, New Jersey, USA).

The representative photomicrographs of the histology of the cerebellar cortices of the studied rats. The different cortical layers were demonstrated as follows: the molecular cell layer (M), Purkinje cell layer (P), granule cell layer (G), and the medullary layer of white matter $(\mathrm{W})$. The structure of the cerebellar cortices of the control and KV-treated rats appeared relatively normal. $\mathrm{CPZ}$ treatment resulted in fragmented granule cell layers and neuropils, and cryptic granule cells, i.e. loosely arranged, degeneration of Purkinje cells and the presence of short dendrites. Neuronal morphology and cerebellar layers in rats co-treated with $\mathrm{KV}$ and CPZ appeared normal with the presence of neurons with healthy somas, axons, and dendrites. (H \& E x40 and x400).

The activities of endogenous oxidative enzymes were assessed to illustrate their involvement in CPZ-induced demyelination and understand the mechanistic inhibitory roles of KV. The spectrophotometric assay data of the SOD profile from cerebellar homogenates presented a normal SOD level in the cerebellum of the control and $\mathrm{KV}$ groups; these data were associated with a significant increase in group $\mathrm{C}$. The SOD level significantly reduced in rats intoxicated with $\mathrm{CPZ}$, compared with other groups. However, rats that concomitantly received CPZ and $\mathrm{KV}$ revealed a significant increase in SOD level, compared to the group that received CPZ (Figure 3A). The GPx level of cerebellar lysates was examined (Figure 3B). The related findings revealed that the control and KV-treated rats presented elevated GPx levels, compared to those treated with $\mathrm{CPZ}$ and adjunct $\mathrm{CPZ}$ and $\mathrm{KV}$. Furthermore, animals co-treated with $\mathrm{CPZ}$ and $\mathrm{KV}$ recorded a higher GPx level, compared with the CPZtreated rats. MDA level was also assessed to determine the degree of lipid peroxidation in cerebellar lysates in the research groups. The relevant results (Figure 3C) suggested that $\mathrm{KV}$-treated rats experienced decreased expression of MDA in the cerebellar homogenate. The CPZ-treated had increased MDA level, compared to the other groups. The rats treated concomitantly with $\mathrm{KV}$ and $\mathrm{CPZ}$ demonstrated a significant downregulation of MDA expression, compared to the CPZ-treated group.

The histochemistry of the cerebellum was demonstrated using Haematoxylin and Eosin (H \& E) and Cresyl Fast Violet (CFV) staining methods. Figure 4 shows the panoramic view and high power magnification of the microarchitecture of cerebellar cortices with distinct cell types and cell layers. The Control and KV-treated rats presented well-arranged cerebellar layers with obvious soma and dendrites; they project deep into the molecular layers, having a fan-like shape nucleus with well-stained white matter regions. Furthermore, the granular cell layers in the groups were comprised of well-arranged small granule cells. Neuronal morphology of rats intoxicated with CPZ demonstrated fragmented cerebellar layers with cryptic granules, degenerating Purkinje cells with pyknotic cell bodies, and short dendritic processes. However, the rats treated with both $\mathrm{CPZ}$ and $\mathrm{KV}$ indicated cerebellar layers and neuronal morphology, i.e. similar to that of the controls.

GFAP immunohistochemistry method was used to demonstrate astrocytic morphology and distribution in the cerebellar cortex of the explored Wistar rats. GFAP immunopositive cells within the cerebellar cortex of 


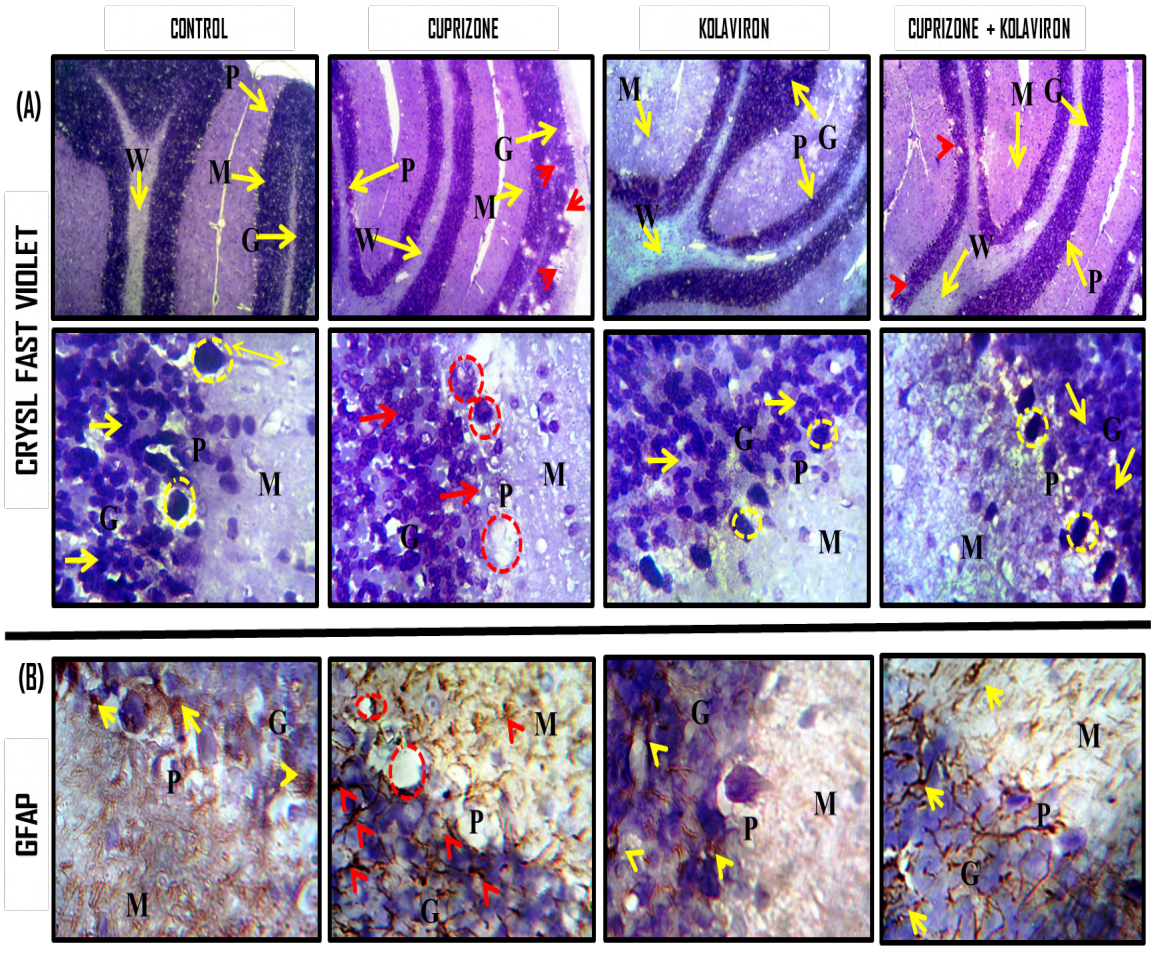

NEUR:SCIENCE

Figure 5. The histochemical demonstration of the cerebellar cortex and Immunohistochemical expression of astrocytes

A- Cresyl fast violet ((Magnification $\times 40$ and $\times 400)$; B: GFAP= glial fibrillary acidic protein immunohistochemical staining with anti-GFAP primary antibody (Magnification $\times 400)$

* Olympus binocular research microscope (Olympus, New Jersey, USA).

A \& B: Representative photomicrographs presenting the Nissl profile (A) and immunohistochemical demonstration of astrocytes (B) using CFV and anti-GFAP, respectively. Fig. 5A shows the panoramic view and high-power magnification of the cerebellar cortex stained with CFV across the research groups. The rats treated with either $\mathrm{CO}(\mathrm{A})$ or $\mathrm{KV}(\mathrm{C})$ revealed well-stained intensity and cellular density within the granular layer and white matter layer with deeply stained and well expressed Purkinje cells (yellow dotted circles), and granule cells (yellow arrows). Rats fed with CPZ (B) presented poorly stained intensity and cellular density within the granular layer with chromatolysis Purkinje cells (red dotted circles) and granule cells (red arrows). However, the studied rats treated with combined CPZ and KV experienced improved staining intensity and cellular density; also deeply stained and well expressed Purkinje cells (yellow dotted circles), and granule cells (yellow arrows). GFAP immunopositive cells;

B: (black arrows) appeared scanty around neurons in the control and KV-treated groups with relatively normal architecture. The CPZ-treated rats demonstrated evidence of increased astrocytic densities with reactive astroglia within the granule cell layer and hypertrophic cells. Meanwhile, the rats co-treated with $\mathrm{CPZ}$ and KV indicated close similarities with those of the control and KV-treated groups. (magnification $\mathrm{CFV}=\mathrm{x} 40$ and $\mathrm{x} 400$; GFAP $=x 400$ ).

the control and $\mathrm{KV}$-treated rats appeared scanty around neurons; they also were observable in between layers, with regular processes, distribution, and sizes within the neuropil. However, increased astrocytic densities with reactive astroglia within the granule cell layer and hypertrophic cells appeared within the cerebellar layers in CPZ-treated rats. Expressing astrocytes within the cerebellar cortex of rats treated with Kv and CPZ had close similarities with the control and KV-treated groups. Astrocytic processes, cellular distribution, and size were normal in the control and KV-treated rats.

\section{Discussion}

Demyelination of focal white matter injury can occur in any location of the CNS (Ahmad, Satriotomo, Fazal, Nadeau \& Doré, 2015). The most frequent form of demyelination is observed in Multiple Sclerosis (MS) Eating and diets are essential factors in controlling body weight (Drapeau et al., 2004). Studies reported reduced feeding habits during the first 2 weeks of $\mathrm{CPZ}$ consumption and gradual improvement in feeding habits post CPZ diet withdrawal (Sachs et al., 2014; Praet et al., 2015; Steel- 
man, Thompson \& Li, 2012). Therefore, it is suggested that the observed bodyweight reduction in this study was caused by reduced feeding pattern and the disruption of healthy energy flow. KV treatment significantly prevented CPZ-associated weight loss; i.e. probably caused by its ability to prevent normal energy disruption owing to its potential to mop up excess free radical. This might be the reason for the detected disruption.

As a correlative test for cellular and neuropathological changes in the cerebellar cortex in this study, the exploratory activities of rats were assessed using the open field test (Gould, Dao, \& Kovacsics, 2010).

Our results revealed a significant reduction in exploratory activities in CPZ-treated rats, corroborating a previous study by Faizi et al. (2016). However, KV significantly countered these effects. Thus, locomotor and exploratory activities depend on the intact integrity of the myelin sheath. Prior research suggested increased exploratory activities of rats following $\mathrm{KV}$ treatment after NaN3-induced neurodegeneration (Olajide et al., 2016).

Decreased SOD and GPx levels, as observed in the current study, has been implicated in the mechanism through which CPZ induces demyelination (Biancotti, Kumar \& de Vellis, 2008; Witherick, Wilkins, Scolding, \& Kemp, 2010; Praet et al., 2014). Faizi et al. (2016) reported a decrease in the SOD level following the administration of $\mathrm{CPZ}$ due to the reduced level of $\mathrm{Cu}++$, i.e. required for the proper functioning of SOD. KV treatment improved these antioxidants' levels within the cerebellar cortex of rats. SOD catalyzes the dismutation of superoxide (O2-) radical (Sun and Trumpower, 2003; Hayyan et al., 2016); if upregulated by KV, it could prevent the cytotoxic effects of $\mathrm{O} 2-$ molecules. Thus, it prevents oxidative-related damage. GPx is an enzyme capable of detoxifying Reactive Oxygen Species (ROS) and nucleophilic compounds. They could initiate lipid hydroperoxides to their corresponding alcohols and water, respectively (Muller et al., 2007). Lipid peroxidation and OS following CPZ treatment have been previously documented (Xuan et al., 2015). However, treating rats with KV significantly reduced the MDA level. Such data suggest that KV could inhibit lipid peroxidation due to its antioxidant properties.

CPZ-induced cerebellar injury revealed various degrees of structural damage to the cellular components and the normal architectural pattern of the cerebellum. These alterations in the cellular morphology of the cerebellum following CPZ treatment adversely affect signal processing. It also impacts the functionality of the syn- aptic complex, as the common features in patients with demyelinating diseases (Kutzelnigg et al., 2007; Rot et al., 2008).

Furthermore, CPZ intoxication induces megamitochondria and OS, leading to energy flow disruption and shortage (Tandler and Hoppel, 1973). The energy flow disruption and depletion alter the proper functioning of the endoplasmic reticulum, i.e. important in neuronal protein synthesis. This often causes the cellular degeneration and disintegration of oligodendrocytes perikaryon and myelin sheath (Praet et al., 2014). The neurotoxic nature of $\mathrm{CPZ}$ may explain the mechanisms by which it induces cellular degeneration and white matter demyelination. Interestingly, rats were given KV concomitantly reflected cerebellar morphology, i.e. similar to the controls. The ability of KV to restore the chromogenic nature of the Nissl substance following CPZ-induced damage might be due to its ability to prevent ER stress; this process inhibits the pathways that lead to failure of protein production owing to its antioxidative property and ROS scavenging ability.

Studies have revealed an increase in astrocytic activity following one-week CPZ intoxication (Zatta et al., 2005). The activation of astrocytes could result in extensive astrogliosis which may persist through the period of remyelination (Zaaraoui et al., 2008; Gudi et al., 2009). In the current work, changes observed on the cerebellum were characteristic of reactive astrogliosis. Accordingly, these were however absent in the cerebellar cortex of rats treated with KV. This finding suggests a cytoprotective role of $\mathrm{KV}$.

\section{Conclusion}

$\mathrm{KV}$ exhibited neuroprotection against the behavioral deficit, OS, astrogliosis, demyelination, and CPZ-induced cortical neuronal damage in adult male Wistar rats. These findings provide a lead on the potentials of $\mathrm{KV}$, as a neuroprotective agent against demyelinating diseases.

\section{Ethical Considerations}

\section{Compliance with ethical guidelines}

The Study was approved by the Ethical Review Committee, University of Ilorin (UERC/ASN/2017/969). 
Funding

This research did not receive any grant from funding agencies in the public, commercial, or non-profit sectors.

\section{Conflict of interest}

The authors declared no conflicts of interest.

\section{Acknowledgments}

The authors acknowledge the assistance and guidance of Dr. O.J. Olajide, affiliated with the Department of Anatomy, University of Ilorin.

\section{Reference}

Ahmad, A. S., Satriotomo, I., Fazal, J., Nadeau, S. E., \& Doré, S (2015). Considerations for the optimization of induced white matter injury preclinical models. Frontiers in Neurology, 6, 172. [DOI:10.3389/fneur.2015.00172] [PMID] [PMCID]

Bancroft, J.D., Stevens, A. (1982). Theory and Practice of Histological Techniques, $2^{\text {ed }}$. Edinburgh: Churchill Livingstone. https://books.google.com/books?

Biancotti, J.C., Kumar, S., de Vellis, J. (2008). Activation of inflammatory response by acombination of growth factors in cuprizone-induced demyelinated brain leadsto myelin repair. Neurochemical Research, 33(12), 2615-28. [DOI:10.1007/s11064008-9792-8] [PMID]

Diedrichsen, J., Bastian, A.J. (2014). Cerebellar function. In: M.S Gazzaniga. (Ed.), The Cognitive Neuroscience, fifth ed. Cambridge: MIT Press.

Drapeau, V., Després, J. P., Bouchard, C., Allard, L., Fournier, G., Leblanc, C., et al. (2004). Modifications in food-group consumption are related to long-term body-weight changes. Journal of Clinical Nutrition, 80(1), 29-37. [DOI:10.1093/ ajcn/80.1.29] [PMID]

Faizi, M., Salimi, A, Seydi, E., Naserzadeh, P., Kouhnavard, M. Rahimi, A., et al. (2016). Toxicity of cuprizone a Cu2p chelating agent on isolated mouse brain mitochondria: A justification for demyelination and subsequent behavioral dysfunction. Toxicology Mechanisms and Methods, 26(4), 276-83. [DOI:1 0.3109/15376516.2016.1172284] [PMID]

Farombi, E. O., Abarikwu, S. O., Adedara, I. A., Oyeyemi, M.O. (2007). Curcumin and kolaviron ameliorate di-n-butylphthalate-induced testicular damage in rats. Basic $\mathcal{E}$ Clinical Pharmacology \& Toxicology, 100(1), 43-8. [DOI:10.1111/j.17427843.2007.00005.x] [PMID]

Farombi, E.O., Shrotriya, S., Surh, Y. (2009). Kolaviron inhibits dimethyl nitrosamine-induced liver injury by suppressing cox-2 and iNOS expression via NF-ÊB and AP-1. Life Sciences 84, 149-55. [DOI:10.1016/j.lfs.2008.11.012] [PMID]
Fischer, A.H., Jacobson, K., Rose, J., Zeller, R. (2008). Hematoxylin and eosin staining of tissue and cell sections. CSH protocols, 2008, pdb.prot 4986. [DOI.:10.1101/pdb.prot4986]

Goldstein, M., Watkins, S. (2008). Immunohistochemistry. In M.A. Frederick, (Ed.), Current Protocols In Molecular Biology, (pp. 44-56), East Anglia: Wiley and Sons.

Gould, T. D., Dao, D. T., Kovacsics, C. E. (2010). The open field test. Mood and Anxiety Related Phenotypes in Mice, 42, 1-20. [DOI:10.1007/978-1-60761-303-9_1]

Gudi, V., Gingele, S., Skripuletz, T., Stangel, M (2014). Glial response during cuprizone-induced de- and remyelination in the CNS: Lessons learned. Frontiers in Cellular Neuroscience, 8 73. [DOI:10.3389/fncel.2014.00073] [PMID] [PMCID]

Gudi, V., Moharregh-Khiabani, D., Skripuletz, T., Koutsoudaki, P.N., Kotsiari, A., Skul-jec, J. (2009). Regional differences between grey and white matter in cuprizone induced demyelination. Brain Research, 1283, 127-38. [DOI:10.1016/j.brainres.2009.06.005] [PMID]

Hayyan, M., Hashim, M. A., Al-Nashef, I. M. (2016). Superoxide Ion: generation and chemical implications. Chemical Reviews, 116(5), 3029-85. [DOI:10.1021/acs.chemrev.5b00407] [PMID]

Hesse, A., Wagner, M., Held, J., Bruck, W., Salinas-Riester, G., Hao, Z. (2010). Intoxic demyelination oligodendroglial cell death occurs early and is FAS independent. Neurobiology of Disease, 37(2), 362-9. [DOI:10.1016/j.nbd.2009.10.016] [PMID] [PMCID]

Houk, J.C., Miller, L.E. (2001). Cerebellum: Movement regulation and cognitive functions. In: Encyclopedia of Life Science. Nature Publishing Group. [DOI:10.1038/npg.els.0000036]

Kettenmann, H., Verkhratsky, A. (2011). Neuroglia - living nerve glue. Fortschr Neurol Psychiatr, 79(10), 588-97. [DOI:10.1055/s-0031-1281704] [PMID]

Kipp, M., Clarner, T., Dang, J., Copray, S., Beyer, C. (2009). The cuprizone animal model: New insights into an old story. Acta Neuropathologica, 118, 723-736. [DOI:10.1007/s00401-009-05913] [PMID]

Kutzelnigg, A., Faber-Rod, J. C., Bauer, J., Lucchinetti, C. F., Sorensen, P. S., Laursen, H. (2007). Widespread demyelination in the cerebellar cortex in multiple sclerosis. Brain Pathology 17(1), 38-44. [DOI:10.1111/j.1750-3639.2006.00041.x] [PMID]

Love, S. (2006). My approach, demyelinating diseases. Journal of Clinical Pathology, 59, 1151-9. [DOI:10.1136/jcp.2005.031195] [PMID] [PMCID]

Muller, F. L., Lustgarten, M. S., Jang, Y., Richardson, A., van Remmen, H. (2007). Trends in oxidative aging theories. Free Radical Biology and Medicine, 43(4), 477-503. [DOI:10.1016/j. freeradbiomed.2007.03.034] [PMID]

Norkute, A., Hieble, A., Braun, A., Johann, S., Clarner, T., Baumgartner, W. (2009). Cuprizone treatment induces demyelination and astrocytosis in the mouse hippocampus. Journal of Neuroscience Research, 87(6), 1343-55. [DOI:10.1002/jnr.21946] [PMID]

Olajide, O. J., Enaibe, B. U., Bankole, O. O., Akinola, O. B., Laoye, B. J., Ogundele, O. M. (2016). Kolaviron was protective agains sodium azide (NaN3)-induced oxidative stress in the prefrontal cortex. Metabolic Brain Disease, 31(1), 25-35. [DOI:10.1007/ s11011-015-9674-0] [PMID] 
Olajide, O. J., Ugbosanmi, A. T., Enaibe, B. U., Ogunrinola, K. Y., Lewu, S.F., Asogwa, N. T., et al. (2017). Cerebellar molecular and cellular characterization in rat models of Alzheimer's disease: Neuroprotective mechanisms of Garcinia biflavonoid complex.

Annals of Neurosciences, 24(1), 32-45. [DOI:10.1159/000464421] [PMID] [PMCID]

Olaleye, S. B., \& Farombi, E. O. (2006). Attenuation of indomethacin- and $\mathrm{HCl} /$ ethanol-induced oxidative gastric mucosa damage in rats by kolaviron, a natural biflavonoid of Garcinia kola seed. Phytotherapy Research, 20, 14-20. [DOI:10.1002/ ptr.1793] [PMID]

Omotoso, G. O., Gbadamosi, I. T., Afolabi, T. T., Abdulwahab A. B., Akinlolu, A. A. (2018). Ameliorative effects of Moringa on cuprizone-induced memory decline in rat model of multiple sclerosis. Anatomy and Cell Biology, 51, 119-27. [DOI:10.5115/ acb.2018.51.2.119] [PMID] [PMCID]

Omotoso, G. O., Olajide, O. J., Gbadamosi, I. T., Rasheed, M. A., Izuogu, C. T. (2018). Kolaviron protects the prefrontal cortex and hippocampus against histomorphological and neurobehavioral changes in cuprizone model of multiple sclerosis. Malaysian Journal of Medical Sciences, 25(2), 50-63. [DOI:10.21315/mims2018.25.2.6] [PMID] [PMCID]

Ota, Y., Zanetti, A. T., \& Hallock, R. M. (2013). The role of astrocytes in the regulation of synaptic plasticity and memory formation. Neural Plasticity, 2013. [DOI:10.1155/2013/185463] [PMID] [PMCID]

Praet, J., Guglielmetti, C., Berneman, Z., van der Linden, A., Ponsaerts, P. (2014). Cellular and molecular neuropathology of the cuprizone mouse model: Clinical relevance for multiple sclerosis. Neuroscience \& Biobehavioral Reviews, 47, 485-505. [DOI:10.1016/j.neubiorev.2014.10.004] [PMID]

Praet, J., Orije, J., Kara, F., Guglietmetti, C., Santermans, E. Daans, J., et al. (2015). Cuprizone-induced demyelination and demyelination-associated inflammation result in different proton magnetic resonance metabolite spectra. NMR in Biomedicine, 28(4), 505-513. [DOI:10.1002/nbm.3277] [PMID] [PMCID]

Rot, U., Ledinek, A. H., \& Jazbec, S. Š. (2008). Clinical, magnetic resonance imaging, cerebrospinal fluid and electrophysiological characteristics of the earliest multiple sclerosis. Clinical Neurology and Neurosurgery, 110(3), 233-8. [DOI:10.1016/j. clineuro.2007.11.001] [PMID]

Sachs, H. H., Bercury, K. K., Popescu, D. C., Narayanan, S. P., \& Macklin, W. B. (2014). A new model of cuprizonemediated demyelination/remyelination. ASN Neuro, 6(5), 1759091414551955. [DOI:10.1177/1759091414551955] [PMID] [PMCID]

Skripuletz, T., Gudi, V., Hackstette, D., \& Stangel, M. (2011). De-and remyelination in the CNS white and grey matter induced by cuprizone: The old, the new, and the unexpected. Histology and Histopathology, 26(12), 1585-97. https://digitum.um.es/digitum/bitstream/10201/49540/1/Skripuletz-26-1585-1597-2011.pdf

Sovrea, A. S., \& Boşca, A. B. (2013). Astrocytes reassessment - an evolving concept part one: Embryology, biology, morphology and reactivity. Journal of Molecular Psychiatry, 1(1), 18 [DOI:10.1186/2049-9256-1-18] [PMID] [PMCID]
Steelman, A., Thompson, J. P., Li, J. (2012). Demyelination and remyelination in anatomically distinct regions of the corpus callosum following cuprizone intoxication. Neuroscience Research, 72(1), 32-42. [DOI:10.1016/j.neures.2011.10.002] [PMID] [PMCID]

Sun, J., \& Trumpower, B. L. (2003). Superoxide anion generation by the cytochrome bc1 complex. Archives of Biochemistry and Biophysics, 419(2), 198-206. [DOI:10.1016/j.abb.2003.08.028] [PMID]

Tandler, B., \& Hoppel, C. L. (1973). Division of giant mitochondria during recovery from cuprizone intoxication. The Journal of Cell Biology, 56(1), 266-72. [DOI:10.1083/jcb.56.1.266] [PMID] [PMCID]

Wakabayashi, T., Asano, M., \& Kurono, C. (1975). Mechanism of the formation of megamitochondria induced by copperchelating agents, II; Isolation and some properties of megamitochondria from the cuprizone-treated mouse liver. Pathology International, 25(1), 39-49. [DOI:10.1111/j.1440-1827.1975. tb00148.x]

Weinshenker, B. G., Issa, M., \& Baskerville, J. (1996). Long-term and short-term outcome of multiple sclerosis: A 3-year followup study. Archives of Neurology, 53(4), 353-358. [DOI:10.1001/ archneur.1996.00550040093018] [PMID]

Witherick, J., Wilkins, A., Scolding, N., \& Kemp, K. (2010). Mechanisms of oxidative damage in multiple sclerosis and a cell therapy approach to treatment. Autoimmune diseases, 2011. [DOI:10.4061/2011/164608] [PMID] [PMCID]

Xuan, Y., Yan, G., Wu, R., Huang, Q., Li, X., \& Xu, H. (2015). The cuprizone-induced changes in 1H-MRS metabolites and oxidative parameters in C57BL/ 6 mouse brain: Effects of quetiapine. Neurochemistry International, 90, 185-92. [DOI:10.1016/j. neuint.2015.08.015] [PMID]

Yan, G., Xuan, Y., Dai, Z., Shen, Z., Zhang, G., Xu, H., \& Wu, R. (2015). Brain metabolite changes in subcortical regions after exposure to cuprizone for 6 weeks: Potential implications for schizophrenia. Neurochemical Research, 40(1), 49-58. [DOI:10.1007/s11064-014-1464-2] [PMID]

Zaaraoui, W., Deloire, M., Merle, M., Girard, C., Raffard, G., Biran, M., ... \& Franconi, J. M. (2008). Monitoring demyelination and remyelination by magnetization transfer imaging in the mouse brain at 9.4 T. Magnetic Resonance Materials in Physics, Biology and Medicine, 21(5), 357-62. [DOI:10.1007/s10334-0080141-3] [PMID] [PMCID]

Zatta, P., Raso, M., Zambenedetti, P., Wittkowski, W., Messori, L., \& Piccioli, F., et al. (2005). Copper and zinc dismetabolism in the mouse brain upon chronic cuprizone treatment. Cellular and Molecular Life Sciences CMLS, 62(13), 1502-13. [DOI:10.1007/s00018-005-5073-8] [PMID] 


\section{Research Paper: Diagnostic Challenges and Prospects Associated With Zoonotic Tuberculosis of Central Nervous System}

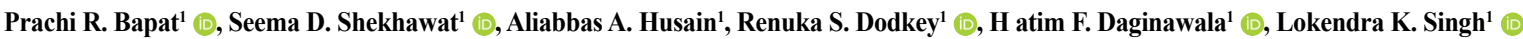
Rajpal S. Kashyap ${ }^{1 *}$ Challenges and Prospects Associated With Zoonotic Tuberculosis of Central Nervous System. Basic and Clinical Neuroscience, 11(5), 619-630. http://dx.doi.org/10.32598/bcn.11.5.10.803.3

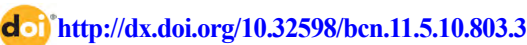

\section{(c) (i) (\$)}

\section{Article info:}

Received: 29 Nov 2019

First Revision: 10 Dec 2019

Accepted: 11 Mar 2020

Available Online: 01 Sep 2020

Keywords:

Duplex PCR, Mycobacterium Bovis, Tuberculous meningitis, Zoonoses

\section{A B S T RA C T}

Introduction: The diagnosis of Tuberculous Meningitis (TBM) has remained a challenge due to its insidious onset and the failure of conventional diagnostic tests. The present study aimed to identify the mycobacterial pathogen in the CSF of patients with TBM and a poor prognosis.

Methods: We retrospectively recruited $224 \mathrm{TBM}$ and 34 non-TBM patients admitted to the Central India Institute of Medical Sciences, Nagpur, India, in 2014. The CSF samples of these patients were subjected to a duplex PCR assay for the species-specific identification of the causative pathogen.

Results: M. bovis and infection with M.tuberculosis were detected in 7\% (18) and 32.9\% (85) of the patients, respectively. Moreover, $14 \%$ (36) of the study samples were culture positive; however, the mycobacterial pathogens could not be differentiated to the species level.

Conclusion: The present study findings emphasized the potentially vital importance of $\mathrm{M}$. bovis identification for appropriate patient management. The obtained data also demonstrated the persistent significance of M. bovis, as a zoonotic pathogen. 


\section{Highlights}

- The study aimed at identification of M. bovis infection in CSF of TBM cases.

- Incidence of M. bovis and MTB by duplex PCR assay was 7\% \& 32.9 respectively

- The study highlights M. bovis as major zoonotic pathogen in TBM cases

- Results advocates diagnosis of M. bovis in TBM cases with poor treatment outcomes

\section{Plain Language Summary}

Tuberculosis caused by M. bovis is important yet neglected zoonotic disease of public health importance. Central nervous infection (CNS) caused by M. bovis is often associated with insidious onset due to lack of specific diagnostic tools and its intrinsic resistant to frontline TB drug pyrazinamide. Lack of specific identification \& differentiation of etiological agent in Tuberculous meningitis (TBM) infection often leads to poor treatment outcomes, high rates of neurological morbidity \& possibility of development of drug resistance. In current study we, investigated utility of inhouse designed duplex PCR assay for simultaneous diagnosis of M.tuberculosis (MTB) and M. bovis in CSF samples of TBM cases associated with poor treatment outcomes. The incidence of M. bovis by duplex PCR assay was found to be 7\% underlining its importance as important zoonotic pathogen associated with TBM infection. The study advocates utility of such molecular assay for diagnosis of M. bovis pathogen in TBM infection to improve efficacy of treatment outcome \& reduce chances of drug resistance.

\section{Introduction}

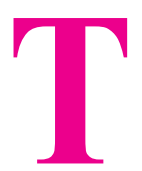

he last decade has witnessed shifting trends in Tuberculosis (TB) infection, with Extra Pulmonary Tuberculosis (EPTB) emerging as an essential entity (Jain, 2011). The TB of the Central Nervous System (CNS) is the most fatal extra-pulmonary disease that affects approximately $10 \%$ of the population globally affected with TB. The estimated mortality due to Tuberculous Meningitis (TBM) in India is 1.5 per 100000 individuals (Murthy, 2010; Kaur, et al., 2015). The diagnosis of TBM has remained a challenge to the clinicians. This is due to nonspecific clinical manifestation, which varies widely; thus, creating a major obstacle in the initiation of the treatment. Moreover, the limited sensitivity of conventional diagnostic tests (smear microscopy $\&$ culture) due to the paucibacillary nature of samples also contribute to a delayed diagnosis (Jain, 2011; Purohit \& Mustafa, 2015).

Different studies have reported the possible association between variables, such as age, the stage of the disease, clinical characteristics, mycobacterial isolation from $\mathrm{Ce}$ rebrospinal Fluid (CSF), etc., and the manifestation of TBM. The reason for poor prognosis has been ascertained to the lack of appropriate diagnostic tools for identifying the causative organism (Kaur, et al., 2015; Ahmadinejad,
Ziaee, Aghsaeifar, \& Reiskarami, 2003). Despite being a TB endemic country, limited preventive measures have been taken to improve the subsequent outcomes in patients with TBM. Despite the serious consequences following the mycobacterial infection of the CNS, our understanding of the neuro- and immunopathogenesis of cerebral mycobacterial infection is limited.

Our laboratory, for the past decade, has been working on developing immunodiagnostic and molecular tools for the clinical evaluation and appropriate management of TBM patients admitted to the Central India Institute of Medical Sciences (CIIMS); it is a tertiary healthcare facility providing medical services to the Central Indian population (Kashyap, et al., 2005; Kashyap, et al., 2006; Deshpande, et al., 2008). The majority (70\%) of those admitted here are neurology and neurosurgery patients.

In our experience, a considerable number of TBM patients do not respond to the treatment with the standard drug regimen and present neurological sequelae. The present work was thus planned as a retrospective study to assess the predictors of mortality or poor prognosis in patients diagnosed with TBM. 


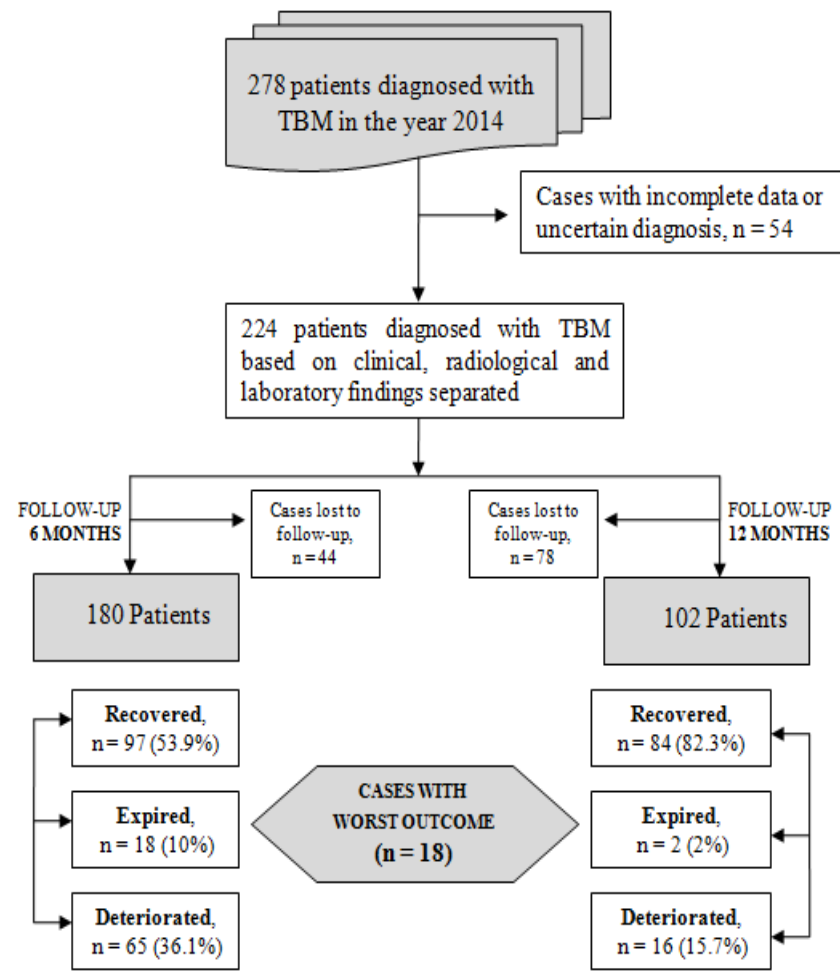

Figure 1. The schematic representation of the study design

\section{Materials and Methods}

The study was approved by the Institutional Ethics Committee of CIIMS, Nagpur City, India, and as per The Code of Ethics of the World Medical Association (the Declaration of Helsinki). Written informed consent forms were taken from each participant after providing a detailed oral explanation about the study project.

We retrospectively reviewed the medical files of all patients with the diagnosis of TBM admitted in 2014 to the CIIMS. From each medical case file, the patient's history, physical findings, chest radiographs, and the reports of laboratory investigations were assessed to obtain the necessary data about the diagnosis of TBM. For each patient, demographic information (age, gender), and clinical characteristics were also recorded.

The diagnosis of TBM was conducted according to internationally recognized clinical, radiological, and laboratory criteria (Torok, 2015). The clinical criteria included fever, headache, and neck stiffness.

The laboratory criteria included CSF pleocytosis of $>10$ cells per $\mathrm{mm} 3$ or proteins $>30 \mathrm{mg} / \mathrm{dL}$. The radiological criteria consisted of hydrocephalus, tuberculomas, cerebral infarcts, meningeal enhancements, or exudates. Additionally, the results of culture or staining of a clinical sample, Polymerase Chain Reaction (PCR) test, and Ag/ $\mathrm{Ab}$ test in the CSF, as well as tuberculin skin test were collected. A definite diagnosis of TBM was achieved when a positive culture or stain was present. A probable diagnosis was concluded when the clinical picture was compatible. A possible diagnosis was presented when there was a suggestive clinical picture and improvement with anti-TB treatment

In total, 278 cases were diagnosed with TBM in 2014 The cases with incomplete data or uncertain diagnosis were excluded from the study. Based on the abovementioned criteria, we separated 224 cases and followed them for 6 months and 12 months until 2015. These cases were clinically evaluated for new neurological complications, including the new formation or enlargement of cerebral lesions; the development of hydrocephalus; and the new onset of seizures or cranial nerve paralysis; the occurrence of intracranial hypertension; coma and death, or significant signs of improvement without new neurological deficits or symptoms.

Of the 224 TBM cases, 44 patients emigrated or were lost to follow-up within the first 6 months of diagnosis. Of the remaining 180 patients, $53.9 \%$ (97) of cases recovered completely, $36.1 \%$ (65) presented neurological deterioration, and $10 \%$ (18) died. 
Table 1. Primer sequences for RD region analysis

\begin{tabular}{|c|c|c|c|c|c|}
\hline PCR & Primers & Sequence & Annealing Temp & Amplicon Size & Reference \\
\hline RD4 & $\begin{array}{l}\mathrm{F} \\
\mathrm{R}\end{array}$ & $\begin{array}{l}\text { 5'-AATGGTTTGGTCATGACGCCTTC-3' } \\
\text { 5'-CCCGTAGCGTTACTGAGAAATTGC-3' }\end{array}$ & $58^{\circ} \mathrm{C}$ & $176 \mathrm{bp}$ & $\begin{array}{l}\text { Taylor } \\
\text { et al. (2007) }\end{array}$ \\
\hline RD1 & $\mathrm{F}$ & $\begin{array}{l}\text { 5'-CCCTTTCTCGTGTTTATAGTTTGA-3' } \\
\text { 5'-GCCATATCGTCCGGAGCTT-3' }\end{array}$ & $60^{\circ} \mathrm{C}$ & $110 \mathrm{bp}$ & $\begin{array}{l}\text { Halse, Escuyer, \& } \\
\text { Musser, (2011) }\end{array}$ \\
\hline
\end{tabular}

Table 2. The classification of study participants

\begin{tabular}{cc}
\hline Category * & No. of Cases \\
\hline Definite TBM & 24 \\
\hline Probable TBM & 123 \\
\hline Possible TBM & 77 \\
\hline Non-TBM & 34 \\
\hline
\end{tabular}

* Classification was performed on the basis of clinical, radiological, and CSF findings

NEUR SCIENCE

In a $12-$ month follow-up, these rates changed to $82.3 \%$ (84), 15.7\% (16), and 2\% (2) of recovered, deteriorated, and expired cases, respectively. The study workflow is represented in Figure 1. Along with the TBM group, a control group was included in the study, consisting of 34 non-TBM patients with viral (15) and fungal (4) meningitis, and patients with non-infectious illnesses of the CNS (15).

The potential for detecting mixed/new pathogenic infections in patients with poor prognosis led to the formulation of a novel strategy for differential diagnosis against the pathogenic mycobacteria. For this purpose, the Cerebrospinal Fluid (CSF) samples of TBM and non-TBM patients were processed for accurate identification of mycobacterial species; we used a duplex PCR assay targeting the Regions of Difference (RD) 1 and 4 in a single reaction.

CSF samples were collected under aseptic conditions by standard lumbar puncture. Five hundred microliter to $1 \mathrm{~mL}$ of sample was available for the study. The collected samples were stored at $-20^{\circ} \mathrm{C}$, prior to processing for target DNA for duplex PCR and culturing in the BACT alert system.

DNA was extracted from CSF samples by modifying the phenol-chloroform extraction method described by Deshpande et al. (2007); wherein $500 \mu \mathrm{L}$ of the sample was centrifuged at $12000 \mathrm{rpm}$ for $10 \mathrm{~min}$. The supernatant was discarded and the pellet suspended in $500 \mu \mathrm{L}$ of PBS, $15 \mu \mathrm{L}$
$10 \% \mathrm{SDS}$, and $3 \mu \mathrm{L}$ proteinase $\mathrm{K}(20 \mathrm{mg} / \mathrm{mL})$, mixed and incubated at $55^{\circ} \mathrm{C}$ for 1.5 hours. After incubation, $100 \mu \mathrm{L}$ of $5 \mathrm{M} \mathrm{NaCl}$ and $80 \mu \mathrm{L}$ of high-salt CTAB buffer (containing 4 $\mathrm{M} \mathrm{NaCl}, 1.8 \%$ CTAB (cetyl-trimethyl-ammonium bromide) was added and mixed; this process was followed by incubation at $65^{\circ} \mathrm{C}$ for $10 \mathrm{~min}$. An approximate equal volume (350 $\mu \mathrm{L})$ of phenol and chloroform-isoamyl alcohol (24:1) was added. Then, it was mixed thoroughly and centrifuged for 10 min in a microcentrifuge at 12000 RPM. The aqueous viscous supernatant was carefully decanted and transferred to a new tube. An equal volume of phenol: chloroform-isoamyl alcohol (1:1) was added followed by a $10 \mathrm{~min}$ spin at 12,000 RPM. The aqueous layer was separated. It was then mixed with $30 \mu \mathrm{L}$ of $3 \mathrm{M}$ sodium acetate and 0.6 volume of isopropanol to get a precipitate. The precipitated nucleic acids were washed with $70 \%$ ethanol, dried, and re-suspended in $30 \mu \mathrm{L}$ of Tris-EDTA (TE) buffer and stored at $-20^{\circ} \mathrm{C}$ before use. The DNA concentrations for all samples and strains used in this study were determined with the Quant-iTdsDNA HS assay kit using a Qubit fluorometer (Invitrogen).

For determining the species level of the mycobacterial pathogens, namely, Mycobacterium tuberculosis (M.tb), Mycobacterium Bovis (M. Bovis), and M. Bovis Bacilli Calmette Guérin (BCG), two genetic regions RD4 and RD1 were amplified using a duplex approach. Primers used in this study are presented in Table 1. 
Table 3. The clinical characteristics of the recruited participants

\begin{tabular}{|c|c|c|c|c|}
\hline \multirow{2}{*}{\multicolumn{2}{|c|}{ Characteristics }} & \multicolumn{2}{|c|}{ No. (\%)/ Mean士SD } & \multirow{2}{*}{$\mathbf{P}$} \\
\hline & & TBM Cases $(n=224)$ & Non-TBM Cases $(n=34)$ & \\
\hline & Male & $134(59.82)$ & $30(88.24)$ & 0.0045 \\
\hline \multirow[t]{2}{*}{ Gender } & Female & $90(40.18)$ & $4(11.76)$ & 0.0045 \\
\hline & Median age (y) & $37.19 \pm 17.67$ & $37.01 \pm 16.51$ & 0.9584 \\
\hline \multirow{8}{*}{ Clinical data } & Headache & 1655 (73.5) & $13(38.2)$ & 0.0004 \\
\hline & Fever & $134(59.8)$ & $10(29.4)$ & 0.0040 \\
\hline & Seizure & $46(20.5)$ & $3(8.8)$ & 0.1925 \\
\hline & Altered consciousness & $26(11.6)$ & $3(8.8)$ & 0.8671 \\
\hline & Altered sensorium & $99(44.2)$ & $6(17.6)$ & 0.0104 \\
\hline & Hallucinations & $6(2.7)$ & $2(5.9)$ & 0.7796 \\
\hline & Neck stiffness & $123(54.9)$ & $4(11.8)$ & $<0.0001$ \\
\hline & The duration of hospital stay & 30 & 15 & \\
\hline \multirow{2}{*}{ Radiological findings } & Hydrocephalus & $22(9.8)$ & $2(5.9)$ & 0.7299 \\
\hline & Meningeal enhancement & $70(31.3)$ & $6(17.6)$ & 0.1828 \\
\hline \multirow{3}{*}{ Laboratory results } & Proteins & 128.97 & 91.84 & 0.0322 \\
\hline & Glucose & 31.93 & 60.38 & $<0.0001$ \\
\hline & Cells & 209.72 & 83.75 & 0.0003 \\
\hline
\end{tabular}

RD4 is a region of difference in the bovine lineage. Employing RD4 flanking primers ensured that the PCR products were formed only if the deletion was present (Taylor, Worth, Palmer, Jahans, \& Hewinson, 2007). The genes of the $\mathrm{RD} 1$ region belong to the esat 6 gene cluster. ESAT-6 is a potent stimulator of the immune system, i.e. an antigen recognized during the early stages of infection. The RD1 region of M.tb is considered to be the primary attenuating deletion in the related vaccine strain M. bovis BCG (Halse, Escuyer, \& Musser, 2011) (Figure 2).

The duplex PCR reactions were conducted using 10X PCR buffer, $1.5 \mathrm{MgCl} 2,0.8 \mathrm{mM}$ dNTPs, $0.4 \mu \mathrm{M}$ of RD1F/R and $0.2 \mu \mathrm{M}$ of $\mathrm{RD} 4 \mathrm{~F} / \mathrm{R}$, and $1.25 \mathrm{U}$ of Taq DNA polymerase. The amplification procedure consisted of initial denaturation at $95^{\circ} \mathrm{C}$ for $7 \mathrm{~min}$ and 35 cycles each of denaturation at $95^{\circ} \mathrm{C}$ for $1 \mathrm{~min}$, annealing at $59^{\circ} \mathrm{C}$ for $1 \mathrm{~min}$, and extension at $72^{\circ} \mathrm{C}$ for $1 \mathrm{~min}$, followed by a final extension step at $72^{\circ} \mathrm{C}$ for $10 \mathrm{~min}$.

The sensitivity of the method was determined using serially diluted purified genomic DNA solutions-tenfold dilu- tion from $10 \mathrm{ng} / \mu \mathrm{L}$ to $1 \mathrm{FG} / \mu \mathrm{L}$; it was extracted from M.tb (ATCC 25177), M. Bovis (ATCC BAA-935), and M. Bovis BCG Pasteur (ATCC 35734). For the specificity study, the concentration of the DNA solution from each reference strain was adjusted to10 $\mathrm{ng} / \mu \mathrm{L}$, and used, accordingly.

The PCR amplicons were analyzed on a $2 \%$ agarose gel and stained with ethidium bromide. The amplified products were then visualized under UV light. Comparative analysis of electrophoresis of the PCR products generated by the two sets of primer pairs demonstrated the ability to distinguish between M.tuberculosis, M. bovis, and M. bovis BCG. The duplex PCR was considered as positive for M. bovis when the bands of 176-bp and 110-bp were detected; positive for M. Bovis BCG when the band of only 176-bp was present and positive for $\mathrm{M}$. TB when the band of only 110-bp was present.

The PCR products were purified and sequenced by Sanger's dideoxy chain termination method at the SciGenom Labs, Cochin, India. Sequences were verified by 


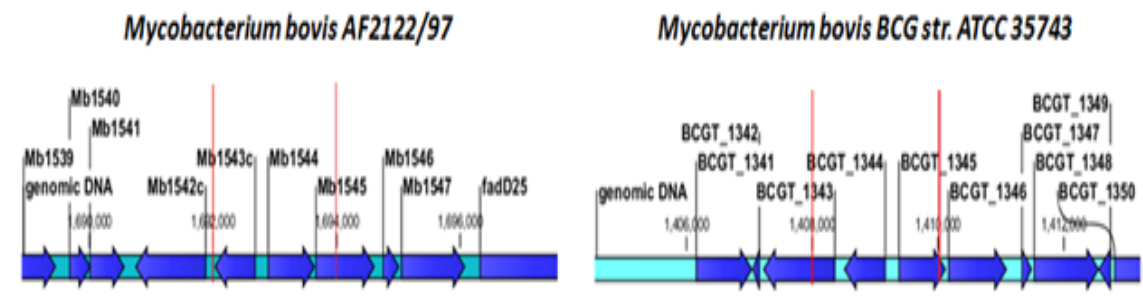

RD4 region of Mbovis and Mbovis BCG genomes giving 176-bp amplification product.

Mycobacterium bovis AF2122/97

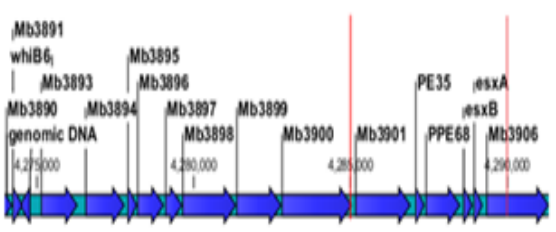

Mycobacterium tuberculosis CP009427

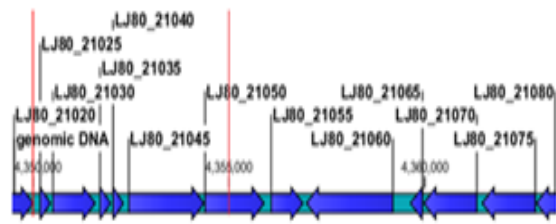

RD1 region of Mbovis and Mtuberculosis genomes giving 110-bp 176-bp amplification product.

NEUR SCIENCE

Figure 2. Molecular analysis for the identification and differentiation of Mycobacterium bovis (M. bovis), Mycobacterium bovisBCG (M. bovisBCG), and Mycobacterium tuberculosis (M.tb) using duplex PCR

* The position of the primers in the genomes is depicted.

BLAST search using the NCBI website. The positive culture isolates were subjected to antibiotic sensitivity using the 5 first-line drugs viz; Streptomycin, Isoniazid, Rifampicin, Ethambutol, and Pyrazinamide (SIREP) at Metropolis Healthcare Limited, Mumbai. In-vitro drug susceptibility testing was performed by incorporating required drug concentration and the subsequent inoculation of modified Middlebrook $7 \mathrm{H} 9$ Broth with standardized inoculum and incubated at $35^{\circ} \mathrm{C}$ in the automated BACTEC MGIT 960 system. Strains were declared resistant if the growth of $>20$ colonies was observed at drug concentrations as described by Rai, Bhattacharya, and Kamal (2007).

The recorded demographic and clinical characteristics were compared between TBM and non-TBM groups. The Chi-squared test for categorical variables was used to test the differences between the groups. Besides, $\mathrm{P} \leq$ 0.05 was considered as statistically significant. All tests were performed using MedCalc statistical software.

\section{Results}

Based on the clinical, radiological, and CSF findings, the recruited participants were categorized into definitive TBM $(n=24)$, probable TBM $(n=123)$, possible $\operatorname{TBM}(\mathrm{n}=77)$, and non-TBM cases $(\mathrm{n}=34)$ (Table 2$)$.

The overall male to female ratio of recruited cases was $1.7(164 / 94)$. For the TBM patients, the male to female ratio was $1.5(134 / 90)$, and 7.5 (30/4) for the non-TBM cases. The relevant difference was statistically significant $(\mathrm{P}<0.05)$. The median age of the TBM patients $(37.19$ years) and that of the non-TBM cases (37.01 years) was nearly similar (Table 3 ). A significantly higher proportion of the TBM cases experienced headache, fever, altered sensorium, and neck stiffness, compared to the non-TBM cases $(\mathrm{P}<0.01)$. On the other hand, a higher proportion of the non-TBM cases encountered hallucinations, compared to the TBM ones. A higher proportion of TBM cases had seizures and altered consciousness, compared to the non-TBM cases; however, such a difference was not statistically significant. The mean duration of illness was also significantly higher for TBM cases, compared to the non-TBM ones $(\mathrm{P}<0.0001)$. The frequency of cases that presented hydrocephalus and meningeal enhancement was also higher in the TBM cases, compared to the non-TBM cases. Laboratory findings indicated that the mean levels of protein and cell count in the TBM cases were significantly higher than those in the non-TBM cases $(\mathrm{P}<0.05)$. The glucose levels, however, were significantly lower in the non-TBM cases, compared to the TBM cases $(\mathrm{P}<0.0001)$.

Out of the total 224 TBM cases, 180 and 102 patients were followed up for 6 and 12 months, respectively. The radiological findings at the 6-month follow-up of the cases indicated that $22.8 \%$ had hydrocephalus, $3.9 \%$ had meningeal enhancements, and 1 case encountered stroke. 
Table 4. The follow-up clinical and radiological findings of the TBM cases

\begin{tabular}{|c|c|c|c|}
\hline & \multirow{2}{*}{ Characteristics } & \multicolumn{2}{|c|}{ No. (\%) } \\
\hline & & Follow-up* (6 M onths) & Follow-up" (12 Months) \\
\hline \multirow{3}{*}{ Clinical Findings } & Neurological Sequelae/Deterioration & $65(36.1)$ & $16(15.7)$ \\
\hline & Death & $18(10)$ & $2(2)$ \\
\hline & Improvement & $97(53.9)$ & $84(82.3)$ \\
\hline \multirow{3}{*}{ Radiological findings } & Hydrocephalus & $41(22.8)$ & $5(4.9)$ \\
\hline & Meningeal enhancement & $7(3.9)$ & $13(12.7)$ \\
\hline & Stroke & $1(0.6)$ & (0) \\
\hline \multirow{3}{*}{ Laboratory findings } & Proteins $>100 \mathrm{mg}$ & $48^{\natural}(26.7)$ & $18^{\text {" }}(17.6)$ \\
\hline & Glucose $<2 / 3$ CBS & $23^{\pi}(12.8)$ & $15^{\pi}(14.7)$ \\
\hline & Cells $>20$ cells $/ \mathrm{mm} 3$ & $34^{\uparrow}(18.9)$ & 13" (12.7) \\
\hline
\end{tabular}

NEUR SCIENCE

* $\mathrm{n}$ : 180 (44 cases lost to follow-up); ${ }^{n}$ : 102 (18 cases expired, lost to follow-up); " CSF was collected from patients with neurological deterioration.

In the second follow-up, $4.9 \%$ of the cases presented hydrocephalus, and $12.7 \%$ had meningeal enhancement. Protein, glucose, and cell count were found to be abnormal in $26.7 \%, 12.8 \%$, and $18.9 \%$ of the studied cases at the 6-month follow-up. At the 12-month follow-up step, these rates changed to $17.6 \%, 14.7 \%$, and $12.7 \%$ for protein, glucose, and total cell count, respectively. Table 4 indicates the follow-up clinical and radiological findings of the TBM cases.

The detection and differentiation of M.tb and M .bovis in CSF samples are represented in Figure 3. The PCR products in these samples were found to align with the
RD4 and RD1 regions of M. bovis (Figures 4A \& 4B). Of the 258 samples, $103(39.9 \%)$ were positive by the duplex PCR assay. M. bovis was detected in 7\% (18) of the investigated samples. Infection with M.tb was detected in $32.9 \%$ (85) of the samples. Furthermore, 14\% of the explored samples were positive for Bactec culture, M.tb or M. bovis; however, they could not be differentiated to the species level of the mycobacterial pathogens. The comparative efficiency of duplex PCR and Bactec culture for detecting mycobacteria are listed in Table 5 .

In-vitro drug susceptibility testing to first-line antitubercular drugs (SIREP) was outperformed and the sus-

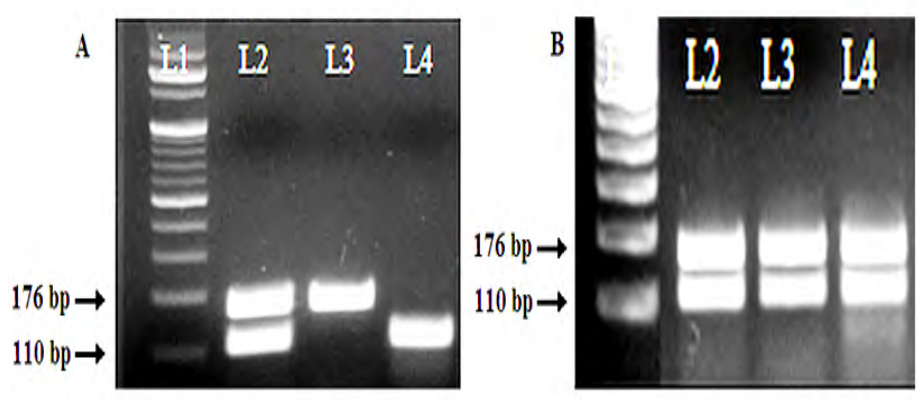

NEUROSCIENCE

Figure 3. Duplex PCR for detecting and differentiating M. bovis, M.tuberculosis, and M. bovis BCG.

A: The ethidium bromide-stained amplification products of L1: M. bovis, L2: M. bovisBCG and L3: M. tuberculosis when electrophoresced on $2 \%$ agarose gel. The $176 \mathrm{bp}$ and $110 \mathrm{bp}$ products obtained are indicated. B: L1: $100 \mathrm{bp}$ molecular ladder, L2: Positive control, L3 and L4: CSF samples with M. bovis infection. 


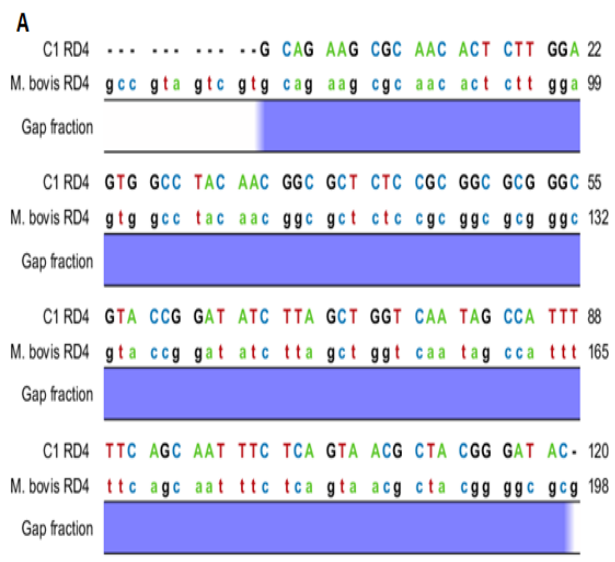

B

C1RD1 . G ACT ACG ACA AGC TCT TCC GGC 22

M. bovisRD1 ccg act acg aca agc tct tcc ggc 120

Consensus CCG ACT ACG ACA AGC TCT TCC GGC

Conservation

C1RD1 CGC ACG AAG GTA TGG AAG CTC CGG 46

M. bovisRD1 cgc acg aag gta $\operatorname{tgg}$ aag ctc cgg 144

Consensus CGC ACG AAG GTA TGG AAG CTC CGG

Conservation

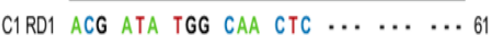

M. bovisRD1 acg ata $\operatorname{tgg} \mathrm{cag} \mathrm{cgc}$ agc cgt tct 168

Consensus ACG ATA TGG CAN CNC AGC CGT TCT

Conservation

NEUR SCIENCE

Figure 4. The follow-up clinical and radiological findings of the TBM cases

A: The alignment of M. bovis RD4 region and the PCR product of duplex PCR targeting the RD4 region in clinical sample from TBM patient (C1);

B. The alignment of M. bovis RD1 region and the PCR product of duplex PCR targeting the RD1 region in the same sample (C1)

*Sequence alignment was conducted using CLC Sequence Viewer, Version 6.6.1.

ceptibility pattern is detailed in Table 6 . One of the culture isolates was found to be resistant to pyrazinamide at a critical concentration of $100 \mu \mathrm{g} / \mathrm{mL}$. No other isolate reflected resistance to the first-line antitubercular drugs.

\section{Discussion}

The diagnosis of TBM has remained a challenge due to the low-efficiency and prolonged-time required by culture methods and conventional biochemical techniques. The paucibacillary nature of CSF has also been an additional impediment in the accurate diagnosis of the disease (Rock, Olin, Baker, Molitor, \& Peterson; Marx \& Chan, 2011).

We initiated this study to understand the variety of the presentations of TBM, including fatal and irrevocable effects occurring in a significant number of cases. We selected those patients diagnosed with TBM and followed them for one year to assess their neurological condition. With an underlying objective to clinically evaluate TBM patients with poor prognosis, we developed an assay based on the molecular detection of RD regions to accurately identify the causative organism in the CSF samples of these patients.

To our surprise, we found that $7 \%(18 / 258)$ of the cases diagnosed with TBM were infected with M. bovis, the classical causative agent of bovine TB; also reportedly responsible for human TB, which makes this bacterium an important zoonotic pathogen (Allix-Béguec, et al., 2010). In developed countries, the introduction of pasteurization and eradication programs for infected herds have considerably reduced the prevalence of human disease due to the bovine TB bacillus; however, have not completely eradicated it (Evans, et al., 2007). In developing countries, however, $\mathrm{M}$. bovis has been reported to account for approximately $10 \%$ to $15 \%$ of new human TB cases (Ashford, Whitney, \& Raghunathan, \& Cosivi, 2001).

The classical biochemical tests for identifying M. bovis and molecular methods based on targets, such as IS6110, 16S rDNA, 23S rDNA, or ITS cannot distinguish between $\mathrm{M}$. bovis and the other M.tbcomplex (MTBC) members. Other genetic markers and the single commercial test (GenoType Mycobacterium, Hain, Nehren, Germany) allowing the distinction between MTBC members, are not widely used due to the associated high costs (Sansila, et al., 1998). Diagnostically, the low sensitivity of CSF TB PCR is also problematic. Potential explanations for the lack of sensitivity in CSF specimens include low bacillary load in CSF, small sample volumes, and PCR inhibitors in the samples (Christie, et al., 2008). Moreover, from the clinicians' viewpoint, TB caused by M.tb. in humans, is clinically and radiologically identical to TB, caused by M. bovis (Grange, 2001). The distinction of M. bovis from M.tb however, has significant relevance to patient management. This is because M. bovis is intrinsically resistant to pyrazinamide; the absence of specific identification may have adverse consequences for infected cases (Niemann, Richter, \& Rüsch- Gerdes, 
Table 5. The comparative efficiency of duplex PCR and Bactec culture for the detection of mycobacteria

\begin{tabular}{cccccc}
\hline & \multicolumn{3}{c}{ No.(\%) } \\
\cline { 2 - 5 } Duplex PCR Result & \multirow{2}{c}{ Species Identified } & \multicolumn{2}{c}{ Culture Result } \\
\cline { 3 - 6 } & PCR Result & M.tuberculosis & M. bovis & Positive & Negative \\
\cline { 3 - 6 } & & $85(32.9)$ & $18(7.0)$ & $28(27.2)$ & $75(72.8)$ \\
Positive & $103(39.9)$ & $173(67.1)$ & $240(93.0)$ & $8(5.2)$ & $147(94.8)$ \\
Negative & $155(60.1)$ & $258(100)$ & $258(100)$ & $36(14)$ & $222(86)$ \\
\hline Total & $258(100)$ & & & & NEUR:SCIENCE
\end{tabular}

Table 6. The drug susceptibility testing of positive culture isolate

\begin{tabular}{ccc}
\hline Antitubercular Drug & Critical Concentration $(\mu \mathrm{g} / \mathrm{mL})$ & Interpretation \\
\hline Streptomycin & 1 & Susceptible \\
Isoniazid & 0.1 & Susceptible \\
Rifampicin & 1 & Susceptible \\
Ethambutol & 5 & Susceptible \\
Pyrazinamide & 100 & Resistant \\
\hline
\end{tabular}

2000). Identifying this pathogen would thus help clinicians to adopt desirable patient management concerning the treatment regimen. Reports by Allix-Béguec, et al. (2010) and Hannan, et al. (2001) have highlighted the significance of the routine use of molecular tests for the differentiation of M. bovis from M.tb or the systematic checking of resistance to pyrazinamide.

Various studies reported the presence of M. bovis infection in humans (Kidane, et al, 2002; Wei, Huang, Chu, Lee, 1999; LoBue, Betacourt, \& Peter, 2003; Cosivi, et al., 1998). In the Indian context, a study by Jain (2011) demonstrated M. bovis infection in $9.52 \%$ of the cases and the co-infection of M.tb and M. bovis in $4.76 \%$ of the cases through a two-step PCR, targeting the hup B gene. Prasad et al. (2005) reported that 34.7\% PCR positivity, of which M.tb was detected in $15.7 \%$ of the cases, M. bovis was observed in $10.3 \%$, and mixed infection in $8.7 \%$ of the samples. Shah et al. (2006) described applying a nested PCR (N-PCR) assay in detecting M.tb and M. bovis in human CSF. They reported the presence of M. bovis in $17 \%$ of the cases and mixed infection in $22 \%$ of them.

In the present study, of the total 258 cases, we detected M. bovis in 18 (7\%) cases; of which, two suffered a fatal outcome. One case; a 24-year-old pregnant woman
(ANC 26 weeks), was admitted with the typical symptoms of TBM, including altered sensorium, intermittent fever, decreased appetite, vomiting, and severe headache. Her CT scan presented meningeal enhancement, chest X-ray indicated Koch's infiltrations and subsequently initiated anti-TB treatment. The patient delivered a healthy premature baby during the hospital course and was discharged, subsequently. However, after 6 months, she re-developed symptoms, including abnormal behavior, marked neck-stiffness, and drowsiness. The patient was readmitted and surgically treated. The patient had however expired before the 12-month follow-up. Regardless and more importantly, the initial absence of $\mathrm{M}$. bovis identification of this patient's isolate compromised the efficiency of her treatments and plausibly influenced the final fatal outcome.

The second case, a 68-year-old male, had a history of right-sided pyothorax (tubercular) and infarction in corona radiata. He was discharged against medical advice from a local hospital, and consequently, admitted to CIIMS. The patient, upon admission, initiated anti-TB treatment and was discharged on improvement. The patient presented neurological sequelae upon follow-up and was readmitted; however, he suffered septic shock and died of multi-organ failure. In the remaining M. bovis in- 
fected cases, also the standard treatment regimen proved to be ineffective. It has led to neurological deterioration with symptoms, such as slurred speech, decreased hearing, and difficulty in walking. The assessment of the demographic characteristics of these individuals indicated that all the patients belonged to rural settings, where agriculture was the main source of livelihood. All the subjects had occupational exposure to farm animals. Close physical contact with animals and the consumption of unpasteurized milk could be the potential source of $\mathrm{M}$. bovis transmission in these individuals. Michel, Muller, and Van Helden (2010) suggested that pastoralist and rural communities are at greatest risk for zoonotic TB; however, the lack of data for these populations prevents the confirmation of this assumption.

While several articles have described the epidemiology, clinical features, and transmission of $\mathrm{M}$. bovis disease in humans, little has been documented about its treatment (LoBue \& Moser, 2005). Beyond its use for specific M. bovis identification, this natural resistance is particularly important to consider. Pyrazinamide is usually given in the classical first-line TB treatment; it is an effective sterilizing drug that helps to shorten TB therapy due to its synergistic effect with rifampicin. Thus, in the case of $M$. bovis infection, pyrazinamide would be ineffective if implemented in a patient's anti-TB regimen. The duration of treatment thus tended to be longer for patients with $\mathrm{M}$. bovis infection; they were considered ineligible for a 6-month treatment regimen due to PZA resistance (LoBue \& Moser, 2005). LoBue et al. also revealed that the death rate was highest for $\mathrm{M}$. bovis patients (LoBue \& Moser, 2005). In the present study, given the high rates of $\mathrm{M}$. bovis in TBM patients, the surveillance of this zoonotic pathogen was obviated.

\section{Conclusion}

Our study findings demonstrated the prevalence of $\mathrm{M}$. bovis-induced TB in patients with poor therapeutic outcomes. Besides, our report constituted an example of the persistent significance of $\mathrm{M}$. bovis, as a zoonotic pathogen. Human TB due to M. bovis is underestimated. This is because of the frequent use of diagnostic techniques that do not specifically distinguish M. bovis from other members of the M. tuberculosis complex, and because susceptibility to pyrazinamide is not systematically tested. Finally, the data demonstrated that molecular-guided cooperation between human and veterinary health services could improve the detection of zoonoses in the future.

\section{Ethical Considerations}

\section{Compliance with ethical guidelines}

The study was approved by Institutional Ethics Committee of Central India Institute of Medical Sciences (CIIMS), Nagpur and is in accordance with the Code of Ethics of the World Medical Association (Declaration of Helsinki). Written consents were taken from each participant or their kin after detailed oral explanation about the study.

\section{Funding}

This work was supported by the Indian Council of Medical Research (ICMR), New Delhi, India (Grant No: Zon.15/11/2014-ECD-II).

\section{Authors' contributions}

All authors were equally contributed in preparing this article.

\section{Conflict of interest}

The authors declared no conflicts of interest.

\section{Acknowledgments}

The authors acknowledge Dr. Dewanand R. Kalorey, Dr. Nitin V. Kurkure, and Dr. Sandeep P. Chaudhari for their support. The authors would also like to thank Ms. Sonali Manke and Ms. Ankita Tiwari for their technical assistance.

\section{References}

Ahmadinejad, Z., Ziaee, V., Aghsaeifar, M., \& Reiskarami, S. R. (2003). The prognostic factors of tuberculous meningitis. International Journal of Infectious Diseases, 3(1), 1-7. [DOI:10.5580/3a0]

Allix-Béguec, C., Fauville-Dufaux, M., Stoffels, K., Ommeslag, D., Walravens, K., Saegerman, C., \& Supply, P. (2010). Importance of identifying Mycobacterium bovis as a causative agent of human tuberculosis. European Respiratory Journal, 35(3), 692-4 [DOI:10.1183/09031936.00137309] [PMID]

Ashford, D. A., Whitney, E., Raghunathan, P., \& Cosivi, O. (2001). Epidemiology of selected mycobacteria that infect humans and other animals. Revue scientifique et technique (International Office of Epizootics), 20(1), 325-37. [DOI:10.20506/rst.20.1.1266] [PMID]

Christie, L. J., Loeffler, A. M., Honarmand, S., Flood, J. M., Baxter, R., \& Jacobson, S., et al. (2008). Diagnostic challenges of central nervous system tuberculosis. Emerging Infectious Diseases, 14(9), 1473. [DOI:10.3201/eid1409.070264] [PMID] [PMCID] 
Cosivi, O., Grange, J. M., Daborn, C. J., Raviglione, M. C., Fujikura, T., \& Cousins, D., et al. (1998). Zoonotic tuberculosis due to Mycobacterium bovis in developing countries. Emerging Infectious Diseases, 4(1), 59-70. [DOI:10.3201/eid0401.980108] [PMID] [PMCID]

Deshpande, P. S., Kashyap, R. S., Ramteke, S. S., Nagdev, K. J., Purohit, H. J., Taori, G. M., \& Daginawala, H. F. (2007). Evaluation of the IS6110 PCR assay for the rapid diagnosis of tuberculous meningitis. Cerebrospinal Fluid Research, 4(1), 10. https:// link.springer.com/article/10.1186/1743-8454-4-10

Evans, J. T., Smith, E. G., Banerjee, A., Smith, R. M., Dale, J., \& Innes, J. A., et al. (2007). Cluster of human tuberculosis caused by Mycobacterium bovis: Evidence for person-to-person transmission in the UK. The Lancet, 369(9569), 1270-6. [DOI:10.1016/S01406736(07)60598-4]

World Health Organization. (2020). Global tuberculosis report 2015 - World Health Organization; Geneva: World Health Organization. https://www.who.int/emergencies/diseases/novel-

Grange, J. M. (2001). Mycobacterium bovis infection in human beings. Tuberculosis, 81(1-2), 71-7. [DOI:10.1054/tube.2000.0263] [PMID]

Halse, T. A., Escuyer, V. E., \& Musser, K. A. (2011). Evaluation of a single-tube multiplex real-time PCR for differentiation of members of the Mycobacterium tuberculosis complex in clinical specimens. Journal of Clinical Microbiology, 49(7), 2562-7. [DOI:10.1128/ JCM.00467-11] [PMID] [PMCID]

Hannan, M. M., Desmond, E. P., Morlock, G. P., Mazurek, G. H., \& Crawford, J. T. (2001). Pyrazinamide-monoresistant Mycobacterium tuberculosis in the United States. Journal of Clinical Microbiology, 39(2), 647-50. [DOI:10.1128/JCM.39.2.647-650.2001] [PMID] [PMCID]

Jain, A. (2011). Extra pulmonary tuberculosis: A diagnostic dilemma. Indian Journal of Clinical Biochemistry, 26(3), 269-73. [DOI:10.1007/s12291-010-0104-0] [PMID] [PMCID]

Kashyap, R. S., Dobos, K. M., Belisle, J. T., Purohit, H. J., Chandak, N. H., \& Taori, G. M., et al. (2005). Demonstration of components of antigen 85 complex in cerebrospinal fluid of tuberculous meningitis patients. Clinical and Diagnostic Laboratory Immunology, 12(6), 752-8. [DOI:10.1128/CDLI.12.6.752-758.2005] [PMID] [PMCID]

Kashyap, R. S., Kainthla, R. P., Mudaliar, A. V., Purohit, H. J., Taori, G. M., \& Daginawala, H. F. (2006). Cerebrospinal fluid adenosine deaminase activity: A complimentary tool in the early diagnosis of tuberculous meningitis. Cerebrospinal fluid research, 3(1), 5. https://link.springer.com/article/10.1186/1743-8454-3-5

Kaur, H., Sharma, K., Modi, M., Sharma, A., Rana, S., \& Khandelwal, N., et al. (2015). Prospective analysis of 55 cases of Tuberculosis Meningitis (TBM) in North India. Journal of Clinical and Diagnostic Research, 9(1), DC15. [DOI:10.7860/JCDR/2015/11456.5454] [PMID] [PMCID]

Kidane, D., Olobo, J. O., Habte, A., Negesse, Y., \& Aseffa, A., Abate, G., et al. (2002). Identification of the causative organism of tuberculous lymphadenitis in Ethiopia by PCR. Journal of Clinical Microbiology, 40(11), 4230-4. [DOI:10.1128/JCM.40.11.42304234.2002] [PMID] [PMCID]

LoBue, P. A., \& Moser, K. S. (2005). Treatment of mycobacterium bovis infected tuberculosis patients: San Diego County, Califor- nia, United States, 1994-2003. The International Journal of Tuberculosis and Lung Disease, 9(3), 333-8. https://www.ingentaconnect. com/content/iuatld/iitld/2005/00000009/00000003/art00019

LoBue P, A., Betacourt, W., Peter, C., \& Moser K, S. (2003). Epidemiology of Mycobacterium bovis disease in San Diego County, 1994-2000. The International Journal of Tuberculosis and Lung Disease, 7(2), 180-5. https://www.ingentaconnect.com/ content/iuatld/ijtld/2003/00000007/00000002/art00013

Marx, G. E., \& Chan, E. D. (2011). Tuberculous meningitis: diagnosis and treatment overview. Tuberculosis Research and Treat ment, 2011. [DOI:10.1155/2011/798764] [PMID] [PMCID]

Michel, A. L., Müller, B., \& Van Helden, P. D. (2010). Mycobacterium bovis at the animal-human interface: A problem, or not? Veterinary Microbiology, 140(3-4), 371-381. [DOI:10.1016/j. vetmic.2009.08.029] [PMID]

Murthy, J. M. K. (2010). Tuberculous meningitis: The challenges. Neurology India, 58(5), 716. [DOI:10.4103/0028-3886.72178] [PMID]

Niemann, S., Richter, E., \& Rüsch-Gerdes, S. (2000). Differentiation among Members of the Mycobacterium tuberculosis Complex by Molecular and Biochemical Features: Evidence for Two Pyrazinamide-Susceptible Subtypes of M. bovis. Journal of Clinical Microbiology, 38(1), 152-7. https://jcm.asm. org/content/38/1/152.short

Prasad, H. K., Singhal, A., Mishra, A., Shah, N. P., Katoch, V. M., \& Thakral, S. S., et al. (2005). Bovine tuberculosis in India: potential basis for zoonosis. Tuberculosis, 85(5-6), 421-8. [DOI:10.1016/j.tube.2005.08.005] [PMID]

Purohit, M., and Mustafa, T. (2015). Laboratory Diagnosis of Extra-pulmonary Tuberculosis (EPTB) in Resource-constrained Setting: State of the Art, Challenges and the Need. Journal of Clinical and Diagnostic Research, 9(4), EE01-EE06. [DOI:10.7860/JCDR/2015/12422.5792] [PMID] [PMCID]

Rai, S. P., Bhattacharyya, D., \& Kashyap, M. (2007). Pattern of initial drug resistance and its impact on short course chemotherapy of pulmonary tuberculosis. Lung India, 24(2), 51-3. [DOI:10.4103/0970-2113.44210]

Rock, R. B., Olin, M., Baker, C. A., Molitor, T. W., \& Peterson, P. K. (2008). Central nervous system tuberculosis: pathogenesis and clinical aspects. Clinical Microbiology Reviews, 21(2), 243-61. [DOI:10.1128/CMR.00042-07] [PMID] [PMCID]

Sansila, A., Hongmanee, P., Chuchottaworn, C., Rienthong, S., Rienthong, D., \& Palittapongarnpim, P. (1998). Differentiation between Mycobacterium tuberculosis and Mycobacterium avium by Amplification of the 16S-23S Ribosomal DNA Spacer. Journal of Clinical Microbiology, 36(9), 2399-403. [DOI:10.1128/JCM.36.9.2399-2403.1998] [PMID] [PMCID]

Shah, N. P., Singhal, A., Jain, A., Kumar, P., Uppal, S. S., \& Srivatsava, M. V. P., et al. (2006). Occurrence of overlooked zoonotic tuberculosis: detection of Mycobacterium bovis in human cerebrospinal fluid. Journal of Clinical Microbiology, 44(4), 1352-8. [DOI:10.1128/JCM.44.4.1352-1358.2006] [PMID] [PMCID]

Taylor, G. M, Murphy, E., Hopkins, R., Rutland, P., Chistov, Y. (2007). First report of Mycobacterium bovis DNA in human remains from the Iron Age. Microbiology (Reading), 153(Pt 4), 1243-9. [DOI:10.1099/mic.0.2006/002154-0] [PMID] 
Taylor, G. M., Worth, D. R., Palmer, S., Jahans, K., \& Hewinson, R. G. (2007). Rapid detection of Mycobacterium bovis DNA in cattle lymph nodes with visible lesions using PCR. BMC Veterinary Research, 3(1), 1-11. [DOI:10.1186/1746-6148-3-12] [PMID] [PMCID]

Török, M. E. (2015). Tuberculous meningitis: Advances in diagnosis and treatment. British Medical Bulletin, 113(1), 117-31. [DOI:10.1093/bmb/ldv003] [PMID]

Wei, C. Y., Hwang, J. J., Chu, C. H., \& Lee, C. P. (1999). Detection and identification of Mycobacterium tuberculosis by nested PCR assays in cerebrospinal fluid samples from patients with suspected tuberculous meningitis. The Kaohsiung Journal of Medical Sciences, 15(8), 475-83. [DOI:10.6452/ KJMS.199908.0475] 


\title{
ResearchPaper:InvestigatingEffectiveFactorsonEstimated Hemorrhage Intraoperative in Brain Meningioma Surgery
}

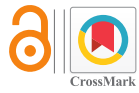

\author{
Alireza Tabibkhooei $^{1}$ (D), Maziar Azar ${ }^{2}$, Ahmad Alagha $^{{ }^{*}}$ (D), Javad Jahandideh ${ }^{1}$, Feyzollah Ebrahimnia ${ }^{1}$
}

1. Department of Neurosurgery, School of Medicine, Iran University of Medical Sciences, Tehran, Iran.

2. Skull Base Research Center, Iran University of Medical Sciences, Tehran, Iran.

\begin{tabular}{|l|l|}
\begin{tabular}{|l} 
Use yur devic to scan \\
and read the article online
\end{tabular} & $\begin{array}{l}\text { Cftat On: Tabibkhooei, A., Azar, M., Alagha, A., Jahandideh, J., \& Ebrahimnia, F. (2020). Investigating Effective Factors on } \\
\text { Estimated Hemorrhage Intraoperative in Brain Meningioma Surgery. Basic and Clinical Neuroscience, 11(5), 631-638. http:// } \\
\text { dx.doi.org/10.32598/bcn.9.10.370 }\end{array}$ \\
dof http://dx.doi.org/10.32598/bcn.9.10.370
\end{tabular}

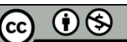

Article info:

Received: 09 Jun 2019

First Revision: 25 Jun 2019

Accepted: 26 Nov 2019

Available Online: 01 Sep 2020

Keywords:

Hemorrhage, Meningioma, Magnetic Resonance Imaging (MRI), Diagnosis

\section{A B S T RA C T}

Introduction: The primary and definitive diagnosis of meningioma is based on histological assessment; however, employing imaging methods, like Magnetic Resonance Imaging (MRI) is very helpful to describe lesion's characteristics. Accordingly, we decided to study the effect of imaging factors, like MRI data on the volume of hemorrhage (estimated blood loss) during meningioma surgery.

Methods: This was a cross-sectional, retrospective, and analytical study. The eligible patients were those with meningioma who were candidates for surgery. A total of 40 patients with meningioma were selected and assessed. The preoperative imaging findings were recorded, then estimated blood loss during the surgery was determined

Results: A reverse association was revealed between the degree of proximity to the nearest sinus and the rate of bleeding. Furthermore, the size of the mass was positively associated with the rate of bleeding; however, there was no significant correlation between the volume of bleeding and other parameters, including the degree of edema, the volume of mass, the site of the tumor in the brain, and the histological subtype of the tumor. The mean time of operation was strongly correlated with blood loss. The rate of bleeding was more expected in hypertensive versus normotensive patients.

Conclusion: Bleeding in various volumes could be a frequent finding in intracrania meningioma surgery. Overall, tumor size, the duration of surgery, a history of hypertension, and distance to the nearest sinuses were the main determinants for the severity of hemorrhage in patients undergoing meningioma surgery.

\section{* Corresponding Author:}




\section{Highlights}

- The size of the mass was positively associated with estimated blood loss volume in meningioma surgery.

- The mean time of operation was strongly correlated with the rate of bleeding in meningioma surgery.

- The closer a tumor to the sinuses, the more loss of blood volume will occur during operation in meningioma surgery.

- The rate of bleeding was expected to be higher in hypertensive patients under meningioma surgery.

\section{Plain Language Summary}

Meningioma is the most common tumor in the central nervous system. The primary and definitive diagnosis of meningitis is based on histological evaluation. Using imaging techniques, like Magnetic Resonance Imaging (MRI) can be helpful in preoperative diagnostic evaluations in patients undergoing the surgical management of meningioma. These modalities can provide good diagnostic performance to predict the operational risk as well as the likelihood of procedural complications, like brain hemorrhage. In this study, eligible patients were those with meningioma, who completely eliminated the lesion. The employed preoperative diagnostic method was MRI. The studied patients' demographic characteristics, including age, gender, preoperative laboratory examinations, history of cardiovascular disease, diabetes, and antiplatelet use, operation time, and total intraoperative blood loss were recorded. The tumor volume was measured in coronal, axial, and sagittal cuts in MRIs; we also evaluated parameters, such as maximum connection to the dura, tumor size, and distance to the nearest sinuses. Brain edema was determined as mild, moderate, and severe, according to its volume concerning tumor volume (less, equal, \& more in size, respectively). The results suggested no association between the rate of hemorrhage, and gender, age, history of diabetes, history of coronary disease, and history of anti-thrombocyte tumor size; however, the duration of surgery, history of hypertension, and distance to the nearest sinuses were the main determinants for the severity of hemorrhage in meningioma surgery.

\section{Introduction}

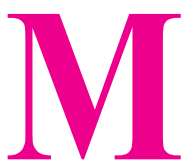

eningioma is the most prevalent tumor in the central nervous system; it accounts for between $16 \%$ and $20 \%$ of the total intracranial tumors (Toh et al., 2008). The Magnetic Resonance Imaging (MRI) technique is a selective modality for assessing meningioma. MIR not only presents the tumor's position with high contrast and can evaluate intra- and extra-axial lesions (Whittle, Smith, Navoo \& Collie, 2004; O’Leary, Adams, Parrish \& Mukonoweshuro, 2007; Nagar et al., 2008; Buetow, Buetow \& Smirniotopoulos;1991). In addition to MRI, Computed Tomography (CT) scan also plays an essential role in detecting calcification, as well as lesions associated with calvarium changes (Tokgoz et al., 2005). However, typical meningioma has well-known visual characteristics; there are also various atypical and unusual types that in addition to creating a diagnostic challenge for meningioma, have questioned the diagnostic accuracy of imaging techniques (Paek et al., 2005; Hakyemez et al., 2006). A radiologist must achieve a complete understanding of the atypical features of the disease in the images pro- vided to present the correct diagnosis (Elder, Atkinson, Zee \& Chen, 2007; Komotar, Keswani, Wityk, 2003).

In MRI, meningioma appears in the form of signal strength characteristics, including isointensity to mild hyperintensity, relative to gray matter, on the T2 sequence (Heye, Maleux, Van Loon \& Wilms, 2006). After applying contrast, meningioma appeared in the form of homogenous enhancement (Yue et al., 2008). However, the lesions occasionally exhibited necrosis or calcification, which in this case lacked enhancement. Calcification is visualized better in the CT scan view (Chen et al., 2004). On MRI view, especially on T2 sequences, calcification can be detected in the form of low-intensity signals (Bitzer et al., 1998). Contrast is also well-suited for detecting plaque-shaped meningioma, i.e. appeared as asymmetrical thickness on dura (Nakano, Nakano, Miura, Itoh \& Suzuki, 2002).

The primary and definitive diagnosis of meningioma is based on histological assessment. However, implementing imaging methods, like MRI has a special place in this field. Such approaches not only detect the nature of the tumor but also help detect local invasion, especially on 
T2-weighted images. Therefore, concerning non-invasive nature, imaging techniques could be beneficial in preoperative diagnostic evaluations in patients undergoing surgical management of meningioma. These modalities could provide desirable diagnostic performance to predict the operation risk, as well as the odds of procedural complications, like brain hemorrhage. Accordingly, we investigated the effect of imaging techniques, like MRI on the rate of hemorrhage during meningioma surgery.

\section{Materials and Methods}

This was an observational study, i.e. performed as a cross-sectional retrospective analytical study. Besides, the eligible patients were those with meningioma and totally removed lesion. The study exclusion criteria were contraindications for imaging with different methods, like MRI, and significant bony involvement by tumor. A total of 40 patients with meningioma admitted to Rasoul-e-Akram Hospital in Tehran City, Iran from 2015 to 2016 were selected and assessed in this research.

The demographic characteristics of the patients, including age, gender, preoperative laboratory examinations, a history of cardiovascular disease, diabetes, and anti-platelets use were collected from the patients' medical records. The operation time and total intraoperative blood loss were recorded from the patients' anesthesia file. The most frequent manifestation in the outcome analysis was a headache, followed by blurred vision, and limb weakness.

The preoperative data of the brain were received from MRI data. MRI examinations were obtained on a $1.5 \mathrm{~T}$ scanner (Philips Medical Systems, Ingenia). Imaging protocols in the T1 sequence were performed with contrast (slice thickness: $2 \mathrm{~mm}$ ). The location of the tumors was classified as parasagittal, parallax, convexity, and skull base (anterior, posterior, \& middle).

The tumor volume was measured and calculated using the ellipsoid formula,where A. The largest diameter of the tumor in the axial view; $b$. The tumor diameter in the same cut of the axial view perpendicular to $\mathrm{A}$; and $\mathrm{C}$. The tumor height using the number of cut counts that the tumor is observed in. Additionally, parameters, such as maximum connection to the dura, tumor size, and distance to nearest sinuses were measured.

The tumor size represents its diameter. The axial, coronal, and sagittal views were examined; the largest diameter of the tumor was specified in each.
Brain edema was determined as mild, moderate, and severe according to its volume respecting tumor volume (less, equal, and more in size, respectively). The preoperative imaging findings were recorded, then volume blood loss was evaluated during surgery. The frequency of hemorrhage cases was finally determined based on the imaging data.

The collected results were presented as Mean \pm Standard Deviation (SD) for quantitative variables. The study findings were summarized by absolute frequencies and percentages for categorical variables. The normality of the data was analyzed using the Kolmogorov-Smirnoff test. Categorical variables were compared using the Chisquared test or Fisher's Exact test when $>20 \%$ of the cells with an expected count of $<5$ were observed. The quantitative variables were also compared with the t-test or Mann-Whitney U test. The correlation between the quantitative parameters was examined by Pearson's correlation test. For the statistical analysis, SPSS was used. $\mathrm{P} \leq 0.05$ was considered as statistically significant.

\section{Results}

Totally, 40 patients with meningioma who underwent total surgical removal were assessed. Besides, the mean \pm SD age of patients was $54.90 \pm 12.66$ years, with a range of 28 to 77 years. In total, $72.5 \%$ of the study participants were female. Regarding cardiovascular risk factors, $30.0 \%$ were hypertensive, $12.5 \%$ were diabetic, $7.5 \%$ reported a history of coronary disease, and 7.5\% had a history of anti-platelets use. The most prevalent manifestation was headache $(67.5 \%)$, followed by blurred vision $(30.0 \%)$, and limb weakness $(25.0 \%)$. In imaging assessment, mild, moderate, and severe brain edema were revealed in $45.0 \%, 37.5 \%$, and $17.5 \%$ of the cases, respectively. Regarding the site of tumor, $25.0 \%$ was located in convexity, $45.0 \%$ in skull base $(72.2 \%$ in anterior \& $27.7 \%$ in posterior sites), and $30.0 \%$ in parasagittal regions. The Mean \pm SD size of meningioma was $41.72 \pm 19.42 \mathrm{~mm}$ with the Mean $\pm \mathrm{SD}$ volume of $49.36 \pm 61.90 \mathrm{~mm}^{3}$ (Table 1). Concerning the subtypes of meningioma, meningothelial type was detected in $35.0 \%$, transitional in $30.0 \%$, atypical in $10.0 \%$, cystic meningioma in 5.0\%, non-specified grade 1 in $5.0 \%$, angiomatosis in 5.0\%, psammomatous in $2.5 \%$, and microcystic in $5.0 \%$. The Mean \pm SD volume of hemorrhage was $925.64 \pm 756.19 \mathrm{~mL}$, and the $\mathrm{Mean} \pm \mathrm{SD}$ operation time equaled $7.15 \pm 2.03 \mathrm{~h}$. With regard to tumor distance to the nearest sinus, the proximity was found as $50.0 \%$, $15 \%$, and $35 \%$ for superior sagittal, transverse, and cavernous sinuses, respectively. 
Table 1. Meningioma characteristics observed in MRI findings

\begin{tabular}{|c|c|c|}
\hline & Variabels & No. (\%) \\
\hline \multirow{3}{*}{ Edema } & Mild & $18(45.0)$ \\
\hline & Moderate & $15(37.5)$ \\
\hline & Severe & $7(17.5)$ \\
\hline \multirow{8}{*}{ The location of the } & Convexity & $10(25.0)$ \\
\hline & Skull base & $18(45.0)$ \\
\hline & Ant Skull base & $13(72.2)$ \\
\hline & Pos Skull base & $5(27.7)$ \\
\hline & Parasagittal & $12(30.0)$ \\
\hline & Size of the tumor (Mean \pm SD) & $41.72 \pm 19.42$ \\
\hline & The volume of the tumor (Mean \pm SD) & $49.36 \pm 61.90$ \\
\hline & Maximum connection to the dura (Mean \pm SD) & $39.44 \pm 21.14$ \\
\hline \multirow{11}{*}{ Subtypes } & Meningothelial & $14(35.0)$ \\
\hline & Transitional & $12(30.0)$ \\
\hline & Atypical & $4(10.0)$ \\
\hline & Cystic meningioma & $2(5.0)$ \\
\hline & Nonspecific grade 1 & $2(5.0)$ \\
\hline & Angiomatosis & $2(5.0)$ \\
\hline & Psammomatous & $1(2.5)$ \\
\hline & Microcystic & $2(5.0)$ \\
\hline & Distance from the superior sagittal sinus & $20(50)$ \\
\hline & Distance from the transverse sinus & $6(15)$ \\
\hline & Distance to cavernous sinus & $14(35)$ \\
\hline
\end{tabular}

NEUR SCIENCE

A reverse association was observed between the degree of proximity and the volume of bleeding $(\mathrm{P}=0.06)$.

Furthermore, the mass size was positively correlated with estimated blood loss volume $(\mathrm{P}=0.003)$; however, there was no significant correlation between the volume of bleeding and other parameters, including the degree of edema, the volume of mass, the site of the tumor in the brain, the type of tumor, and maximum connection to the dura (Table 2). The mean time of operation was strongly correlated with the rate of bleeding. $(\mathrm{P}=0.001)$. We detected no relationship between the rate of hemorrhage, and gender, age, a history of diabetes, a history of coronary disease, a history of antiplatelet use, as well as the mean level of serum hemoglobin, WBC, and platelet counts. However, the rate of bleeding was more expected in hypertensive, compared to normotensive patients (Table 3 ).

\section{Discussion}

Hemorrhage following brain tumor surgery adversely affects the treatment outcome of such tumors. Accordingly, the volume of massive hemorrhage and the high need for transfusion during and after surgery is essential predictors of adverse outcomes in the treatment of these patients. Therefore, predicting this volume of surgical 
Table 2. The relationship between bleeding volume and imaging indices

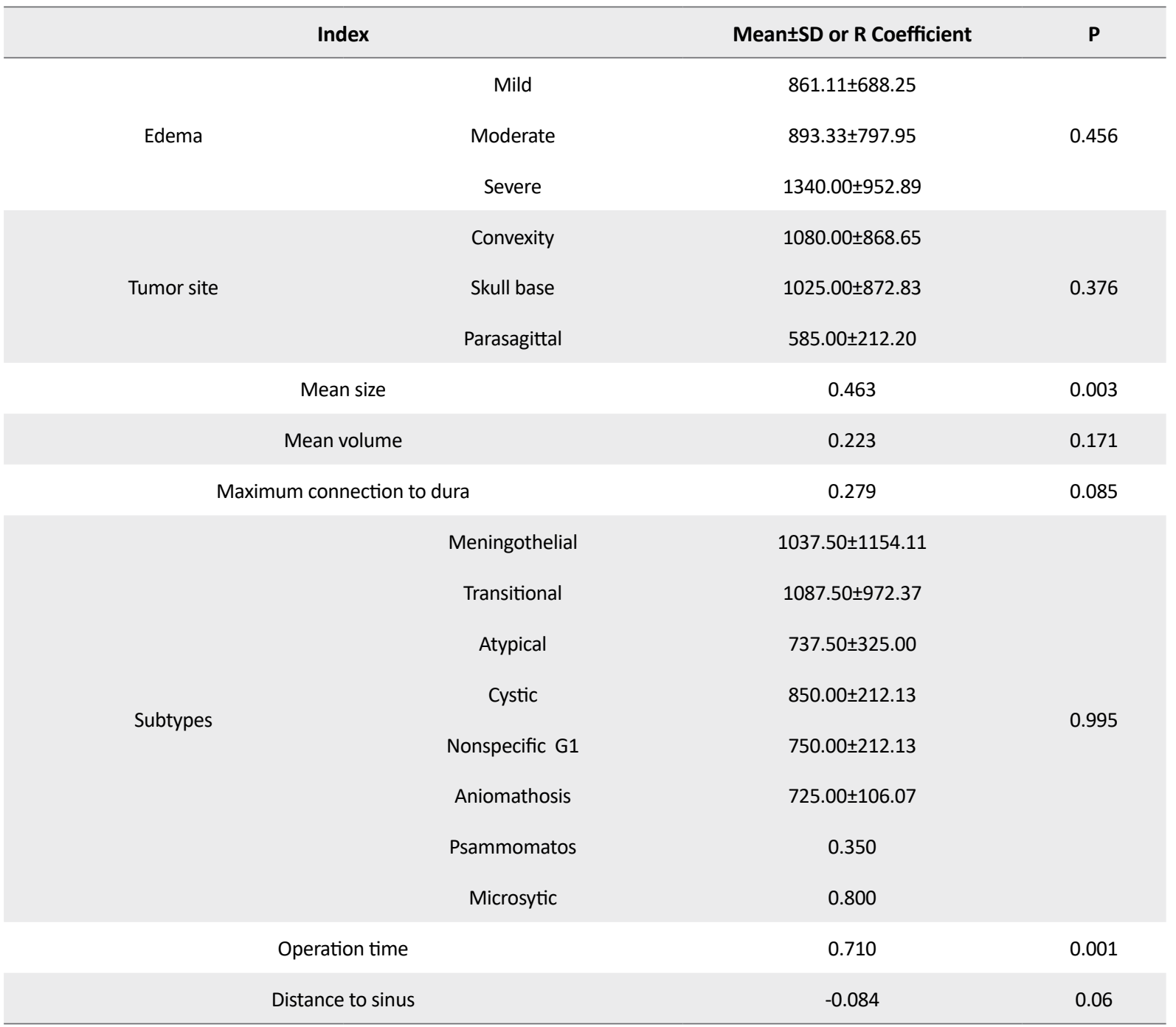

NEUR SCIENCE

bleeding can improve the patient's treatment outcomes and affect their long-term survival. Meningioma-induced Hemorrhage as well as bleeding from the surgical site are relatively common phenomena. Therefore, given its significant impact on the treatment outcome, its prediction is vital. The non-invasive evaluation of these patients before and after surgery by imaging, especially with MRI, is essential for assessing tumor progression and the favorable advancement of treatment; pre-surgical MRI indexes can be used to predict bleeding during surgery.

The main purpose of the current project was to describe the imaging characteristics of meningioma by MRI and aided to reduce intraoperative blood loss. In addition, we found that three of the indexes were related to the primary indicators of patients (history of hypertension), surgery (operation time), and the lesion's characteristics (tumor size); i.e. closely related to the volume of bleed- ing during the operation. In other words, in hypertensive patients or patients with large size of the meningioma, as well as a longer duration of surgery, this can increase the odds of bleeding during surgery. There was no significant correlation between the volume of bleeding and tumor volume. The reason for this finding cannot be easily evaluated. Perhaps, the largest tumor diameters are a better benchmark for presenting the depth of its penetration into the brain. For example, in two tumors of equal volume, in elliptical and spherical form, in the elliptical tumor, the shape of the penetration of the lesion is larger in the brain; thus, it takes more time to rescue such patients. Of course, this argument cannot be presented without a closer examination, and further studies are recommended in this area. 
Table 3. The relationship between bleeding volume and the baseline indices of patients

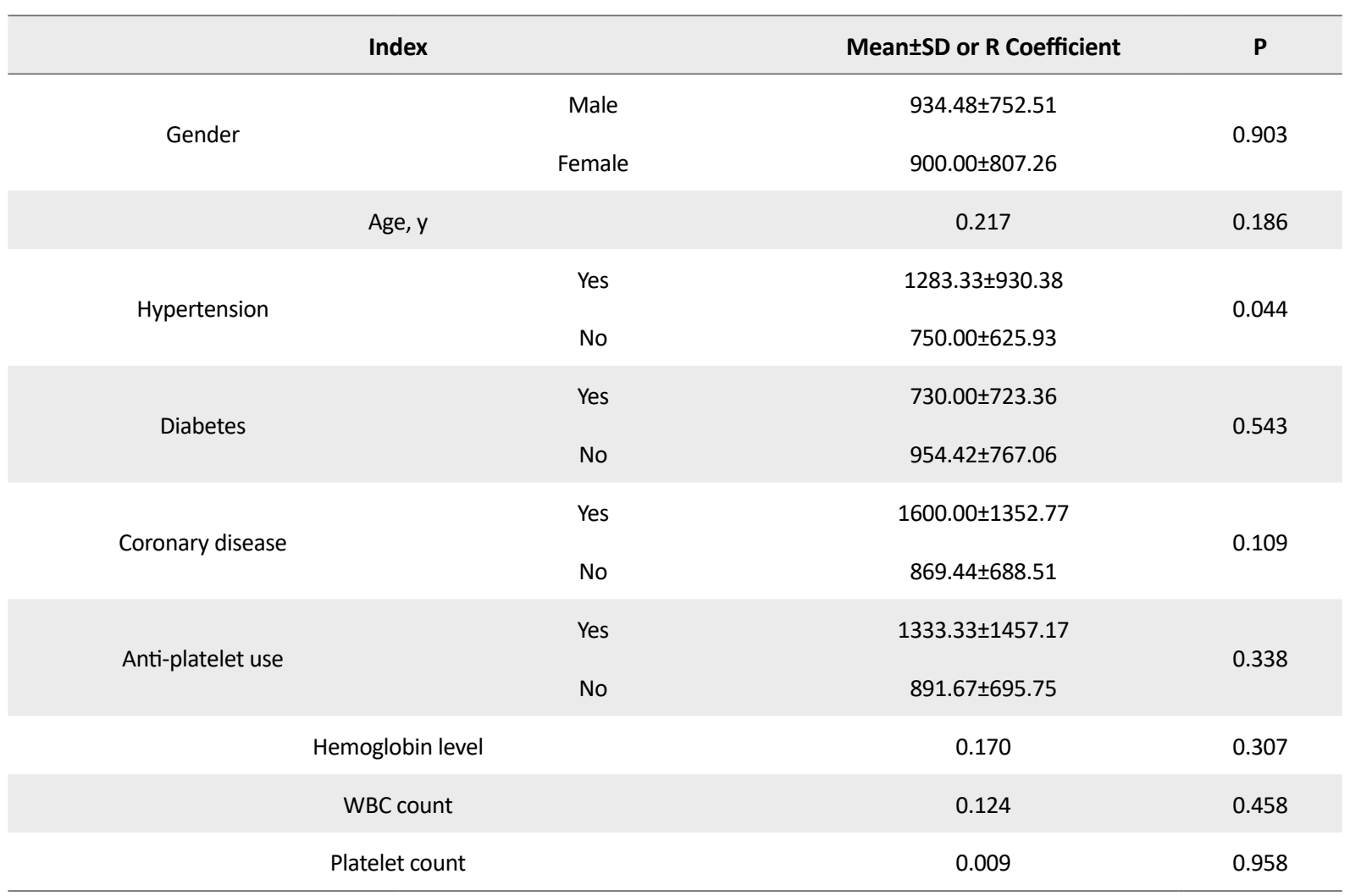

NEUR SCIENCE

Therefore, all measures should be taken to prevent bleeding and transfusion during surgery in hypertensive patients, as well as those with large meningioma.

It can be concluded that the closer tumor to the sinuses, the more blood volume will be lost during operation.

Data on the relationship between underlying characteristics and MRI and the prediction of bleeding during surgery are scarce. In a study (Lü, 2013), the related factors influencing bleeding severity were the origin of the tumor, tumor volume, bleeding vessel or venous sinus involvement, and the tumor size; these data were consistent with our study findings. They also argued that calcification, invasive behavior, dural tail symptoms, peripheral edema, and adjacent bone involvement did not affect hemorrhage. However, they disregarded evaluating the relationship between bleeding and baseline indices, like a history of hypertension. Moreover, Murph et al. (2013) found that preoperative MR-elastography can help determine the tumor's stiffness and bleeding during meningioma surgery. Hoover et al. mentioned that preoperative MRI could predict the tumor's stiffness and its bleeding probability in $90 \%$ of cases (Hoover, Morris \& Meyer, 2011). As previously mentioned, the cause of bleeding in meningeal tumors, either prematurely due to the nature of the tumor or during its therapeutic operation, can be attributed to the weakening of the nerve and ductile vessels, intratumoral angiogenesis, vascular wall invasion, blood dyscrasia, and simultaneous anticoagulation. However, the surgeon's experience should also be considered as the main cause of bleeding or its control (Lü, 2013).

Other studies investigated the association between tumor volume and hemorrhage during surgery, especially in the T2 sequence. In this study, we attempted to apply other imaging factors, i.e. less studied on them. However, the limitations of this study consisted of the lack of considering further factors in imaging in assessing the volume of bleeding. The other research limitation was the small sample size. We hope that in the future, these deficits will be compensated for achieving better results.

\section{Conclusion}

Bleeding in various volumes could be a common finding in intracranial meningioma surgery. In total, tumor size, the duration of surgery, a history of hypertension, and distance to the nearest sinuses were the main determinants for the severity of hemorrhage in patients undergoing meningioma surgery. 


\section{Ethical Considerations}

\section{Compliance with ethical guidelines}

All ethical principles were considered in this article. The participants were informed about the purpose of the research and its implementation stages; they were also assured about the confidentiality of their information; Moreover, They were allowed to leave the study whenever they wish, and if desired, the results of the research would be available to them; also this study was approved by the Ethics Committee of Iran University of Medical Sciences, Tehran, Iran (Code: 1396.9211255005).

\section{Funding}

The present study was extracted from the residency thesis of Ahmad Alagha at Iran University of Medical Sciences, Tehran.

\section{Authors' contributions}

All authors were equally contributed in preparing this article.

\section{Conflict of interest}

The authors declared no conflicts of interest.

Acknowledgments

The authors would like to appreciate all study participants as well as their families and hospital staff who contributed to the present research.

\section{References}

Toh, C. H., Castillo, M., Wong, A. C., Wei, K. C., Wong, H. F., \& Ng, S. H., et al. (2008). Differentiation between classic and atypical meningiomas with use of diffusion tensor imaging. American Journal of Neuroradiology, 29(9), 1630-5. [DOI:10.3174/ajnr.A1170] [PMID]

Whittle, I. R., Smith, C., Navoo, P., \& Collie, D. (2004). Meningiomas. The Lancet, 363(9420),1535-43. [DOI:10.1016/S01406736(04)16153-9]

O'leary, S., Adams, W. M., Parrish, R. W., \& Mukonoweshuro, W. (2007). Atypical imaging appearances of intracranial meningiomas. Clinical Radiology, 62(1), 10-17. [DOI:10.1016/j. crad.2006.09.009] [PMID]

Nagar, V. A., Ye, J. R., Ng, W. H., Chan, Y. H., Hui, F., \& Lee, C. K., et al. (2008). Diffusion-weighted MR imaging: Diagnosing atypical or malignant meningiomas and detecting tumor dedifferentiation. American Journal of Neuroradiology, 29(6) 1147-52. [DOI:10.3174/ajnr.A0996] [PMID]

Buetow, M. P., Buetow, P. C., \& Smirniotopoulos, J. G. (1991) Typical, atypical, and misleading features in meningioma. Radiographics, 11(6), 1087-06. [DOI:10.1148/radiographics.11.6.1749851] [PMID]

Tokgoz, N., Oner, Y. A., Kaymaz, M., Ucar, M., Yilmaz, G., \& Tali, T. E. (2005). Primary intraosseous meningioma: CT and MRI appearance. American Journal of Neuroradiology, 26(8), 2053-6. http://www.ajnr.org/content/ajnr/26/8/2053.full.pdf

Paek, S. H., Kim, S. H., Chang, K. H., Park, C. K., Kim, J. E., \& Kim, D. G., et al. (2005). Microcystic meningiomas: Radiological characteristics of 16 cases. Acta Neurochirurgica (Wien) 147(9), 965-72. [DOI:10.1007/s00701-005-0578-3] [PMID]

Hakyemez, B., Yildirim, N., Erdoðan, C., Kocaeli, H., Korfali, E., \& Parlak, M. (2006). Meningiomas with conventional MRI findings resembling intraaxial tumors: jan perfusion-weighted MRI be helpful in differentiation? Neuroradiology, 48(10), 695-702. [DOI:10.1007/s00234-006-0115-y] [PMID]

Elder, J. B., Atkinson, R., Zee, C. S., \& Chen, T. C. (2007). Primary intraosseous meningioma. Neurosurgical Focus, 23(4), 1-9. [DOI:10.3171/FOC-07/10/E13] [PMID]

Komotar, R. J., Keswani, S. C., \& Wityk, R. J. (2003). Meningioma presenting as stroke: report of two cases and estimation of incidence. Journal of Neurology, Neurosurgery \& Psychiatry, 74(1), 136-7. https://jnnp.bmj.com/content/74/1/136.short

Heye, S., Maleux, G., Van Loon, J., \& Wilms, G. (2006). Symptomatic stenosis of the cavernous portion of the internal carotid artery due to an irresectable medial sphenoid wing meningioma: treatment by endovascular stent placement. American journal of neuroradiology, 27(7), 1532-4. http://www.ajnr. org/content/27/7/1532.short

Yue, Q., Isobe, T., Shibata, Y., Anno, I., Kawamura, H., \& Yamamoto, Y., et al. (2008). New observations concerning the interpretation of magnetic resonance spectroscopy of meningioma. European Radiology, 18(12), 2901. [DOI:10.1007/ s00330-008-1079-6] [PMID]

Chen, T. Y., Lai, P. H., Ho, J. T., Wang, J. S., Chen, W. L., \& Pan H. B., et al. (2004). Magnetic resonance imaging and diffusionweighted images of cystic meningioma: Correlating with histopathology. Clinical Imaging, 28(1), 10-19. [DOI:10.1016/ S0899-7071(03)00032-9

Bitzer, M., Opitz, H., Popp, J., Morgalla, M., Gruber, A., Heiss, E., \& Voigt, K. (1998). Angiogenesis and brain oedema in intracranial meningiomas: influence of vascular endothelial growth factor. Acta Neurochirurgica, 140(4), 333-40. [DOI:10.1007/ s007010050106] [PMID]

Nakano, T., Asano, K., Miura, H., Itoh, S., \& Suzuki, S. (2002). Meningiomas with brain edema: radiological characteristics on MRI and review of the literature. Clinical Imaging, 26(4), 243-9. [DOI:10.1016/S0899-7071(02)00433-3]

Lü, J. (2013). Correlation between preoperative imaging features and intraoperative blood loss of meningioma: a new scoring system for predicting intraoperative blood loss. Journal of Neurosurgical Sciences, 57(2), 153-61. https://europepmc.org/ article/med $/ 23676863$ 
Hoover, J. M., Morris, J. M., \& Meyer, F. B. (2011). Use of preoperative magnetic resonance imaging T1 and T2 sequences to determine intraoperative meningioma consistency. Surgical Neurology International, 2, 142. [DOI:10.4103/2152-7806.85983] [PMID] [PMCID] 


\title{
Research Paper: The Relationship Between Ruminating the Catastrophic Consequences of Bodily Changes and Positive Reappraisal and Practical Problem-Solving Strategies in Individuals With Illness Anxiety Disorder
}

\author{
Mina Elhamias ${ }^{* *}$ (D), Mohsen Dehghani ${ }^{1}$, Mahmood Heidari ${ }^{1}$, Ali Khatibi² ${ }^{\mathbb{D}}$ \\ 1. Department of Psychology, Faculty of Education and Psychology, Shahid Beheshti University, Tehran, Iran \\ 2. Interdisciplinary Program in Neuroscience, Bilkent University, Ankara, Turkey.
}

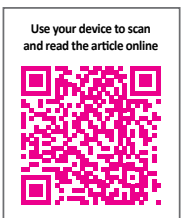

Cftation: Elhamiasl, M., Dehghani, M., Heidari, M., \& Khatibi, A. (2020). The Relationship Between Ruminating the Catastrophic Consequences of Bodily Changes and Positive Reappraisal and Practical Problem-Solving Strategies in Individuals With Illness Anxiety Disorder. Basic and Clinical Neuroscience, 11(5), 639-648. http://dx.doi.org/10.32598/bcn.9.10.240

ol http://dx.doi.org/10.32598/bcn.9.10.240

\section{Article info:}

Received: 12 May 2019

First Revision: 22 May 2019

Accepted: 05 Nov 2019

Available Online: 01 Sep 2020

Keywords:

Illness anxiety disorder, Emotion regulation, Interpretation bias, Catastrophizing, Bodily symptoms

\begin{abstract}
A B S T RA C T
Introduction: Cognitive emotion regulation is suggested to contribute to Illness Anxiety Disorder (IAD). Reappraisal and suppression are essential ER strategies with controversial data about their roles in IAD. Relevant studies are mostly limited to exploring these two strategies in individuals without such disorder. Therefore, we aimed to study the role of emotion regulation in the psychopathology of IAD by evaluating other ER strategies in illnessanxious individuals. Furthermore, we investigated the relationship between IAD and emotion regulation by targeting the role of interpretation bias for health-related information.

Methods: The study participants were 60 university students. They underwent a semistructured clinical interview to assess the presence or absence of IAD symptoms $(n=30 /$ group). They completed a battery of questionnaires measuring IAD, emotion regulation, and interpretation bias.

Results: The illness-anxious group applied significantly less reappraisal and refocus on planning and more rumination, catastrophizing, and acceptance strategies, compared to the controls. Besides, interpretation bias was positively correlated with rumination and catastrophizing; while its association with reappraisal and planning was negative.

Conclusion: Both functional (e.g. reappraisal \& planning) and dysfunctional strategies (e.g. rumination \& catastrophizing) contributed to the psychopathology of IAD. The biased interpretation of bodily information could make individuals prone to ruminate about the catastrophic consequences of bodily changes; such conditions interrupt fostering more positive reappraisal or practical problem-solving strategies.
\end{abstract}




\section{Highlights}

- The illness anxiety disorder group used different emotion regulation strategies, compared to the controls.

- The illness anxiety disorder group used less reappraisal and planning than the controls.

- The illness anxiety disorder group applied more dysfunctional emotion regulation strategies, compared to the controls.

- Interpretation bias was positively correlated with rumination and catastrophizing.

- Interpretation bias was negatively associated with reappraisal and planning.

\section{Plain Language Summary}

Worries about our health could help to detect a possible physical problem and to treat it before getting serious. However, severe preoccupation with the fear of severe illnesses can lead to excessive anxiety levels, known as illness anxiety disorder, that can interrupt daily life. Therefore, it is essential to understand which factors might make people prone to unnecessary worrying about bodily sensations. Impaired emotion regulation abilities, which refers to the strategies involved in down-regulating negative emotions, might be one of these factors. We studied if people with illness anxiety disorder might use different emotion regulation strategies, compared to those without illness anxiety. Besides, interpretation bias for health-related information (i.e. expecting the worse outcome for a health-threatening situation) is the main feature of illness anxiety. Therefore, we tested if there is a relationship between interpretation bias and emotion regulation in people with and without the illness anxiety disorder. Our results revealed that people with illness anxiety disorder used fewer appropriate emotion regulation strategies, such as reappraisal and planning, compared to those without such disorder. Illnessanxious individuals also showed higher rumination and catastrophizing, compared to the healthy ones. Our results also suggested that increases in interpretation bias were associated with enhanced rumination and catastrophizing and decreased functional reappraisal and planning. Therefore, individuals' inability to find alternative positive outcomes for a situation might make them think constantly about that situation. Passive catastrophic thinking, in addition, prevents people from engaging in more active solutions, such as planning to solve their health problems with more appropriate solutions. These results can be used to educate illness-anxious people to stop rumination about catastrophic interpretations and replace them with safer appraisals and plans to reduce their anxiety.

\section{Introduction}

I

llness Anxiety Disorder (IAD) refers to preoccupation with the fear of a serious illness that increases anxiety level and makes individuals vigilant to their health state (American Psychiatry Association, 2013). IAD can be considered as an independent disorder; however, it is a prevalent symptom of other anxiety disorders, such as Generalized Anxiety Disorder (GAD), specific phobia, and Obsessive-Compulsive Disorder (OCD) (See Abramowitz, Brigidi \& Foa, 1999; Deacon \& Abramowita, 2008). The prevalence of IAD and its associated frequent medical visits, as a dramatic burden on the healthcare system, demand a better understanding of the problem (Barsky, Ettner, Horsky \& Bates, 2001; Gorgen, Hiller \& Witthoft, 2014).
Emotion Regulation (ER) is a model that might increase our comprehension of IAD. Emotion dysregulation has been reported among the most essential psychological construct observable in approximately $75 \%$ of psychiatric disorders; it leads to experiencing negative emotions severely and uncontrollably, as they lack the required skills for managing and regulating their severe emotions (Campbell-Sills, Ellard \& Barlow, 2013). Gross and John (2003) suggests that cognitive reappraisal and expressive suppression are two major ER strategies. Cognitive reappraisal refers to the reinterpretation of an emotional event to change the meaning of that event and related emotions. Expressive suppression is an ER strategy to hide, control, or modify emotions and emotional behaviors (Gross \& John, 2003; Gross \& Levenson, 1993). Reappraisal is an appropriate method leading to the reduction of negative emotions, such as anxiety and depression; however, suppression is a maladaptive one associated with increased negative emotions in clinical 
and non-clinical samples (Koster, Rassin, Crombex, \& Naring., 2003; Gross \& Levenson, 1993; Gorgen et al., 2014). Previous studies suggested that suppression and reappraisal might have respectively positive and negative relationships with worry about health and preoccupation with somatic sensations (Bardeen \& Fergus, 2014). However, others have claimed that IAD and reappraisal are not related; they proposed that psychopathology is not related to functional strategies, like reappraisal. Gorgen et al. (2014) reported that disease phobia and disease conviction were not correlated with reappraisal. Additionally, in Fergus and Valentiner's study (2010), reappraisal was only related to the perceptual aspect of hypochondria (as sensitivity to innocuous bodily sensations), rather than the affective, behavioral, or cognitive ones. These studies indicated that dysfunctional ER strategies, like suppression, were involved in IAD and its components, including body vigilance. However, another study reported that none of these strategies were related to IAD (Gerolimatos \& Edelstein, 2012).

Furthermore, the literature on the relationship between interpretation bias for health-related information suggested as the main component of IAD, and ER strategies could be considered as supplementary evidence supporting the role of ER pathology in the development and maintenance of IAD. However, some studies are based on data on hypochondria rather than IAD; it is a novel diagnostic term replacing hypochondria with some features in common (e.g. fear of illness). Interpretation bias refers to the attribution of catastrophic meaning to ambiguous and threatening information. Based on this definition, individuals with hypochondriasis as well as those with IAD, compared to healthy groups, may consider more negative consequences when appraising health-related situations. These negative thoughts are expressed in the form of most catastrophic appraisals; in turn, they increase the perception of bodily sensations leading to a more catastrophic appraisal of health-related issues. Such a positive relationship between negative appraisal and the biased interpretation of health-threatening data has been supported by several studies (Haenen, de Jong, Schmidt, Stevens \& Visser, 2000; Weck, Neng, Richtberg \& Stangier, 2012). These data suggested that positive reappraisal, as an ER mechanism that enables individuals in selecting safer interpretations, might be an impaired strategy in IAD.

Additionally, by considering the role of suppression, individuals with IAD avoid situations that might activate illness schemas. This can occur by distracting or suppressing biased interpretations and undesired emotions. Applying suppression could make individuals ex- perience unwanted bodily sensations, such as pain and catastrophic thoughts more chronically; it prevents them from coping with the underpinning emotion (Cioffi \& Holloway, 1993; Gilliam et al., 2010; Wegner, Schneider, Carter \& White, 1987). IAD refers to the chronic experience of worries about illness-related sensations and catastrophic interpretations of them; accordingly, suppression might be considered among the involved mechanisms in such chronicity. Therefore, concerning the relationship between interpretation bias and ER strategies, both strategies could be involved in the maintenance of IAD.

Considering the above-mentioned inconsistent research findings on the relationship between IAD and ER strategies of reappraisal and suppression, a potential cause of data discrepancy might be the mere focus on populations without IAD. Comparing ER strategies between individuals with higher and lower levels of illness anxiety could endorse if a difference in ER is a possible maintenance factor in IAD. another issue concerning studies on ER and IAD was only measuring reappraisal and suppression. However, some other ER strategies might prone individuals vulnerable to IAD. Finding these strategies might help us in the better conceptualization of the role of ER strategies in IAD.

Accordingly, the current study aimed to examine ER strategies in two IAD and control groups. We hypothesized that while reappraisal, as a functional strategy, would be used more by the control group, in comparison to the IAD group subjects, the IAD group would apply a more dysfunctional strategy of suppression, compared to the controls. In addition, individuals with IAD, compared to the controls, would use other functional strategies, such as acceptance and refocus on planning less; while would apply other dysfunctional strategies, such as rumination and catastrophizing more. Then, to explore the effect of ER in IAD, we evaluated the relationship between ER strategies and interpretation bias for health-related information. We assumed that higher interpretation bias would be related to less functional strategies and more dysfunctional ones.

\section{Materials and Methods}

The IAD group samples were 30 students from Shahid Beheshti University; they were selected through a research participation enouncement requesting individuals with IAD symptoms. In this announcement, some of the IAD symptoms, including experiencing worries about health, checking body status, avoidance from healthrelated information or searching for such data, and being 
sensitive to bodily changes, were listed. Volunteer students were supposed to inform the experimenter using email or text. To confirm the presence of IAD symptoms, one of the authors (M.E.; MA in Clinical Psychology; 5 years of supervised \& independent practice in anxiety disorders) assessed the IAD symptoms in volunteers by a semi-structured interview. A serious medical condition, receiving psychiatric medications, and the diagnosis of any other psychiatric disorders, such as major depressive disorder, obsessive-compulsive disorder, panic disorder, and psychosis in the last 5 years were the research exclusion criteria. Considering the inclusion and the exclusion criteria of the study, 30 participants (15 females) were selected from 47 volunteers. Other 17 participants were excluded due to not meeting the study inclusion criteria for IAD (4); medical diseases (3); a history of or current episodes of another psychiatric disorder (6); using psychiatric medications (2), and being under psychotherapy due to dysfunctional IAD (2). Overall, none of the selected participants experienced dysfunctional IAD that affected their daily living activities. They also had no dysfunctional comorbid psychiatric disorder, such as major depressive disorder, obsessive-compulsive disorder, or psychosis. Three of the research participants reported experiencing worries in other areas, including education or personal life. However, such experiences were not dysfunctional enough to visit a psychologist or receive a diagnosis of Generalized Anxiety Disorder (GAD). The volunteers who were excluded from the study due to experiencing psychiatric disorders were given some clarifying information on their observed problem; they were suggested to visit a psychologist at the psychotherapy and counseling center of Shahid Beheshti University.

The control group members included 30 students from Shahid Beheshti University. They were selected through an announcement requesting individuals without IAD symptoms. Volunteer students were supposed to inform the experimenter using email or text. Volunteers whose gender and age range matched to the IAD group were invited for an interview. To clarify the absence of IAD symptoms, a semi-structured interview, assessing illness anxiety symptoms, was conducted by a clinical psychologist. Encountering a serious medical condition, being under medical or psychiatric medications, and experiencing other psychiatric disorders were the exclusion criteria for this group. Thirty participants (15 females) among 37 volunteers were determined as eligible to be included in the control group. Eight volunteers were excluded due to experiencing medical diseases (2); a history or presence of another psychiatric disorder (2); using psychiatric medications (2), and a recent history of surgery (1). However, one of the thirty volunteers could not attend the measurement session due to an unexpected medical problem.

\subsection{The following tools were used in the present re- search}

The Structured Clinical Interview for DSM-5, Clinician Version (SCID-5-CV; First, Williams, Karg, \& Spitzer, 2015): SCID-5-CV is a semi-structured interview guide to make a diagnosis based on the Diagnostic and Statistical Manual of Mental Disorders, 5th Edition (DSM-5) criteria. The Persian version of this manual was administered by the author (M.E) who holds a certified degree in Clinical Psychology, to check the existence and absence of IAD symptoms, respectively in the test and control groups.

Short Health Anxiety Inventory (SHAI; Salkovskis, Rimes, \& Warwick, 2002): SHAI is an 18-item 4-point Likert-type questionnaire that evaluates IAD, independent from physical health status. These items measure various aspects of IAD, such as health worries, the awareness of bodily sensations and changes, and the negative consequents of illnesses. This inventory has been reported to have appropriate validity and reliability (Salkovskis et al., 2002). The Persian version of this questionnaire was used in the current study. The Internal consistency of the Persian version of this questionnaire in the current study, calculated by Cronbach's alpha coefficient, was equal to 0.93 .

Whiteley Index (WI): WI is a 14-item inventory, targeting IAD. It is scored dichotomously and continuously; we used the continuous version of scoring in the current study. This measure has a strong convergent correlation $(\mathrm{r}=0.80)$ with other scales of IAD (Pilowsky, 1967). The Persian version of this questionnaire was used in the current study. The Internal consistency of the Persian version of this scale in the current study, calculated by Cronbach's alpha coefficient, equaled 0.94.

Cognitions About Body and Health Questionnaire (CABAH): CABAH is a 31-item 5-point Likert-type questionnaire, assessing 5 subscales of catastrophizing interpretation of bodily complains, autonomic sensations, bodily weakness, the intolerance of bodily complains, and health habits (Rief, Hiller, \& Margraf, 1998). The internal consistency of this scale in clinical and healthy populations was reported as 0.90 and 0.80 , respectively. The Persian version of this questionnaire was used in the current study. The internal consistency of the Persian version of this inventory in the current study was 
calculated by Cronbach's alpha coefficient as 0.93 . In the current study, we only used the catastrophizing interpretation of bodily complaints subscale as the index of interpretation bias toward health-related information.

Emotion Regulation Questionnaire (ERQ): ERQ is a 10 -item scale designed to explore cognitive reappraisal and expressive suppression. The original internal consistency of the questionnaire was reported as appropriate (Gross \& John, 2003). The Persian version of this scale was used in the current study. The internal consistency of the Persian version of this questionnaire in the current study, as calculated by Cronbach's alpha coefficient, for reappraisal and suppression subscales was 0.84 and 0.82 , respectively.

Cognitive emotion regulation questionnaire (CERQ): CERQ is a 36-item questionnaire that measures cognitive ER strategies in response to a stressful life event. Its subscales demonstrated acceptable internal consistency (Cronbach's $\alpha>0.70$ ) (Garnefski, Kraaij \& Spinhoven, 2001). The Persian version of this inventory was used in the current study. The internal consistency of the Persian version of the tool's subscales was calculated by Cronbach's alpha coefficient to range between 0.74 and 0.86 .

The present study was approved by the Ethics Committee of the Psychology Department at Shahid Beheshti University (Code: 30514). All study participants have read and signed the informed consent form at the beginning of the first session (interview session). The consent form contained information about the study purposes (individual differences in some psychological factors), the measurement methods, the associated risks and benefits, and the rules for withdrawal from the study. For recruiting the study participants with IAD, volunteers were called by advertising in public places at the university. Upon the expression of interest, not meeting the exclusion criteria, as well as considering equal gender ratio, they were invited to an interview; during which, they were individually interviewed by a clinical psychologist to examine the presence of IAD, according to the semistructured clinical interview. Those volunteers who met the IAD symptoms were selected as the IAD group samples. The subjects were informed by the experimenter that the current study consisted of conducting some questionnaires, evaluating individual differences in some psychological factors. Then, the date for the second session was set for them to meet the experimenter at the test lab for further measurements. In the second session, they were requested to complete the questionnaires after reading the instructions. After completing the test battery, the research participants were debriefed and the session was terminated. Following completing the experiment in the IAD group, volunteers for the control group were called by advertising in the public places of the University. Upon the expression of interest, being matched to the age range and gender of the IAD group, and not meeting the exclusion criteria, they were invited to an interview; during which, they were individually interviewed by a clinical psychologist to examine the absence of IDA according to the semi-structured clinical interview. Those who did not meet the IAD symptoms were selected as the controls. The procedures for signing a consent form, interview, and collecting data were the same as those for the IAD group.

\section{Results}

Before conducting the major statistical analysis, the data belonging to 3 participants ( 2 in the IAD group) were removed due to the high number of missing data. The descriptive statistics on 56 research participants' data indicated an equal gender ratio in the whole sample (28 females). Gender distribution in the IAD and control groups was close to each other, as well (15 females in the IAD group \& 13 female controls). the Independent Samples t-test data revealed no significant difference between male (Mean $\pm \mathrm{SD}=19.82 \pm 10.44) ; \mathrm{t}_{54}=-0.21, \mathrm{P}=0.83$ ) and female $\left.\mathrm{s}(\mathrm{Mean} \pm \mathrm{SD}=20.42 \pm 10.98) ; \mathrm{t}_{54}=-0.21, \mathrm{P}=0.83\right)$ samples in health anxiety levels, assessed by HAI. Male (Mean $\pm \mathrm{SD}=22 \pm 10.97) ; \mathrm{t}_{54}=-0.23, \mathrm{P}=0.81$ ) and female $\left(\mathrm{Mean} \pm \mathrm{SD}=22.71 \pm 11.87\right.$ ); $\mathrm{t}_{54}=-0.23, \mathrm{P}=0.81$ ) difference in health anxiety level measured by WI score was insignificant as well. The study groups matched according to age as well (health-anxious group: $\mathrm{Mean} \pm \mathrm{SD}=23.17 \pm 2.38$; controls: Mean $\pm \mathrm{SD}=23.82 \pm 2.61$ ).

To examine our research hypothesis about the lower levels of functional and higher levels of dysfunctional ER strategies in the IAD group, compared with the controls, we performed a Multivariate Analysis of Variance (MANOVA) while the subscales of ERQ, i.e. reappraisal and suppression, as well as the subscales of CERQ, i.e. positive reappraisal, refocus on planning, catastrophizing, rumination, acceptance, positive focusing, putting into perspective, self-blame, and other-blame was considered as the dependent variables and group were the fixed one.

The equality of covariance and error variance, as two main prerequisites of MANOVA, were assessed using Box's M and Levene's tests. The Box's M test result was not significant $(\mathrm{P}=0.51)$; therefore, the MANOVA assumption of homogeneity of covariance was approved. The Levene's test data for each dependent variable were 
Table 1. The results of descriptive statistics tests and MANOVA for between-group differences in ER strategies ${ }^{1}(n=29)$

\begin{tabular}{|c|c|c|c|c|c|}
\hline \multirow{2}{*}{ Variabels } & \multicolumn{2}{|c|}{ Mean $\pm S D$} & \multirow{2}{*}{ SS } & \multirow{2}{*}{ MS } & \multirow{2}{*}{$\mathbf{F}$} \\
\hline & IAD & Control & & & \\
\hline Reappraisal & $23.28 \pm 4.95$ & $27.78 \pm 5.04$ & 283.50 & 283.50 & 11.53 \\
\hline Suppression & $13.64 \pm 5.37$ & $14.78 \pm 4.60$ & 18.28 & 18.28 & 0.73 \\
\hline Positive reappraisal & $12.71 \pm 3.14$ & $15.10 \pm 3.40$ & 80.16 & 80.16 & 7.45 \\
\hline Refocus on planning & $13.92 \pm 2.82$ & $15.75 \pm 2.84$ & 46.44 & 46.44 & 5.76 \\
\hline Catastrophizing & $12.53 \pm 2.80$ & $9.75 \pm 3.40$ & 108.64 & 108.64 & 11.14 \\
\hline Rumination & $15.14 \pm 2.66$ & $11.50 \pm 3.38$ & 185.78 & 185.78 & 20.04 \\
\hline Acceptance & $14.32 \pm 2.49$ & $13.03 \pm 1.97$ & 23.14 & 23.14 & 4.57 \\
\hline Positive focusing & $12.50 \pm 2.44$ & $13.39 \pm 2.93$ & 11.16 & 11.16 & 1.53 \\
\hline Put into perspective & $12.92 \pm 2.85$ & $12.10 \pm 2.71$ & 9.44 & 9.44 & 1.21 \\
\hline Self-blame & $13.82 \pm 2.93$ & $13.50 \pm 2.84$ & 1.44 & 1.44 & 0.17 \\
\hline Other-blame & $9.46 \pm 3.58$ & $9.78 \pm 2.89$ & 1.44 & 1.44 & 0.13 \\
\hline
\end{tabular}

SD: Standard Deviation, SS: Sum of Squares; MS: Mean of Squares;

NEURSCIENCE

${ }^{1}$ The results of descriptive statistics and MANOVA for reappraisal, suppression, positive reappraisal, refocus on planning, catastrophizing, rumination, acceptance, positive focusing, put into perspective, self-blame, and other-blame in IAD and control groups.

insignificant ( $\mathrm{P}>0.05)$; accordingly, the error variance of all the variables was equal across two research groups. MANOVA revealed a significant multivariate main effect for ER strategy [Pillai's trace $=0.505, \mathrm{~F}_{11,44}=4.07$, $\mathrm{P}=0.001, \eta \mathrm{p}^{2}=0.50$, observed power $\left.=0.99\right]$. According to the univariate test data, the IAD group, compared to the controls, reported significantly lower levels of reappraisal $\left[\mathrm{F}_{1,5}=11.35, \mathrm{P}=0.001, \eta \mathrm{p}^{2}=0.17\right]$, positive reappraisal $\left[\mathrm{F}_{1,54}=7.45, \mathrm{P}=0.009, \eta \mathrm{p}^{2}=0.12\right]$, and refocus on planning $\left[\mathrm{F}_{1,54}=5.76, \mathrm{P}=0.02, \eta \mathrm{p}^{2}=0.09\right]$, but higher levels of acceptance $\left[\mathrm{F}_{1,54}=4.57, \mathrm{P}=0.03, \eta \mathrm{p}^{2}=0.07\right]$, rumination $\left[\mathrm{F}_{1,54}=20.04, \mathrm{P}=0.001, \eta \mathrm{p}^{2}=0.27\right]$, and catastrophizing $\left[\mathrm{F}_{1,54}=11.14, \mathrm{P}=0.002, \eta \mathrm{p}^{2}=0.17\right]$. The between-group differences were not significant for the other ER strategies. The relevant results are presented in Table 1.

To explore the specific relationship between IAD and each ER strategy, we used Pearson correlation analysis to evaluate the correlation between two illness anxiety indexes (i.e. HAI \& WI) and ER strategies (assessed by ERQ \& CERQ) in the total sample $(\mathrm{N}=56)$. Consistent with the t-test findings, the levels of IAD in HAI were negatively correlated with applying reappraisal $(\mathrm{r}=-0.50$, $\mathrm{P}<0.001)$, positive reappraisal $(\mathrm{r}=-0.34, \mathrm{P}<0.01)$, and refocus on planning $(\mathrm{r}=-0.32, \mathrm{P}<0.05)$. Besides, the extent of IAD in WI was negatively correlated with reap- praisal $(\mathrm{r}=-0.50, \mathrm{P}<0.001)$, positive reappraisal $(\mathrm{r}=-0.42$, $\mathrm{P}<0.001)$, and refocus on planning $(\mathrm{r}=-0.42, \mathrm{P}<0.001)$. The levels of rumination $(\mathrm{r}=0.50, \mathrm{P}<0.001)$ and catastrophizing $(\mathrm{r}=0.38, \mathrm{P}<0.01)$ were positively associated with IAD scores in HAI. Scores in WI were also positively correlated with rumination $(\mathrm{r}=0.54, \mathrm{P}<0.001)$ and catastrophizing $(\mathrm{r}=0.44, \mathrm{P}<0.001)$. Table 2 lists these significant correlations.

Furthermore, we explored the relationship between ER and IAD by targeting interpretation bias. For this purpose, we used the Pearson correlation analysis to investigate the relationship between interpretation bias and ER strategies in the total sample. As hypothesized, interpretation bias was negatively correlated with reappraisal $(\mathrm{r}=-0.34, \mathrm{P}<0.01)$ and refocus on planning $(\mathrm{r}=-$ $0.30, \mathrm{P}<0.05$ ), and positively associated with rumination $(\mathrm{r}=0.44, \mathrm{P}<0.001)$ and catastrophizing $(\mathrm{r}=0.28, \mathrm{P}<0.05)$. However, no significant correlation was detected between interpretation bias and suppression. The significant correlations are reported in Table 3.

\section{Discussion}

In this study, we investigated whether ER can explain the differences between IAD and healthy control 
Table 2. The relationship between health anxiety and ER strategies ${ }^{1}$

\begin{tabular}{|c|c|c|c|c|c|}
\hline Variable & $\begin{array}{c}\text { ERQ } \\
\text { Reappraisal }\end{array}$ & $\begin{array}{c}\text { CERQ } \\
\text { Positive Reappraisal }\end{array}$ & $\begin{array}{c}\text { CERQ } \\
\text { Rumination }\end{array}$ & $\begin{array}{c}\text { CERQ } \\
\text { Catastrophizing }\end{array}$ & $\begin{array}{c}\text { CERQ } \\
\text { Refocus on Planning }\end{array}$ \\
\hline SHAI & $-0.50^{*}$ & $-0.34 * *$ & $0.50 *$ & $0.38^{* *}$ & $-0.32 * * *$ \\
\hline WI & $-0.50 *$ & $-0.42^{*}$ & $0.54 *$ & $0.44^{*}$ & $-0.42 *$ \\
\hline
\end{tabular}

${ }^{*} \mathrm{P}<0.05 ; * * \mathrm{P}<0.01 ; * * * \mathrm{P}<0.001$

NEUR:SCIENCE

SHAI: Short Health Anxiety Inventory; WI: Whiteley Index; ERQ: Emotion Regulation Questionnaire; CERQ: Cognitive Emotion Regulation Questionnaire;

${ }^{1}$ Correlations between health anxiety indices (Short Health Anxiety Inventory and Whiteley Index) and the subscales of ER strategies.

Table 3. The relationship between interpretation bias and ER strategies

\begin{tabular}{ccccc}
\hline Variable & $\begin{array}{c}\text { ERQ } \\
\text { Reappraisal }\end{array}$ & $\begin{array}{c}\text { CERQ } \\
\text { Rumination }\end{array}$ & $\begin{array}{c}\text { CERQ } \\
\text { Catastrophizing }\end{array}$ & $\begin{array}{c}\text { CERQ } \\
\text { Refocus on planning }\end{array}$ \\
\hline $\begin{array}{c}\text { CABAH } \\
\text { Interpretation bias }\end{array}$ & $-0.34^{*}$ & $0.44^{* * *}$ & $0.28^{* *}$ & $-0.30^{* *}$ \\
\hline$* * * \mathrm{P}<0.001 ; * * \mathrm{P}<0.05, * \mathrm{P}<0.01 ;$ & & & & NEUR:\%SCIENCE \\
\hline
\end{tabular}

ERQ: Emotion Regulation Questionnaire; CERQ: Cognitive Emotion Regulation Questionnaire; CABAH: Cognitions About Body And Health.

groups. We observed that individuals with IAD used less reappraisal, compared to the controls; however, there was no such difference in suppression. The IAD group used more acceptance, rumination, and catastrophizing strategies and less positive reappraisal and refocus on planning, compared to the controls. Greater functional strategies of reappraisal and refocus on planning were associated with less interpretation bias; while the dysfunctional strategies of catastrophizing and rumination were positively correlated with interpretation bias.

The observed difference between the IAD and control samples in employing reappraisal suggested that reappraisal could be related to the IAD pathology. Bardeen and Fergus (2014) argued that less application of reappraisal was accompanied by higher levels of health-related worries and preoccupation with bodily sensations. However, the groups were not different based on adopting suppression. There is considerable inconsistency in the literature about the influence of suppression in IAD. Some studies supported the relationship between suppression and IAD and its components, including bodily vigilance (Gorgen et ak., 2014; Fergus, \& Valentiner, 2010); however, some others did not support these findings (Gerolimatos, \& Edelstein, 2012). Suppression is a dysfunctional strategy in the Gross model; however, some studies revealed that the functionality or dysfunctionality of reappraisal and suppression depends on the context (Bardeen, \& Fergus, 2014). Therefore, both study group members might have used suppression, but its consequences depend on the context.

We also explored whether other ER strategies, addressed as involved in IAD, may contribute to the differences between individuals with and without IAD. We found that refocus on planning was higher applied by the control group, compared to the IAD one. Refocus on planning reflects an active coping strategy that makes individuals take practical measures to cope with the problem rather than ruminating about it (Garnefski et al., 2001). To our knowledge, no study has investigated this strategy in IAD; however, some researchers reported that higher refocus on planning was associated with encountering less negative emotions, such as depression and anxiety (Kulpa, Zietalewicz, Kosowics, StypulaCiuba \& Ziolkowska, 2016). Furthermore, the effect of reduced refocus on planning in IAD could be explained better by rumination strategy. In this study, rumination was significantly higher in individuals with IAD, in comparison to the controls. Rumination refers to a maladaptive problem-solving style that forces individuals to constantly think about negative emotions and their possible causes, instead of engaging in more practical solutions (Garnefski et al., 2001). Therefore, a greater focusing on repetitive dysfunctional thoughts (i.e. rumination) could be accompanied by less focusing on active planning. Supporting this inference, it was reported that dysphoric students with higher scores in rumination could implement their plans with less possibility, compared to those 
with less rumination (Lyubomirsky, Tucker, Caldwell \& Berg, 1999). This inference was supported when we detected a significant negative correlation between the refocus on planning and rumination. Catastrophizing was another cognitive strategy, i.e. significantly higher among the studied individuals with IAD. This finding supports the data that individuals preoccupied with health issues used to consider more catastrophic outcomes for illnesses, compared with the control group (Weck et al., 2012). Moreover, acceptance, as a cognitive ER strategy, was significantly used further by the IAD group, compared to the controls. Acceptance refers to a strategy that enables an individual to accept what has occurred and to believe that it cannot be changed (Garnefski et al., 2001). Although acceptance, seems to be an adaptive process, its higher level can be an indicator of no control over the environment and an inability to influence events (Garnefski et al., 2001). Consistent with this finding, a study on ER and anxiety reported that further anxiety traits were positively correlated with more acceptance (Jacob \& Anto, 2016). Therefore, acceptance could sometimes refer to the negative form of regulating emotions and adapting to a situation (Garnefski et al., 2001).

The current study highlighted the effect of the ER strategies in IAD by investigating the relationship between ER and interpretation bias. Lower levels of reappraisal and planning along with higher levels of rumination and catastrophizing were correlated with higher interpretation bias for health-related data. This finding is in line with other studies on the impact of interpretation bias in IAD (Warwick \& Salkovskis, 1990; Marcus, Hughes \& Arnau, 2008). Constant thinking about catastrophic consequences of health-related information (i.e. rumination) might be related to individuals' inability to foster alternative appraisals for a situation and selecting the most positive and appropriate one (Akbari, Dehghani, Khatibi, \& Vervoort, 2016). Moreover, such ruminative catastrophic appraisals might be implicated by some individuals; they believe thinking about worse conditions might increase their ability in detecting a threat (e.g. bodily sensations) and coping with that (e.g. the treatment of a disease). The observed positive correlations between interpretation bias, catastrophizing, and rumination, as well as their negative association with reappraisal, could be considered as supporting evidence. This notion can also be endorsed by our findings concerning a negative association between interpretation bias and refocus on planning. Refocus on planning is a functional problemsolving strategy; it provides individuals an opportunity to apply appropriate actions to solve the problem, rather than repetitively thinking about negative consequences (Garnefski et al., 2001). The more repetitive thinking about misinterpretations, the less application of practical plans. On the other hand, the relationship between interpretation bias and suppression was not significant. It might be because suppression is not necessarily a maladaptive strategy regarding its context (Bardeen and Fergus, 2014). Therefore, individuals with higher and lower levels of interpretation bias might apply suppression equally; making this strategy independent of interpretation bias. This finding is consistent with those of Gillian et al., (2010), who argued high catastrophizers did not necessarily apply suppression to regulate their emotions.

This study had some limitations that should be considered when interpreting the findings. The samples in the IAD and control groups were university students; it restricts generalizing the results to other populations. Besides, the research samples were recruited through a volunteer catchment rather than those seeking help in clinics. Therefore, the level of dysfunctionality in our sample might be less than those who felt the need of visiting a psychologist. This may increase the odds of typeII error in our analyses, which has to be addressed when interpreting the results.

Despite the mentioned limitations, the present research was one of the few studies that compared ER strategies between IAD and control groups. Furthermore, it went beyond evaluating two main strategies of reappraisals and suppression. Based on our results, the less application of reappraisal and refocus on planning, as well as more usage of rumination and catastrophizing, could be crucial maintenance factors to prone individuals to more severe IAD cases. We also found how considering interpretation bias for health-related data may highlight the impact of ER in IAD. Interpretation bias could make individuals ruminatively think about the catastrophic consequences of bodily changes that interrupt more positive reappraisal or practical problem-solving strategies. This might increase the odds of experiencing more severe IAD types. However, further studies are required to explore the relationship between ER strategies and other components of IAD, like avoidance and sensitivity to bodily symptoms.

\section{Conclusion}

Both functional (e.g. reappraisal \& planning) and dysfunctional strategies (e.g. rumination \& catastrophizing) contributed to the psychopathology of IAD. The biased interpretation of bodily information could make individuals prone to ruminate about the catastrophic consequences of bodily changes; such conditions interrupt 
fostering more positive reappraisal or practical problemsolving strategies.

\section{Ethical Considerations}

\section{Compliance with ethical guidelines}

All ethical principles were considered in this article. The participants were informed about the purpose of the research and its implementation stages; they were also assured about the confidentiality of their information; Moreover, They were allowed to leave the study whenever they wish, and if desired, the results of the research would be available to them.

\section{Funding}

This research did not receive any specific grant from funding agencies in the public, commercial, or not-forprofit sectors.

\section{Authors' contributions}

All authors contributed equally in preparing all parts of the research.

\section{Conflict of interest}

The authors declared no conflicts of interest.

Acknowledgments

We would like to express our thanks to all participants who attended our study.

\section{References}

Abramowitz, J. S., Brigidi, B. D., \& Foa, E. B. (1999). Health concerns in patients with obsessive-compulsive disorder. Journal of Anxiety Disorders, 13(5), 529-39. [DOI:10.1016/S08876185(99)00022-5]

Akbari, F., Dehghani, M., Khatibi, A., \& Vervoort, T. (2016). Incorporating family function into chronic pain disability: the role of catastrophizing. Pain Research and Management, 2016, 6838596. [DOI:10.1155/2016/6838596] [PMID] [PMCID]

American Psychiatric Association. (2013). Diagnostic and Statistical Manual of mental disorders (DSM-5®). New York: American Psychiatric Association Publishing. [DOI:10.1176/appi books.9780890425596
Bardeen, J. R., \& Fergus, T. A. (2014). An examination of the incremental contribution of emotion regulation difficulties to health anxiety beyond specific emotion regulation strategies. Journal of Anxiety Disorders, 28(4), 394-401. [DOI:10.1016/j. janxdis.2014.03.002] [PMID]

Barsky, A. J., Ettner, S. L., Horsky, J., \& Bates, D. W. (2001) Resource utilization of patients with hypochondriacal health anxiety and somatization. Medical Care, 39(7), 705-15 [DOI:10.1097/00005650-200107000-00007] [PMID]

Campbell-Sills, L., Ellard, K., \& Barlow, D. (2013). Emotion regulation in anxiety disorders. In J. Gross, Handbook of Emotion Regulation. New York: Guilford Press. [DOI:10.1016/j. janxdis.2013.06.007] [PMID]

Cioffi, D., \& Holloway, J. (1993). Delayed costs of suppressed pain. Journal of Personality and Social Psychology, 64(2), 274-82. [DOI:10.1037/0022-3514.64.2.274] [PMID]

Deacon, B., \& Abramowitz, J. S. (2008). Is hypochondriasis related to obsessive-compulsive disorder, panic disorder, or both? An empirical evaluation. Journal of Cognitive Psychotherapy, 22(2), 115. [DOI:10.1891/0889-8391.22.2.115]

Fergus, T. A., \& Valentiner, D. P. (2010). Disease phobia and disease conviction are separate dimensions underlying hypochondriasis. Journal of Behavior Therapy and Experimental Psychiatry, 41(4), 438-44. [DOI:10.1016/j.jbtep.2010.05.002] [PMID]

First, M. B., Williams, J. B. W., Karg, R. S., \& Spitzer, R. L. (2015). Structured clinical interview for DSM-5 disorders, clinician version (SCID-5-CV). Arlington, VA: American Psychiatric Association. https:// books.google.com/books?id=TqftrQEACAAJ\&dq

Garnefski, N., Kraaij, V., \& Spinhoven, P. (2001). Negative life events, cognitive emotion regulation and emotional problems. Personality and Individual Differences, 30(8), 1311-27. [DOI:10.1016/S0191-8869(00)00113-6]

Gerolimatos, L. A., \& Edelstein, B. A. (2012). Predictors of health anxiety among older and young adults. International Psychogeriatrics, 24(12), 1998-2008. [DOI:10.1017/S1041610212001329] [PMID]

Gilliam, W., Burns, J. W., Quartana, P., Matsuura, J., Nappi, C., \& Wolff, B. (2010). Interactive effects of catastrophizing and suppression on responses to acute pain: A test of an appraisal $\times$ emotion regulation model. Journal of Behavioral Medicine 33(3), 191-9. [DOI:10.1007/s10865-009-9245-0] [PMID] [PMCID]

Görgen, S. M., Hiller, W., \& Witthöft, M. (2014). Health anxiety, cognitive coping, and emotion regulation: A latent variable approach. International Journal of Behavioral Medicine, 21(2), 364-74. [DOI:10.1007/s12529-013-9297-y] [PMID]

Gross, J. J. (1998). The emerging field of emotion regulation: An integrative review. Review of General Psychology, 2(3), 271 [DOI:10.1037/1089-2680.2.3.271]

Gross, J. J., \& John, O. P. (2003). Individual differences in two emotion regulation processes: Implications for affect, relationships, and well-being. Journal of Personality and Social Psychology, 85(2), 348. [DOI:10.1037/0022-3514.85.2.348] [PMID]

Gross, J. J., \& Levenson, R. W. (1993). Emotional suppression: Physiology, self-report, and expressive behavior. Journal of Personality and Social Psychology, 64(6), 970-86. [DOI:10.1037/00223514.64.6.970] [PMID] 
Haenen, M. A., de Jong, P. J., Schmidt, A. J., Stevens, S., \& Visser, L. (2000). Hypochondriacs' estimation of negative outcomes: domain-specificity and responsiveness to reassuring and alarming information. Behaviour Research and Therapy, 38(8), 819-33. [DOI:10.1016/S0005-7967(99)00128-X]

Jacob, S., \& Anto, M. M. (2016). A study on cognitive emotion regulation and anxiety and depression in adults. The International Journal of Indian Psychology, 3(2), 118-24. http:/ / oaji.net/ articles/2016/1170-1452464986.pdf

Koster, E. H., Rassin, E., Crombez, G., \& Näring, G. W. (2003). The paradoxical effects of suppressing anxious thoughts during imminent threat. Behaviour Research and Therapy, 41(9), 1113-20. [DOI:10.1016/S0005-7967(03)00144-X]

Kulpa, M., Ziętalewicz, U., Kosowicz, M., Stypuła-Ciuba, B., \& Ziółkowska, P. (2016). Anxiety and depression and cognitive coping strategies and health locus of control in patients with ovary and uterus cancer during anticancer therapy. Contemporary Oncology, 20(2), 171-5. [DOI:10.5114/wo.2016.60074] [PMID] [PMCID]

Lyubomirsky, S., Tucker, K. L., Caldwell, N. D., \& Berg, K. (1999). Why ruminators are poor problem solvers: Clues from the phenomenology of dysphoric rumination. Journal of Personality and Social Psychology, 77(5), 1041-60. [DOI:10.1037/00223514.77.5.1041] [PMID]

Marcus, D. K., Gurley, J. R., Marchi, M. M., \& Bauer, C. (2007). Cognitive and perceptual variables in hypochondriasis and health anxiety: A systematic review. Clinical Psychology Review, 27(2), 127-39. [DOI:10.1016/j.cpr.2006.09.003] [PMID]

Marcus, D. K., Hughes, K. T., \& Arnau, R. C. (2008). Health anxiety, rumination, and negative affect: A mediational analysis. Journal of Psychosomatic Research, 64(5), 495-501. [DOI:10.1016/j. jpsychores.2008.02.004] [PMID]

Pilowsky, I. (1967). Dimensions of hypochondriasis. The British Journal of Psychiatry, 113(494), 89-93. [DOI:10.1192/ bjp.113.494.89] [PMID]

Rief, W., Hiller, W., \& Margraf, J. (1998). Cognitive aspects of hypochondriasis and the somatization syndrome. Journal of Abnormal Psychology, 107(4), 587-95. [DOI:10.1037/0021843X.107.4.587] [PMID]

Salkovskis, P. M., Rimes, K. A., Warwick, H. M. C., \& Clark, D. M. (2002). The Health Anxiety Inventory: Development and validation of scales for the measurement of health anxiety and hypochondriasis. Psychological Medicine, 32(5), 843-53. [DOI:10.1017/S0033291702005822] [PMID]

Warwick, H. M., \& Salkovskis, P. M. (1990). Hypochondriasis. Behaviour Research and Therapy, 28(2), 105-17. [DOI:10.1016/00057967(90)90023-C]

Weck, F., Neng, J. M., Richtberg, S., \& Stangier, U. (2012). Dysfunctional beliefs about symptoms and illness in patients with hypochondriasis. Psychosomatics, 53(2), 148-54. [DOI:10.1016/j. psym.2011.11.007] [PMID]

Wegner, D. M., Schneider, D. J., Carter, S. R., \& White, T. L. (1987). Paradoxical effects of thought suppression. Journal of Personality and Social Psychology, 53(1), 5. [DOI:10.1037/00223514.53.1.5] 


\title{
Research Paper: Comparing Copper Serum Level and Cognitive Functioning in Patients With Schizophrenia and Healthy Controls
}

\author{
Shahrzad Mazhari' ${ }^{1}$, Shokouh Arjmand ${ }^{1}$, , Mahin Eslami Shahrbabaki², Elham Karimi Ghoughari ${ }^{* *}$ (i)
}

1. Neuroscience Research Centre, In stitute of Neuropharmacology, Kerman University of Medical Sciences, Kerman, Iran.

2. Department of Psychiatry, School of Medicine, Kerman University of Medical Sciences, Kerman, Iran.

Crtation: Mazhari, Sh., Arjmand, Sh., Eslami Shahrbabaki, M., \& Karimi Ghoughari, E. (2020). Comparing Copper Serum Level and Cognitive Functioning in Patients With Schizophrenia and Healthy Controls. Basic and Clinical Neuroscience, 11(5), 649-658. http://dx.doi.org/10.32598/bcn.9.10.11.5.2116.1

http://dx.doi.org/10.32598/bcn.9.10.11.5.2116.1

Article info:

Received: 07 Oct 2019

First Revision: 20 Oct 2019

Accepted: 13 Apr 2020

Available Online: 01 Sep 2020

Keywords:

Cognition, Copper,

Magnesium, Schizophrenia,

Trace elements, Brief

Assessment of Cognition in

Schizophrenia (BACS)

\begin{abstract}
A B S T RA C T
Introduction: The altered serum profiles of several trace elements have been reported in Schizophrenia (SCZ). This study was designed to elucidate whether the serum levels of Copper $(\mathrm{Cu})$ and Magnesium $(\mathrm{Mg})$, the two essential trace elements which contribute to neurotransmitter transmission, are altered in patients with SCZ. We also investigated whether there is an interrelation between cognitive functioning and the serum levels of $\mathrm{Cu}$ and $\mathrm{Mg}$.

Methods: Sixty patients with SCZ and 30 healthy controls participated in this study. The patient group was divided into the following: i) early patients $(n=35, \leq 5$ years of illness initiation), and ii) chronic patients ( $\mathrm{n}=25, \geq 5$ years of illness duration). The serum levels of $\mathrm{Cu}$ and $\mathrm{Mg}$ were measured by atomic absorption spectroscopy and ion-selective electrode potentiometry, respectively. To assess cognitive abilities, a Persian adaptation of the Brief Assessment of Cognition in Schizophrenia (BACS) was administered.

Results: The present research results revealed significantly higher $\mathrm{Cu}$ serum levels in both patient groups [early patients ( $M=94.6)$, chronic patients $(M=97.5)$ ], compared to the controls $(\mathrm{M}=71.0)(\mathrm{P}<0.001)$; however, no significant difference was observed among the study groups for $\mathrm{Mg}$ [patients with the recent onset $(\mathrm{M}=2.0)$, chronic patients $(\mathrm{M}=2.0)$, and controls $(\mathrm{M}=1.9)]$ $\mathrm{P}=0.1$. While the serum $\mathrm{Cu}$ profile of healthy individuals revealed a negative correlation with working memory $(\mathrm{r}=-0.42, \mathrm{P}=0.02)$, and executive functioning $(\mathrm{r}=-0.40, \mathrm{P}=0.03)$, no significant correlation was observed between $\mathrm{Cu}$ serum levels of patients and BACS cognitive domains.

Conclusion: findings suggested that the high $\mathrm{Cu}$ serum concentration might impact the cognitive decline in healthy individuals; however, no significant correlation was observed in the Patients; i.e. most likely because cognition is severely impaired in SCZ. Additional studies examining trace elements in drug-naïve patients with $\mathrm{SCZ}$ are required.
\end{abstract}




\section{Highlights}

- Schizophrenia (SCZ) is a severe debilitating disorder that affects emotion, thought, etc.

- Recently, the cognitive deficits have been considered as a core feature of SCZ.

- The main assumption in explaining the pathophysiology of SCZ is dysfunctional dopaminergic, glutamatergic, and GABAergic transduction

\section{Plain Language Summary}

Schizophrenia (SCZ) is a severe disorder that affects emotion, thought, cognition, and behavior. Within the last few years, cognitive deficits have been considered as a core feature of SCZ. Furthermore, numerous studies have focused on understanding the basis of cognitive decline to better manage the disorder and improve patients' quality of life. The main assumption in explaining the pathophysiology of SCZ is dysfunctional dopaminergic, glutamatergic, and GABAergic transduction. This study aimed to investigate the possible correlation between cognitive functioning in patients with SCZ and their $\mathrm{Cu}$ and $\mathrm{Mg}$ serum levels.

\section{Introduction}

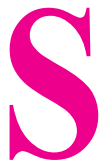

chizophrenia (SCZ) is a severe debilitating neurodevelopmental disorder that affects emotion, thought, cognition, and behavior (Rahman et al., 2009). It affects roughly $1 \%$ of the population globally. Besides, it is recognized by heterogeneous symptoms, ranging from negative symptoms; psychotic symptoms; and disorganized behavior/speech, to the loss of goal-directed activities and impaired socioeducational functioning (Cai et al., 2015; Saha, Chant, Welham, \& McGrath, 2005). Despite extensive research, the exact pathophysiology of SCZ remains unknown and not well-established; we only possess a sketchy picture displaying miscellaneous and disparate contributing factors, varying from genetic and metabolic to environmental ones (van Os \& Kapur, 2009).

Within the last few years, cognitive deficits have been considered as a core feature of SCZ. Furthermore, numerous studies have focused on understanding the basis of cognitive decline to better manage the disorder and improve patients' quality of life (Green \& Harvey, 2014; Tripathi, Kar, \& Shukla, 2018).

The altered amounts of trace elements have been linked to reduced cognitive functions in the elderly (Lam et al., 2008; Smorgon et al., 2004). Furthermore, previous studies reported that the serum or tissue levels of several trace elements are altered in patients with SCZ; they developed the trace element hypothesis of SCZ (Ghanem et al., 2009; Rahman et al., 2009; Yanik, Kocyigit,
Tutkun, Vural, \& Herken, 2004). Such findings were followed by a great body of interventions to search for possible biological markers representing the altered levels of trace elements in SCZ (Cai et al., 2015; Fryar-Williams \& Strobel, 2015; Lin et al., 2017). However, the related data are controversial and there exist some ongoing debates about the contributing role of some trace elements in SCZ (Cai et al., 2015; Devanarayanan, Nandeesha, Kattimani, Sarkar, \& Jose, 2016; Ordak, Matras, Muszynska, Nasierowski, \& Bujalska-Zadrozny, 2017).

The main assumption in explaining the pathophysiology of SCZ is dysfunctional dopaminergic, glutamatergic, and GABAergic transduction (de Jonge, Vinkers, Hulshoff Pol, \& Marsman, 2017; Howes, McCutcheon, \& Stone, 2015; Javitt, 2010). Moreover, trace elements are responsible for numerous intricate biological processes and of great significance for the efficacious functioning of the cells (Fraga, 2005; Osredkar \& Sustar, 2011). Copper $(\mathrm{Cu})$ is a bivalent cation and an essential ligand for some metalloenzymes, including Dopamine $\beta$-Hydroxylase (DBH) (Vendelboe et al., 2016), i.e. a $\mathrm{Cu}$-dependent oxygenase, a key component of catecholamine biosynthesis; and has been demonstrated to be impaired in SCZ (Cubells \& Zabetian, 2004; Cubells et al., 2011; Osredkar \& Sustar, 2011; Tang, Yao, Li, Lin, \& Huang, 2018). Another bivalent cation, Magnesium $(\mathrm{Mg})$, has been proven to impact GABAergic and glutamatergic transmission; both have been implicated dysfunctional in SCZ (Ordak et al., 2017).

Thus, this study aimed to investigate the possible correlation between cognitive functioning in patients with 
Table 1. The demographic and clinical characteristics of the study participants

\begin{tabular}{|c|c|c|c|c|}
\hline \multirow{2}{*}{ Variabels } & \multicolumn{3}{|c|}{ Mean $\pm S D$} & \multirow{2}{*}{$\mathbf{P}$} \\
\hline & Controls (C) $n=30$ & Early patients (EP) $n=35$ & Chronic patients $(C P) n=25$ & \\
\hline Age (years) & $33.5 \pm 7.8$ & $31.3 \pm 8.7$ & $37.3 \pm 7.8$ & $0.03 ; C P>E P>C$ \\
\hline Education (years) & $6.1 \pm 1.1$ & $5.3 \pm 1.8$ & $5.1 \pm 1.8$ & $0.04 ; C>E P=C P$ \\
\hline Sex (males) ${ }^{\mathrm{a}}$ No.(\%) & $24(80 \%)$ & $29(83 \%)$ & $19(76 \%)$ & NS \\
\hline $\mathrm{Cu}$ & $71.0 \pm 10.8$ & $94.6 \pm 27.2$ & $97.5 \pm 26.5$ & $<0.001 ; \mathrm{EP}=\mathrm{CP}>\mathrm{C}$ \\
\hline $\mathrm{Mg}$ & $1.9 \pm 0.14$ & $2.0 \pm 0.16$ & $2.0 \pm 0.15$ & NS \\
\hline $\begin{array}{l}\text { of the duration of illness } \\
\text { (years) }\end{array}$ & - & $2.56 \pm 1.6$ & $13.1 \pm 5.5$ & $<0.001$ \\
\hline PANSS-Positive & - & $14.4 \pm 3.8$ & $14.8 \pm 3.6$ & NS \\
\hline PANSS-Negative & - & $14.4 \pm 2.7$ & $14.7 \pm 4.1$ & NS \\
\hline PANSS-other & - & $28.9 \pm 6.8$ & $29.2 \pm 7.8$ & NS \\
\hline $\begin{array}{l}\text { Mean chlorpromazine } \\
\text { equivalent (mg) }\end{array}$ & - & $822.1 \pm 510.4$ & $1002.1 \pm 472.2$ & NS \\
\hline
\end{tabular}

PANSS: Positive and Negative Symptom Scale; ${ }^{a}: \chi^{2}$-test.

NEUR SCIENCE

SCZ and their $\mathrm{Cu}$ and $\mathrm{Mg}$ serum levels. Previous study findings on the serum levels of $\mathrm{Cu}$ and $\mathrm{Mg}$ in patients with SCZ have been inconsistent and controversial (Cai et al., 2015; Devanarayanan et al., 2016; Ordak et al., 2017). Besides, to our knowledge, there are few, if any study to examine the association between the serum levels of these trace elements and cognitive functioning in SCZ.

Accordingly, the serum levels of $\mathrm{Mg}$ and $\mathrm{Cu}$ in patients with SCZ were measured. Then, a probable existing correlation between the serum profile of $\mathrm{Cu}$ and $\mathrm{Mg}$ and the cognitive functioning of patients with SCZ, as well as healthy participants were explored. For this purpose, a reliable and valid instrument, namely the Brief Assessment of Cognition in Schizophrenia (BACS), was used.

\section{Materials and Methods}

A group of 60 patients (48 males) was recruited through consecutive admissions to a psychiatric hospital in Kerman, Iran. The study patients were divided into two groups based on the chronicity of their illness: i) early patients $(n=35, \leq 5$ years of illness initiation), and ii) chronic patients ( $n=25,>5$ years of illness duration). Clinical symptoms were assessed by the Positive and Negative Syndrome Scale (PANSS) (Kay, Opler, \& Lindenmayer, 1988). The study patients were all receiving antipsychotic medications and clinically stable at the time of the experiment. The Mean $\pm \mathrm{SD}$ chlorpromazine equivalent was 897.2 (498.8) mg (Woods, 2003).
The control group consisted of 30 healthy participants (24 males) without a personal or familial history of psychotic disorders. Head injury, neurological disorders, and substance abuse at the time of the experiment were the exclusion criteria for all study participants. The study was conducted under the principles of the Declaration of Helsinki for Biomedical Research. A written informed consent form was obtained from all research participants. The study was approved by the Ethics Committees of Kerman University of Medical Sciences (code: EC/95-38/KNRC).

Fasting blood samples $(5 \mathrm{~mL})$ were collected from the antecubital vein of all study participants; we used a plastic syringe with a stainless-steel needle, between 7.00 AM to 9.00 AM. Each blood sample was poured into a metalfree plastic tube and clotted at room temperature for 30 minutes. Then, the serum was separated by centrifuging it at $3000 \mathrm{rpm}$ for 15 minutes and preserved at $-20^{\circ} \mathrm{C}$ until assayed. The concentrations of $\mathrm{Cu}$ and $\mathrm{Mg}$ were measured by atomic absorption spectrophotometer (PG instrument, AA500), and ion-selective electrode potentiometry (COBAS INTEGRA ${ }^{\circledR} 400$ plus), respectively. $\mathrm{Mg}$ and $\mathrm{Cu}$ values were expressed in milligrams per deciliter $(\mathrm{mg} / \mathrm{dL})$ and micrograms per deciliter (ug/dL), respectively.

A Persian adaptation of the BACS was administered to assess cognitive functioning (Mazhari et al., 2014). The BACS, a performance-based cognitive assessment battery, is specifically designed to examine the cognitive 
Table 2. The mean and standard deviation scores of the cognitive measures of the study participants

\begin{tabular}{|c|c|c|c|c|}
\hline \multirow{2}{*}{ Variabels } & \multicolumn{3}{|c|}{ Mean $\pm S D$} & \multirow{2}{*}{$\mathbf{P}$} \\
\hline & Controls $n=30$ & Early Patients (EP) n=35 & Chronic Patients (CP) n=25 & \\
\hline BACS z-score & $-0.08 \pm 0.6$ & $-0.9 \pm 0.6$ & $-1.1 \pm 0.6$ & $<0.001 ; \mathrm{C}<\mathrm{EP}=\mathrm{CP}$ \\
\hline Verbal memory & $36.3 \pm 10.4$ & $26.7 \pm 11.6$ & $21.4 \pm 9.3$ & $<0.001 ; \mathrm{C}>\mathrm{EP}=\mathrm{CP}$ \\
\hline Working memory & $15.1 \pm 5.1$ & $11.8 \pm 4.5$ & $10.8 \pm 5.1$ & $0.005 ; C>E P=C P$ \\
\hline Motor speed & $66.5 \pm 15.3$ & $47.6 \pm 15.7$ & $39.8 \pm 17.1$ & $<0.001 ; \mathrm{C}>\mathrm{EP}=\mathrm{CP}$ \\
\hline Verbal fluency & $19.9 \pm 4.7$ & $14.7 \pm 7.5$ & $13.7 \pm 9.2$ & $0.02 ; \mathrm{C}>\mathrm{CP}, \mathrm{EP}=\mathrm{CP}$ \\
\hline $\begin{array}{l}\text { Attention \& speed of } \\
\text { information processing }\end{array}$ & $34.5 \pm 10.3$ & $20.1 \pm 10.9$ & $17.1 \pm 7.6$ & $<0.001 ; \mathrm{C}>\mathrm{EP}=\mathrm{CP}$ \\
\hline Executive function & $15.4 \pm 5.7$ & $12.3 \pm 7.4$ & $11.6 \pm 6.1$ & $<0.001 ; C>E P=C P$ \\
\hline
\end{tabular}

NEURSIENCE

function of SCZ, requiring 2.5 hours of testing (Keefe et al., 2004). The BACS is sensitive to cognitive impairment in SCZ, has high test-retest reliability, and is related to functional outcome (Keefe et al., 2004). The BACS assesses the 6 cognitive domains found to be consistently impaired, including verbal memory, verbal fluency, working memory, motor speed, attention, and executive function. The standardized z-scores from each test were summed; the composite score is the z-score of that sum. A lower score reflects greater cognitive impairment.

The obtained demographic data were compared between the groups using a combination of Chi-squared test and one-way Analysis of Variance (ANOVA). Spearman's correlation coefficient was used to examine the correlation between $\mathrm{Cu}$ and $\mathrm{Mg}$ serum levels, and cognitive functioning. Backward multiple linear regression analysis was performed to explore the relationship between the BACS composite z-scores (dependent variable) and the independent variables, including the serum levels of $\mathrm{Cu}$, the years of education, and the group. The variable of the group was coded as 0 (control group), 1 (early patients), and 2 (chronic patients). It was also offered as an ordinal variable to the multifactorial model.

\section{Results}

Table 1 lists the demographic and clinical characteristics of the study participants. The obtained results suggested differences in the mean age of the control and the patient groups; the mean age of the chronic patients was higher than that of the control and the early patient groups. Moreover, both patient groups had significantly fewer years of education than the controls. Both patient groups and the control group were well-matched for gender $(\mathrm{P}>0.05)$. The serum levels of $\mathrm{Cu}$ were significantly different between the three groups. Besides, both patient groups presented higher serum levels than that of the controls; the difference between the two patient groups was not significant. However, the serum levels of $\mathrm{Mg}$ were not significantly different between the control and both patients groups.

In all cognitive domains of the BACS, both patient groups achieved significantly lower values than the healthy controls. Moreover, chronic patients obtained the minimum scores in all cognitive domains (Table 2).

To examine the effects of $\mathrm{Cu}$ and $\mathrm{Mg}$ serum levels on cognitive functions, the correlation coefficients were calculated between $\mathrm{Cu}$ and $\mathrm{Mg}$ levels and the BACS cognitive domains for the control and patient groups, separately. For $\mathrm{Cu}$, the relevant results indicated that $\mathrm{Cu}$ levels had significant negative correlations with working memory $(\mathrm{r}=0.42, \mathrm{P}=0.02)$, and executive function $(\mathrm{r}=0.40, \mathrm{P}=0.03)$ in the control group. However, there were no significant correlations between Culevel and cognitive functioning in the patient groups. For $\mathrm{Mg}$, the related data signified no significant correlations between $\mathrm{Mg}$ level and cognitive functions in any of the study groups ( $\mathrm{P}>0.05$ for all variables).

Next, we explored whether $\mathrm{Cu}$ serum levels could predict the cognitive performances of the study participants. The controls had significantly more years of education than the patients; thus, education was also considered as an independent variable. The collected results suggested an overall fit of the model $(\mathrm{df}=4, \mathrm{~F}=25.7, \mathrm{P}<0.001)$. All variables were significant predictors of the BACS composite $\mathrm{z}$-score $(\mathrm{P}<0.05)$. As per Table 3, increased 
Table 3. The interrelationship of the BACS composite z-score with serum copper level, education, and group using multiple regression analysis

\begin{tabular}{cccc}
\hline Variabels & B & Beta & P \\
\hline Serum Cu level & -0.02 & -0.53 & 0.003 \\
\hline Education & 0.21 & 0.41 & $<0.001$ \\
\hline Group (controls=0) & -1.23 & -1.25 & $<0.001$ \\
\hline Group*Cu & 0.01 & 1.14 & 0.006 \\
\hline
\end{tabular}

NEUR SCIENCE

educational level was associated with enhanced BACS composite z-score (i.e. the coefficient of the education represents the mean change of the BACS composite zscore). Additionally, the average difference in BACS between the control and patient groups was -1.23 . Moreover, lower years of education, increased $\mathrm{Cu}$ serum level, and being diagnosed with SCZ were accompanied by reduced BACS composite z-scores. The interaction between the group type and $\mathrm{Cu}$ level was also significant $(\mathrm{P}=0.006)$; accordingly, the high $\mathrm{Cu}$ serum levels affected cognition in the control group and it is distinct from the patients (i.e. the coefficient of group ${ }^{*} \mathrm{Cu}$ means that the effect of $\mathrm{Cu}$ on BACS was different for the control and patient groups). This finding highlights that in the control group, increased $\mathrm{Cu}$ serum levels could predict faulty performances in the BACS cognitive domains, but not in patients with SCZ. Figure 1 provides a scatter plot of the correlation between $\mathrm{Cu}$ serum levels and the BACS composite z-scores. This plot demonstrates poor correlations between $\mathrm{Cu}$ and BACS composite z-score for both patient groups; however, it was nearly strong for the controls. Given the negative slope in Figure 1, the BACS composite z-score decreased, as $\mathrm{Cu}$ increased.

Finally, to examine the effect of clinical characteristics of patients on the $\mathrm{Cu}$ serum level in SCZ, the correlation coefficients (Spearman $r$ ) were evaluated. There was no significant correlation between serum $\mathrm{Cu}$ levels and PANSS positive, and negative symptoms ( $\mathrm{P}>0.2)$. Furthermore, no correlation was observed between $\mathrm{Cu}$ serum levels and chlorpromazine equivalent dose $(\mathrm{P}=0.2)$.

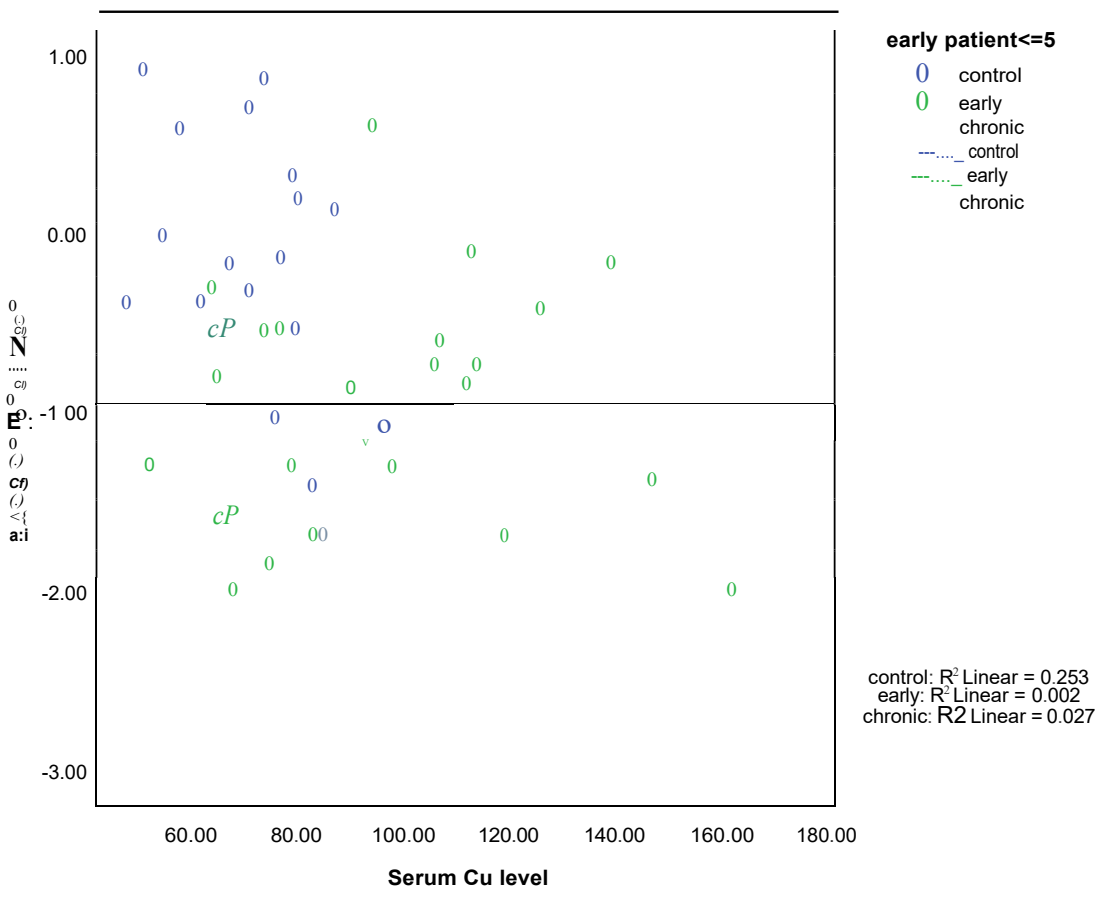

NEUR SCIENCE

Figure 1. The scatter plot of the BACS composite z-score and serum Cu level in the groups of patients (early, chronic), and healthy controls 


\section{Discussion}

To the best of our knowledge, the present study was the first attempt to understand whether there is an association between the serum levels of two important trace elements whose functions seem to contribute to the pathophysiology of SCZ and their cognitive functioning.

We found significantly high levels of $\mathrm{Cu}$, but not $\mathrm{Mg}$, in the explored patients with SCZ, compared to the healthy control group. Numerous controversial reports are comparing the tissue and serum levels of several trace elements, including $\mathrm{Cu}$ and $\mathrm{Mg}$ in patients with SCZ and healthy individuals (Cai et al., 2015; Devanarayanan et al., 2016; Ordak et al., 2017).

The selected patients who met the present research inclusion criteria were all on antipsychotics. Our results were in-line with those of several studies on $\mathrm{Cu}$ serum levels in patients with SCZ (Devanarayanan et al., 2016; Ghanem et al., 2009; Rahman et al., 2009; Vidović et al., 2013; Yanik et al., 2004). Several lines of evidence have concluded that the elevation of plasma/serum $\mathrm{Cu}$ concentration may be due to treatment with various antipsychotics. A study has reported higher levels of $\mathrm{Cu}$ in patients receiving depot neuroleptics relative to those who were not on antipsychotics. However, a study reported increased $\mathrm{Cu}$ serum levels even in medication-free patients with SCZ (Devanarayanan et al., 2016). Contrarily, there is a report indicating lower levels of $\mathrm{Cu}$ in patients with $\mathrm{SCZ}$ (Liu et al., 2015); however, most findings have focused on the elevated levels of $\mathrm{Cu}$. Further studies and meta-analyses are required to draw a more precise conclusion on the serum levels of $\mathrm{Cu}$ in patients with SCZ. Additionally, possible mechanisms by which antipsychotics might lead to excessive $\mathrm{Cu}$ serum levels should be further investigated.

Furthermore, no significant correlation was detected between the serum level of $\mathrm{Cu}$ and the cognitive functioning in patients with SCZ. In agreement with previous findings, $\mathrm{Cu}$ serum levels in healthy individuals were negatively correlated with their cognitive performance (composite z-score). The higher the $\mathrm{Cu}$ level, the worse cognitive performance in healthy participants. With a more meticulous look into each individual component of the BACS, $\mathrm{Cu}$ serum levels in the healthy control group exhibited a negative correlation; specifically, with the working memory and executive functioning.

It is suggested that an increased concentration of $\mathrm{Cu}$ could lead to cognitive decline in healthy individuals, owing to free $\mathrm{Cu}$ deregulation (Klevay, 2010). Additionally, Zhou et al. have reported decreased working memory and cognition in children, especially males; as the increased $\mathrm{Cu}$ serum level, exceeded the required amount for metabolic processes (Zhou et al., 2015). Another study has also stated that elevated $\mathrm{Cu}$ plasma level is linked to reduced cognitive functions in the elderly. Besides, they have discussed that the aggregation of amyloid beta-protein due to interactions with $\mathrm{Cu}$ as a reason for such findings (Gao et al., 2008).

In this regard, Salustri et al. have presented the interrelation between higher free $\mathrm{Cu}$ serum levels and reduced cognitive functions in healthy populations. They also argued that free $\mathrm{Cu}$ may act by disrupting the neurons of locus coeruleus (Salustri et al., 2010), the foremost site for the synthesis of the brain's norepinephrine (Sara, 2009). On the other hand, studies exhibited the principal involvement of the locus coeruleus and noradrenergic system in cognitive processes and working memory (Berridge \& Waterhouse, 2003; Borodovitsyna, Flamini, \& Chandler, 2017; Sara, 2009).

Contrary to the healthy controls, no significant correlation was detected between the serum level of $\mathrm{Cu}$ and their cognitive functioning in patients with SCZ. Decreased DBH activity and noradrenergic transmission in patients with $\mathrm{SCZ}$ have been established a blunted catecholamine synthesis (Cubells \& Zabetian, 2004; Rahman, Rahman, Rahman, \& Kato, 2009). On the other hand, ionized $\mathrm{Cu}$ actively contributes as a ligand for the enzymatic activity of DBH. Moreover, an excessive amount of $\mathrm{Cu}$ may be engaged in the structure of DBH; accordingly, it boosts the conversion of dopamine into norepinephrine, with the final repercussion of decreased dopaminergic activity and increased synthesis of epinephrine from the noradrenergic neurons of the locus coeruleus. Therefore, we presumed that it might be an explanation to understand why excessive $\mathrm{Cu}$ is not attributed to declined cognitive functioning in patients with SCZ, unlike the healthy participants. It might be also induced by the pre-existing dysfunctional activity of locus coeruleus' noradrenergic neurons in patients with $\mathrm{SCZ}$ despite the Cu-dependent disturbance of locus coeruleus neurons in healthy individuals. Another probable reason might be that in patients with SCZ, cognition is markedly impaired and $\mathrm{Cu}$ serum concentrations can hardly leave a noticeable impact on it; therefore, no significant association was observed in this regard.

Per previous findings (Devanarayanan et al., 2016; Ghanem et al., 2009), we found no significant correlation between higher $\mathrm{Cu}$ serum level and PANSS score. Thus, increased $\mathrm{Cu}$ concentration does not reflect the severity of SCZ.

Eventually, we observed no significant alternation in the $\mathrm{Mg}$ serum concentration; it may be as a result of receiving antipsychotics. Our data are in alignment with a study conducted by

Nechifor, Vaideanu, Palamaru, Borza, and Mindreci, (2004), i.e. indicative of lower erythrocyte; but not com- 
paring $\mathrm{Mg}$ serum levels in healthy controls, which then got normalized after receiving treatment and during remission. A finding led to this deduction that increased intracellular $\mathrm{Mg}$ level is associated with enhanced GABAergic activity, and reduced glutamatergic and dopaminergic transmission (Ordak et al., 2017). In the face of these data, another contradictory finding has presented elevated platelet $\mathrm{Mg}$ concentration in suicidal patients with SCZ (Ruljancic, Mihanovic, Cepelak, \& Bakliza, 2013). Ordak et al. (2017) highlighted that such inconsistencies may be due to extracellular measurements of $\mathrm{Mg}$ instead of intracellular and ionized ones. This is because only around $1 \%$ of the total $\mathrm{Mg}$ is distributed in the plasma.

\section{Conclusion}

Our study had several limitations. First, free (ionized) $\mathrm{Cu}$ can actively cross the blood-brain barrier and has a more crucial role in cognition than that of the bound one; thus, future studies are recommended measuring the ionized and non-ionized $\mathrm{Cu}$ levels. Moreover, we recommend performing a study on medicated and non- medicated patients to better recognize whether elevated $\mathrm{Cu}$ serum levels are related to antipsychotic therapy or not.

\section{Ethical Considerations}

\section{Compliance with ethical guidelines}

All ethical principles are considered in this article. The participants were informed about the purpose of the research and its implementation stages; they were also assured about the confidentiality of their information; moreover, they were free to leave the study whenever they wished, and if desired, the research results would be available to them.

\section{Funding}

The current study was supported by the Neuroscience Research Center of Kerman University of Medical Sciences (Code: 95-38/KNRC).

\section{Authors' contributions}

Conceptualization, Investigation, Writing - review \& editing: Shahrzad Mazhari, Elham Karimi Ghoughari, and Mahin Eslami Shahrbabaki; Data Collection: Shokouh Arjmand; All authors have contributed significantly, and all are in agreement with the content of the manuscript.
The authors declared no conflicts of interest.

\section{References}

Berridge, C. W., \& Waterhouse, B. D. (2003). The locus coeruleus-noradrenergic system: Modulation of behavioral state and state-dependent cognitive processes. Brain Research Reviews, 42(1), 33-84. [DOI:10.1016/S0165-0173(03)00143-7]

Borodovitsyna, O., Flamini, M., \& Chandler, D. (2017). Noradrenergic Modulation of Cognition in Health and Disease. Neural Plasticity, 2017, 1-14. [DOI:10.1155/2017/6031478] [PMID] [PMCID]

Cai, L., Chen, T., Yang, J., Zhou, K., Yan, X., \& Chen, W., et al. (2015). Serum trace element differences between Schizophrenia patients and controls in the Han Chinese population. Scientific Reports, 5, 15013. [DOI:10.1038/srep15013] [PMID] [PMCID]

Cubells, J. F., \& Zabetian, C. P. (2004). Human genetics of plasma dopamine $\beta$-hydroxylase activity: Applications to research in psychiatry and neurology. Psychopharmacology, 174(4), 463-76. [DOI:10.1007/s00213-004-1840-8] [PMID]

Cubells, Joseph F, Sun, X., Li, W., Bonsall, R. W., McGrath, J. A., \& Avramopoulos, D., et al. (2011). Linkage analysis of plasma dopamine $\beta$-hydroxylase activity in families of patients with schizophrenia. Human Genetics, 130(5), 635-43. [DOI:10.1007/ s00439-011-0989-6] [PMID] [PMCID]

de Jonge, J. C., Vinkers, C. H., Hulshoff Pol, H. E., \& Marsman, A. (2017). GABAergic mechanisms in schizophrenia: linking postmortem and in vivo studies. Frontiers in Psychiatry, 8, 118. [DOI:10.3389/fpsyt.2017.00118] [PMID] [PMCID]

Devanarayanan, S., Nandeesha, H., Kattimani, S., Sarkar, S., \& Jose, J. (2016). Elevated copper, hs C-reactive protein and dyslipidemia in drug free schizophrenia: Relation with psychopathology score. Asian Journal of Psychiatry, 24, 99-102. [DOI:10.1016/J.AJP.2016.08.025] [PMID]

Fraga, C. G. (2005). Relevance, essentiality and toxicity of trace elements in human health. Molecular Aspects of Medicine, 26(4 5), 235-44. [DOI:10.1016/j.mam.2005.07.013] [PMID]

Fryar-Williams, S., \& Strobel, J. E. (2015). Biomarkers of a fivedomain translational substrate for schizophrenia and schizoaffective psychosis. Biomarker Research, 3(1), 3. [DOI:10.1186/ s40364-015-0028-1] [PMID] [PMCID]

Gao, S., Jin, Y., Unverzagt, F. W., Ma, F., Hall, K. S., \& Murrell, J. R., et al. (2008). Trace element levels and cognitive function in rural elderly Chinese. The Journals of Gerontology Series A: Biological Sciences and Medical Sciences, 63(6), 635-41. [DOI:10.1093/ gerona/63.6.635] [PMID] [PMCID]

Ghanem, A. A., Ali, E. M., El-Bakary, A. A., El-Morsy, D., \& Elkanishi, S. M., et al. (2009). Copper and Zinc levels in hair of both schizophrenic and depressed patients. Mansoura Journal of Forensic Medicine and Clinical Toxicology, 17(1), 89-102. [DOI:10.21608/ mifmct.2009.53299]

\section{Conflict of interest}


Green, M. F., \& Harvey, P. D. (2014). Cognition in schizophrenia: Past, present, and future. Schizophrenia Research: Cognition, 1(1), e1e9. [DOI:10.1016/j.scog.2014.02.001] [PMID] [PMCID]

Alvarez, C., \& Amado, J. A. (2000). Higher levels of serum copper in schizophrenic patients treated with depot neuroleptics. Psychiatry Research, 94(1), 51-8. [DOI:10.1016/S0165-1781(00)00126-8]

Howes, O., McCutcheon, R., \& Stone, J. (2015). Glutamate and dopamine in schizophrenia: An update for the 21st century. Journal of Psychopharmacology (Oxford, England), 29(2), 97- 115. [DOI:10.1177/0269881114563634] [PMID] [PMCID]

Javitt, D. C. (2010). Glutamatergic theories of schizophrenia. The Israel Journal of Psychiatry and Related Sciences, 47(1), 4-16. http:// sites.oxy.edu/clint/physio/article/Glutamatergictheoriesofschizophrenia.pdf

Kay, S. R., Opler, L. A., \& Lindenmayer, J. P. (1988). Reliability and validity of the positive and negative syndrome scale for schizophrenics. Psychiatry Research, 23(1), 99-110. [DOI:10.1016/01651781(88)90038-8]

Keefe, R., Goldberg, T. E., Harvey, P. D., Gold, J. M., Poe, M. P., \& Coughenour, L. (2004). The Brief Assessment of Cognition in Schizophrenia: Reliability, sensitivity, and comparison with a standard neurocognitive battery. Schizophrenia Research, 68(2-3), 283- 97. [DOI:10.1016/j.schres.2003.09.011] [PMID]

Klevay, L. M. (2010). Copper and cognition. Clinical Neurophysiology, 121(12), 2177. [DOI:10.1016/j.clinph.2010.04.025] [PMID]

Lam, P. K., Kritz-Silverstein, D., Barrett Connor, E., Milne, D., Nielsen, F., \& Gamst, A., et al. (2008). Plasma trace elements and cognitive function in older men and women: the Rancho Bernardo study. The Journal of Nutrition, Health \& Aging, 12(1), 22-7. [DOI:10.1007/ BF02982160] [PMID] [PMCID]

Lin, T., Liu, T., Lin, Y., Yan, L., Chen, Z., \& Wang, J. (2017). Comparative study on serum levels of macro and trace elements in schizophrenia based on supervised learning methods. Journal of Trace Elements in Medicine and Biology, 43, 202-8. [DOI:10.1016/J. JTEMB.2017.03.010] [PMID]

Liu, T., Lu, Q. B., Yan, L., Guo, J., Feng, F., Qiu, J., \& Wang, J. (2015). Comparative Study on Serum Levels of 10 Trace Elements in Schizophrenia. PloS One, 10(7), e0133622. [DOI:10.1371/journal. pone.0133622] [PMID] [PMCID]

Mazhari, S., Parvaresh, N., Eslami Shahrbabaki, M., Sadeghi, M. M., Nakhaee, N., \& Keefe, R. S. E. (2014). Validation of the Persian version of the Brief Assessment of Cognition in Schizophrenia in patients with schizophrenia and healthy controls. Psychiatry and Clinical Neurosciences, 68(2), 160-6. [DOI:10.1111/pcn.12107] [PMID]

Nechifor, M., Vaideanu, C., Palamaru, I., Borza, C., \& Mindreci, I. (2004). The influence of some antipsychotics on erythrocyte magnesium and plasma magnesium, calcium, copper and zinc in patients with paranoid Schizophrenia. Journal of the American College of Nutrition, 23(5), 549S-51S. [DOI:10.1080/07315724.2004.1071940 1] [PMID]

Ordak, M., Matras, J., Muszynska, E., Nasierowski, T., \& Bujalska-Zadrozny, M. (2017). Magnesium in schizophrenia. Pharmacological Reports, 69(5), 929-934. [DOI:10.1016/j. pharep.2017.03.022] [PMID]

Osredkar, J., \& Sustar, N. (2011). Copper and zinc, biological role and significance of copper/zinc imbalance. Journal of Clinical Toxicology, 3(2161), 0495. [DOI:10.4172/2161- 0495.S3-001]
Rahman, M. A., Azad, M. A. K., Hossain, M. I., Qusar, M. M. A. S., Bari, W., \& Begum, F., et al. (2009). Zinc, manganese, calcium, copper, and cadmium level in scalp hair samples of schizophrenic patients. Biological Trace Element Research, 127(2), 102-8. [DOI:10.1007/s12011-008-8230-8] [PMID]

Rahman, M. K., Rahman, F., Rahman, T., \& Kato, T. (2009). Dopamine- $\beta$-Hydroxylase (DBH), its cofactors and other biochemical parameters in the serum of neurological patients in Bangladesh. International Journal of Biomedical Science: IJBS, 5(4), 395-401. [DOI:10.1016/j.ijcard.2009.09.092] [PMID]

Ruljancic, N., Mihanovic, M., Cepelak, I., \& Bakliza, A. (2013) Platelet and serum calcium and magnesium concentration in suicidal and non-suicidal schizophrenic patients. Psychiatry and Clinical Neurosciences, 67(3), 154-9. [DOI:10.1111/ pcn.12038] [PMID]

Saha, S., Chant, D., Welham, J., \& McGrath, J. (2005). A Systematic Review of the Prevalence of Schizophrenia. PLoS Medicine, 2(5), e141. [DOI:10.1371/journal.pmed.0020141] [PMID] [PMCID]

Salustri, C., Barbati, G., Ghidoni, R., Quintiliani, L., Ciappina, S., \& Binetti, G., et al. (2010). Is cognitive function linked to serum free copper levels? A cohort study in a normal population. Clinical Neurophysiology, 121(4), 502-507. [DOI:10.1016/j. clinph.2009.11.090] [PMID]

Sara, S. J. (2009). The locus coeruleus and noradrenergic modulation of cognition. Nature Reviews Neuroscience, 10(3), 211-23. [DOI:10.1038/nrn2573] [PMID]

Smorgon, C., Mari, E., Atti, A. R., Dalla Nora, E., Zamboni, P. F., \& Calzoni, F., et al. (2004). Trace elements and cognitive impairment: an elderly cohort study. Archives of Gerontology and Geriatrics, 38, 393-402.. [DOI:10.1016/j.archger.2004.04.050] [PMID]

Tang, S., Yao, B., Li, N., Lin, S., \& Huang, Z. (2018). Association of dopamine beta- hydroxylase polymorphisms with Alzheimer's disease, Parkinson's disease and schizophrenia: Evidence based on currently available loci. Cellular Physiology and Biochemistry, 51(1), 411-28. [DOI:10.1159/000495238] [PMID]

Tripathi, A., Kar, S. K., \& Shukla, R. (2018). Cognitive deficits in schizophrenia: understanding the biological correlates and remediation strategies. Clinical Psychopharmacology and Neuroscience, 16(1), 7-17. [DOI:10.9758/cpn.2018.16.1.7] [PMID] [PMCID]

van Os, J., \& Kapur, S. (2009). Schizophrenia. The Lancet, 374(9690), 635-45. [DOI:10.1016/S0140-6736(09)60995-8]

Vendelboe, T. V., Harris, P., Zhao, Y., Walter, T. S., Harlos, K., \& El Omari, K., et al. (2016). The crystal structure of human dopamine $\beta$-hydroxylase at $2.9 \AA$ resolution. Science Advances, 2(4), e1500980. [DOI:10.1126/sciadv.1500980] [PMID] [PMCID]

Vidović, B., Đorđević, B., Milovanović, S., Škrivanj, S., Pavlović, Z., \& Stefanović, A., et al. (2013). Selenium, zinc, and copper plasma levels in patients with schizophrenia: Relationship with metabolic risk factors. Biological Trace Element Research, 156(1-3), 22-8. [DOI:10.1007/s12011-013-9842-1] [PMID]

Woods, S. W. (2003). Woods, S. W. (2003). Chlorpromazine equivalent doses for the newer atypical antipsychotics. The Journal of Clinical Psychiatry, 64(6), 663-67. [DOI:10.4088/JCP. v64n0607] [PMID] 
Yanik, M., Kocyigit, A., Tutkun, H., Vural, H., \& Herken, H. (2004). Plasma manganese, selenium, zinc, copper, and iron concentrations in patients with schizophrenia. Biological Trace Element Research, 98(2), 109-18. [DOI:10.1385/BTER:98:2:109]

Zhou, G., Ji, X., Cui, N., Cao, S., Liu, C., \& Liu, J. (2015). Association between serum copper status and working memory in schoolchildren. Nutrients, 7(9), 7185-96. [DOI:10.3390/ nu7095331] [PMID] [PMCID] 
This Page Intentionally Left Blank 


\title{
Research Paper: Lexical Access in Persian Speaking Children With and Without Specific Language Impairment
}

\author{
Fatemeh Hassanati $^{1}$ (D), Zahra Sadat Ghoreishi ${ }^{1^{*}}$ (D), Reza Nilipour ${ }^{1}$ (D), Abbas Pourshahbaz ${ }^{2}$ (D), Mohammad Momenian ${ }^{3}$ (D)
}

1. Department of Speech Therapy, School of Rehabilitation Sciences, University of Social Welfare and Rehabilitation Sciences, Tehran, Iran 2. Department of Clinical Psychology, School of Behavioral Sciences, University of Social Welfare and Rehabilitation Sciences, Tehran, Iran.

3. Laboratory for Communication Science, Division of Speech and Hearing Sciences, Faculty of Education, University of Hong Kong, Hong Kong, China.

\begin{tabular}{|l|l|l|l}
\hline $\begin{array}{c}\text { Use your device to scan } \\
\text { and read the article online }\end{array}$ & $\begin{array}{l}\text { Crtation: Hassanati, F., Ghoreishi, Z. S., Nilipour, R., Pourshahbaz, A., \& Momenian, M. (2020). Lexical Access in Persian } \\
\text { Speaking Children With and Without Specific Language Impairment. Basic and Clinical Neuroscience, 11(5), 659-668. http:// } \\
\text { dx.doi.org/10.32598/bcn.9.10.110 }\end{array}$ \\
dol'http://dx.doi.org/10.32598/bcn.9.10.110
\end{tabular}

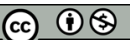

Article info:

Received: 28 Sep 2018

First Revision: 10 Oct 2018

Accepted: 01 Jan 2019

Available Online: 01 Sep 2020

Keywords:

Child, Data accuracy, Reaction time, Specific language impairment, Word processing

\section{A B S T RA C T}

Introduction: Word retrieval problems are among the limitations observed in children with specific language impairment during the initial schooling years. These restrictions are predictive of reading problems and poor performance at school. Additionally, studies on lexical access in Persian speaking children are scarce. Therefore, this study aimed to investigate and compare naming accuracy and latency in children with and without specific language impairment.

Methods: Twenty 7-9-year-old children with specific language impairment and 20 agematched peers were recruited as the study participants. They were requested to name the 128 black and white line-drawing pictures from a Persian picture naming set for children, as rapidly as possible. We compared the effects of psycholinguistic variables on naming latency in the explored children with and without specific language impairment.

Results: Linear mixed-effects modeling presented an interaction between the research groups and the psycholinguistic variables. Significant main effects were found for name agreement $(\mathrm{P} \leq 0.00)$ and the age of acquisition $(\mathrm{P}=0.05)$ in children with typical language development; significant effects for name agreement $(\mathrm{P} \leq 0.00)$ and $\log$ frequency $(\mathrm{P} \leq 0.00)$ were revealed in children with specific language impairment.

Conclusion: The obtained models indicated that psycholinguistic factors could differently affect the naming latency in children with and without specific language impairment. Factors that may have accounted for the findings are discussed in this paper.

\section{* Corresponding Author:}




\section{Highlights}

- The knowledge of word-finding abilities in children with specific language impairment could lead to choosing the best strategy for improving lexical access.

- The psycholinguistic variables should be controlled to achieve the best result for improving lexical access.

\section{Plain Language Summary}

Word finding difficulties are continuous problems in specific language impairment, which can affect literacy skills. The psycholinguistic variables could impact word-finding skills. Accordingly, therapists should be aware of psycholinguistic variables effective for the assessment and treatment of naming in children with specific language impairment. The obtained results suggested the significant impact of name agreement and word frequency on picture naming skills in the studied children. These results could help with the best planning of assessment and treatment of children with specific language impairment

\section{Introduction}

pecific Language Impairment (SLI) is a developmental disorder. In this condition, a child presents remarkable problems in language acquisition despite normal non-verbal intelligence and sensory ability (Leonard, 2014). This impairment is not homogenous and has a pattern, i.e. not observed in disorders with known causes, such as intellectual disability, autism, physical or neural impairments, and general learning disabilities. SLI includes a broad spectrum of deficits in various language aspects (Verhoeven \& van Balkom, 2003). There exists a large body of literature on morphosyntactic, phonological, and pragmatic complications in children with SLI; however, there is a dearth of research on the mental lexicon, especially word retrieval problems (Van der Lely \& Ullman, 2001; Bortolini \& Leonard, 2000; McGregor \& Appel, 2002).

When individuals learn new words, they store these words in their lexicon for later retrieval. Word retrieval significantly affects language processing and cognitive development (Messer \& Dockrell, 2006). Additionally, 25\% of children with language impairment encounter word-finding problems (Dockrell, Messer, \& George, 2001). Such conditions are significant predictors of reading disorders and poor educational performance in school-aged children (Wolf \& Segal, 1992). Numerous studies on children with SLI revealed that they name pictures more slowly and less accurately, compared to their counterparts with Typical Language Development (TLD) (Kambanaros \& Grohmann, 2010; Lahey \& Edwards, 1999; Leonard, Nippold, Kail, \& Hale, 1983; Miller, Kail, Leonard, \& Tomblin, 2001). Miller et al. (2001) found that children with SLI performed more slowly than those with TLD in all linguistic and nonlinguistic tasks; however, the speed of performance was higher in them, than that of the children with other language impairments.

Psycholinguistic variables are an essential determinant of the naming latency and accuracy, such as name agreement, the Age of Acquisition (AoA), and so on (Cycowicz, Friedman, Rothstein, \& Snodgrass, 1997; D’Amico, Devescovi, \& Bates, 2001; Masterson, Druks, \& Gallienne, 2008; Newman \& German, 2002). For instance, name agreement influences lemma selection (Alario et al., 2004; Bakhtiar, Nilipour, \& Weekes, 2015; Cycowicz, et al., 1997). Furthermore, AoA and word frequency impact lexical selection (Alario, et al., 2004). Words with highfrequency of occurrence and those with early AoA are retrieved more quickly than the rest (German \& Newman, 2004). Besides, numerous studies indicated that visual complexity influenced memory performance (Cycowicz, et al., 1997). More visually complex pictures require a longer time for retrieving the relevant concepts (Dimitropoulou, Dunabeitia, Blitsas, \& Carreiras, 2009). However, familiarity has an opposite effect, compared to visual complexity, in this respect. Rated familiarity influences the stages of conceptual activation (Alario, et al., 2004). There are some variations in the effects of psycholinguistic features on naming skills in different cultures. For example, some common objects in the USA, including animals and vegetables, seem to be unrecognized in East Asia (Yoon et al., 2004). Therefore, due to the structural and cultural characteristics of different languages, we selected the Persian language for this study.In the Persian language, most studies on children with SLI have focused on the assessment of syntax, morphology, and cognition (Ahadi, Nilipour, Rovshan, Ashayeri, \& Jalaie, 2014; Foroodi Nejad, 2011; Maleki 
Shahmahmood, Soleymani, \& Faghihzade, 2011). In the lexical domain, Mohammadi, Nilipour, Sima Shirazi, \& Rahgozar (2011) compared word (noun) definition skill between children with and without SLI. Accordingly, they detected significantly different scores in the content and structure aspects of the word definition skill between the study groups. Moreover, Persian speaking children with and without SLI presented a significant difference in word definition by labeling specific categories domain (Mohammadi, Nilipour, Sima Shirazi, \& Rahgozar, 2014). Except for these few studies on the lexical access domain, there are no investigations on lexical access; especially regarding the effect of psycholinguistic variables on naming skills in Persian speaking children with SLI.

The present study aimed to compare the picture-naming accuracy and latency between children with and without SLI. Furthermore, we attempted to determine the effect of psycholinguistic factors on naming latency using Linear Mixed Effect (LME) modeling in schoolaged children with and without SLI. This is the first report concerning the effect of psycholinguistic factors on confrontation naming tasks among Persian speaking children with and without SLI.

\section{Materials and Methods}

Twenty children with SLI aged 7-9 years (females $=8$, males $=12$; mean age $=8.1 \mathrm{y}$ ) and 20 children with TLD, aged 7-9 years $($ females $=8$, males $=12$; mean age $=8.3 \mathrm{y}$ ), as the control group, participated in this study. All research participants were monolingual Persian-speaking children. To select the TLD children, all children were assessed by a Speech-Language Pathologist (SLP) via an informal assessment. Raven's Colored Progressive Matrices Test (Karami, 2016) was performed among the study subjects. The investigated children's parents completed a questionnaire, containing demographic, medical, social, educational, and developmental history. We removed the children (from both study groups) with a serious vision problem.

The explored children with SLI were from the middle sociocultural class, studying in public schools. These children were selected from speech therapy clinics in Tehran City, Iran. The children were screened according to their SLP's opinions and parents' questionnaire data. There is a lack of a proper standardized test for school-aged children that could be considered a gold standard in Iran. Thus, we used clinical judgment as the reference standard to diagnose children with SLI (Kazemi \& Saeednia, 2017). Two qualified SLPs (the first author of this paper and another SLP who had clinical experience with children) examined the children. They used clinical examinations and considered some di- agnostic criteria, as follows: no history of communicative, phonological, and neurological problems; no motor speech disorders in informal oral assessments; no auditory problems revealed in the pure tone audiometry test; nonverbal Intelligence Quotient (IQ) to range within normal scope per Raven's Colored Progressive Matrices Test (Karami, 2016); and the presence of language problem diagnosis using the below scales:

Test of Language Development (TOLD-P:3): Its normalized information is available for children in Persian (Hasanzadeh \& Minaii, 2002).

Persian Test of SLI: This test comprises three criteria for assessment; the determination of the percentage score of language disorder in children with SLI, compared to agematched peers; the assessment of specific language features that have been impaired in children with SLI, and the determination of individual profile and severity of language impairment (Nilipour, 2002). This test's internal consistency was measured using Cronbach's alpha coefficient. Cronbach's alpha coefficient for this test was calculated as 0.90 for 60 children aged 5-10 years old (Nilipour, KarimiJavan, \& Ghoreishi, 2017). If the child was diagnosed with SLI by both SLPs, then he/she was included in this study. Eventually, 20 children were diagnosed with SLI. We received children's parents' permission for study participation by signing an informed consent form. The Ethics Committee code of this research was issued as IR.USWR. REC.1394.223.

The picture naming set consists of 128 simple black and white pictures. The picture naming set includes information on the frequency of name agreement, rated familiarity, visual complexity, AoA (for more information see Hassanati, Nilipour, Ghoreishi, Pourshahbaz \& Momenian, 2017), and word frequency (i.e. obtained from a standardized Persian written corpus; then it was transformed into log-frequency value).Table 1 shows the normative data of picture naming set in the studied Persian-speaking children.

Each explored child was tested individually in several sessions. In the initial three sessions, the diagnostic assessments were performed by SLPs. In the case of meeting the diagnostic criteria of SLI, a picture-naming task was performed in the final session.

The picture naming set was presented by DMDX software (Forster \& Forster, 2003). Each child was seated in front of a laptop connected to a microphone. The child was instructed to name the pictures quickly, with one word, without using extra words or voices. To make sure the child was prepared for the test and the instructions were clear enough, 
3-5 experimental pictures were illustrated to the child. The responses were recorded by DMDX and written by the examiner. Each picture was presented to the child for $5000 \mathrm{~ms}$ according to the pilot study and a previous study in children (D'Amico, et al., 2001). A time interval of $1000 \mathrm{~ms}$ was considered between presenting the two pictures. The pictures were randomly provided in 4 blocks, with the breaks between the blocks. If the study participant could not respond in $5000 \mathrm{~ms}$, an error was recorded by DMDX. Furthermore, if the study participant answered incorrectly, these responses were excluded from reaction time.

In this study, the LME modeling method was used for investigating the effect of the model of psycholinguistic factors on naming latency (Bakhtiar \& Weekes, 2015; Nilipour, Bakhtiar, Momenian, \& Weekes, 2016; Van Assche, Duyck, Hartsuiker, \& Diependaele, 2009). LME modeling has several advantages over the classic statistical analyses. It includes the item and subject random effects, which leads to higher generalizability of the findings to larger populations and stimuli (Baayen, Davidson, \& Bates, 2008; Jaeger, 2008; Quene \& Van den Bergh, 2008). To our knowledge, this is the first study about the effect of psycholinguistic variables on naming latency in children with SLI in Persian using LME modeling. Furthermore, in this study, the lme4 package (https://cran.r-project. org/web/packages/lme4/) was applied in R software to analyze the data of children with and without SLI.

\section{Results}

Naming latency was only calculated for correct names. In the SLI group, $26.3 \%$ of the responses were incorrect and $8.12 \%$ were not recorded by the software, i.e. excluded from the analysis. We controlled the outliers in naming latency. Then, the normality of the obtained data was examined by the Shapiro-Wilk test. Table 2 presents the naming latency and accuracy in children with and without SLI. The correlation between the reaction time scores of the randomly selected sample (TLD group) and the normative data of picture naming was $>0.75$, i.e. a high correlation $(\mathrm{P} \leq 0.05)$.

We used LME modeling to analyze the relationship between picture-naming latency and psycholinguistic factors in the study subjects. We followed the backward analysis procedure (Baayen, Davidson, \& Bates, 2008). Initially, we defined the full model, including all fixed variables along with the random variables of the subjects and items for all data. We found significant main effects for name agreement and AoA in the TLD; we also detected the same impacts for name agreement and log frequency in the SLI.

Next, we individually removed the variables, i.e. not significant in the full model. This measure aimed to determine whether their exclusion from the model reduced the model fit using the Likelihood Ration Test (LRT). The TLD data suggested that removing familiarity $\left(\chi_{(1)}^{2}=0.17\right.$, $\mathrm{P}=0.67)$, complexity $\left(\chi_{(1)}^{2}=0.02, \mathrm{P}=0.88\right)$, and $\log$ frequency $\left(\chi_{(1)}^{2}=0.005, \mathrm{P}=0.93\right)$ would not reduce the model fit. Subsequently, we defined other models in which significant main effects from the full model were removed one by one.

Table1. The normative data of picture naming set in the studied Persian-speaking children

\begin{tabular}{ccccccc}
\hline Variables & Name Agreement (\%) & Familiarity & Complexity & AoA (Month) & Log Frequency & RT (ms) \\
\hline Mean \pm SD & $86.09 \pm 17.10$ & $3.67 \pm 1.23$ & $2.32 \pm 0.91$ & $27.22 \pm 5.85$ & $1.83 \pm 0.55$ & $1399.8 \pm 218.24$ \\
Max. & 100 & 5 & 4.60 & 42 & 4.34 & 1941 \\
Min. & 31 & 1.20 & 1.00 & 11.60 & 1.00 & 10.56 \\
\hline
\end{tabular}

NEUROSCIENCE

Table 2. Mean(SD) scores of the naming latency of accurate responses in the picture naming set

\begin{tabular}{|c|c|c|c|c|}
\hline \multirow{2}{*}{ Groups of Children } & \multicolumn{2}{|c|}{ Mean $\pm S D$} & \multirow{2}{*}{$T$} & \multirow{2}{*}{ Df } \\
\hline & TLD & SLI & & \\
\hline Naming Latency (ms) & $1328.10 \pm 122.71$ & $1567.41 \pm 117.43$ & $-4.32 *$ & 36 \\
\hline Correct Answers (\%) & 85.2 & 73.7 & $4.07^{*}$ & \\
\hline
\end{tabular}

* The significance level is 0.05; * Independent Samples t-test; df: Degree of Freedom 
Table 3. A summary of LME models of RTs in the TLD group

\begin{tabular}{|ccccc}
\hline Fixed Effects & Estimate & Std. Error & t & P \\
\hline Intercept & 1813.93 & 180.26 & 10.06 & 0.001 \\
\hline name agreement & -8.36 & 1.54 & -5.43 & 0.001 \\
\hline AoA & 8.41 & 3.32 & 2.52 & 0.05 \\
\hline Random effects & Variance & Correlation & & \\
\hline Random effects & Variance & Correlation & & \\
\hline Items (Intercept) & 18627.64 & & \\
\hline Subj (Intercept) & 197187.63 & & -0.38 \\
\hline Subj (name agreement slope) & 22.28 & -0.90 & \\
\hline Subj (AoA slope) & 61.15 & -0.01 & \\
\hline Residual & 147052.70 & & \\
\hline
\end{tabular}

NEUR SCIENCE

Table 4. The summary of LME models of RTs in the SLI group

\begin{tabular}{ccccc}
\hline Fixed Effects & Estimate & Std. Error & t & P \\
\hline Intercept & 2806.50 & 169.40 & 16.56 & 0.001 \\
\hline name agreement & -10.87 & 1.78 & -6.10 & 0.001 \\
\hline Log frequency & -152.13 & 43.05 & -3.53 & 0.001 \\
\hline Random effects & Variance & & & \\
\hline Random effects & Variance & & \\
\hline Items (Intercept) & 40747 & & \\
\hline Subj (Intercept) & 35601 & & \\
\hline Residual & 245025 & & \\
\hline
\end{tabular}

NEUR SCIENCE

The related results revealed that excluding $\operatorname{AoA}\left(\chi_{(1)}^{2}=7.33\right.$, $\mathrm{P}<0.01)$ and name agreement $\left(\chi_{(1)}^{2}=41.98, \mathrm{P}<0.001\right)$ from the general model significantly reduced the model fit. The same procedure was followed with the SLI. First, we removed those predictor variables, i.e. not significant in the full model. The relevant results indicated that removing complexity $\left(\chi_{(1)}^{2}=0.99, \mathrm{P}=0.31\right)$, familiarity $\left(\chi_{(1)}^{2}=1.49\right.$, $\mathrm{P}=0.22)$, and $\operatorname{AoA}\left(\chi_{(1)}^{2}=1.96, \mathrm{P}=0.160\right)$ did not reduce the model fit at all. However, the one-by-one exclusion of name agreement $\left(\chi_{(1)}^{2}=23.87, \mathrm{P}<0.001\right)$ and $\log$ frequency $\left(\chi_{(1)}^{2}=6.22, \mathrm{P}<0.05\right)$, significantly impacted the model fit.

Then, we tested whether excluding by-subject and byitem random intercepts from the models influenced their fitness to model. The step-by-step removal of by-subject intercept $\left(\chi_{(1)}^{2}=97.67, \mathrm{P}<0.001\right)$ and by-item intercept $\left(\chi_{(1)}^{2}=66.91, \mathrm{P}<0.001\right)$ significantly affected the model fit in the TLD data. We witnessed the same effect in the SLI data. After removing the by-subject intercept and comparing the new model with the full model, these data were statistically significant $\left(\chi_{(1)}^{2}=97.67, \mathrm{P}<0.001\right)$. Excluding the by-item intercept from the model revealed the same result $\left(\chi_{(1)}^{2}=75.39, \mathrm{P}<0.001\right)$.

The optimal available model for the TLD data was RT name agreement + AoA $+(1 \mid$ subjects $)+(1 \mid$ items $)$. Next, we tested whether adding by-subject random slopes for significant effects could improve the model fit. Adding name 
agreement $\left(\chi_{(2)}^{2}=42.41, \mathrm{P}<0.001\right)$ and $\operatorname{AoA}\left(\chi_{(2)}^{2}=29.24\right.$, $\mathrm{P}<0.001)$ as by subject's random slopes significantly improved the model fit for the TLD data. The forwarding stepby-step analysis findings for the SLI data revealed the following model as the best one: RT $\sim$ name agreement $+\log$ frequency $+(1 \mid$ subjects $)+(1 \mid$ item $)$. We added by-subject random slopes for significant effects in the next stage. Including name agreement $\left(\chi_{(1)}^{2}=1, \mathrm{P}=1\right)$ and $\log$ frequency $\left(\chi_{(1)}^{2}=0.50, \mathrm{P}=0.47\right)$ failed to improve the model fit.

Based on the mentioned comparisons of the results, we proposed the following model for the TLD data (Table 3): $\mathrm{RT} \sim$ name agreement $+\mathrm{AoA}+(1+$ name agreement + AoA $\mid$ subjects $)+(1 \mid$ items $)$, and the following one for the SLI data (Table 4$):$ RT $\sim$ name agreement $+\log$ frequency $+(1 \mid$ subjects $)+(1 \mid$ items $)$.

\section{Discussion}

The present study aimed to determine the picture-naming accuracy and latency as well as the effect of psycholinguistic factors on naming latency in 7-9-year-old children with and without SLI. The obtained data indicated that children with SLI had less accurate and slower naming speed, compared to their age-matched peers. This finding was consistent with those of the previous studies (Lahey \& Edwards, 1996; Montgomery, 2002; Windsor \& Hwang, 1999; Hassanati, Nilipour, Ghoreishi, Pourshahbaz \& Momenian, 2018). Leonard et al. (1983) concluded that children with SLI performed more quickly than younger languagematched peers and more slowly than age-matched counterparts. An explanation of such findings could be a different language development and deficit in the semantic representation of words in children with SLI (Leonard, et al., 1983). Some scholars believed that the size of expressive vocabulary could determine the level of word retrieval success (Leonard, et al., 1983; Sheng, 2010). Additionally, the reason for the slower naming times of the languageimpaired children could be a limitation in word retrieval or storage limitation) Messer, \& Dockrell, 2006).

LME modeling method was used for exploring the model of psycholinguistic factors' effect on naming latency. The models obtained in children with and without SLI indicated that name agreement significantly impacted naming latency. This effect may be due to the competition between the target name and incorrect responses, as well as that between the target name and its alternative names. Selecting the target name among numerous alternative names in the mental lexicon requires a longer time (Alario et al., 2004; Cycowicz, et al., 1997).
Furthermore, the obtained model in children with TLD indicated that AoA affects the naming latency in them. This result was consistent with those of the previous studies (Cycowicz, et al., 1997; D'Amico, et al., 2001; Newman \& German, 2002). Newman and German (2005) concluded that the words acquired at the lower ages are more easily retrieved than those acquired later (Newman \& German, 2005). When the words were acquired earlier, there were more connections between the semantic and phonological components of the words; thus, they have retrieved more accurately (Gershkoff-Stowe \& Smith, 1997). According to the growing network model, the words acquired earlier have higher connections and present a central position in the semantic network (Steyvers \& Tenenbaum, 2005).

The present model obtained for children with SLI indicated that word frequency influenced the latency of picture naming. This model indicated that children with SLI were significantly better at naming high-frequency words, than naming low-frequency words. Multiple studies indicated that the children with and without various language disorders encounter further problems in naming the words with lower frequency (Cycowicz, et al., 1997; D'Amico, et al., 2001; Kambanaros \& Grohmann, 2010; Mainela-Arnold \& Evans, 2005; Newman \& German, 2002). Leonard et al. (1983) concluded that high-frequency words have more connections. Besides, they have a stronger representation in the mental lexicon that requires less time to access (Leonard, et al., 1983). Moreover, the frequency might affect the connections between various representations, especially lemma and lexeme (Alario, et al., 2004; Barry, Hirsh, Johnston, \& Williams, 2001; Barry, Morrison, \& Ellis, 1997). Words with high-frequency occurrences have a lower activation threshold of a word, thereby facilitating word retrieval (Dewhurst, Hitch, \& Barry, 1998; Sheng, 2007).

AoA seems to have a universal significant effect on timed picture naming across languages in impaired and unimpaired adult speakers (Alario, et al., 2004; Bakhtiar, Nilipour, \& Weekes, 2013; Bates et al., 2003; Nilipour, Bakhtiar, Momenian, \& Weekes, 2016); as well as in children with TLD and word-finding difficulties (Cycowicz, et al., 1997; D'Amico, et al., 2001; German \& Newman, 2004; Newman \& German, 2002). However, we observed no effect of AoA in children with SLI. It is necessary to note that most studies on naming skills have been conducted on adults or children with TLD. This result could be explained by some reasons. One of the possible reasons for not observing the effect of AoA in children with SLI could be the general delay in vocabulary acquisition and the inefficiency of lexical access in children with SLI (Dockrell \& Messer, 2004). Children with SLI acquire the words later in a limited time (usually with practice) (Sheng, 2010); accord- 
ingly, the effect of AoA may not be observed in them, like children with TLD. Furthermore, lexical items are poorly differentiated in their semantic-lexical representations and these representations may not be well organized. However, it is necessary to examine the effect of AoA on naming skills in different languages by various methods to reach a definite result.

\section{Conclusion}

Generally, this study could help to better understand this important aspect of children's language use. The present study was the first to apply LME modeling of the effect of psycholinguistic factors on naming latency in children with and without SLI. The obtained models indicated that such factors could differently impact the naming latency in children with and without SLI. Such differences may be due to the delay in language acquisition such as lexical access in children with SLI. These results could be useful for adequate assessment and intervention in language-impaired children. Future studies may focus on the effect of other psycholinguistic factors, such as neighborhood density in the naming skill of children. However, further studies are required on more subjects in different languages by highlyaccurate statistical approaches, like LME modeling.

\section{Ethical Considerations}

\section{Compliance with ethical guidelines}

This study was approved by the Ethics Committee of the University of Social Welfare and Rehabilitation Science (IR.USWR.REC.1394.223). Written informed consent was obtained from the parents of study participants.

\section{Funding}

This research was supported by the Cognitive Sciences and Technological Council (2340).

\section{Authors' contributions}

Conceptualization: Fatemeh Hassanati, Reza Nilipour, Zahra Sadat Ghoreishi, Mohammad Momenian; Methodology: Fatemeh Hassanati, Reza Nilipour, Zahra Sadat Ghoreishi, Mohammad Momenian; Writing-original draft, investigation: All author; Writing - review \& editing: Fatemeh Hassanati, Reza Nilipour, Zahra Sadat Ghoreishi; Funding acquisition: Fatemeh Hassanati, Reza Nilipour, Zahra Sadat Ghoreishi; Resources: Fatemeh Hassanati, Reza Nilipour, Zahra Sadat Ghoreishi, Mohammad Momenian; Analysis Fatemeh Hassanati, Mohammad Momenian; Supervision: Reza Nilipour, Zahra Sadat Ghoreishi.

\section{Conflict of interest}

The authors declared no conflicts of interest.

\section{References}

Ahadi, H., Nilipour, R., Rovshan, B., Ashayeri, H., \& Jalaie, S. (2014). [The perception and expression of verb morphology in bilinguals with specific language impairment (Persian)]. Audiology, 23(1), 62-9. https:/ / aud.tums.ac.ir/article-1-5124-fa.pdf

Alario, F. X., Ferrand, L., Laganaro, M., New, B., Frauenfelder, U. H., \& Segui, J. (2004). Predictors of picture naming speed. Behavior Research Methods, Instruments, E Computers, 36(1), 140-55. [DOI:10.3758/BF03195559] [PMID]

Baayen, R. H., Davidson, D. J., \& Bates, D. M. (2008). Mixedeffects modeling with crossed random effects for subjects and items. Journal of Memory and Language, 59(4), 390-412. [DOI:10.1016/j.jml.2007.12.005]

Bakhtiar, M., Nilipour, R., \& Weekes, B. S. (2013). Predictors of timed picture naming in Persian. Behavior Research Methods, 45(3), 834-41. [DOI:10.3758/s13428-012-0298-6] [PMID]

Bakhtiar, M., \& Weekes, B. (2015). Lexico-semantic effects on word naming in Persian: Does age of acquisition have an effect? Memory \& Cognition, 43(2), 298-313. [DOI:10.3758/ s13421-014-0472-4] [PMID]

Barry, C., Hirsh, K. W., Johnston, R. A., \& Williams, C. L. (2001). Age of acquisition, word frequency, and the locus of repetition priming of picture naming. Journal of Memory and Language, 44(3), 350-75. [DOI:10.1006/jmla.2000.2743]

Barry, C., Morrison, C. M., \& Ellis, A. W. (1997). Naming the Snodgrass and Vanderwart pictures: Effects of age of acquisition, frequency, and name agreement. The Quarterly Journal of Experimental Psychology: Section A, 50(3), 560-85. [DOI:10.1080/027249897392026]

Bates, E., D’Amico, S., Jacobsen, T., Székely, A., Andonova, E. Bishop, D. V., \& Norbury, C. F. (2002). Exploring the borderlands of autistic disorder and specific language impairment: A study using standardised diagnostic instruments. Journal of Child Psychology and Psychiatry, 43(7), 917-29. [DOI:10.1111/1469-7610.00114] [PMID]

Bortolini, U., \& Leonard, L. B. (2000). Phonology and children with specific language impairment: Status of structural constraints in two languages. Journal of Communication Disorders, 33(2), 131-150. [DOI:10.1016/S0021-9924(99)00028-3]

Cycowicz, Y. M., Friedman, D., Rothstein, M., \& Snodgrass, J. G. (1997). Picture naming by young children: Norms for name agreement, familiarity, and visual complexity. Journal of Experimental Child Psychology, 65(2), 171-237. [DOI:10.1006/ jecp.1996.2356] [PMID]

D’Amico, S., Devescovi, A., \& Bates, E. (2001). Picture naming and lexical access in Italian children and adults. Journal of Cognition and Development, 2(1), 71-105. [DOI:10.1207/ S15327647JCD0201_4]

Dewhurst, S. A., Hitch, G. J., \& Barry, C. (1998). Separate effects of word frequency and age of acquisition in recognition and 
recall. Journal of Experimental Psychology: Learning, Memory, and Cognition, 24(2), 284-98. [DOI:10.1037/0278-7393.24.2.284]

Dimitropoulou, M., Dunabeitia, J. A., Blitsas, P., \& Carreiras, M. (2009). A standardized set of 260 pictures for Modern Greek: Norms for name agreement, age of acquisition, and visual complexity. Behavior Research Methods, 41(2), 584-9. [DOI:10.3758/BRM.41.2.584] [PMID]

Dockrell, J. E., \& Messer, D. (2004). Lexical acquisition in the early school years. Language Development Across Childhood and Adolescence, 3, 35-52. [DOI:10.1075/tilar.3.06doc]

Dockrell, J. E., Messer, D., \& George, R. (2001). Patterns of naming objects and actions in children with word finding difficulties. Language and Cognitive Processes, 16(2-3), 261-86. [DOI:10.1080/01690960042000030]

Foroodi Nejad, F. (2011). [Towards the identification of linguistic characteristics of specific language impairment in Persian (Persian)]. [PhD. thesis]. Edmonton, Alberta: University of Alberta; 2011

Forster, K. I., \& Forster, J. C. (2003). DMDX: A windows display program with millisecond accuracy. Behavior Research Meth ods, Instruments, \& Computers, 35(1), 116-24. [DOI:10.3758/ BF03195503] [PMID]

German, D. J., \& Newman, R. S. (2004). The impact of lexical factors on children's word-finding errors. Journal of Speech, Language, and Hearing Research, 47(3), 624-36. [DOI:10.1044/10924388(2004/048)]

Gershkoff-Stowe, L., \& Smith, L. B. (1997). A curvilinear trend in naming errors as a function of early vocabulary growth. Cognitive Psychology, 34(1), 37-71. [DOI:10.1006/cogp.1997.0664] [PMID]

Hasanzadeh, S., Minaei, A. (2002). [Adaptation and standardization of the test of TOLD-P: 3 for Farsi - speaking children of Tehran (Persian)]. Journal of Exceptional Children, 1(2), 119-34. http://joec.ir/article-1-505-fa.html

Hassanati, F., Nilipour, R., Ghoreishi, Z. S., Pourshahbaz, A., \& Momenian, M. (2018). [Picture naming in children with and without specific language impairment: An error analysis study (Persian)]. Iranian Rehabilitation Journal, 16(3), 289-96 [DOI:10.32598/irj.16.3.289]

Hassanati, F., Nilipour, R., Ghoreishi, Z., Pourshahbaz, A. \& Momenian, M. (2017). [Predictors of Timed Picture Naming in Persian Speaking Children (Persian)]. International Journal of Children and Adulscents, 3(4), 13-21. http://ijca.iums.ac.ir/ article-1-101-fa.html

Jaeger, T. F. (2008). Categorical data analysis: Away from ANOVAs (transformation or not) and towards logit mixed models. Journal of Memory and Language, 59(4), 434-446. [DOI:10.1016/j. jml.2007.11.007] [PMID] [PMCID]

Kambanaros, M., \& Grohmann, K. K. (2010). Patterns of object and action naming in cypriot Greek children with SLI and WFDs. Paper presented at the $34^{\text {th }}$ Boston University Conference on Language Development. https://core.ac.uk/download/ pdf/267932244.pdf

Karami, A. (2016). Child inteligence measurement (Raven children's test) (Vol. 14 $\left.{ }^{\text {th }}\right)$. Tehran: Psychometric. https://www.ravansanji.com/product/1/
Kazemi, Y., \& Saeednia, S. (2017). The clinical examination of non-word repetition tasks in identifying Persian-speaking children with primary language impairment. International Journal of Pediatric Otorhinolaryngology, 93, 7-12. [DOI:10.1016/j.ijporl.2016.11.028] [PMID]

Lahey, M., \& Edwards, J. (1996). Why do children with specific language impairment name pictures more slowly than their peers? Journal of Speech, Language, and Hearing Research, 39(5), 1081-98. [DOI:10.1044/jshr.3905.1081] [PMID]

Lahey, M., \& Edwards, J. (1999). Naming errors of children with specific language impairment. Journal of Speech, Language, and Hearing Research, 42(1), 195-205. [DOI:10.1044/jslhr.4201.195] [PMID]

Leonard, L. B. (2014). Children with specific language impairment. Cambridge: MIT Press. [DOI:10.7551/mitpress/9152.001.0001]

Leonard, L. B., Nippold, M. A., Kail, R., \& Hale, C. A. (1983). Picture naming in language-impaired children. Journal of Speech, Language, and Hearing Research, 26(4), 609-15. [DOI:10.1044/ jshr.2604.609] [PMID]

Mainela-Arnold, E., \& Evans, J. L. (2005). Beyond capacity limitations: Determinants of word recall performance on verbal working memory span tasks in children with SLI. Journal of Speech, Language, and Hearing Research, 48(4), 897-909. [DOI:10.1044/1092-4388(2005/062)]

Maleki Shahmahmood, T., Soleymani, Z., \& Faghihzade, S (2011). [The study of language performances of Persian children with specific language impairment (Persian)]. Bimonthly Audiology-Tehran University of Medical Sciences, 20(2), 11-21. https://aud.tums.ac.ir/article-1-61-fa.pdf

Masterson, J., Druks, J., \& Gallienne, D. (2008). Object and action picture naming in three-and five-year-old children. Journal of Child Language, 35(2), 373-402. [DOI:10.1017/ S0305000907008549] [PMID]

McGregor, K. K., \& Appel, A. (2002). On the relation between mental representation and naming in a child with specific language impairment. Clinical Linguistics \& Phonetics, 16(1), 1-20. [DOI:10.1080/02699200110085034] [PMID]

Messer, D., \& Dockrell, J. E. (2006). Children's naming and word-finding difficulties: Descriptions and explanations. Journal of Speech, Language, and Hearing Research, 49(2), 309-24. [DOI:10.1044/1092-4388(2006/025)]

Miller, C. A., Kail, R., Leonard, L. B., \& Tomblin, J. B. (2001). Speed of processing in children with specific language impairment. Journal of Speech, Language, and Hearing Research, 44(2), 416-33. [DOI:10.1044/1092-4388(2001/034)]

Mohammadi, M., Nilipour, R., Sima Shirazi, T., \& Rahgozar, M (2011). [Semantic differences of definitional skills between persian speaking children with specific language impairment and normal language developing children (Persian)]. Journal of Rehabilitation, 12(2), 48-55. http://rehabilitationj.uswr. ac.ir/article-1-886-fa.html

Mohammadi, M., Nilipour, R., Sima Shirazi, T., \& Rahgozar, M. (2014). [Examining expression of lexical categories in Farsi- speaking children with specific language impairment (Persian)]. Speech and Language Pathology, 1(1). https:/ / www. magiran.com/paper $/ 1456800$ ?lang= 
Montgomery, J. W. (2002). Examining the nature of lexical processing in children with specific language impairment: Temporal processing or processing capacity deficit? Applied Psycholinguistics, 23(03), 447-470. [DOI:10.1017/S0142716402003077]

Nematzadeh, S., Dadras, M., \& DastjerdiKazemi, M. (2011). Persian core vocabulary based on Iranian children. Tehran: Madreseh.

Newman, R. S., \& German, D.J. (2002). Effects of lexical factors on lexical access among typical language-learning children and children with word-finding difficulties. Language and Speech, 45(3), 285-317. [DOI:10.1177/00238309020450030401] [PMID]

Newman, R. S., \& German, D. J. (2005). Life span effects of lexical factors on oral naming. Language and Speech, 48(2), 123-56. [D OI:10.1177/00238309050480020101] [PMID]

Nilipour, R. (2002). Persian test of specific language impairment Tehran: University of Social Welfare and Rehabilitation Sciences.

Nilipour, R., Bakhtiar, M., Momenian, M., \& Weekes, B. S (2016). Object and action picture naming in brain-damaged Persian speakers with aphasia. Aphasiology, 31(4), 1-18. [DOI:1 $0.1080 / 02687038.2016 .1234583]$

Nilipour, R., Karimi-Javan, G., \& Ghoreishi, Z. S. (2017). [Predictors of Quality of Speech and Slow Information Processing in Persian Children with Special Language Impairment (SLI) (Persian)]. Journal of Exceptional Children, 15(2), 67-77. http://joec.ir/article-1-75-fa.html

Quene, H., \& Van den Bergh, H. (2008). Examples of mixed-effects modeling with crossed random effects and with binomial data. Journal of Memory and Language, 59(4), 413-25. https:// doi.org/10.1016/j.jml.2008.02.002

Sheng, L. (2010). Lexical access and semantic organization in children with specific language impairment. Journal of Speech, Language, and Hearing Research, 53(1), 146-59. [DOI:10.1044/1092-4388(2009/08-0160)] [PMCID] [PMID]

Steyvers, M., \& Tenenbaum, J. B. (2005). Graph theoretic analyses of semantic networks: Small worlds in semantic networks. Cognitive Science, 29(1), 41-78. [DOI:10.1207/ s15516709cog2901_3] [PMID]

Van Assche, E., Duyck, W., Hartsuiker, R. J., \& Diependaele, K. (2009). Does bilingualism change native-language reading? Cognate effects in a sentence context. Psychological Science, 20(8), 923-7. [DOI:10.1111/j.1467-9280.2009.02389.x] [PMID]

Van der Lely, H. K., \& Ullman, M. T. (2001). Past tense morphology in specifically language impaired and normally developing children. Language and Cognitive Processes, 16(2-3), 177-217. [DOI:10.1080/01690960042000076]

Verhoeven, L., \& van Balkom, H. (2003). Classification of developmental language disorders: Theoretical issues and clinical implications: Psychology Press. [DOI:10.4324/9781410609021]

Windsor, J., \& Hwang, M. (1999). Testing the generalized slowing hypothesis in specific language impairment. Journal of Speech, Language, and Hearing Research, 42(5), 1205-1218. [DOI:10.1044/jslhr.4205.1205] [PMID]

Wolf, M., \& Segal, D. (1992). Word finding and reading in the developmental dyslexias. Topics in Language Disorders, 13(1), 51-65. [DOI:10.1097/00011363-199211000-00007]
Wolfus, B., Moscovitch, M., \& Kinsbourne, M. (1980). Subgroups of developmental language impairment. Brain and Language, 10(1), 152-171. [DOI:10.1016/0093-934X(80)90046-2]

Yoon, C., Feinberg, F., Luo, T., Hedden, T., Gutchess, A. H., \& Chen, H.Y. M., et al. (2004). A cross-culturally standardized set of pictures for younger and older adults: American and Chinese norms for name agreement, concept agreement, and familiarity. Behavior Research Methods, 36(4), 639-49. [DOI:10.3758/BF03206545] [PMID] 
This Page Intentionally Left Blank 


\title{
Research Paper: Larger Volume and Different Activation of the Brain in Response to Threat in Military Officers
}

\author{
Seyed Amir Hossein Batouli ${ }^{1}$ (i), Valiallah Saba ${ }^{t^{*}}$ (1)
}

1. Department of Radiology, Faculty of Paramedicine, AJA University of Medical Sciences, Tehran, Iran

\begin{tabular}{|c|c|}
\hline 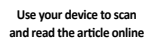 & \\
\hline 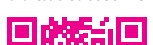 & ditation: Batouli, S. A. H., \& Saba, V. (2020). Larger Volume and Different Activation of the Brain in Response to Threat in \\
\hline 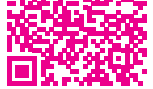 & dol'http://dx.doi.org/10.32598/bcn.9.10.160 \\
\hline
\end{tabular}

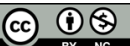

Article info:

Received: 13 Jul 2019

First Revision: 05 Aug 2019

Accepted: 15 Jan 2020

Available Online: 01 Sep 2020

Keywords:

Brain, Cognition, Military personnel, Magnetic

Resonance Imaging (MRI)

\section{A B S T RA C T}

Introduction: Military missions involve stressful and life-threatening situations; however, soldiers should have a healthy cognition on the battlefield despite their high-stress levels. This is an ability that should be gained during prior military training. Successful and influential training is suggested to be associated with structural and functional improvements of the brain

Methods: This study investigated the pattern of brain activation while observing videos relevant to life-threatening situations, in addition to brain structure. Accordingly, the obtained data were compared between 20 military members and 26 healthy controls. The study participants were all male, aged between 19 to 24 years, right-handed, studying BSc, and from the same socioeconomic status.

Results: The obtained data presented a larger volume in a total number of 1103 voxels of the brain (in 5 brain areas) in the military group. Furthermore, the military group suggested higher brain activation in the visual processing areas of the brain when observing real combat videos; however, this increment was mostly in the areas associated with motor processing and executive functions in the controls.

Conclusion: This study indicated that military training is associated with positive structural changes in the brain. Besides, it provided a different brain activation in response to stressful situations. These findings highlighted the importance of qualified military training.

\section{* Corresponding Author:}

Valiallah Saba, PhD.

Address: Department of Radiology, Faculty of Paramedicine, AJA University of Medical Sciences, Tehran, Iran.

Tel: +98 (21) 43822449

E-mail: vsaba@aut.ac.ir 


\section{Highlights}

- Military training is positively associated with brain structure alternations.

- Military training is associated with brain functionality.

- Military officers present a different brain activation in response to the threat.

\section{Plain Language Summary}

The army of a country is protecting the land, residents, property, and independence of that country. The condition of a battlefield is full of stress and life-threatening situations. Well, if an army member loses his attention and concentration during the battle, he may easily lose his life. In addition, that group of soldiers may have a lower chance to complete their mission. As a result, it is very important to know if an army member is ready for deployment and will be focused on his duties during the battle. In this study, we compared a group of young under-training army members, who had not been sent to any deployment, with a group of healthy controls. We aimed to understand if the military training in the army group was correlated with the structure and function of the brain; in other words, we expected that all those trainings suggest some associations with a healthier and more efficient brain in the army members. We observed larger volumes in some areas of the brain in the army group, i.e. expected, due to receiving high biopsychological trainings. Besides, the army group had less brain activation when observing real-combat, stressful movies. This finding reveals that they had a better regulation of their emotions against life-threatening situations. This study highlighted that the military training of our army members is efficient enough, to prepare them for deployment.

\section{Introduction}

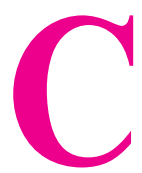

ognitive failure is prevalent in individuals under stress, which may disappear when the stress decreases (van Wingen et al., 2012); however, severe stress, like those experienced in a military mission, can longer and more severely lead to cognitive impairments. Accordingly, it influences the performance, cognitive behavior, and occupational functioning of the individuals ("Self-reported illness and health status among gulf war veterans: A population-based study", 1997). Stress is referred to as a state of threatened homeostasis in an organism, due to internal or external effects (Chrousos, 2009). The physiological response towards it would be the activation of the sympathetic nervous system and the Hypothalamic-Pituitary-Adrenal (HPA) axis (Steinbeis, Engert, Linz, \& Singer, 2015a), as well as enhanced cortisol level (Hellhammer, Wüst, \& Kudielka, 2009). This physiological response and the corresponding rises in arousal, vigilance, aggression, and attention, enable a behavioral response, called "fight-or-flight" (Steinbeis, Engert, Linz, \& Singer, 2015b).

Stress presents profound effects on human cognition (van Wingen et al., 2012); this includes not only making decisions and planning actions (Lerner \& Keltner, 2001), but also the memory and perception of sensory informa- tion (Phelps, 2004). Adverse influences of stress are also illustrated on the lack of time and place orientation (Ćosić et al., 2012), emotionality (Eiland \& Romeo, 2013), the structure of amygdalar neurons (McEwen, Nasca, \& Gray, 2015), enhanced anxiety-like behaviors (Padival, Blume, Vantrease, \& Rosenkranz, 2015), the atrophy of hippocampal cells (McEwen et al., 2015), and the executive functions (van Wingen et al., 2012). As a military example, the combat stress in the US army soldiers in Afghanistan suggested reduced midbrain activity and integrity, in association with compromised sustained attention, as well as reduced functional connectivity between the midbrain and prefrontal cortex (van Wingen et al., 2012).

Therefore, it is essential to empowering the soldiers to maintain their proper cognition and performance on the battlefield. Such measures enable them to protect themselves and complete their mission. One method to achieve this goal is through persistent military training and education. It has been suggested that military training does enable professional soldiers (even unconsciously) to control their brain activity and not being affected by threats (Ćosić et al., 2012). This ability is much weaker or unavailable in the general population. A mindfulness-based series of training also positively impacted the brain-behavior relationship in soldiers. Some examples included enhanced stress recovery, decreased plasma neuropeptide Y concentration, and attenuated 
brain activations in the insula and anterior cingulate. Emotion regulation assists individuals to tailor their emotional responses in a dynamic environment (Raio \& Phelps, 2015); however, stress exposure exerts detrimental effects on this ability among them. Mission success depends on the soldiers' ability to manage and leverage their emotions (Raio \& Phelps, 2015). Subsequently, it is vital for the soldiers to practice and gain the required skills to cognitively manage their emotions as a driving force of attention, motivation, and learning (Oden, Lohani, McCoy, Crutchfield, \& Rivers, 2015).

In addition to mental training, Physical Activity (PA) could positively influence the brain's cognitive abilities (Ang, Gomez-Pinilla, \& Gomez-Pinilla, 2007); this impact is to the extent that PA is described as the most efficient manner to have a healthy mind (van Praag, 2009). For example, aerobic exercise is observed to improve the efficiency of attentional (Kramer et al., 1999), executivecontrol (Colcombe \& Kramer, 2003), and learning (Perini, Bortoletto, Capogrosso, Fertonani, \& Miniussi, 2016) processes; a higher fitness level was reported to be associated with less cognitive decline in the elderly (Barnes, Yaffe, Satariano, \& Tager, 2003); with enhanced cognition, as well as improved learning and memory (Cotman \& Berchtold, 2002), attention, reaction time, and language (Snowden et al., 2011; Batouli \& Sisakhti, 2019), and with visuospatial, and other neurocognitive functions (Braskie et al., 2014). Additionally, resistance training improved associative memory performance and visual processing strategy (Liu-Ambrose, Nagamatsu, Voss, Khan, \& Handy, 2012). Besides, coordination training was associated with improved cognition (Niemann, Godde, \& Voelcker-Rehage, 2016). Exercise also reduces the incidence of stress-related disorders (Herrera et al., 2016), such as depression, post-traumatic stress disorder, and anxiety (Asmundson et al., 2013).

PA is positively correlated with the brain structure as well. A review study demonstrated that $>80 \%$ of the brain's gray matter is related to PA(Batouli \& Saba, 2017). The PAs included aerobic exercise, endurance, strength and balance training, weight press and stretching, as well as sports such as biking, running, martial arts, ball sports, golfing, and diving. That study indicated the substantial association of PA with the brain structure. Moreover, the relevant findings emphasized implementing PA in the programs related to improving mental abilities, including military training.

As a result, it is essential to evaluate the association between the biopsychological training of military officers and the alterations of their brain structure and function.
For this aim, this study acquired two groups of participants, consisting of military officers and controls. Both study groups were matched in terms of age, educational level, and socioeconomic status. We compared their brain structure, as well as their brain activations pattern while observing stressful movies of real combat. The current research findings could help evaluate the efficacy of our military training to prepare soldiers for deployment. Accordingly, we could save their lives and help them to complete their mission.

\section{Materials and Methods}

This study was performed per the Ethics Statement of Iran University of Medical Sciences (ethics approval code: IR.IUMS.REC.1395.899). All study participants declared their assent during the initial interview after being informed about the general purposes of the study. Accordingly, they provided signed informed consent forms on the test day. Forty-six right-handed male subjects were included in this study. Of them, 26 were healthy controls (Mean \pm SD age: $20.5 \pm 1.02$ years; age range: $19-22$ years), who were BSc students of Medicine, Nursing, or Radiology. The test group included 20 young military officers (Mean \pm SD age: $22.8 \pm 0.8$ years; age range: 20 - 24 years), who were also BSc students at an Iranian military educational institution. Besides, they had successfully passed the basic educational courses of the Iranian army, including heavy physical, martial, and military training, as well as the theoretical military educations. The two groups were selected from the same socioeconomic status; however, the military group members were older; therefore, all estimations were corrected for age.

The control group was not sedentary; however, heavy training and a few hours of physical exercise was a daily program of the military officers (for around 3 years). Thus, the amount of time and effort that the military group devotes to PA was higher than that of the controls. As evidence, the Mean \pm SD Body Mass Index (BMI) scores of the military and control groups were $21.4 \pm 1.89$ (17.7$24.8)$ and $23.1 \pm 3.22$ (17.5-30.1), respectively. The BMI measures of both research groups were in the healthy range; however, the lower BMI in the military group was statistically significant (Paired Samples t-test, $\mathrm{P}=0.047$ ).

The biopsychological health of the study participants was tested by a physician at the imaging center using our developed questionnaire, i.e. based on the inclusion/ exclusion criteria of the International Consortium for Brain Mapping (ICBM) (Mazziotta et al., 2009). The study participants were excluded due to any current or past chronic or acute neurological or internal conditions, 
medicine consumption, undergoing surgery, experiencing trauma; being overweight ( $>100 \mathrm{~kg}$ ); having a serious family history of any diseases; smoking or drug/ alcohol abuse; being claustrophobic, or having implants or any other metal objects in the body.

To assess the underlying emotional state of the participants, the Positive and Negative Affect Schedule (PANAS) test (Tran, 2013) was performed before conducting the Magnetic Resonance Imaging (MRI). As a result, the PANAS mean \pm SD scores (based on a 50 -point scale) of the military group was "positive $=38.1 \pm 4.3$ "; and "negative $=18.5 \pm 5.5$ "; for the control group, the same was "positive= 35.2 \pm 5.5 ", and "negative $=17.6 \pm 5.9$ ". The two groups did not differ in their negative or positive PANAS measures $(\mathrm{P}>0.05)$.

The functional MRI (fMRI) paradigm was designed to simulate a stressful and life-threatening situation for the study participants. For this aim, 5 conditions were randomly set up in a block-design paradigm using short web-downloaded video clips. The conditions were as follows: I) videos relevant to a European football league (football); II) short videos of real combat footage, without scenes relevant to murder, violence, or brutality (war-1); III) real footage of combat, with scenes relevant to murder and violence, but without brutality (war-2); IV) videos of combat, with murder, violence, and brutality (war-3); and V) the rest condition, which included a black cross on a white background (rest). Providing three types of war conditions was to assure that approximately all kinds of real-combat situations were presented to the research participants. The football videos were presented as neutral stimulations. The sample screenshots of the videos are provided in Figure 1.

The paradigm lasted 11:55 minutes. It comprised 6 rest conditions ( $24 \mathrm{~s}$ each), 3 football conditions ( $30 \mathrm{~s}$ each), 3 videos of war- 1 condition (30-50s each), 2 videos of war- 2 condition ( 30 s each), and 5 videos of war- 3 condition (34-60s each). The conditions were initially randomly distributed; then, the same paradigm was presented to all study participants. The videos and their sounds were presented to the research participants during the MRI scan using a goggle $(800 \times 600$-pixel resolution in a 0.25 square area and refresh rate of up to $85 \mathrm{~Hz}$ ) and an earphone $(30 \mathrm{~dB}$ noise-attenuating headset with $40 \mathrm{~Hz}$ to $40 \mathrm{kHz}$ frequency response), i.e. appropriate to up to 4.7T magnetic fields (VisuaStim, The Pennsylvania State University, USA).

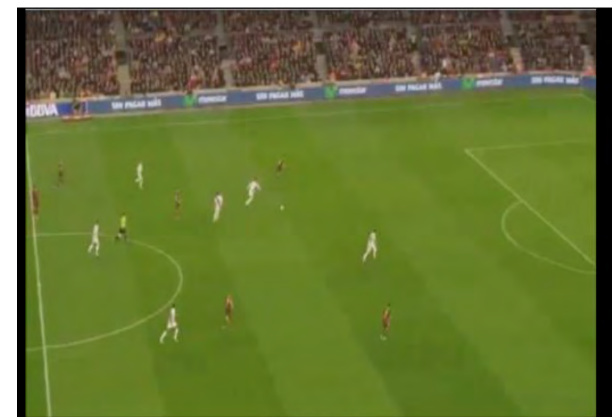

Football

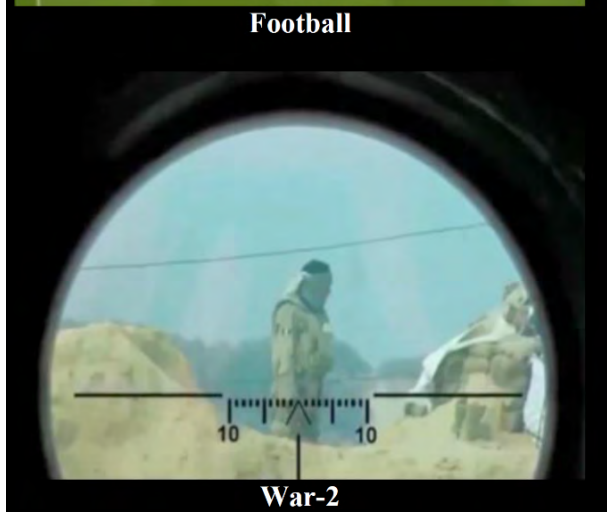

War-2
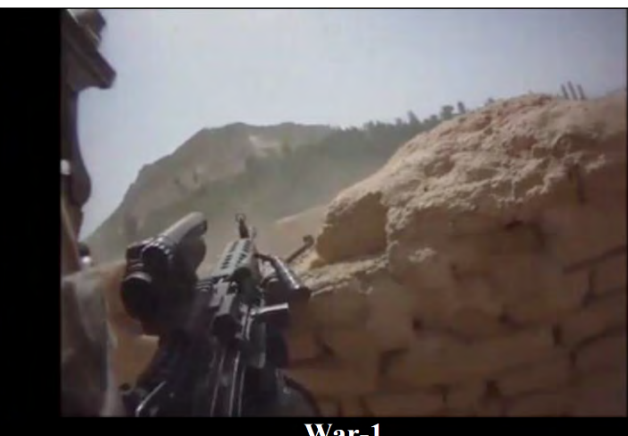

War-1

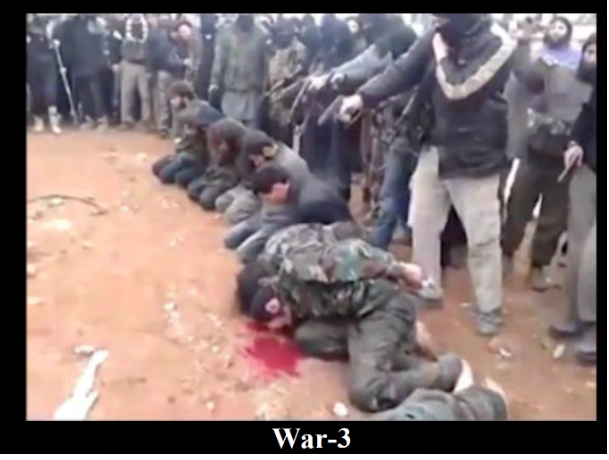

NEUR $\$$ SCIENCE

Figure 1. Sample screenshots of the presented videos

Sample screenshots of the videos being presented to the research participants during the fMRI scanning per three conditions; War-1: Real combat footage, without scenes relevant to murder, violence, or brutality; War-2: Real footage of combat with scenes relevant to murder and violence, but without brutality; War-3: Real videos of combat, including murder, violence, and brutality. Football: Videos relevant to a football game, presented as neutral stimulations. 
A checklist was followed by the examiner before each MRI scan to confirm the consent of the study subject and recognizing the study purposes. Additionally, no consumption of any antineuropathic medication, no consumption of any doping food (e.g. coffee, alcohol), or medicine on the exam day was checked. Moreover, the performance of clinical checks by the physician, setting lenses for better visualization of the goggle (if required), as well as goggle cleanness, training the participant for tasks during the scanning, requesting the participant to imaging being in the situation presented to him in the videos, and setting the headset volume on a comfortable level were observed.

The brain MRI was performed by a Siemens 3 Tesla MRI scanner (Prisma; Siemens Healthcare $\mathrm{GmbH}$, Federal Republic of Germany; Production: 2016) with a 64-channel head coil, at the National Brain Mapping Lab, Tehran City, Iran (www.nbml.ir). Functional T2×weighted images were collected using Blood Oxygen Level Dependent (BOLD) contrast, with $40 \mathrm{mT} / \mathrm{m}$ gradients, and by the coverage of the whole head. The protocol included a single-shot, spin-echo, Echoplanar Imaging $(\mathrm{EPI})$ sequence $(\mathrm{TR}=3000 \mathrm{~ms}, \mathrm{TE}=30 \mathrm{~ms}$, flip angle $=90^{\circ}, \mathrm{FOV}=192 \mathrm{~mm}^{2}$, matrix size $=64 \times 64, \&$ slice $\mathrm{gap}=0 \mathrm{~mm}$ ), and was used to acquire 40 slices with isotropic resolution $\left(3 \times 3 \times 3 \mathrm{~mm}^{3}\right)$. A Three-Dimensional (3D) T1-weighted anatomical scan was acquired prior to the EPI scan, using a gradient echo pulse sequence $(\mathrm{TR}=$ $1800 \mathrm{~ms}, \mathrm{TE}=3.44 \mathrm{~ms}, \mathrm{TI}=1100 \mathrm{~ms}$, flip angle $=7^{\circ}$, voxel size $=1 \times 1 \times 1 \mathrm{~mm}^{3}, \mathrm{FOV}=256 \times 256 \mathrm{~mm}^{2}$, matrix size $=$ $256 \times 256$, slice thickness $=1 \mathrm{~mm}, \&$ slice gap $=0 \mathrm{~mm}$ ). All obtained data were anonymized before processing.

Details of our volumetric analysis methods were previously published (Batouli, Sachdev, et al., 2014; Keihani et al., 2017). As a summary, initially, the quality of the T1-weighted scans was visually checked for a correct orientation and matrix size, for a proper signal to noise ratio, and no artifacts, using the Display toolbox, SPM12 (Welcome Department of Imaging Neuroscience: http:// www.fil.ion.ucl.ac.uk/spm), in MATLAB (version 9.0). No scan was excluded in this step. Next, VBM analysis was performed as follows. The scans were segmented into GM, WM, and CSF employing the segmentation toolbox, which created the native space plus Diffeomorphic Anatomical Registration Through Exponentiated Lie Algebra (DARTEL) imported outputs (Ashburner, 2010). This process was conducted using the default settings of the "Run DARTEL: create template" toolbox; the accuracy of inter-subject alignment was improved by iteratively averaging the DARTEL-imported data of the GM and WM tissue types to generate population- specific templates. Furthermore, after generating the templates, all the GM and WM images were normalized to the Montreal Neurological Institute (MNI) standard space, using the Normalize to MNI space toolbox. Eventually, all images were smoothed with a Gaussian kernel (10mm FWHM) before running the statistical analyses.

The total cerebral volume of all study subjects was estimated by adding the probability estimates of the GM and WM maps then multiplying the resulted values to $3.375 \mathrm{~mm}^{3}$ (the volume of one voxel). The total volumes were required for statistical inferences. Using global calculation, the study groups were compared in a voxel-wise manner, using Independent Samples t-test at the significance level of $\mathrm{P}<0.001$ (corrected for voxellevel multiple comparisons using the family-wise error correction). The implicit masking of the final images was eventually performed with a relative threshold. This measure helped to eliminate any effect of edge differences between the research groups.

The fMRI data analysis detailed was also previously published (Batouli, Hasani, Gheisari, Behzad, \& Oghabian, 2016). This analysis was performed using the fMRI Expert Analysis Tool (FEAT), part of FMRIB's Software Library (FSL, http://www.fmrib.ox.ac.uk/fsl) (version 5.0.9). Pre-processing steps included motion correction using FSL, Motion Correction from FMRIB's Linear Image Registration Tool (MCFLIRT); skullstripping for the removal of non-brain tissue using Brain Extraction Tool, FSL (BET); slice-timing correction by Fourier-space time-series phase-shifting; the normalization of the functional images to the standard Montreal Neurological Institute (MNI) brain atlas in two steps, as follows: I) the co-registration of each individual's functional images to his high-resolution T1-weighted scan, using FMRIB's Linear Image Registration (FLIRT) and 7 Degrees of Freedom (DOF); II) the linear registration of the structural T1 images to the MNI space, with 12 DOF; using a Gaussian kernel of FWHM= $6.0 \mathrm{~mm}$ for spatial smoothing; the multiplicative mean intensity normalization of the volume at each time point; and high pass temporal filtering (Gaussian-weighted least-squares straight-line fitting, with sigma $=60.0 \mathrm{~s}$ ).

The parametric statistical analysis was based on a General Linear Model (GLM) and performed using FEAT (version 6.0.0), FSL. The FMRIB Improved Linear Model (FILM) pre-whitening was implemented for the statistical analysis of the fMRI time-series. This procedure helped to make the statistical approaches valid and maximally efficient, which devoted a z-score to the corresponding BOLD signal. Three regressors were defined 
in the analysis, corresponding to the three war conditions. The individual GLM analyses were performed by creating a boxcar function of tasks (different conditions) against rest; being convolved with a canonical hemodynamic response function and its temporal derivatives. As explained above, we also registered the estimated function map to the corresponding structural image and ultimately the MNI space. For each subject, three contrast images were produced.

The group-level analysis was conducted applying FMRIB's Local Analysis of Mixed Effects (FLAME) to estimate within-group averages. Besides, it was employed for between-group comparisons using the three contrast images of the study subjects. Cluster thresholding was performed to only reveal the significantly-active clusters.
The initial criteria for identifying active clusters was a voxel-level probability threshold of $\mathrm{Z}>2.3$; a false discovery rate (PFDR $<0.05$ ) was also used to correct multiple comparisons; however, for a higher assurance of the findings, we finally selected a more stringent $\mathrm{P}<0.005$, which corresponded to a $Z>2.6$. The effect size of the comparisons between the two groups, based on Cohen's $\mathrm{d}$ formula (Kim, 2016) was estimated to be $\mathrm{d}=0.38$, i.e. regarded as a "medium" effect size (Kim, 2015). Furthermore, it is interpreted as $65 \%$ of the military group being above the mean value of the controls.

\section{Results}

The average brain activations of study groups in the three conditions were initially estimated. The details of these

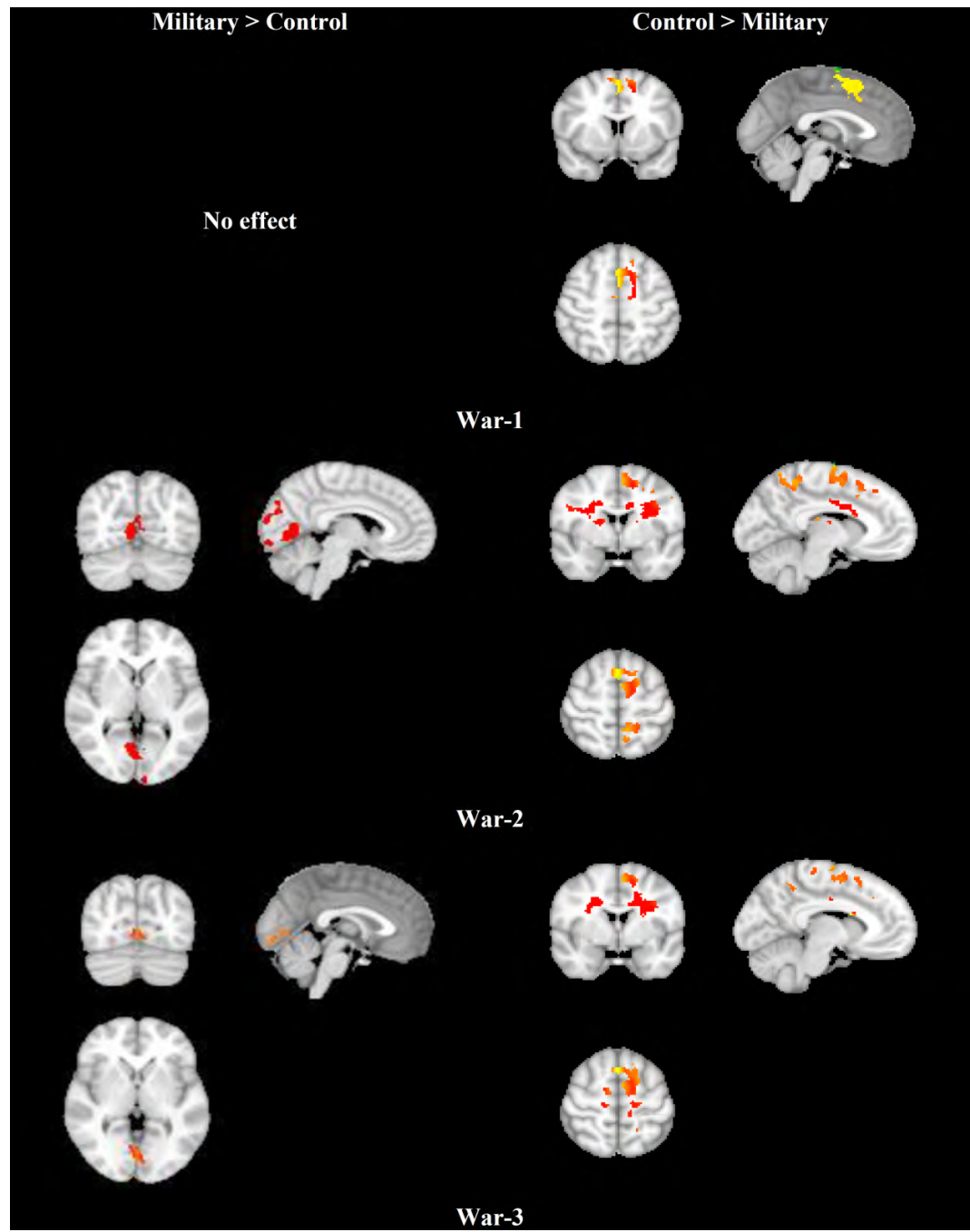

Figure 2. The fMRI data analysis results

The results of the contrasts between the fMRI maps of the military and control groups; the brain maps in the three war conditions were contrasted between the study groups to test any differences between their brain activations while observing the relevant videos; the military group presented higher activations in the occipital lobe of the brain, and this was mostly detected in the frontal areas for the control group. 
Table 1. Active brain areas during the three conditions

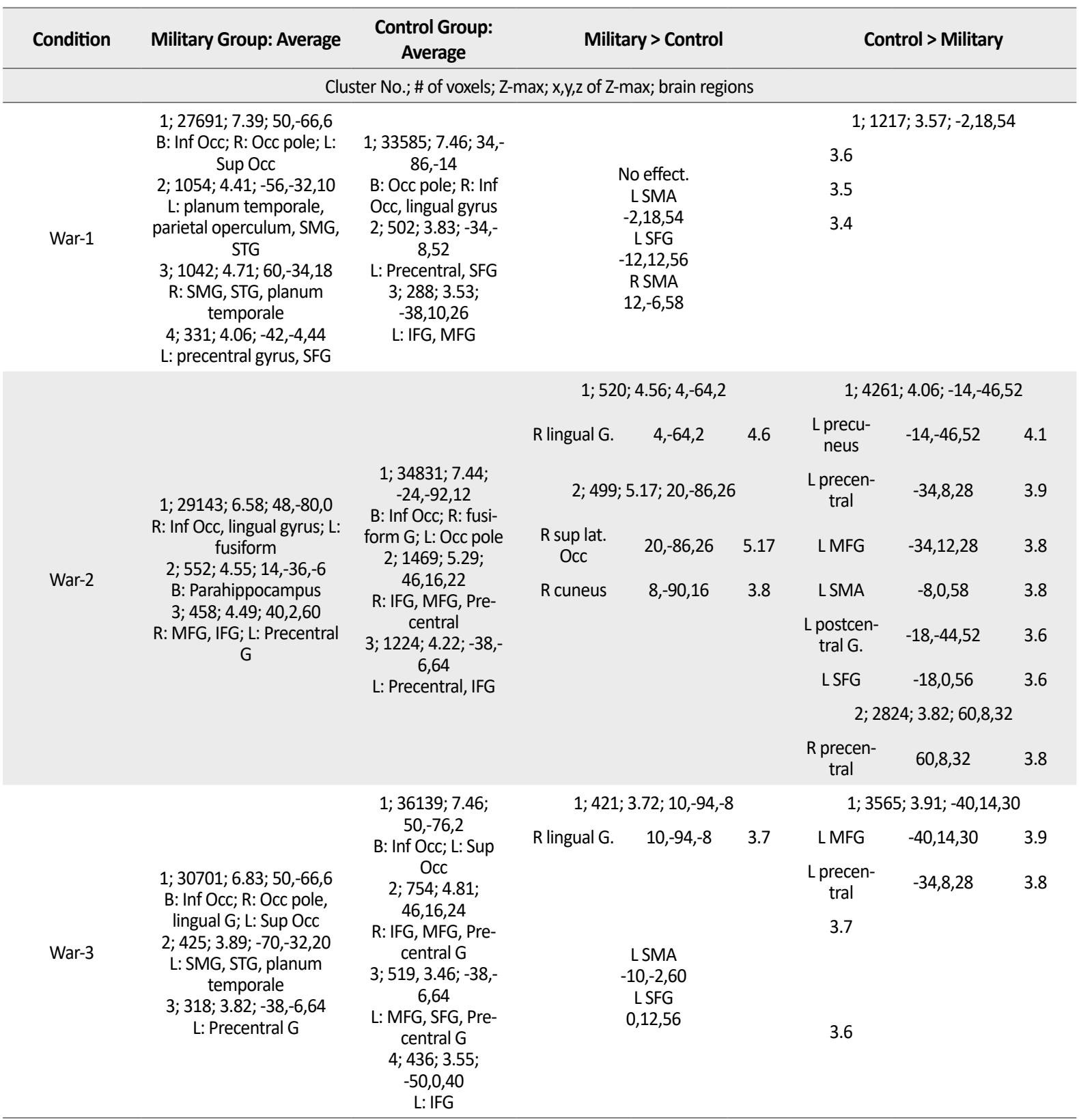

NEUR SCIENCE

Average brain activations of the military and control groups, as well as the contrasts between them, in addition to the number of voxels, z-value, coordinates, and included brain regions of each cluster; Maximum z-value and coordinates of the brain areas which showed different activations between the two groups are also provided; B: bilateral; R: right; L: left

activations, including size, coordinates, and the maximum $\mathrm{Z}$-value of the activation clusters are provided in Table 1.

Table 1 presents the average brain activations of the military and control groups, as well as the contrasts between them, in addition to the number of voxels, Zvalue, coordinates, and included brain regions of each cluster; maximum Z-value, and the coordinates of the brain areas, reflecting different activations between the research groups.

The first type of movies relevant to war activated bilateral Inf. Occ, planum temporale, Superior Temporal Gyrus (STG), and Supramarginal Gyrus (SMG) in the military group. These alternations were in addition to the right Occ pole, and the left Superior Occipital Gyrus (SOC), parietal operculum, Superior Frontal Gyrus 

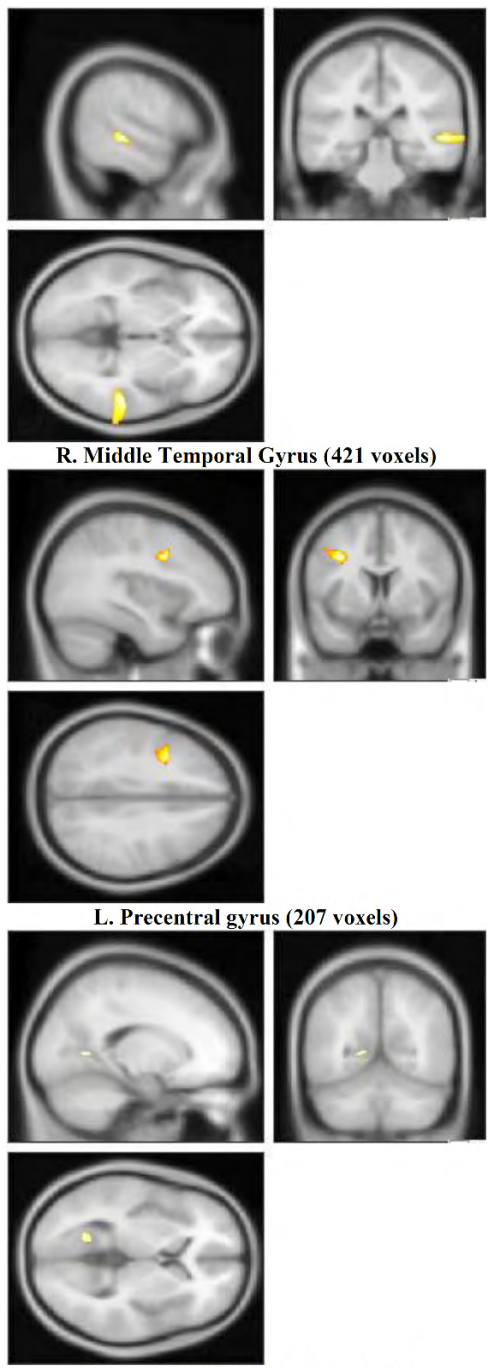

L. Posterior Cingulate (56 voxels)

Figure 3. The volumetric analysis data
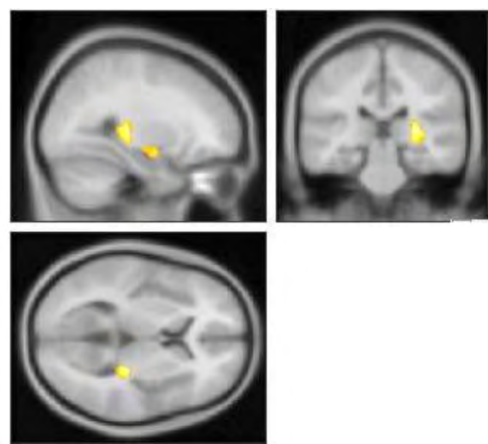

R. Hippocampus ( 288 voxels)
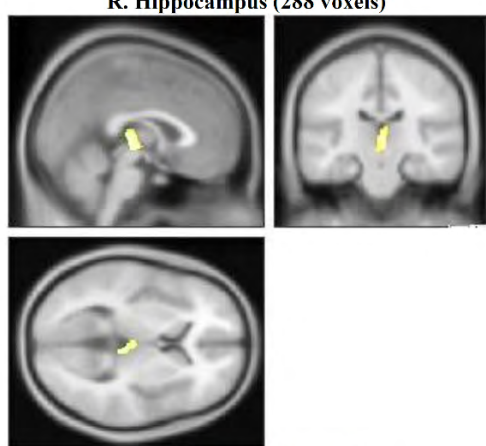

R. Thalamus (131 voxels)

The five areas of the brain which illustrated a larger volume in the military group, compared to controls, using a VBM analysis method; the areas included the right middle temporal gyrus, right hippocampus, left precentral gyrus, right thalamus, and left posterior cingulate. The colored pixels represent the brain regions overlaid on a standard (in the MNI space) T1-weighted scan of the brain.

(SFG), and precentral gyrus. The control group also presented brain activations in this condition in bilateral Occ pole, right Inf. Occ, lingual gyrus, left precentral, SFG, IFG, and MFG.

War-2 videos activated bilateral parahippocampus, right Inf. Occ, lingual gyrus, MFG, IFG, left fusiform, and precentral gyri in the military group. The brain activations of this condition in the control group included bilateral Inf. Occ, precentral, IFG, right fusiform, MFG, and left Occ pole.

The War-3 videos activated bilateral Inf Occ, right Occ pole, lingual gyrus, left SOG, SMG, STG, planum temporal, and precentral gyri in the military group. Further- more, the brain activations of the control group relevant to this condition were bilateral Inf Occ, IFG, MFG, precentral gyri, as well as the left SOG and SFG.

The brain activations of the study groups in the three conditions were contrasted during the analysis (military $>$ control \& control $>$ military); the relevant maps are provided in Figure 2. In the war-1 condition, the military group presented no higher brain activations, compared to the controls. However, bilateral SMA and left SFG were more active in the controls in this condition. During the war- 2 condition, the right lingual gyrus, superior lateral Occ, and cuneus were more active in the military group, compared to the controls. Besides, the bilateral precentral gyrus as well as the left precuneus, 
MFG, SMA, SFG, and postcentral gyrus were more active in the controls, compared to the military group. Finally, the right lingual gyrus was more active in the military group during the war-3 condition, whereas left MFG, SMA, SFG, and precentral gyri demonstrated higher activations in the controls.

The VBM analysis data revealed differences between the brain structures of the research groups, as illustrated in Figure 3. Accordingly, the military group presented larger volumes in 1103 voxels in 5 brain areas, including right middle temporal gyrus (421 voxels; x,y,z: 57,-31,2), right hippocampus (288 voxels; x,y,z: 28,-28,6), left precentral gyrus (207 voxels; $x, y, z:-36,6,36)$, right thalamus (131 voxels; x,y,z: 2,-22,4), and left posterior cingulate (56 voxels; x,y,z: $-17,-59,3)$. These results were obtained at a $\mathrm{P}<0.001,43$ DOFs, and $\mathrm{t}=3.29$.

\section{Discussion}

We compared structural and functional MRI scans of two groups of participants, including 20 professional military members and 26 healthy controls. Furthermore, the study groups were of the same age, educational level, socioeconomic status, and emotional state. Our results reported 1103 voxels in 5 brain areas to have a larger volume in the military group. In addition, the fMRI maps indicated differences between the brain activations of the two groups during observing movies relevant to stressful combat situations.

The patterns of brain activation varied between the study groups during the war conditions. Considering the three war conditions together, the military group suggested higher activations in the lingual and superior lateral occipital gyri, as well as the cuneus, all in the occipital lobe. In general, this brain lobe is involved in visual perception ( $\mathrm{Li}$ et al., 2015); perceiving the external stimuli; and transferring data to the brain regions related to emotion processing and responding (Adolphs, 2001). Additionally, these areas are involved in feature-extraction, shape recognition, and the integration of visual, phonological, and semantic information (Merriam \& Colby, 2005); visuomotor transformations necessary to orient in space, like juggling (Goodale \& Milner, 1992); processing an object's position (Gerber et al., 2014), or the speed of a moving stimulus (Rodman \& Albright, 1987). There are specific functional roles of the brain areas, i.e. more active in the military group, such as the occipitotemporal areas involved in feature extraction, shape recognition, as well as the integration of visual, semantic, and phonological information (Merriam \& Colby, 2005); or being active in tasks that stimulate visuospatial processing (Niemann, Godde, Staudinger, \& Voelcker-Rehage, 2014).
These data suggest that the military group allocated more resources for perceiving and processing the visual information, compared to the controls, i.e. consistent with previous research (Li et al., 2015).

On the other hand, the brain areas more active in the controls in this condition were Supplementary Motor Area (SMA), Primary Motor Cortex (PMC), superior and middle frontal gyri, and primary somatosensory, as well as the premotor cortices. These areas impact motor planning and execution (Doyon \& Benali, 2005). They also affect working memory, executive functions, and decision making (Jacini et al., 2009). The activation level of these areas also altered during stressful conditions (Ćosić et al., 2012). The suggestions for this pattern of activation in the controls could address their higher susceptibility towards stressful events, their lower inhibition capacity, and less experience of stressful situations. The specific roles of the active areas support this suggestion. For example, increased activation of precentral, postcentral, and middle frontal gyri is associated with negative emotional imaging (Li et al., 2015); the SMA is strongly associated with the primary motor, as well as the midcingulate cortices (Schlaffke et al., 2014). Therefore, it is involved in motor control (Dayan \& Cohen, 2011) and the planning of complex and coordinated movements (Chouinard \& Paus, 2006). Furthermore, this area influences coordinating the temporal sequences of actions, bimanual coordination, postural stability, and internally generated movements (Goble et al., 2010); the PMC affects response selection based on arbitrary cues, and the control of arms (Schlaffke et al., 2014), and sequential movements (Schubotz \& von Cramon, 2001). Besides, the ventral PMC is associated with mental imagination and movement observation (Caspers, Zilles, Laird, \& Eickhoff, 2010). The right PMC also reflects spatial processing, whereas the left is mainly involved in later phases of motor learning (Ghilardi et al., 2000).

We observed a lower functionality of the brain areas corresponding to motor actions and executive functions in the military group. One explanation could be their higher capacity for emotion regulation and response inhibition. This quality enabled them to modulate their brain activity. A study revealed that individuals who performed well under severe stress presented a more efficient functioning of the insula, i.e. a lower activation (Paulus et al., 2010). Another study indicated that a military group receiving a mindfulness training presented attenuated activations in the insula and ACC. These alternations occurred when observing emotional faces, the areas being involved in cognitive control, emotion regulation, reward monitoring, and interoception (Lutz 
et al., 2014). The extent of brain activation reduction in these areas was also associated with the level of resilience improvement after receiving mindfulness training. Reduced activation of ACC during a flanker task was also reported, being interpreted as a more efficient information processing in this region (Voelcker-Rehage, Godde, \& Staudinger, 2011). Furthermore, meditators reflected lower posterior insula activation during attentional control, compared to non-meditators (Brefczynski-Lewis, Lutz, Schaefer, Levinson, \& Davidson, 2007). One other explanation for lower brain activation in the military group could be the forebrain-to-midbrain switch when the threat is proximal, and the role of vmPFC in fear inhibition (Mobbs et al., 2009). The default network of the brain becomes deactivated when a critical external cue is presented (Greicius, Krasnow, Reiss, \& Menon, 2003). Besides, this deactivation, like the one in the PFC, is associated with devoting external attention to the environment (Buckner, Andrews-Hanna, \& Schacter, 2008) to more specifically react to a potential threat. These findings suggest that specific types of mental training are correlated with the decreased requirement of cognitive resources to perform a task ( $\mathrm{Ph}$ et al., 2015); they also help in more efficient processing of stress cues.

The biopsychological training also assisted soldiers with their recovery from stress after the deployment $(\mathrm{Ph}$ et al., 2015). Furthermore, practicing emotion regulation methods helps individuals to replace ineffective decision-making with a productive response to stressful and challenging conditions (Oden et al., 2015). This result is important, as the military relies on preparing warfighters for highly-complex situations.

Contrarily, we observed a higher activation in the military group during the war condition in the brain areas relevant to visual processing. There is substantial evidence that coordination training leads to increased activation of the visuospatial network (Voelcker-Rehage et al., 2011). In general, physical training could provide functional changes in specific brain areas, leading to a general improvement in human cognition (Duchesne et al., 2016). Scholars documented alternations in the electrical oscillations of the brain due to physical training (Ermutlu, Yücesir, Eskikurt, Temel, \& İşoğlu-Alkaç, 2015). Twelve months of resistance training demonstrated functional changes in two brain areas associated with response inhibition, middle temporal gyrus, and anterior insula extending into lateral OFC (Liu-Ambrose et al., 2012); even one week of training-induced learning-related changes in the functional connectivity of brain areas was reported (Della-Maggiore \& McIntosh, 2005). One suggested mechanism for the association between PA and the brain func- tion alteration is that chronic exposure to cardiovascular and resistance training is correlated with increased metabolic demands. Therefore, the need for glucose and oxygen increases in the active neurons (Ding et al., 2004). Besides, a lower BMI is associated with a healthier brain and an improved cognitive capacity (Flöel et al., 2010).

The brain volume was larger in 5 areas in the military group, compared to the controls. The human brain is elastic, i.e. its structure alters to adapt to new demands (Lövdén, Bäckman, Lindenberger, Schaefer, \& Schmiedek, 2010 ). Our previous review study suggested that $>80 \%$ of the GM of the brain was positive associated with PA (Batouli \& Saba, 2017). Moreover, all the 5 brain regions in that review study presented such association; the number of studies (among 53 studies) that reported these 5 regions to correlated with PA was 14 for the hippocampus, 9 for the middle temporal gyrus, 4 for the precentral gyrus and posterior cingulate, and 1 for the thalamus.

It is reported that those areas of the brain which present structural changes in association with PA are mainly essential for motor-related functions (Lerch et al., 2011; Mårtensson et al., 2012). For instance, the hippocampus presents increased neurogenesis and a higher cerebral blood volume induced by exercise (Pereira et al., 2007). It is also responsible for the encoding and consolidation of motor skills (Albouy et al., 2008); the middle temporal gyrus is significantly involved in motor learning and memory (Doyon \& Benali, 2005); the precentral gyrus is a primary site of the motor cortex; cingulate cortex coordinates complex motor movements concerning higher-order attentional demands (Rovio et al., 2010); PCC mediates executive functions (Hillman, Erickson, \& Kramer, 2008), and in particular, integrates multimodal sensory data for the planning and execution of complex movements (Amiez \& Petrides, 2014); thalamus relays sensory-motor signals to the cerebral cortex and is known as a subcortical motor center. Despite involvement in motor-related functions, these areas are engaged in other cognitive abilities as well. These data explain the association of PA with general cognitive improvements among individuals. For example, the hippocampus impacts navigation and spatial representation skills (Stickgold, Malia, Maguire, Roddenberry, \& O'Connor, 2000), spatial memory (Lee, Miyasato, \& Clayton, 1998), and retrieval (Brodziak, 2013); PCC is associated with navigation ability (Maguire et al., 2000), spatial memory (Friedman et al., 1998), the recognition and retrieval of previously encountered information (Liégeois et al., 2004), semantic priming (O’Hare, Dien, Waterson, \& Savage, 2008), and moral judgments (Raine \& Yang, 
2006); and precentral gyrus is involved in spatial working memory (Weinstein et al., 2012).

There are some candidate mechanisms for the activity-related structural changes of the brain. PA results in elevated blood flow and volume in the brain (Yau, GilMohapel, Christie, \& So, 2014). PA is also related to the growth of capillaries (Palmer, Willhoite, \& Gage, 2000) and the number of small blood vessels (Bullitt et al., 2009). Considering that 5\% of GM tissue is accounted for vasculature (Barbier, Lamalle, \& Décorps, 2001), any elevation of the above-mentioned factors could contribute to the increment of the neural volume. In addition, the increment of blood flow in the brain could be associated with the development of new neurons (Dishman et al., 2006; Draganski \& May, 2008; Pereira et al., 2007), i.e. interpreted as a larger GM volume or density. A few candidate mechanisms include elevated cell proliferation (Maguire et al., 2003), angiogenesis (Cotman \& Berchtold, 2002), neurogenesis (Heo et al., 2014; van Praag, Kempermann, \& Gage, 2000), synaptogenesis (Kleim et al., 2002; Kobilo et al., 2011), and myelination (Canu, Carnaud, Picquet, \& Goutebroze, 2009; Ruegg, Kakebeeke, Gabriel, \& Bennefeld, 2003). Hippocampus is among the brain regions where neurogenesis mostly occurs (Gross, 2000). One explanation could be that PA elevates the level of some proteins in the brain, such as High-Density Lipoprotein-Cholesterol (HDL-C); Insulin-Like Growth Factor 1 (IGF-1); Vascular Endothelial Growth Factor (VEGF) (Cassilhas et al., 2007); the level of Brain-Derived Neurotrophic Factor (BDNF) (Tang, Chu, Hui, Helmeste, \& Law, 2008; Voss, Vivar, Kramer, \& van Praag, 2013), and the Nerve Growth Factor (NGF) (Ang, 2007). Besides, these proteins and factors support the above-mentioned processes, such as the neural growth and survival (Cao et al., 2007), cell genesis (van Praag, 2009), and the formation of new blood vessels (Hillman et al., 2008). Finally, PA alters the concentration of brain metabolites, such as glutamate/glutamine (GLX) (Biedermann et al., 2012), NAA (Erickson et al., 2012; Gonzales et al., 2013), and lactate (Kuipers, Keizer, Vries, Rijthoven, \& Wijts, 1988). It has been documented that one explanation for BV changes could be alterations in the level of brain metabolites (Pajonk \& et al., 2010).

The explored military group was trained with the basic physical exercises of the army for approximately three years. Studies have illustrated that different types, durations, and volumes of PAs present various levels of association with the brain structure. Different types of PAs are associated with the brain structure; from aerobic exercise, endurance, and balance training, to sports, such as biking, martial arts, and ball sports, as well as to general physical activities, such as dancing, gardening, and walking (Batouli \& Saba, 2017), albeit with different levels of association. For example, a larger hippocampal volume was observed in endurance athletes, compared to martial artists (Schlaffke et al., 2014). The duration of exercise is another determining factor in this respect. The time-scale for the structural changes of the brain in association with PA is much shorter than 10 years of practicing Judo (Jacini et al., 2009) or 5 years of dancing (Niemann et al., 2016); 6 weeks of aerobic exercise (Thomas et al., 2016), 1 week of practicing a complex visuomotor rotation task (Landi, Baguear, \& Della-Maggiore, 2011), or even 4 days of practicing the signature with the non-dominant hand (Hamzei, Glauche, Schwarzwald, \& May, 2012). The PA volume is also critical, and the reports range from 6.5 hours (Wei, Zhang, Jiang, \& Luo, 2011) or 45 minutes (Sehm et al., 2014) per day, to 180 (Colcombe et al., 2006; Niemann et al., 2014) or 120 minutes (Mueller et al., 2015) per week. It is suggested that short-term and high-volume, as well as long-term and low-volume PAs, are positively associated with the brain structure (Ruscheweyh et al., 2011).

This study aimed at identifying the associations between military training and the structural and functional changes in the brain. We considered strict inclusion criteria for the study participants, and selected robust imaging and analysis methods, to obtain reliable results. We presented real combat movies to the research participants to simulate the situation for them. Subsequently, it was revealed that brain areas illustrated higher activity in response to dynamic versus static emotional stimuli (Trautmann, Fehr, \& Herrmann, 2009). Despite its strengths, this study had a few limitations. Although hurting humans is morally prohibited, on some occasions, such as warfare, killing enemy soldiers becomes justified (Molenberghs et al., 2015). Besides, the neural circuits of justified and unjustified killings may be different, i.e. not tested here. Second, the optimal approach to examine the association of PA with $\mathrm{BV}$ is comparing volumetric measures before and after a period of exercise, i.e. impossible in the present research. Additionally, although we tried to match the participants of the two groups for age, handedness, gender, educational level, socioeconomic status, and the emotional state, the possibility of inter-individual differences and their influences on our findings could not be neglected. Eventually, a PANAS test after the imaging session, to assess the behavioral responses of the participants towards the movies, could have added useful data to our findings. 


\section{Conclusion}

Our results were different with the only similar study identified. A study (Ćosić et al., 2012) presented that the military group had higher activations in the posterior parietal, posterior temporal, premotor, and prefrontal areas of the brain while watching the videos of combat, compared to the controls. The number of active ROIs, as well as the overall number of active voxels, were also higher in their military group. They interpreted their findings as the military group to have a better understanding of the conditions of the videos. We agree with their interpretation; however, this was a pilot study with only two combat servicemen versus two mission-ready soldiers. Thus, replication might be required.

Furthermore, several studies have reported negative associations of military deployments with brain health and cognitive abilities. Our military group was under training and had never experienced a real combat situation; therefore, our results on the positive associations of military training with the alteration of brain structure and function could not be in contrast with previous investigations.

Besides the applications in the military, our findings are beneficial for providing healthy aging. Brain volume declines in the elderly (Batouli et al., 2014; Raz et al., 2004; Sachdev et al., 2013), and the human brain is under the influences of both genetic and environmental factors (Sachdev et al., 2013). Accordingly, the heritability of brain volume decreases in old age, and the impact of environmental factors increases (Batouli et al., 2014; Batouli, Trollor, Wen, \& Sachdev, 2014). Thus, providing beneficial environmental factors and a healthy lifestyle for the elderly seems to be the best approach for healthy aging (Batouli et al., 2014). A study indicated that only one year of aerobic exercise was equal to adding 1 to 2 years to brain health (Erickson et al., 2010).

\section{Ethical Considerations}

\section{Compliance with ethical guidelines}

This study was approved by the Ethics Committee of Iran University of Medical Sciences (Code: IR.IUMS. REC.1395.899).

\section{Funding}

AJA University of Medical Sciences financially supported this work.
Authors' contributions

All authors equally contributed in preparing this article.

\section{Conflict of interest}

The authors declared no conflict of interest.

Acknowledgments

We thank the cooperation of the National Brain Mapping Lab (NBML) in this study.

\section{References}

Adolphs, R. (2001). The neurobiology of social cognition. Current Opinion in Neurobiology, 11(2), 231-9. [DOI:10.1016/S09594388(00)00202-6]

Albouy, G., Sterpenich, V., Balteau, E., Vandewalle, G., Desseilles, M., \& Dang-Vu, T., et al. (2008). Both the hippocampus and striatum are involved in consolidation of motor sequence memory. Neuron, 58(2), 261-72. [DOI:10.1016/j.neuron.2008.02.008] [PMID]

Amiez, C., \& Petrides, M. (2014). Neuroimaging evidence of the anatomo-functional organization of the human cingulate motor areas. Cerebral Cortex, 24(3), 563-78. [DOI:10.1093/cercor/ bhs329] [PMID]

Ang, E. T., \& Gomez-Pinilla, F. (2007). Potential therapeutic effects of exercise to the brain. Current Medicinal Chemistry, 14(24), 2564-71. [DOI:10.2174/092986707782023280] [PMID]

Ashburner, J. (2010). VBM tutorial. Retrieved from https:// www.fil.ion.ucl.ac.uk/ john/misc/VBMclass10.pdf

Asmundson, G. J. G., Fetzner, M. G., DeBoer, L. B., Powers, M B., Otto, M. W., \& Smits, J. A. J. (2013). Let's get physical: A contemporary review of the anxiolytic effects of exercise for anxiety and its disorders. Depression and Anxiety, 30(4), 362-73. [DOI:10.1002/da.22043] [PMID]

Barbier, E. L., Lamalle, L., \& Décorps, M. (2001). Methodology of brain perfusion imaging. Journal of Magnetic Resonance Imaging, 13(4), 496-520. [DOI:10.1002/jmri.1073] [PMID]

Barnes, D. E., Yaffe, K., Satariano, W. A., \& Tager, I. B. (2003). A longitudinal study of cardiorespiratory fitness and cognitive function in healthy older adults. Journal of the American Geriatrics Society, 51(4), 459-65. [DOI:10.1046/j.15325415.2003.51153.x] [PMID]

Batouli, S. A. H., Hasani, N., Gheisari, S., Behzad, E., \& Oghabian, M. A. (2016). Evaluation of the factors influencing brain language laterality in presurgical planning. Physica Medica, 32(10), 1201-9. [DOI:10.1016/j.ejmp.2016.06.008] [PMID]

Batouli, S. A. H., \& Saba, V. (2017). At least eighty percent of brain grey matter is modifiable by physical activity: A review study. Behavioural Brain Research, 332(Supplement C), 204-17. [DOI:10.1016/j.bbr.2017.06.002] [PMID] 
Batouli, S. A. H., Sachdev, P. S., Wen, W., Wright, M. J., Ames, D., \& Trollor, J. N. (2014). Heritability of brain volumes in older adults: The older Australian twins study. Neurobiology of Aging, 35(4), 937.e5-18. [DOI:10.1016/j.neurobiolaging.2013.10.079] [PMID]

Batouli, S. A. H., \& Sisakhti, M (2019). Investigating A Hypothesis on The Mechanism of Long-term Memory Storage. NeuroQuantology, 17(3) . [DOI:10.14704/NQ.2019.17.3.1813] https://www.neuroquantology.com/index.php/journal/ article/view/1813/1346

Batouli, S. A. H., Trollor, J. N., Wen, W., \& Sachdev, P. S. (2014) The heritability of volumes of brain structures and its relationship to age: A review of twin and family studies. Ageing Research Reviews, 13, 1-9. [DOI:10.1016/j.arr.2013.10.003] [PMID]

Biedermann, S., Fuss, J., Zheng, L., Sartorius, A., Falfán-Melgoza, C., \& Demirakca, T., et al. (2012). In vivo voxel based morphometry: Detection of increased hippocampal volume and decreased glutamate levels in exercising mice. NeuroImage, 61(4), 1206-12. [DOI:10.1016/j.neuroimage.2012.04.010] [PMID]

Braskie, M. N., Boyle, C. P., Rajagopalan, P., Gutman, B. A., Toga, A. W., \& Raji, C. A., et al. (2014). Physical activity, inflammation, and volume of the aging brain. Neuroscience, 273 , 199-209. [DOI:10.1016/j.neuroscience.2014.05.005] [PMID] [PMCID]

Brefczynski-Lewis, J. A., Lutz, A., Schaefer, H. S., Levinson, D. B., \& Davidson, R. J. (2007). Neural correlates of attentional expertise in long-term meditation practitioners. Proceedings of the National Academy of Sciences of the United States of America, 104(27), 11483-8. [DOI:10.1073/pnas.0606552104] [PMID] [PMCID]

Brodziak, A. (2013). A current model of neural circuitry active in forming mental images. Medical Science Monitor: International Medical Journal of Experimental and Clinical Research, 19, 1146-58. [DOI:10.12659/MSM.889587] [PMID] [PMCID]

Bullitt, E., Rahman, F. N., Smith, J. K., Kim, E., Zeng, D., \& Katz, L. M., et al. (2009). The effect of exercise on the cerebral vasculature of healthy aged subjects as visualized by MR angiography. American Journal of Neuroradiology, 30(10), 1857 LP-1863. [DOI:10.3174/ajnr.A1695] [PMID] [PMCID]

Canu, M. H., Carnaud, M., Picquet, F., \& Goutebroze, L. (2009). Activity-dependent regulation of myelin maintenance in the adult rat. Brain Research, 1252, 45-51. [DOI:10.1016/j.brainres.2008.10.079] [PMID]

Cao, L., Dhilla, A., Mukai, J., Blazeski, R., Lodovichi, C., \& Mason, C. A., et al. (2007). Genetic modulation of BDNF signaling affects the outcome of axonal competition in vivo. Current Biology, 17(11), 911-21. [DOI:10.1016/j.cub.2007.04.040] [PMID] [PMCID]

Caspers, S., Zilles, K., Laird, A. R., \& Eickhoff, S. B. (2010). ALE meta-analysis of action observation and imitation in the human brain. NeuroImage, 50(3), 1148-67. [DOI:10.1016/j.neuroimage.2009.12.112] [PMID] [PMCID]

Cassilhas, R. C., \& Viana, V. A. R., Grassmann, V., Santos, R. T., Santos, R. F., \& Tufik, S., et al. (2007). The impact of resistance exercise on the cognitive function of the elderly. Medicine E Science in Sports \& Exercise, 39(8), 1401-7. [DOI:10.1249/ mss.0b013e318060111f] [PMID]
Chouinard, P. A., \& Paus, T. (2006). The primary motor and premotor areas of the human cerebral cortex. The Neuroscientist, 12(2), 143-52. [DOI:10.1177/1073858405284255] [PMID]

Chrousos, G. P. (2009). Stress and disorders of the stress system. Nature Reviews Endocrinology, 5(7), 374-81. [DOI:10.1038/ nrendo.2009.106] [PMID]

Colcombe, S., Erickson, K., Scalf, P., Kim, J., Prakash, R., \& McAuley, E., et al. (2006). Aerobic exercise training increases brain volume in aging humans. The Journals of Gerontology: Series A, Biological Sciences and Medical Sciences, 61(11), 1166-70. [DOI:10.1093/gerona/61.11.1166] [PMID]

Colcombe, S., \& Kramer, A. F. (2003). Fitness effects on the cognitive function of older adults. Psychological Science, 14(2), 125-30 [DOI:10.1111/1467-9280.t01-1-01430] [PMID]

Ćosić, K., Popović, S., Fabek, I., Kovač, B., Radoš, M., \& Radoš, M., et al. (2012). fMRI neural activation patterns induced by professional military training. Translational Neuroscience, 3(1), 46-50. [DOI:10.2478/s13380-012-0012-2]

Cotman, C. W., \& Berchtold, N. C. (2002). Exercise: A behavioral intervention to enhance brain health and plasticity. Trends in Neurosciences, 25(6), 295-301. [DOI:10.1016/S01662236(02)02143-4]

Dayan, E., \& Cohen, L. G. (2011). Neuroplasticity subserving motor skill learning. Neuron, 72(3), 443-54. [DOI:10.1016/j neuron.2011.10.008] [PMID] [PMCID]

Della-Maggiore, V., \& McIntosh, A. R. (2005). Time course of changes in brain activity and functional connectivity associated with long-term adaptation to a rotational transformation. Journal of Neurophysiology, 93(4), 2254-62. [DOI:10.1152/ jn.00984.2004] [PMID]

Ding, Y., Li, J., Luan, X., Ding, Y. H., Lai, Q., \& Rafols, J. A., et al. (2004). Exercise pre-conditioning reduces brain damage in ischemic rats that may be associated with regional angiogenesis and cellular overexpression of neurotrophin. Neuroscience, 124(3), 583-91. [DOI:10.1016/j.neuroscience.2003.12.029] [PMID]

Dishman, R. K., Berthoud, H. R., Booth, F. W., Cotman, C. W., Edgerton, V. R., \& Fleshner, M. R., et al. (2006). Neurobiology of Exercise. Obesity, 14(3), 345-56. [DOI:10.1038/oby.2006.46] [PMID]

Doyon, J., \& Benali, H. (2005). Reorganization and plasticity in the adult brain during learning of motor skills. Current Opinion in Neurobiology, 15(2), 161-7. [DOI:10.1016/j.conb.2005.03.004] [PMID]

Draganski, B., \& May, A. (2008). Training-induced structural changes in the adult human brain. Behavioural Brain Research, 192(1), 137-42. [DOI:10.1016/j.bbr.2008.02.015] [PMID]

Duchesne, C., Gheysen, F., Bore, A., Albouy, G., Nadeau, A., \& Robillard, M. E., et al. (2016). Influence of aerobic exercise training on the neural correlates of motor learning in Parkinson's disease individuals. NeuroImage: Clinical, 12, 559-69. [DOI:10.1016/j.nicl.2016.09.011] [PMID] [PMCID]

Eiland, L., \& Romeo, R. D. (2013). Stress and the developing adolescent brain. Neuroscience, 249(Supplement C), 162-71. [DOI:10.1016/j.neuroscience.2012.10.048] [PMID] [PMCID]

Erickson, K. I., Raji, C. A., Lopez, O. L., Becker, J. T., Rosano, C., \& Newman, A. B., et al. (2010). Physical activity predicts 
gray matter volume in late adulthood: The cardiovascular health study(e-pub ahead of print). Neurology, 75(16), 1415-22. [DOI:10.1212/WNL.0b013e3181f88359] [PMID] [PMCID]

Erickson, K. I., Weinstein, A. M., Sutton, B. P., Prakash, R. S., Voss, M. W., \& Chaddock, L., et al. (2012). Beyond vascularization: Aerobic fitness is associated with N-acetylaspartate and working memory. Brain and Behavior, 2(1), 32-41. [DOI:10.1002/brb3.30] [PMID] [PMCID]

Ermutlu, N., Yücesir, I., Eskikurt, G., Temel, T., \& İşoğlu-Alkaç, Ü. (2015). Brain electrical activities of dancers and fast ball sports athletes are different. Cognitive Neurodynamics, 9(2), 257-63. [DOI:10.1007/s11571-014-9320-2] [PMID] [PMCID]

Flöel, A., Ruscheweyh, R., Krüger, K., Willemer, C., Winter, B., \& Völker, K., et al. (2010). Physical activity and memory functions: Are neurotrophins and cerebral gray matter volume the missing link? NeuroImage, 49(3), 2756-63. [DOI:10.1016/j.neuroimage.2009.10.043] [PMID]

Friedman, L., Kenny, J. T., Wise, A. L., Wu, D., Stuve, T. A., \& Miller, D. A., et al. (1998). Brain activation during silent word generation evaluated with functional MRI. Brain and Language, 64(2), 231-56. [DOI:10.1006/brln.1998.1953] [PMID]

Gerber, P., Schlaffke, L., Heba, S., Greenlee, M. W., Schultz, T., \& Schmidt-Wilcke, T. (2014). Juggling revisited - A voxel-based morphometry study with expert jugglers. NeuroImage, 95, 320-5. [DOI:10.1016/j.neuroimage.2014.04.023] [PMID]

Ghilardi, M.-F., Ghez, C., Dhawan, V., Moeller, J., Mentis, M., \& Nakamura, T., et al. (2000). Patterns of regional brain activation associated with different forms of motor learning. Brain Research, 871(1), 127-45. [DOI:10.1016/S0006-8993(00)02365-9]

Goble, D. J., Coxon, J. P., Van Impe, A., De Vos, J., Wenderoth, N., \& Swinnen, S. P. (2010). The neural control of bimanual movements in the elderly: Brain regions exhibiting age-related increases in activity, frequency-induced neural modulation, and task-specific compensatory recruitment. Human Brain Mapping, 31(8), 1281-95. [DOI:10.1002/hbm.20943] [PMID] [PMCID]

Gonzales, M. M., Tarumi, T., Kaur, S., Nualnim, N., Fallow, B. A., \& Pyron, M., et al. (2013). Aerobic fitness and the brain: Increased $\mathrm{N}$-Acetyl-Aspartate and choline concentrations in endurancetrained middle-aged adults. Brain Topography, 26(1), 126-34. [DOI:10.1007/s10548-012-0248-8] [PMID] [PMCID]

Goodale, M. A., \& Milner, A. D. (1992). Separate visual pathways for perception and action. Trends in Neurosciences, 15(1), 20-5. [DOI:10.1016/0166-2236(92)90344-8]

Greicius, M. D., Krasnow, B., Reiss, A. L., \& Menon, V. (2003). Functional connectivity in the resting brain: A network analysis of the default mode hypothesis. Proceedings of the National Academy of Sciences of the United States of America, 100(1), 253-8. [DOI:10.1073/pnas.0135058100] [PMID] [PMCID]

Gross, C. G. (2000). Neurogenesis in the adult brain: Death of a dogma. Nature Reviews Neuroscience, 1(1), 67-73. [DOI:10.1038/35036235] [PMID]

Hamzei, F., Glauche, V., Schwarzwald, R., \& May, A. (2012). Dynamic gray matter changes within cortex and striatum after short motor skill training are associated with their increased functional interaction. NeuroImage, 59(4), 3364-72. [DOI:10.1016/j.neuroimage.2011.10.089] [PMID]
Hellhammer, D. H., Wüst, S., \& Kudielka, B. M. (2009). Salivary cortisol as a biomarker in stress research. Psychoneuroendocrinology, 34(2), 163-71. [DOI:10.1016/j.psyneuen.2008.10.026] [PMID]

Heo, Y. M., Shin, M.S., Kim, S. H., Kim, T. W., Baek, S. B., \& Baek, S. S. (2014). Treadmill exercise ameliorates disturbance of spatial learning ability in scopolamine-induced amnesia rats. Journal of Exercise Rehabilitation, 10(3), 155-61. [DOI:10.12965/ jer.140110] [PMCID]

Herrera, J. J., Fedynska, S., Ghasem, P. R., Wieman, T., Clark, P. J., \& Gray, N., et al. (2016). Neurochemical and behavioral indices of exercise reward are independent of exercise controllability. The European Journal of Neuroscience, 43(9), 1190-202. [DOI:10.1111/ejn.13193] [PMID] [PMCID]

Hillman, C. H., Erickson, K. I., \& Kramer, A. F. (2008). Be smart, exercise your heart: Exercise effects on brain and cognition. Nature Reviews Neuroscience, 9(1), 58-65. [DOI:10.1038/ nrn2298] [PMID]

Jacini, W. F. S., Cannonieri, G. C., Fernandes, P. T., Bonilha, L., Cendes, F., \& Li, L. M. (2009). Can exercise shape your brain? Cortical differences associated with judo practice. Journal of Science and Medicine in Sport, 12(6), 688-90. [DOI:10.1016/j. jsams.2008.11.004] [PMID]

Keihani, A., Ekhtiari, H., Batouli, S. A. H., Shahbabaie, A., Sadighi, N., \& Mirmohammad, M., et al. (2017). Lower gray matter density in the anterior cingulate cortex and putamen can be traceable in chronic heroin dependents after over three months of successful abstinence. Iranian Journal of Radiology, 14(3), e41858. [DOI:10.5812/iranjradiol.41858]

Kim, H. Y. (2015). Statistical notes for clinical researchers: Effect size. Restorative Dentistry \& Endodontics, 40(4), 328-31. [DOI:10.5395/rde.2015.40.4.328] [PMID] [PMCID]

Kim, H. Y. (2016). Statistical notes for clinical researchers: Sample size calculation 1. comparison of two independent sample means. Restorative Dentistry \& Endodontics, 41(1), 74-8. [DOI:10.5395/ rde.2016.41.1.74] [PMID] [PMCID]

Kleim, J. A., Barbay, S., Cooper, N. R., Hogg, T. M., Reidel, C. N., \& Remple, M. S., et al. (2002). Motor Learning-dependent synaptogenesis is localized to functionally reorganized motor cortex. Neurobiology of Learning and Memory, 77(1), 63-77. [DOI:10.1006/ nlme.2000.4004] [PMID]

Kobilo, T., Liu, Q. R., Gandhi, K., Mughal, M., Shaham, Y., \& van Praag, H. (2011). Running is the neurogenic and neurotrophic stimulus in environmental enrichment. Learning $\mathcal{E}$ Memory, 18(9), 605-9. [DOI:10.1101/lm.2283011] [PMID] [PMCID]

Kramer, A. F., Hahn, S., Cohen, N. J., Banich, M. T., McAuley, E., \& Harrison, C. R., et al. (1999). Ageing, fitness and neurocognitive function. Nature, 400(6743), 418-9. [DOI:10.1038/22682] [PMID]

Kuipers, H., Keizer, H. A., De Vries, T., Van Rijthoven, P., \& Wijts, M. (1988). Comparison of heart rate as a non-invasive determinant of anaerobic threshold with the lactate threshold when cycling. European Journal of Applied Physiology and Occupational Physiology, 58(3), 303-6. [DOI:10.1007/BF00417267] [PMID]

Landi, S. M., Baguear, F., \& Della-Maggiore, V. (2011). One week of motor adaptation induces structural changes in primary motor cortex that predict long-term memory one year later. The Journal of Neuroscience: The Official Journal of the Society for Neuroscience, 31(33), 11808-13. [DOI:10.1523/JNEUROSCI.2253-11.2011] [PMID] [PMCID] 
Lee, D. W., Miyasato, L. E., \& Clayton, N. S. (1998). Neurobiological bases of spatial learning in the natural environment: Neurogenesis and growth in the avian and mammalian hippocampus. Neuroreport, 9(7), 15-27. [DOI:10.1097/00001756-199805110-00076]

Lerch, J. P., Yiu, A. P., Martinez-Canabal, A., Pekar, T., Bohbot, V. D., \& Frankland, P. W., et al. (2011). Maze training in mice induces MRI-detectable brain shape changes specific to the type of learning. NeuroImage, 54(3), 2086-95. [DOI:10.1016/j.neuroimage.2010.09.086] [PMID]

Lerner, J., \& Keltner, D. (2001). Fear, anger, and risk. Journal of Personality and Social Psychology, 81(1), 146-59. [DOI:10.1037/00223514.81.1.146] [PMID]

Li, G., Ma, X., Bian, H., Sun, X., Zhai, N., \& Yao, M., et al. (2015). A pilot fMRI study of the effect of stressful factors on the onset of depression in female patients. Brain Imaging and Behavior, 10(1), 195-202. [DOI:10.1007/s11682-015-9382-8] [PMID] [PMCID]

Liégeois, F., Connelly, A., Cross, J. H., Boyd, S. G., Gadian, D. G., \& Vargha-Khadem, F., et al. (2004). Language reorganization in children with early-onset lesions of the left hemisphere: An fMRI study. Brain, 127(6), 1229-36. [DOI:10.1093/brain/awh159] [PMID]

Liu-Ambrose, T., Nagamatsu, L. S., Voss, M. W., Khan, K. M., \& Handy, T. C. (2012). Resistance training and functional plasticity of the aging brain: A 12-month randomized controlled trial. Neurobiology of Aging, 33(8), 1690-8. [DOI:10.1016/j.neurobiolaging.2011.05.010] [PMID]

Lövdén, M., Bäckman, L., Lindenberger, U., Schaefer, S., \& Schmiedek, F. (2010). A theoretical framework for the study of adult cognitive plasticity. Psychological Bulletin, 136(4), 659-76. [DOI:10.1037/a0020080] [PMID]

Lutz, J., Herwig, U., Opialla, S., Hittmeyer, A., Jäncke, L., \& Rufer, M., et al. (2014). Mindfulness and emotion regulation-an fMRI study. Social Cognitive and Affective Neuroscience, 9(6), 776-85. [DOI:10.1093/scan/nst043] [PMID] [PMCID]

Maguire, E. A., Gadian, D. G., Johnsrude, I. S., Good, C. D., Ashburner, J., \& Frackowiak, R. S. J., et al. (2000). Navigation-related structural change in the hippocampi of taxi drivers. Proceedings of the National Academy of Sciences, 97(8), 4398-403. [DOI:10.1073/ pnas.070039597] [PMID] [PMCID]

Maguire, E. A., Spiers, H. J., Good, C. D., Hartley, T., Frackowiak, R. S. J., \& Burgess, N. (2003). Navigation expertise and the human hippocampus: A structural brain imaging analysis. Hippocampus, 13(2), 250-9. [DOI:10.1002/hipo.10087] [PMID]

Mårtensson, J., Eriksson, J., Bodammer, N. C., Lindgren, M., Johansson, M., \& Nyberg, L., et al. (2012). Growth of language-related brain areas after foreign language learning. Neurolmage, 63(1), 240-4. [DOI:10.1016/j.neuroimage.2012.06.043] [PMID]

Mazziotta, J. C., Woods, R., Iacoboni, M., Sicotte, N., Yaden, K., \& Tran, M., et al. (2009). The myth of the normal, average human brain-The ICBM experience: (1) Subject screening and eligibility. NeuroImage, 44(3), 914-22. [DOI:10.1016/j.neuroimage.2008.07.062] [PMID] [PMCID]

McEwen, B. S., Nasca, C., \& Gray, J. D. (2015). Stress effects on neuronal structure: Hippocampus, amygdala, and prefrontal cortex. Neuropsychopharmacology, 41(1), 3-23. [DOI:10.1038/ npp.2015.171] [PMID] [PMCID]
Merriam, E. P., \& Colby, C. L. (2005). Active vision in parietal and extrastriate cortex. The Neuroscientist, 11(5), 484-93. [DOI:10.1177/1073858405276871] [PMID]

Mobbs, D., Marchant, J. L., Hassabis, D., Seymour, B., Tan, G., \& Gray, M., et al. (2009). From threat to fear: The neural organization of defensive fear systems in humans. The Journal of Neuroscience: The Official Journal of the Society for Neuroscience, 29(39), 12236-43. [DOI:10.1523/JNEUROSCI.2378-09.2009] [PMID] [PMCID]

Molenberghs, P., Ogilvie, C., Louis, W. R., Decety, J., Bagnall, J., \& Bain, P. G. (2015). The neural correlates of justified and unjustified killing: An fMRI study. Social Cognitive and Affective Neuroscience, 10(10), 1397-404. [DOI:10.1093/scan/nsv027] [PMID] [PMCID]

Mueller, K., Möller, H. E., Horstmann, A., Busse, F., Lepsien, J., \& Blüher, M., et al. (2015). Physical exercise in overweight to obese individuals induces metabolic- and neurotrophic-related structural brain plasticity. Frontiers in Human Neuroscience, 9, 372. [DOI:10.3389/fnhum.2015.00372] [PMID] [PMCID]

Niemann, C., Godde, B., Staudinger, U. M., \& Voelcker-Rehage, C. (2014). Exercise-induced changes in basal ganglia volume and cognition in older adults. Neuroscience, 281, 147-63. [DOI:10.1016/j.neuroscience.2014.09.033] [PMID]

Niemann, C., Godde, B., \& Voelcker-Rehage, C. (2016). Senior dance experience, cognitive performance, and brain volume in older women. Neural Plasticity, 2016, 9837321. [DOI:10.1155/2016/9837321] [PMID] [PMCID]

O'Hare, A. J., Dien, J., Waterson, L. D., \& Savage, C. R. (2008). Activation of the posterior cingulate by semantic priming: A co-registered ERP/fMRI study. Brain Research, 1189, 97-114. [DOI:10.1016/j.brainres.2007.10.095] [PMID]

Oden, K. B., Lohani, M., McCoy, M., Crutchfield, J., \& Rivers, S. (2015). Embedding emotional intelligence into military training contexts. Procedia Manufacturing, 3(Supplement C), 4052-9. [DOI:10.1016/j.promfg.2015.07.976]

Padival, M. A., Blume, S. R., Vantrease, J. E., \& Rosenkranz, J. A. (2015). Qualitatively different effect of repeated stress during adolescence on principal neuron morphology across lateral and basal nuclei of the rat amygdala. Neuroscience, 291(Supplement C), 128-45. [DOI:10.1016/j.neuroscience.2015.02.012] [PMID] [PMCID]

Pajonk, F. G., Wobrock, T., Gruber, O., Scherk, H., Berner, D., \& Kaizl, I., et al. (2010). Hippocampal plasticity in response to exercise in schizophrenia. Archives of General Psychiatry, 67(2), 133-43. [DOI:10.1001/archgenpsychiatry.2009.193] [PMID]

Palmer, T. D., Willhoite, A. R., \& Gage, F. H. (2000). Vascular niche for adult hippocampal neurogenesis. The Journal of Comparative Neurology, 425(4), 479-94. [DOI:10.1002/10969861(20001002)425:43.0.CO;2-3]

Paulus, M. P., Simmons, A. N., Fitzpatrick, S. N., Potterat, E. G., Van Orden, K. F., \& Bauman, J., et al. (2010). Differential brain activation to angry faces by elite warfighters: Neural processing evidence for enhanced threat detection. PloS One, 5(4), e10096. [DOI:10.1371/journal.pone.0010096] [PMID] [PMCID]

Pereira, A. C., Huddleston, D. E., Brickman, A. M., Sosunov, A. A., Hen, R., \& McKhann, G. M., et al. (2007). An in vivo correlate of exercise-induced neurogenesis in the adult dentate gyrus. Proceedings of the National Academy of Sciences of the United States 
of America, 104(13), 5638-43. [DOI:10.1073/pnas.0611721104] [PMID] [PMCID]

Perini, R., Bortoletto, M., Capogrosso, M., Fertonani, A., \& Miniussi, C. (2016). Acute effects of aerobic exercise promote learning. Scientific Reports, 6, 25440. [DOI:10.1038/srep25440] [PMID] [PMCID]

Phelps, E. A. (2004). Human emotion and memory: Interactions of the amygdala and hippocampal complex. Current Opinion in Neurobiology, 14(2), 198-202. [DOI:10.1016/j.conb.2004.03.015] [PMID]

Raine, A., \& Yang, Y. (2006). Neural foundations to moral reasoning and antisocial behavior. Social Cognitive and Affective Neuroscience, 1(3), 203-13. [DOI:10.1093/scan/ns1033] [PMID] [PMCID]

Raio, C. M., \& Phelps, E. A. (2015). The influence of acute stress on the regulation of conditioned fear. Neurobiology of Stress, 1 134-46. [DOI:10.1016/j.ynstr.2014.11.004] [PMID] [PMCID]

Raz, N., Gunning-Dixon, F., Head, D., Rodrigue, K. M., Williamson, A., \& Acker, J. D. (2004). Aging, sexual dimorphism, and hemispheric asymmetry of the cerebral cortex: Replicability of regional differences in volume. Neurobiology of Aging, 25(3), 377-96. [DOI:10.1016/S0197-4580(03)00118-0]

Rodman, H. R., \& Albright, T. D. (1987). Coding of visual stimulus velocity in area MT of the macaque. Vision Research, 27(12), 2035-48. [DOI:10.1016/0042-6989(87)90118-0]

Rovio, S., Spulber, G., Nieminen, L. J., Niskanen, E., Winblad, B., \& Tuomilehto, J., et al. (2010). The effect of midlife physical activity on structural brain changes in the elderly. Neurobiology of $\mathrm{Ag}$ ing, 31(11), 1927-36. [DOI:10.1016/j.neurobiolaging.2008.10.007] [PMID]

Ruegg, D. G., Kakebeeke, T. H., Gabriel, J.-P., \& Bennefeld, M. (2003). Conduction velocity of nerve and muscle fiber action potentials after a space mission or a bed rest. Clinical Neurophysiology, 114(1), 86-93. [DOI:10.1016/S1388-2457(02)00329-2]

Ruscheweyh, R., Willemer, C., Krüger, K., Duning, T., Warnecke, T., \& Sommer, J., et al. (2011). Physical activity and memory functions: An interventional study. Neurobiology of Aging, 32(7), 130419. [DOI:10.1016/j.neurobiolaging.2009.08.001] [PMID]

Sachdev, P. S., Lee, T., Wen, W., Ames, D., Batouli, A. H., \& Bowden, J., et al. (2013). The contribution of twins to the study of cognitive ageing and dementia: The Older Australian Twins Study. International Review of Psychiatry, 25(6), 738-47. [DOI:10.3109/09540 261.2013.870137] [PMID]

Schlaffke, L., Lissek, S., Lenz, M., Brüne, M., Juckel, G., \& Hinrichs, T., et al. (2014). Sports and brain morphology - a voxel-based morphometry study with endurance athletes and martial artists. Neuroscience, 259, 35-42. [DOI:10.1016/j.neuroscience.2013.11.046] [PMID]

Schubotz, R. I., \& von Cramon, D. Y. (2001). Functional organization of the lateral premotor cortex: fMRI reveals different regions activated by anticipation of object properties, location and speed. Cognitive Brain Research, 11(1), 97-112. [DOI:10.1016/S09266410(00)00069-0]

Sehm, B., Taubert, M., Conde, V., Weise, D., Classen, J., \& Dukart, J., et al. (2014). Structural brain plasticity in Parkinson's disease induced by balance training. Neurobiology of Aging, 35(1), 232-9. [DOI:10.1016/j.neurobiolaging.2013.06.021] [PMID]
Snowden, M., Steinman, L., Mochan, K., Grodstein, F., Prohaska, T. R., \& Thurman, D. J., et al. (2011). Effect of exercise on cognitive performance in community-dwelling older adults: review of intervention trials and recommendations for public health practice and research. Journal of the American Geriatrics Society, 59(4), 704-16. [DOI:10.1111/j.1532-5415.2011.03323.x] [PMID]

Steinbeis, N., Engert, V., Linz, R., \& Singer, T. (2015a). The effects of stress and affiliation on social decision-making: Investigating the tend-and-befriend pattern. Psychoneuroendocrinology, 62, 138-148. [DOI:10.1016/j.psyneuen.2015.08.003] [PMID]

Steinbeis, N., Engert, V., Linz, R., \& Singer, T. (2015b). The effects of stress and affiliation on social decision-making: Investigating the tend-and-befriend pattern. Psychoneuroendocrinology, 62, 138-48. [DOI:10.1016/i.psyneuen.2015.08.003] [PMID]

Stickgold, R., Malia, A., Maguire, D., Roddenberry, D., \& O'Connor M. (2000). Replaying the game: Hypnagogic images in normals and amnesics. Science, 290(5490), 350-3. [DOI:10.1126/science.290.5490.350] [PMID]

Tang, S. W., Chu, E., Hui, T., Helmeste, D., \& Law, C. (2008). Influence of exercise on serum brain-derived neurotrophic factor concentrations in healthy human subjects. Neuroscience Letters, 431(1), 62-5. [DOI:10.1016/j.neulet.2007.11.019] [PMID]

Thomas, A. G., Dennis, A., Rawlings, N. B., Stagg, C. J., Matthews, L., \& Morris, M., et al. (2016). Multi-modal characterization of rapid anterior hippocampal volume increase associated with aerobic exercise. NeuroImage, 131, 162-70. [DOI:10.1016/j.neuroimage.2015.10.090] [PMID] [PMCID]

Tran, V. (2013). Positive Affect Negative Affect Scale (PANAS) BT - Encyclopedia of Behavioral Medicine. In M. D. Gellman \& J. R. Turner (Eds.), Chapter 3, (pp. 1508-1509). New York, NY: Springer New York. [DOI:10.1007/978-1-4419-1005-9_978]

Trautmann, S. A., Fehr, T., \& Herrmann, M. (2009). Emotions in motion: Dynamic compared to static facial expressions of disgust and happiness reveal more widespread emotion-specific activations. Brain Research, 1284(Supplement C), 100-15. [DOI:10.1016/j. brainres.2009.05.075] [PMID]

van Praag, H. (2009). Exercise and the brain: Something to chew on. Trends in Neurosciences, 32(5), 283-90. [DOI:10.1016/j. tins.2008.12.007] [PMID] [PMCID]

van Praag, H., Kempermann, G., \& Gage, F. H. (2000). Neural consequences of enviromental enrichment. Nature Reviews Neuroscience, 1(3), 191-8. [DOI:10.1038/35044558] [PMID]

van Wingen, G. A., Geuze, E., Caan, M. W. A., Kozicz, T., Olabarriaga, S. D., \& Denys, D., et al. (2012). Persistent and reversible consequences of combat stress on the mesofrontal circuit and cognition. Proceedings of the National Academy of Sciences, 109(38) 15508-13. [DOI:10.1073/ pnas.1206330109] [PMID] [PMCID]

Voelcker-Rehage, C., Godde, B., \& Staudinger, U. M. (2011). Cardiovascular and coordination training differentially improve cognitive performance and neural processing in older adults. Frontiers in Human Neuroscience, 5, 26. [DOI:10.3389/fnhum.2011.00026] [PMID] [PMCID]

Voss, M. W., Vivar, C., Kramer, A. F., \& van Praag, H. (2013). Bridging animal and human models of exercise-induced brain plasticity. Trends in Cognitive Sciences, 17(10), 525-44. [DOI:10.1016/j. tics.2013.08.001] [PMID] [PMCID]

Wei, G., Zhang, Y., Jiang, T., \& Luo, J. (2011). Increased Cortical Thickness in Sports Experts: A Comparison of Diving Players 
with the Controls. PloS One, 6(2), e17112. [DOI:10.1371/journal. pone.0017112] [PMID] [PMCID]

Weinstein, A. M., Voss, M. W., Prakash, R. S., Chaddock, L., Szabo, A., \& White, S. M., et al. (2012). The association between aerobic fitness and executive function is mediated by prefrontal cortex volume. Brain, Behavior, and Immunity, 26(5), 811-9. [DOI:10.1016/j.bbi.2011.11.008] [PMID] [PMCID]

Yau, S., Gil-Mohapel, J., Christie, B. R., \& So, K. (2014). Physical exercise-induced adult neurogenesis: A good strategy to prevent cognitive decline in neurodegenerative diseases? BioMed Research International, 2014, 403120. [DOI:10.1155/2014/403120] [PMID] [PMCID] 
This Page Intentionally Left Blank 


\section{Research Paper: Health Promoting Self-care Behaviors in Patients With Multiple Sclerosis in the Southeast of Iran: Developing a Model for Practice}

Victoria Momenabadi $^{1}$ iD, Mohammad Hossein Kaveh ${ }^{1^{*}}$ (D), Nouzar Nakhaee ${ }^{2}$ (D), Kambiz Karimzadeh Shirazi ${ }^{3}$ (D), Behnaz Sedighi² ${ }^{2}$, Seyyed $^{-}$ Hamid Reza Tabatabaei ${ }^{4}$

1. Department of Health Education \& Promotion, School of Health, Shiraz University of Medical Sciences, Shiraz, Iran.

2. Neuroscience Research Center, Kerman University of Medical Sciences, Kerman, Iran.

3. Department of Health Education and Promotion, School of Health and Nutrition Sciences, Yasuj University of Medical Sciences, Yasuj, Iran

4. Department of Epidemiology, Research Center for Health Sciences, School of Health, Shiraz University of Medical Sciences, Shiraz, Iran.

\begin{tabular}{|l|l|}
\hline $\begin{array}{l}\text { Use yur devic to scan } \\
\text { and read thearticle online }\end{array}$ & $\begin{array}{l}\text { Citation: Momenabadi, V., Kaveh, M. H., Nakhaee, N., Karimzadeh Shirazi, K., Sedighi, B., \& Tabatabaei, S. H. R. (2020). } \\
\text { Health Promoting Self-care Behaviors in Patients With Multiple Sclerosis in the Southeast of Iran: Developing a Model for Prac- } \\
\text { tice. Basic and Clinical Neuroscience, 11(5), 687-700. http://dx.doi.org/10.32598/bcn.11.5.1670.1 }\end{array}$ \\
dol'http://dx.doi.org/10.32598/bcn.11.5.1670.1
\end{tabular}

Article info:

Received: 07 Jan 2019

First Revision: 25 Jan 2019

Accepted: 15 Sep 2019

Available Online: 01 Sep 2020

Keywords:

Self-care, Health promotion, Multiple Sclerosis, Structural equation model

\section{ABSTRACT}

Introduction: Promoting self-care practice, as a critical strategy for enhancing the quality of life in patients with Multiple Sclerosis (MS) is a challenging issue. This study aimed to propose a model of health-promoting self-care behaviors in MS patients.

Methods: In this cross-sectional study, 200 patients with MS, who had referred to the Hospital for Special Diseases in Kerman City, Iran, were chosen. The main data collection instruments were the multiple sclerosis knowledge questionnaire, the Rosenberg self-esteem scale, multiple sclerosis self-efficacy scale, questionnaire of perceived barriers and benefits of selfcare behaviors, social support, the health promotion lifestyle profile II, and resilience and sense of coherence scale. Data analysis was conducted in SPSS V. 22 and AMOS18 software. The Structural Equation Modeling (SEM) was also used for further analysis of data

Results: The model explained $82 \%$ of variance in Health-Promoting self-care Behavior (HPB). The results of the final model obtained from the SEM showed that self-efficacy $(\beta=0.53, \mathrm{SE}=0.04, \mathrm{P}=0.007)$, self-esteem $(\beta=0.39, \mathrm{SE}=0.04, \mathrm{P}=0.005)$, social support $(\beta=0.36$, $\mathrm{SE}=0.04, \mathrm{P}=0.009)$, sense of coherence $(\beta=0.34, \mathrm{SE}=0.07, \mathrm{P}=0.006)$, resilience $(\beta=0.33$, $\mathrm{SE}=0.07, \mathrm{P}=0.018)$, and perceived benefits $(\beta=0.25, \mathrm{SE}=0.05, \mathrm{P}=0.009)$ had a positive and significant relationship with HPB.

Conclusion: The self-care empowerment model in patients with MS presented in this study can be used as a framework for designing health promotion interventions to improve the quality of life of patients with MS. 


\section{Highlights}

- According to the final model of the path analysis, social support, perceived benefits, self-efficacy, and resilience with direct and indirect paths are significantly correlated with Health-Promoting self-care Behavior (HPB).

- Self-esteem was positively and indirectly correlated with HPB and the sense of coherence was directly correlated with HPB.

- In this regard, self-efficacy followed by self-esteem and social support are the most influential variables on HPB.

\section{Plain Language Summary}

Multiple Sclerosis (MS) affects all aspects of the individual's health, including physical, mental, and social dimensions. In addition to drug therapy, physicians and health staff should pay special attention to self-care in MS patients. The results of some studies on MS patients suggest that self-care programs reduce MS complications, such as depression and stress, boost self-esteem, provide an appropriate health-promoting model, satisfy basic health needs, increase the effectiveness of health education and health promotion programs, and contribute to understanding and defining the key elements of long-term behavior modification. The present study adopts a cross-sectional approach, using the main constructs of Simmons' health-promoting self-care behaviors model as well as important and effective self-care variables to present a model of Health-Promoting self-care Behavior (HPB) in MS patients. Path analysis was utilized to test the primary version of the conceptual model and yield a final model in addition to identifying the direct and indirect effects of each path and standardized effects. The final model revealed that self-esteem, social support, perceived benefits, self-efficacy, resilience, and sense of coherence were the main predictors of HPB. Therefore, this model can be adopted in interventions and self-care training programs for MS patients.

\section{Introduction}

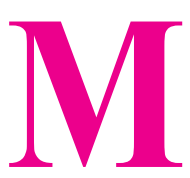

ultiple Sclerosis (MS) is one of the prevalent diseases of the $21^{\text {st }}$ century. It affects all aspects of an individual's health, including physical, mental, and social aspects (Mousavizadeh, Dastoorpoor, Naimi, \& Dohrabpour, 2018). The major physical problems of MS patients that should be managed are pain, weakness and fatigue, visual impairment, muscle spasm, urinary and stool incontinence (While, Ulman, \& Forbes, 2007). Demyelination of nerve fibers not only affects sensory and motor functions, but also brings about psychopathological symptoms (Haussleiter, Brüne, \& Juckel, 2009). Several studies have shown high levels of stress, low self-confidence, depression, and mental distress in these patients. About $50 \%-60 \%$ of patients suffer from depression and $25 \%-40 \%$ from anxiety and stress (Dennison, Moss-Morris, \& Chalder, 2009). These side effects, in general, influences the psychological health of these individuals. Thus, the patients need training on how to overcome these complications (Dennison et al., 2009; While et al., 2007). Physicians, health, and nursing staff should pay special attention to self-care in MS patients besides drug therapy (Amato \& Portaccio, 2012).
Evidence suggests that conscious self-care can help prevent and control many mental and physical consequences of chronic diseases and reduce their economic burden; therefore, special attention should be allocated to counseling and empowering patients (both physical and mental) (Adams et al., 2003).

Self-care is a practice in which a person, independent of others, take care of himself or herself (Naroie, Naji, , Abdeyazdan, \& Dadkani, 2012). The results of some studies on MS patients have shown that self-care programs alleviate MS complications, including depression, anxiety, and stress on the one hand, and boost self-esteem and emotional representation on the other hand (Hazhir F, Ahadi H, Pourshahbaz A, \& Rezaei M, 2012). Other studies have reported factors that can facilitate self-care behaviors in these patients. For example, people who have high self-efficacy and self-esteem believe that they can change their behavior and engage in self-care behaviors to promote their health and quality of life (Amtmann et al., 2012). One of the factors that improves self-care behaviors in patients with chronic disease is social support. Studies have shown that the sense of loneliness and perceived social support are related to the level of self-efficacy in patients. MS patients grow disconnected from society and lose social support, thus becoming more vulnerable to social disadvantages, economic crises, 
and physical and mental health problems (Fry \& Debats, 2012; Ghasemipour \& Nazai, 2012). Besides, the results of previous studies have shown a significant relationship between patients) improved awareness of self-care behaviors and positive changes in the disease, including diminished anxiety, and improved quality of life. Studies have also demonstrated the key role of the two components of resilience and sense of coherence in choosing a healthy lifestyle and adopting health-promoting self-care behaviors in patients with chronic MS (Luthar, Cicchetti, \& Becker, 2000; Scherer \& Bruce, 2001; WainWright et al., 2008).

Considering the above discussion about the factors influencing health-promoting self-care behaviors (self-efficacy, self-esteem, social support, perceived barriers and benefits, awareness, resilience, and sense of coherence), providing an appropriate health-promoting model and satisfying basic health needs will increase the effectiveness of health education and health promotion programs. These outcomes in turn contribute to understanding and defining the key elements for behavior modifications in the long-term (Estebsari et al., 2018).

A variety of theoretical models have been introduced for health-promoting behaviors by experts. In this context, a relatively comprehensive model called "the health-promoting self-care behaviors model" was proposed by Simmons. The model for health-promoting self-care behavior is one of the patterns that is commonly used in the field of behavior changes. This model presents a comprehensive conceptual model called the health-promoting self-care behavior model, which is based on the Orem self-care model (1985), the model of client health behavior (1982), and the Pender's health promotion model (1987). This background provides a broad and extensive framework for identifying and explaining hypothetical factors affecting decision-making, performance, design, and assessment of interventions (Simmons S, 1990a). Given the wide array of components included in Simmons' health-promoting self-care behaviors model, the model is primarily conceptual and hypothetical and draws on other theories and models. In this regard, it is fairly eclectic, making it a useful general guide for designing educational interventions. However, given the demographic variations and the types of chronic diseases, each subject must be matched concerning the constructs of Simmons' model. Also, as discussed in the introduction section, the role of other important factors such as social support, resilience, and the sense of coherence in health-promoting self-care behaviors in MS patients has been documented. Therefore, the present study, based on the main constructs of Simmons' healthpromoting self-care behaviors model as well as impor- tant and effective self-care variables, adopts a cross-sectional approach to present a model of health-promoting self-care behavior in MS patients.

\section{Materials and Methods}

\subsection{Study type and research method}

This cross-sectional study was undertaken in 2018 on 200 MS patients who had referred to Kerman Hospital for Special Diseases using a simple random sampling method. Kerman City, as the capital of Kerman Province, is the largest province of Iran. With a development rate of 0.251 , this province has been rated as one of the deprived areas of Iran (rank 10) in terms of health indicators (Yazdani \& Montazer, 2018). According to the latest Census in 2016, Kerman has a population of about 24738 (Tabatabaei et al., 2017).

\subsection{The inclusion and exclusion criteria}

The inclusion criteria consisted of medical diagnosis of the relapsing-remitting type of MS, disability scale (EDSS) below 5 (i.e. patients that are not at the stage of disability), an age range of 19 to 35 years, signing informed consent for participation in the study, ability to understand the content (minimum primary education) and complete the questionnaire.

The exclusion criteria included abandoning the study for any reason or contracting a disease that prevented the participation of the subjects in the study.

\subsection{Sample size}

Based on the sample size formula in the correlation studies (Hulley, Cummings, Browner, Grady, \& Newman, 2013), a sample of 173 was estimated with a confidence interval of $95 \%$ and the test power of $80 \%$. Considering a $10 \%$ probable loss, a final sample size of 200 was chosen. The patients were selected using a simple random sampling method.

\subsection{Data collection instruments}

The study data were collected using 10 self-report questionnaires, as described below.

1-Demographic Checklist: It contains demographic information such as age, sex, marital status, educational level, number of children, living area (city, village), employment status, and monthly income. 
2- Multiple Sclerosis Knowledge Questionnaire (MSKQ): It was designed by Giordano et al. in 2009. This questionnaire, which measures MS patients' knowledge about their illness, consists of 25 three-choice items (True, False, Neutral). The total score of the scale ranges between 0 and 25 (Giordano et al., 2010). After reviewing and introducing some modifications, a 26-item questionnaire was developed, which was suitable for the diagnostic status of patients with MS in Iran, and was validated. The total score of the scale ranged between 0 and 26 and it had a Cronbach alpha coefficient of 0.76 (Safarpour, 2014).

3- Rosenberg Self-Esteem Scale (RSE): This scale, designed by Rosenberg in 1965, contains 10 two-choice items (I agree, I disagree). The total score of the scale ranges between 0 and 10 (Rosenberg, 1965). It was validated for MS patients in Iran; this scale had a Cronbach alpha coefficient of 0.97 (Aghayi, Gozal, Zeinali, Ahmadi, 2017).

4- Multiple Sclerosis Self-efficacy Scale (MSSS): Designed by Rigby et al. in 2003, this scale includes 11 items that are rated on a 5-point Likert-type scale (strongly agree, agree, neutral, disagree, strongly disagree). The total score of the scale ranges between 11 and 55 points. The scale comprises three subscales of "independence and activity", "personal control", and "concerns and interests" (Rigby, Domenech, Thornton, Tedman, \& Young, 2003). This scale was validated for MS patients in Iran and its Cronbach alpha coefficient was reported as 0.90 (Tanhaye Reshvanlo \& Soleimanian, 2012).

5. The scale of perceived barriers in MS: This scale was designed by Morowati-Sharifabad et al. in 2016 to determine the perceived barriers to self-care activities in MS patients. It contains 12 items that are rated on a 3-point Likert-type scale (never, a little, a lot). The total score of the scale ranges between 0 and 24 with higher scores indicating higher perceived barriers to self-care health-promoting behaviors in patients with MS. The face and content validities of the questionnaire was confirmed by health education practitioners and specialists and its Cronbach alpha coefficient was reported as 0.86 (Morowatisharifabad, Momeni, Eslami, DehghaniTafti, \& Hakimzadeh, 2016).

6. The scale of perceived benefits in MS: This scale was also designed by Morowati Sharifabad et al. in Iran in 2016 to determine the perceived benefits of self-care activities in MS patients. It contained 6 items assessed on a 5-point Likert-type scale (totally agree, agree, neutral, disagree, totally disagree). The total score of the scale ranges between 6 and 30 points. The face and content validities of the scale were confirmed by health education specialists and its Cronbach alpha coefficient was reported as 0.79 (Morowati Sharifabad et al., 2016).

7- Connor-Davidson, Resilience scale (CD-RISC): Designed by Connor and Davidson in 2003, this scale includes 25 items that are assessed on a 5-point Likert scale (totally wrong, somewhat wrong, neutral, somewhat correct, totally correct). The total score ranges between 0 and 100 (Connor, \& Davidson, 2003). This scale has been validated in Iran and its Cronbach alpha coefficient was reported as 0.84 (Hagh Ranjbar, Kakavand Borjali, \& Bermas, 2011).

8- Sense of Coherence (SOC) Scale: Developed by Flensborg-Madsen et al. in 2006, this scale contains 35 questions in the form of multiple-choice questions (yes, no, I do not know). The total score of the scale ranges between 35 and 105 (Flensborg-Madsen, Ventegodt, \& Merrick, 2006). This scale has been validated in Iran and its Cronbach alpha coefficient was reported as 0.89 (Ehteshamzadeh, Sabrinazarzadeh, \& Mamarbashi, 2013).

9- Medical Outcomes Study-Social Support Survey (MOS-SSS): The survey was designed by Stewart et al. in 1991 to study medical consequences. It includes 19 questions rated on a 5-point Likert-type scale (never, often, sometimes, most often, always). The total score of the scale ranges between 19 and 95 points. It measures 5 aspects of social support: concrete support, emotional support, information, kindness, and positive social interaction (Stewart, Hays, \& Ware, 1988). The scale has been validated in Iran and its Cronbach alpha coefficient was reported as 0.97 (Mohammadzadeh, Sayehmiri, \& Mahmoudi, 2016).

10-Health-Promoting Lifestyle Profile II (HPLP II): It was developed by Walker et al. in 1995. This questionnaire includes 52 items that are assessed on a 4-point Likert scale (never, sometimes, often, and always) and measures 6 dimensions of physical activity, nutrition, spiritual growth, interpersonal relation, stress management, and health responsibility. The overall score of the scale ranges from 52 to 208 (Walker, Sechrist, \& Pender, 1995). This tool has been validated in MS patients in Iran and its Cronbach alpha coefficient was reported as 0.82 (Dashti-Dehkordi, Yousefi, Maghsoudi, Etemadifar, \& Maghsoudi, 2017)

In this study, the questionnaires validated in Iran, as described above, were used for data collection. Given that 


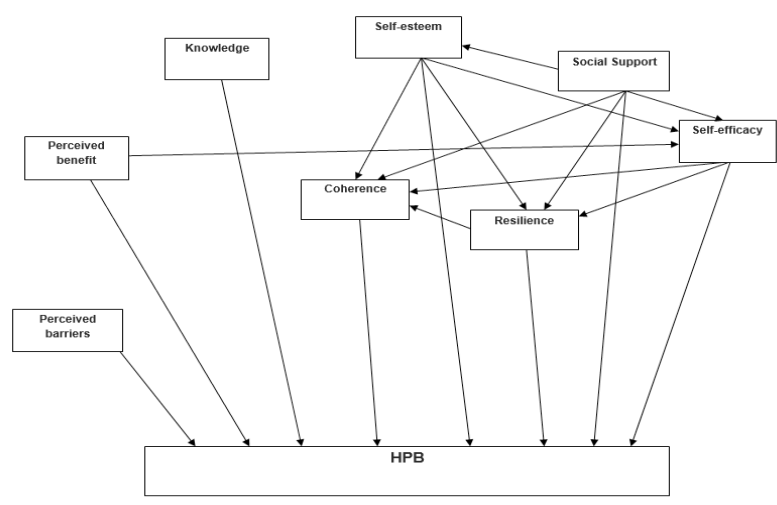

NEUR SCIENCE

Figure 1. Conceptual framework of health-promoting self-care behavior in MS patients (Ma et al., 2013; O'Brien, 1993; Simmons, 1990b; Wainwright et al., 2007)

multiple questionnaires had to be filled out by patients, to prevent fatigue and reduced accuracy in the target group, the scales were completed in two consecutive days.

\subsection{Ethical considerations}

After obtaining the necessary permits for conducting the research and making arrangements with the relevant authorities, written informed consent regarding the voluntary nature of participation in the study was obtained from MS patients. They were also ensured about the confidentiality of information, guaranteed that if the research was terminated, they would not suffer any physical or moral harm. Ethical approval was obtained from the Ethics Committee of Shiraz University of Medical Sciences (IR.SUMS.REC.1396.182) (Project No: 15554).

\subsection{Study analysis}

Before the analysis, the normality of the main research variables was assessed with the Shapiro-Wilk test. Data analysis was conducted in SPSS V. 22 (SPSS; Inc, Chicago, IL, USA) and AMOS18 ( AMOS: ADC, Chicago, IL, USA). Descriptive statistics, the Pearson correlation coefficient, and path analysis were used to test the primary version of the conceptual model, provide a final model and identify the direct and indirect effects of each path and standardized effects. During data analysis, various model indicators such as Comparative Fit Index (CFI), the Goodness of Fit Index (GFI) (above 0.9), Root Mean Square Error of Approximation (RMSEA) (below $0.08)$, and the Chi-square index/degrees of freedom $\left(\chi^{2}\right)$ df) (below 3) were evaluated, all of which indicate the suitability of the model. The primary conceptual model

Table 1. Descriptive statistics of theoretical constructs as potentials determinants of health-promoting self-care behaviors in the participants $(\mathrm{n}=200)$

\begin{tabular}{|c|c|c|c|c|}
\hline Variables & $\mathbf{N}$ & Mean \pm SD & Min. & Max. \\
\hline Perceived barriers & 200 & $13.13 \pm 4.8$ & 0.0 & 20.0 \\
\hline Perceived benefit & 200 & $21.4 \pm 4.5$ & 11.0 & 30.0 \\
\hline Knowledge & 200 & $14.1 \pm 4.4$ & 0.0 & 24.0 \\
\hline Self-esteem & 200 & $6.2 \pm 2.4$ & 0.0 & 10.0 \\
\hline Social Support & 200 & $59.6 \pm 17.6$ & 19.0 & 95.0 \\
\hline Self-efficacy & 200 & $31.8 \pm 7.3$ & 18.0 & 51.0 \\
\hline Coherence & 200 & $73.2 \pm 13.3$ & 40.0 & 103.0 \\
\hline Resilience & 200 & $53.7 \pm 18.7$ & 17.0 & 100.0 \\
\hline Health promoting self-care behaviors & 200 & $117.2 \pm 34.6$ & 52.0 & 208.0 \\
\hline
\end{tabular}




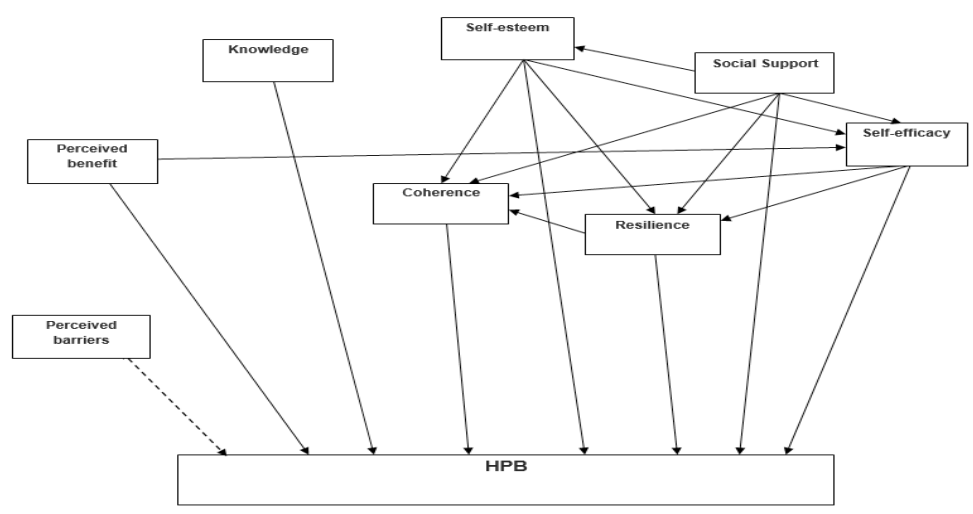

NEUR SCIENCE

Figure 2. The primary model of health-promoting self-care behavior based on the Pearson correlation results (Source: original data)

---- Significant relationship; --- Insignificant relationship

for improving health-promoting self-care behaviors in MS patients, based on the main constructs of the Simmons' health-promoting self-care behaviors model and review of the literature, is presented in Figure 1. The level of significance was less than 0.05 in all analyses.

\section{Results}

The Mean \pm SD age of the participants was $29.9 \pm 8.3$ years. Concerning gender, $67 \%$ of the subjects were female and the rest were male. In terms of education, $62.5 \%$ had a diploma and tertiary degrees, and the rest held high school or lower education. More than half of the participants were married (60\%). As for employment, $45 \%$ were employed and the rest were unemployed.
In this study, of 220 questionnaires distributed, 200 were completed and returned (response rate $=90.90 \%$ ). Of 20 subjects who failed to complete the questionnaire and withdrew from the study, 11 noted dissatisfactions and 9 physical problems as the main reason.

Table 1 presents the descriptive statistics of the constructs of the health-promoting self-care behaviors model. The mean values of the constructs of the healthpromoting self-care behavior model were as follows: perceived barriers (13.13), perceived benefits (21.4), knowledge (14.1), self-esteem (6.2), social support (59.6), self-efficacy (31.8), sense of coherence (73.2), resilience (53.7), and Health-Promoting self-care Behavior (HPB) (117.2).

Table 2. The correlation matrix of the health-promoting self-care behavior model $(n=200)$

\begin{tabular}{|c|c|c|c|c|c|c|c|c|c|}
\hline Variables & $\begin{array}{c}\text { Perceived } \\
\text { Barrier }\end{array}$ & $\begin{array}{c}\text { Perceived } \\
\text { Benefit }\end{array}$ & Knowledge & $\begin{array}{c}\text { Self- } \\
\text { esteem }\end{array}$ & $\begin{array}{c}\text { Social } \\
\text { Support }\end{array}$ & $\begin{array}{c}\text { Self- } \\
\text { efficacy }\end{array}$ & $\begin{array}{c}\text { Coher- } \\
\text { ence }\end{array}$ & $\begin{array}{l}\text { Resil- } \\
\text { ience }\end{array}$ & $\begin{array}{c}\text { Health-promot- } \\
\text { ing Self-care } \\
\text { Behavior }\end{array}$ \\
\hline Perceived barriers & 1 & & & & & & & & \\
\hline Perceived benefit & -0.018 & 1 & & & & & & & \\
\hline Knowledge & 0.039 & $0.504^{* *}$ & 1 & & & & & & \\
\hline Self-esteem & $-0.153^{*}$ & $0.575^{* *}$ & $0.503^{* *}$ & 1 & & & & & \\
\hline Social Support & $-0.145^{*}$ & $0.482 * *$ & $0.347^{* *}$ & $0.492 * *$ & 1 & & & & \\
\hline Self-efficacy & -0.123 & $0.663^{* *}$ & $0.570 * *$ & $0.754^{* *}$ & $0.629 * *$ & 1 & & & \\
\hline Coherence & -0.134 & $0.625^{* *}$ & $0.448 * *$ & $0.735^{* *}$ & $0.704^{* *}$ & $0.811^{* *}$ & 1 & & \\
\hline Resilience & -0.099 & $0.630^{* *}$ & $0.464^{* *}$ & $0.751^{* *}$ & $0.624^{* *}$ & $0.868^{* *}$ & $0.816^{* *}$ & 1 & \\
\hline $\begin{array}{l}\text { Health promoting } \\
\text { self-care behavior }\end{array}$ & -0.078 & $0.671^{* *}$ & $0.483^{* *}$ & $0.716^{* *}$ & $0.675^{* *}$ & $0.846^{* *}$ & $0.854^{* *}$ & $0.842^{* *}$ & 1 \\
\hline
\end{tabular}

${ }^{*} \mathrm{P}<0.001 ; * * \mathrm{P}<0.05$ 


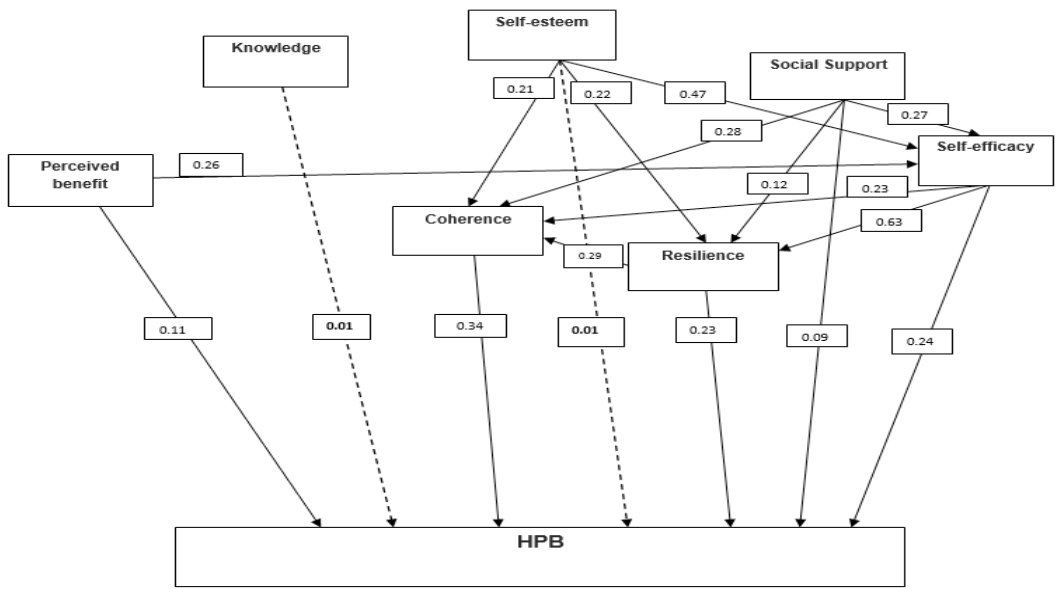

NEUR SCIENCE

Figure 3. The modified model of pathway analysis for health-promoting self-care behaviors (Source: Original data)

-_-- Significant relationship; - - - Insignificant relationship

As the results of the descriptive analysis suggest, the mean scores of the predictor constructs of self-care behaviors in MS patients were higher than $50 \%$ of the maximum construct scores. Also, the mean score of the patients' HPB was 117.2 , which is more than $50 \%$ of the maximum score (104), indicating that the participants of our study were in desirable conditions in this respect.

According to the correlation matrix, the correlation coefficient was between 0.018 and 0.868 . The sense of coherence $(r=0.854)$ and perceived barriers $(r=-0.078)$ exhibited the strongest and weakest correlations with the HPB variable. Based on the results of the Pearson correlation coefficient, perceived benefits, knowledge, selfesteem, social support, self-efficacy, sense of coherence, and resilience, except for perceived barriers, had a direct and significant relationship with the HPB response variable at the significant level of $\mathrm{P}<0.001$ (Table 2). Therefore, perceived barriers were removed from the primary hypothetical model (Figure 2) and the model fitness was assessed using the path analysis.

In the analysis of the modified model, the results of path analysis suggested that the perceived benefits, sense of coherence, resilience, social support, and self-efficacy had a direct and significant relationship with the HPB variable. However, the variables of knowledge and selfesteem were not directly correlated with the HPB variable (Figure 3). Therefore, the modified model of the path analysis was re-fitted after removing the insignificant variables.

The final model of the path analysis is shown in Figure 4. We tested the goodness of fit of the final model. The
CFI, GFI, RMSEA, and $\chi^{2} / \mathrm{df}$ were estimated at 1.00 , $0.99,0.04$, and 1.28 , respectively; the results exhibit the desirable fitness of the model.

In the final study of the path analysis, the results show that social support, perceived benefits, and self-efficacy and resilience directly or indirectly were positively correlated with HPB (Table 3). The standardized beta $(\beta)$ coefficients from the direct path indicate that for each unit increase in social support, perceived benefits, selfefficacy, and resilience, the mean HPB score increases to $0.09,0.11,0.24$, and 0.23 , respectively, and vice versa (Figure 4).

The self-esteem variable illustrated a positive and significant correlation with HPB only indirectly $(\beta=0.39$, $\mathrm{SE}=0.04, \mathrm{P}=0.005$ ). The sense of coherence variable also showed a positive and significant correlation with HPB only with a direct path $(\beta=0.34, \mathrm{SE}=0.07, \mathrm{P}=0.006)$. In this regard, considering their total effect on HPB, selfefficacy, followed by self-esteem and social support (Table 3 ) are the most influential variables, respectively. Table 3 presents further details of the final model.

Finally, the above model suggests that $82 \%$ of variations in the HPB variable $\left(\mathrm{R}^{2}=0.82\right)$ can be explained by six constructs of self-esteem, social support, perceived benefits, self-efficacy, resilience, and sense of coherence. The remaining $18 \%$ is defined by other variables.

\section{Discussion}

The health-promoting self-care behaviors are the major factors affecting the ability of MS patients, stimulating 


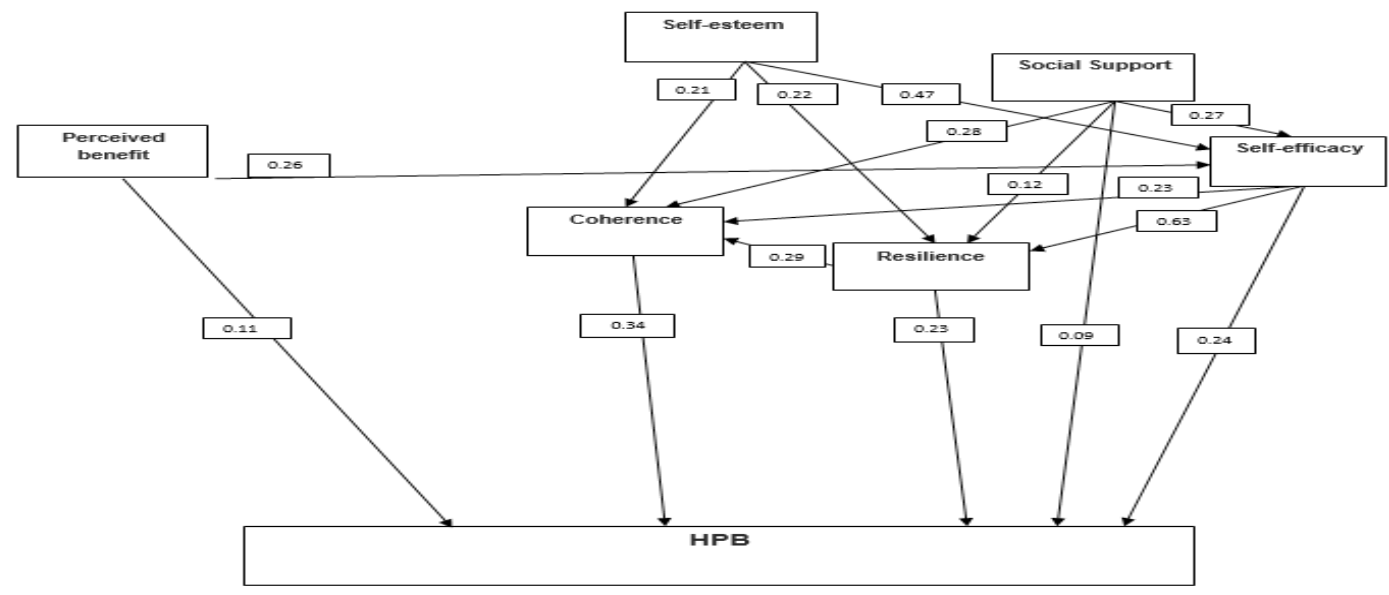

Figure 4. The final model of pathway analysis of health-promoting self-care behavior

NEUR SCIENCE

Numbers indicate the standardized path coefficient of the model (Source: original data); Significant relationship

repressed, and forgotten capabilities that empower them to overcome their disabilities (Masoudi, Mohammadi, Nabavi, \& Ahmadi, 2008). The present study revealed an overall good fit between the proposed model and the data. According to the results, self-efficacy, self-esteem, social support, sense of cohesion, resilience, and perceived benefits are the main predictors of health-promoting self-care behaviors among which self-efficacy exerted the highest effect.

Based on the results of this study, $67 \%$ of MS patients were female. This finding is consistent with those reported in previous studies according to which the female patients outnumber the male patients by 3 to 4 times (Mousavizadeh et al., 2018; Sedaghat, Jessri, Behrooz, Mirghotbi, \& Rashidkhani, 2016). The higher rate of MS among women may be related to their physiological features such as sex hormones and stress. Additionally, the growing incidence of MS in females suggests that hormonal differences make women more susceptible to environmental risk factors (Izadi, Nikseresht, Poursadeghfard, Borhanihaghighi, \& Heydari, 2015).

Like similar studies, the mean age of participants in this research was 29.9 years (Mousavizadeh et al., 2018). This may be due to a variety of adolescence-related factors, such as education, university entrance exam, occupation, or marriage, which are major determinants of one's future life and failure in any of these areas could be a source of stress.

The number of married participants in this study was twice that of single adults. This finding may be prompted by factors related to marriage and childbearing, which guide individuals into important stages of life, and failure to adapt to new situations may be a source of stress and anxiety, which are major predictors of MS. Also, more than $50 \%$ of patients were university students or had a university degree, which is in agreement with the findings of Abedini et al. (2016). The results of studies have exhibited that the effect of education on health is greater than that of income and occupation, as illiteracy can be linked to a sense of irresponsibility concerning health and treatment. Moreover, people who have a university degree are more likely to appreciate the effect of health-promoting self-care behaviors (Karimy, Abedi, Amin-Shokravi, \& Tavafian, 2013).

Furthermore, the current study suggests a positive and significant correlation between self-efficacy and HPB. The results of the path analysis model exhibited that, both in the direct and the indirect paths, with one unit increase in self-efficacy, the mean HPB score rose by 0.24 and 0.28 , respectively. Also, according to the results, the self-efficacy variable exerts the highest effect on HPB, which is in agreement with the results of previous studies (Finn, 2006; Polsingchan , 2010; Salahshoori et al., 2015). In a 2007 study by Wu et al. on patients with type 2 diabetes, health-promoting self-care behaviors were found to be in a positive and significant relationship with the duration of the disease, the outcome expectation, and self-efficacy of patients. The researchers concluded that self-efficacy improvement models contribute to the adoption of health-promoting self-care behaviors (Wu et al., 2007). In his model of self-promotion, Pender found that self-efficacy was one of the major predictors 
Table 3. Directions and standard coefficients of the pathway model

\begin{tabular}{|c|c|c|c|c|c|c|c|c|c|}
\hline \multirow{2}{*}{ Pathway } & \multicolumn{3}{|c|}{ Total Effect } & \multicolumn{3}{|c|}{ Indirect Effect } & \multicolumn{3}{|c|}{ Direct Effect } \\
\hline & $\beta^{*}$ & SE & $\mathbf{P}$ & $\beta^{*}$ & SE & $\mathbf{P}$ & $\beta^{*}$ & SE & $\mathbf{P}$ \\
\hline Self-esteem $>$ HPB & 0.39 & 0.04 & $0.005^{*}$ & 0.39 & 0.04 & $0.005^{¥}$ & --- & --- & --- \\
\hline Social Support> HPB & 0.36 & 0.04 & $0.009^{¥}$ & 0.28 & 0.04 & $0.009^{¥}$ & 0.09 & 0.04 & $0.047^{*}$ \\
\hline Perceived benefit> HPB & 0.25 & 0.05 & $0.009^{*}$ & 0.14 & 0.03 & $0.007^{¥}$ & 0.11 & 0.04 & $0.008^{*}$ \\
\hline Self-efficacy> HPB & 0.53 & 0.04 & $0.007^{¥}$ & 0.28 & 0.05 & $0.032^{¥}$ & 0.24 & 0.06 & $0.007^{*}$ \\
\hline Resilience> HPB & 0.33 & 0.07 & $0.018^{*}$ & 0.10 & 0.03 & $0.004^{¥}$ & 0.23 & 0.07 & $0.020^{*}$ \\
\hline Coherence> HPB & 0.34 & 0.07 & $0.006^{¥}$ & - - - & - - - & $\ldots$ & 0.34 & 0.07 & $0.006^{*}$ \\
\hline
\end{tabular}

$\chi^{2} / \mathrm{df}=1.28, \mathrm{GFI}=0.99, \mathrm{CFI}=1.00, \mathrm{IFI}=1.00, \mathrm{RFI}=0.98, \mathrm{NFI}=1.00, \mathrm{RMSEA}=0.037$, Hoelter Index=590

NEUR SCIENCE

HPB: Health-promoting self-care behavior; $x^{2} /$ df: Chi-squared/degree of freedom; GFI: The goodness of fit index; CFI: Comparative fit index; IFI: Incremental fit index; RFI: Relative fit index; NFI: Normed fit index; RMSEA: Root mean square error of approximation; * Standardized beta; ${ }^{*}$ P values are significant.

of health. Moreover, $86 \%$ of studies on the health promotion model has endorsed the role of self-efficacy as a predictor of behavior (Pender, Murdaugh, \& Parsons, 2006). However, Wendling et al. found no significant relationship between patients' self-efficacy and their selfcare behaviors (Wendling \& Beadle, 2015). This may be due to the diversity of research environments and subjects, or data gathering tools.

The current findings indicate a positive and significant correlation between self-esteem and HPB. According to the results of path analysis, in an indirect path, with improved self-esteem, the mean HPB score rises by 0.39 , which is consistent with the literature. For example, the study of Madani et al. on 38 patients with MS revealed a significant relationship between the self-esteem of patients and health-promoting self-care behaviors. Patients with a higher sense of self-esteem were more likely to adopt health-promoting self-care behaviors to deal with their problems, while patients with low self-esteem tended to adopt emotionally-focused oppositional methods (Madani, Navipour, \& Rouzbayani, 2008). Also, in a study on the relationship between self-esteem, social support, and oppositional behavior in MS patients, O'Brien found a significant relationship between selfesteem and problem-oriented oppositional methods, including health-promoting behaviors. Moreover, the necessity of assessing self-esteem, social support, and coping behavior in patients with MS has been emphasized (O'Brien, 1993). Health-promoting self-care behaviors are one of the coping behaviors in MS patients that encourages patients to take responsibility for their health (Acton \& Malathum, 2000). This correlation, however, was not observed in the study of Sinclair et al. and Von Bothmer et al. (Sinclair \& Scroggie, 2005; Von Bothmer \& Fridlund, 2005). This discrepancy of results could be attributed to different research environments, subjects, or data gathering tools.

Based on these findings, there was a positive and significant correlation between social support and HPB. Hence, the results of the path analysis model revealed that both in the direct and the indirect paths, one unit increase in the social support variable raised the mean HPB score by 0.92 and 0.28 , respectively. In practice, a high level of social support can improve health-promoting self-care behaviors. In this context, other studies have demonstrated the positive impact of social support on HPB (Alizadeh, Keshavarz, Mirghafourvand, \& Zayeri, 2018; Ballard, 2009). However, the study of Yi Ps et al. has not reported a relationship between perceived social support and HPB (Pan, Cameron, Desmeules, Morrison, Craig, Jiang, 2009). This discrepancy of results could be attributed to different research environments or data gathering tools. Social support is critical to health promotion as it accelerates physical and emotional needs, protecting individuals against stressful living conditions (Ballard, 2009). It is, therefore, important to boost social support (financial, emotional, and informational) in MS patients to promote HPB and reduce the wide range of problems associated with the disease, including financial problems linked to the purchase of drugs and emotional problems (Mazaheri, Fanian, \& Zargham-Boroujeni, 2011). 
The results also exhibit a positive correlation between the sense of coherence and HPB. In the direct path, a unit increase in the sense of coherence augmented the average HPB score by 34.4. Other researchers reported similar results. For example, in a pilot study on students in Finland, physical activity was found to be positively related to the sense of coherence (Hassmen, Koivula, \& Uutela, 2000). Another study also suggested that a low sense of coherence was strongly linked to smoking behaviors (Glanz, Maskarinec, \& Carlin, 2005).

In the present study, a positive and significant correlation was observed between resilience and HPB. The results of the path analysis model revealed that both in the direct and the indirect paths, one unit increase in the resilience raised the mean HPB score by 0.23 and 0.10 , respectively. In this regard, the 2013 study of Chang on patients with chronic renal disease reported a positive and significant relationship between resilience and HPB dimensions (Ma et al., 2013).

Since two constructs of resilience and sense of coherence are predictable, problem-oriented decision-making interventions can be employed to help individuals learn how to come to terms and cope with their diseases. Particularly, stress management training for MS patients can affect both the sense of coherence and the degree of patients' resilience. In other words, in chronic diseases, it is essential to help patients set a target for recovery, hamper the progression of the disease, strengthen positive thinking and the adoption of a positive attitude towards life and future, and mitigate disappointment (as studies have shown that disappointment in chronic patients exerts influence on resilience). In the same vein, the ability to communicate with others and establish useful social networks are fundamental as expanded communication network precludes isolation and reduces depression and frustration in patients (Dayapoğlu, \& Tan, 2012; Valizadeh, Sohrabnejad, Mehraban, \& Ahmadbokani, 2014).

According to the results, perceived benefits are also positively and significantly correlated with HPB. Moreover, the results of the final path analysis model indicated that the standardized coefficients of direct and indirect paths of perceived benefits of HPB were 0.19 and 0.14 , respectively. In other words, with increased perceived benefits, the mean HPB score soared by 0.19 and 0.14 , which is statistically significant. In this regard, several studies have reported the significant correlation between perceived benefits and health-promoting self-care behavior in chronic diseases (Aalto \& Uutela, 1997; Koch, 2002), though this correlation was not observed in the study of Gillibrand \& Stevenson, (2006). This discrep- ancy of results could be attributed to different research environments and or data gathering tools.

\subsection{Study strengths and limitations}

This is the first study to adopt SEM to test a comprehensive theoretical model that combines the constructs of Simons' health-promoting self-care behavior model and other constructs derived from the literature review as predictors of self-promoting self-care behaviors in MS patients in Iran. The new model presented in the study, i.e. "self-care empowerment model in MS patients", demonstrates strong predictors of health-promoting selfcare behaviors (both direct and indirect) in MS patients. It is one of the major strengths of this study besides its innovation.

Our study also had several limitations. One major limitation of the study was the reluctance of some patients to participate in the project. Another limitation was concerned with data gathering based on patients' self-report, which precluded accurate observations by the researcher. Therefore, an objective review and periodic follow-ups might yield different outcomes. The last limitation had something to do with the nature of MS disease and the large number of questions, which made the completion of questionnaires an exhausting task for some patients.

It is suggested that future interventional studies on promoting self-care behaviors in patients with MS based on the above model as well as qualitative studies identify other predictors of health-promoting self-care behavior. Moreover, since it was not possible to evaluate health status in the research environment and patients' residence, it is recommended that future research considers this issue to account for marginalized patients.

\section{Conclusion}

The study findings revealed that a higher level of selfefficacy, self-esteem, social support, sense of coherence, resilience, and perceived benefits improved health-promoting self-care behaviors in MS patients. The current study also suggested that besides the main constructs of the Simmons model, factors such as resilience, sense of coherence, and social support play significant roles in the adoption of health-promoting self-care behaviors. Therefore, these factors should be considered by health professionals and physicians in designing and implementing programs for MS patients. In conclusion, this model of health promotion is recommended as a way of improving self-care behaviors in MS patients. 


\section{Ethical Considerations}

Compliance with ethical guidelines

The study was approved by the Ethics Committee of Shiraz University of Medical Sciences (Ref: IR.SUMS. REC.1396.182) and written consent forms were taken from all participants.

\section{Funding}

The research project was financially supported by Shiraz University of Medical Sciences, Shiraz, Iran (Grant No: 15554).

\section{Authors' contributions}

All authors were equally contributed in preparing this article.

\section{Conflict of interest}

The authors declared no conflict of interest.

\section{References}

Aalto, A. M., \& Uutela, A. (1997). Glycemic control, self-care behaviors, and psychosocial factors among insulin treated diabetics: A test of an extended health belief model. International Journal of Behavioral Medicine, 4(3), 191. [DOI:10.1207/ s15327558ijbm0403_1] [PMID]

Abedini, M., Paksersht, M., Rafiei, A., Valadan, R., Amjadi, O., \& Khajavi, R., et al. (2016). Demographic and clinical characteristics of multiple sclerosis. Journal of Mazandaran University of Medical Sciences, 25(132), 13-22. http://jmums.mazums.ac.ir/ article-1-6725-en.pdf

Acton, G. J., \& Malathum, P. (2000). Basic need status and healthpromoting self-care behavior in adults. Western Journal of Nursing Research, 22(7), 796-811. [DOI:10.1177/01939450022044764] [PMID]

Adams, A. S., Mah, C., Soumerai, S. B., Zhang, F., Barton, M B., \& Ross-Degnan, D. (2003). Barriers to self-monitoring of blood glucose among adults with diabetes in an HMO: A cross sectional study. BMC Health Services Research, 3(1), 1-8. [DOI:10.1186/1472-6963-3-6] [PMID] [PMCID]

Aghayi, M., Gozal, S., Zeinali, M., Ahmadi, N., \& Mirzaei, S. (2017). [The efficiency of problem-solving skills on quality of life and self-esteem of patients with multiple sclerosis (Persian)]. Journal of Psychology New Ideas, 1(1), 29-38. http://jnip. ir/article-1-49-fa.html

Alizadeh, T., Keshavarz, Z., Mirghafourvand, M., \& Zayeri, F. (2018). Investigation of health promoting lifestyle and social support and their correlation among Iranian women with multiple sclerosis. International Journal of Women's
Health and Reproduction Sciences, 6(2), 167-73. [DOI:10.15296/ ijwhr.2018.28]

Amato, M. P., \& Portaccio, E. (2012). Management options in multiple sclerosis-associated fatigue. Expert Opinion on Pharmacotherapy, 13(2), 207-216. [DOI:10.1517/14656566.2012.6477 67] [PMID]

Amtmann, D., Bamer, A. M., Cook, K. F., Askew, R. L., Noonan, V. K., \& Brockway, J. A. (2012). University of Washington self efficacy scale: A new self-efficacy scale for people with disabilities. Archives of Physical Medicine and Rehabilitation, 93(10), 1757-65. [DOI:10.1016/j.apmr.2012.05.001] [PMID]

Ballard FA. (2009). Homeless sheltered women's health promotion behaviors., The University of North Carolina at Greensboro. https://libres.uncg.edu/ir/uncg/f/Ballard uncg_0154D_10116.pdf

Connor, K. M., \& Davidson, J. R. (2003). Development of a new resilience scale: The Connor-Davidson resilience scale (CDRISC). Depression and Anxiety, 18(2), 76-82. [DOI:10.1002/ da.10113] [PMID]

Dashti-Dehkordi, A., Yousefi, H., Etemadifar, M., \& Maghsoudi, J. (2017). Health promotion behaviors and its correlation with some of the demographic factors in patients with multiple sclerosis. Advances in Nursing \& Midwifery, 26(93), 11-18. https://onlinelibrary.wiley.com/doi/abs/10.1002/da.10113

Dayapoğlu, N., \& Tan, M. (2012). Evaluation of the effect of progressive relaxation exercises on fatigue and sleep quality in patients with multiple sclerosis. The Journal of Alternative and Complementary Medicine, 18(10), 983-987. [DOI:10.1089/ acm.2011.0390] [PMID] [PMCID]

Dennison, L., Moss-Morris, R., \& Chalder, T. (2009). A review of psychological correlates of adjustment in patients with multiple sclerosis. Clinical Psychology Review, 29(2), 141-153. [DOI:10.1016/j.cpr.2008.12.001] [PMID]

Ehteshamzadeh P, Sabrinazarzadeh R, \& Mamarbashi M (2013). The relationship between sense of coherence and job performance with intermediation strategis of coping with stress and mental health. Psychological Methods and Models Journal, 3(13), 85-97. https://www.sid.ir/en/journal/ViewPaper.aspx?id $=411402$

Estebsari, F., Dastoorpoor, M., Mostafaei, D., Khanjani, N., Khalifehkandi, Z. R., \& Foroushani, A. R., et al. (2018). Design and implementation of an empowerment model to prevent elder abuse: A randomized controlled trial. Clinical Interventions in Aging, 13, 669. [DOI:10.2147/CIA.S158097] [PMID] [PMCID]

Finn, A. (2006). The relationship between self-efficacy and health promoting behaviors, illness severity, mental health and perceived quality of life in multiple sclerosis patients Modern Psychological Studies, 11(2), 11-24. https://scholar.utc $\mathrm{edu} / \mathrm{mps} / \mathrm{vol11} /$ iss2/3/

Flensborg-Madsen, T., Ventegodt, S., \& Merrick, J. (2006). Sense of coherence and physical health. Testing antonovsky's theory. The Scientific World Journal, 6, 640524. [DOI:10.1100/ tswhhm.2006.232]

Fry, P. S., \& Debats, D. L. (2002). Self-efficacy beliefs as predictors of loneliness and psychological distress in older adults. The International Journal of Aging and Human Development, 55(3), 233-269. [DOI:10.2190/KBVP-L2TE-2ERY-BH26] [PMID] 
Ghasemipour, M., Nazai, H. (2012). [The relationship between quality of life and social support in Multiple Sclerosis patients (Persian)]. Nursing Developement in Health, 3(4-5). http://ndhj. lums.ac.ir/article-1-91-fa.html

Gillibrand, R., \& Stevenson, J. (2006). The extended health belief model applied to the experience of diabetes in young people. British Journal of Health Psychology, 11(1), 155-69. [DOI:10.1348/135910705X39485] [PMID]

Giordano, A., Messmer Uccelli, M., Pucci, E., Martinelli, V., Borreani, C., \& Lugaresi, A., et al. (2010). The Multiple Sclerosis Knowledge Questionnaire: a self-administered instrument for recently diagnosed patients. Multiple Sclerosis Journal, 16(1), 100-11.[DOI:10.1177/1352458509352865] [PMID]

Glanz, K., Maskarinec, G., \& Carlin, L. (2005). Ethnicity, sense of coherence, and tobacco use among adolescents. Annals of Behavioral Medicine, 29(3), 192-9. [DOI:10.1207/ s15324796abm2903_5] [PMID]

Hagh, R. F., Kakavand, A. R., Borjali, A., \& Bermas, H. (2011). Resiliency and life quality in mothers of children with mental retardation. Journal of Health Psychology, 1(1), 177-87. https:// www.sid.ir/en/journal/ViewPaper.aspx?ID=360993

Hassmen, P., Koivula, N., \& Uutela, A. (2000). Physical exercise and psychological well-being: A population study in Finland. Preventive Medicine, 30(1), 17-25. [DOI:10.1006/ pmed.1999.0597] [PMID]

Haussleiter, I. S., Brüne, M., \& Juckel, G. (2009). Psychopathology in multiple sclerosis: Diagnosis, prevalence and treatment. Therapeutic advances in neurological disorders, 2(1), 13-29. [DOI:10.1177/1756285608100325] [PMID] [PMCID]

Hazhir, F., Ahadi, H., Pourshahbaz, A., Rezaei, M. (2012). The effectiveness of cognitive-behavior therapy on illness representations of multiple-sclerosis and improving their emotional states. Journal of Kermanshah University of Medical Sciences, 15(6), 415-428. https://sites.kowsarpub.com/jkums/ articles/78893.html

Hulley SB, Cummings SR, Browner WS, Grady DG, \& Newman TB. (2013). Designing clinical research : an epidemiologic approach. (3th ed. ed.). Philadelphia,: PA: Lippincott Williams \& Wilkins.

Izadi, S., Nikseresht, A. R., Poursadeghfard, M., Borhanihaghighi, A., \& Heydari, S. T. (2015). Prevalence and incidence of multiple sclerosis in Fars province, Southern Iran Iranian Journal of Medical Sciences, 40(5), 390. [PMCID] [PMID]

Karimy, M., Abedi, A., Amin-Shokravi, F., \& Tavafian, S. S. (2013). Preventing hiv transmission among the opiate-dependent population in zarandieh: Evaluation of the hbmbased educational programs. Health Education \& Health Promotion, 1(1), 21-31. https://hehp.modares.ac.ir/article5-3781-en.pdf

Koch, J. (2002). The role of exercise in the African-American woman with type 2 diabetes mellitus: Application of the health belief model. Journal of the American Academy of Nurse Practitioners, 14(3), 26-30. [DOI:10.1111/j.1745-7599.2002. tb00103.x] [PMID]

Luthar, S. S., Cicchetti, D., \& Becker, B. (2000). The construct of resilience: A critical evaluation. Development and Psychopathology, 12(4), 857-85. [DOI:10.1017/S0954579400004156] [PMID] [PMCID]
Ma, L. C., Chang, H. J., Liu, Y. M., Hsieh, H. L., Lo, L., Lin, M. Y., \& Lu, K. C. (2013). The relationship between healthpromoting behaviors and resilience in patients with chronic kidney disease. The Scientific World Journal, 2013. [DOI:10.1155/2013/124973] [PMID] [PMCID]

Ma, L. C., Chang, H. J., Liu, Y. M., Hsieh, H. L., Lo, L., Lin, M. Y., \& Lu, K. C. (2013). The relationship between healthpromoting behaviors and resilience in patients with chronic kidney disease. The Scientific World Journal, 2013. [DOI:10.1155/2013/124973] [PMID] [PMCID]

Madani, H., Navipour, H., \& Rouzbayani, P. (2008). Relations between self-esteem and applying coping mechanism and self-care program in Multiple Sclerosis (MS) patients. Journal of Ilam University of Medical Sciences, 15(4), 31-41. https:/ www.sid.ir/en/journal/ViewPaper.aspx?ID=108782

Masoudi, R., Mohammadi, E., Nabavi, S. M., \& Ahmadi, F (2008). The effect of Orem based self-care program on physical quality of life in multiple sclerosis patients. Journal of Shahrekord Uuniversity of Medical Sciences, 10(2), 9-21. http://journal.skums.ac.ir/article-1-5-fa.htm

Mazaheri, M., Fanian, N., \& Zargham-Boroujeni, A. (2011). Experiences of patients with multiple sclerosis from group counseling. Iranian Journal of Nursing and Midwifery Research 16(2), 181. [PMCID] [PMID]

Mohammadzadeh, J., \& Sayehmiri, K. (2016). [Standardization of social support scale (MOS) of adults who have chronic diseases in Ilam, 2015 (Persian)]. Scientific Journal of Ilam University of Medical Sciences, 23(7), 69-77. [http://sjimu.medilam. ac.ir/article-1-2483-fa.html]

MorowatiSharifabad, M. A., Momeni, Z., Eslami, M. H., DehghaniTafti, A., \& Hakimzadeh, A. (2016). [Study of Factors Associated with Self-Care bhaviors in patients with Multiplesclerosis in Yazd City based on health belief model (Persian)]. Tolooebehdasht, 15(2), 82-93. http://tbj.ssu.ac.ir/ article-1-2189-fa.html

Mousavizadeh, A., Dastoorpoor, M., Naimi, E., \& Dohrabpour K. (2018). Time-trend analysis and developing a forecasting model for the prevalence of multiple sclerosis in Kohgiluyeh and Boyer-Ahmad Province, Southwest of Iran. Public Health 154, 14-23. [DOI:10.1016/j.puhe.2017.10.003] [PMID]

Naroie, S., Naji, S. A., Abdeyazdan, G. H., \& Dadkani, E. (2012) Effect of applying self-care orem model on quality of life in the patient under hemodialysis. Zahedan Journal of Research in Medical Sciences, 14(1), 8-12. https://sites.kowsarpub.com/ zjrms/articles/93608.html

O'Brien, M. T. (1993). Multiple sclerosis: the relationship among self-esteem, social support, and coping behavior. Applied Nursing Research, 6(2), 54-63. [DOI:10.1016/S08971897(05)80103-X]

Pender, N. J., Murdaugh, C. L., \& Parsons, M. A. (2006). Health promotion in nursing practice. New Jersey: Prentice Hall https:// books.google.com/books?id=jVJqAAAAMAAJ\&q

Polsingchan S. (2010). Health-promoting behaviors in Thai persons with chronic renal failure [PhD. thesis]. Texas: The University of Texas at Austin. http:// citeseerx.ist.psu.edu/viewdoc/down load?doi=10.1.1.834.1195\&rep=rep1\&type=pdf

Rigby, S., Domenech, C., Thornton, E., Tedman, S., \& Young, C. (2003). Development and validation of a self-efficacy meas- 
ure for people with multiple sclerosis: The Multiple Sclerosis Self-efficacy Scale. Multiple Sclerosis Journal, 9(1), 73-81. [DOI:10.1191/1352458503ms870oa] [PMID]

Rosenberg, M. (1965). Rosenberg Self-Esteem Scale (RSE). Acceptance and commitment therapy. Measures Package, 61, 52. [DOI:10.1037/t01038-000]

Safarpour Y. (2014). [Evaluation of knowledge of patients with Multiple Sclerosis in case Multiple Sclerosis patients referring to Imam Hossein Hospital in 2014 (Persian)]. [MD. thesis]. Tehran: Shahid Beheshti University of Medical sciences.

Salahshoori, A., Harooni, J., Salahshouri, S., Hassanzadeh, A., Mostafavi, F., \& Molaei, M. (2015). Investigation on association between self-efficacy, perceived barriers and social supports with health promoting behaviors in elderly in Dena city. Health System Research, 11(1), 30-42. http://hsr.mui.ac.ir/ article-1-763-fa.html

Scherer, Y. K., \& Bruce, S. (2001). Knowledge, attitudes, and self-efficacy and compliance with medical regimen, number of emergency department visits, and hospitalizations in adults with asthma. Heart $\mathcal{E}$ Lung, 30(4), 250-7. [DOI:10.1067/ mhl.2001.116013] [PMID]

Sedaghat, F., Jessri, M., Behrooz, M., Mirghotbi, M., \& Rashidkhani, B. (2016). Mediterranean diet adherence and risk of multiple sclerosis: A case-control study. Asia Pacific Journal of Clinical Nutrition, 25(2), 377-84. http://apjcn.nhri.org.tw/ server/APJCN/25/2/377.pdf

Simmons,S.J.(1990). Thehealth-promoting self-caresystemmodel: Directions for nursing research and practice. Journal of $A d-$ vanced Nursing, 15(10), 1162-6.[DOI:10.1111/j.1365-2648.1990. tb01708.x] [PMID]

Simmons,S.(1990b). Thehealth-promotingSelf-caresystemModel: Directions for nursing researh and practice. Journal of Advanced Nursing, 15(10), 1162-6. [DOI:10.1111/j.1365-2648.1990. tb01708.x] [PMID]

Sinclair, V. G., \& Scroggie, J. (2005). Effects of a cognitive-behavioral program for women with multiple sclerosis. Journal of Neuroscience Nursing, 37(5), 249-57. [DOI:10.1097/01376517200510000-00004] [PMID]

Stewart, A. L., Hays, R. D., \& Ware, J. E. (1988). The MOS short-form general health survey: Reliability and validity in a patient population. Medical Care, 26(7), 724-35. [DOI:10.1097/00005650-198807000-00007] [PMID]

Tabatabaei, S. V. A., Ardabili, H. E., Haghdoost, A. A., Dastoorpoor, M., Nakhaee, N., \& Shams, M. (2017). Factors affecting physical activity behavior among women in Kerman Based on the Theory of Planned Behavior (TPB). Iranian Red Crescent Medical Journal, 19(10). [DOI:10.5812/ircmj.14057]

Tanhaye Reshvanlo, F., \& Soleimanian, A. (2012). [Psychometric examination of Multiple Sclerosis Self-Efficacy Scale (Persian)]. The Journal of Applied Behavioral Science, 12(1), 9-18.

Valizadeh R, Sohrabnejad S, Mehraban S, \& Ahmadbokani S. (2014). [Effectiveness of cognitive-behavioral based stress management training on depression of MS female patients (Persian)]. Scientific Journal of Ilam University of Medical Sciences, 23(5), 91-8. https://www.sid.ir/en/Journal/ViewPaper. aspx?ID $=482595$
Von Bothmer, M. I., \& Fridlund, B. (2005). Gender differences in health habits and in motivation for a healthy lifestyle among Swedish university students. Nursing and Health Sciences, 7(2), 107-18. [DOI:10.1111/j.1442-2018.2005.00227.x] [PMID]

Wainwright, N. W., Surtees, P. G., Welch, A. A., Luben, R. N., Khaw, K. T., \& Bingham, S. A. (2007). Healthy lifestyle choices: could sense of coherence aid health promotion? Journal of Epidemiology \& Community Health, 61(10), 871-6. [DOI:10.1136/ jech.2006.056275] [PMID] [PMCID]

Walker, S., Sechrist, K., \& Pender, N. (1995). The health-promoting lifestyle profile II.

Wendling, S., \& Beadle, V. (2015). The relationship between self-efficacy and diabetic foot self-care. Journal of Clinical $\mathcal{E}$ Translational Endocrinology, 2(1), 37-41. [DOI:10.1016/j. jcte.2015.01.001] [PMID] [PMCID]

While, A., Ullman, R., \& Forbes, A. (2007). Development and validation of a learning needs assessment scale: A continuing professional education tool for multiple sclerosis specialist nurses. Journal of Clinical Nursing, 16, 1099-8. [DOI:10.1111/ j.1365-2702.2007.01693.x] [PMID]

Wu, S. F. V., Courtney, M., Edwards, H., McDowell, J., Shortridge-Baggett, L. M., \& Chang, P. J. (2007). Self-efficacy, outcome expectations, and self-care behaviour in people with type 2 diabetes in Taiwan. Journal of Clinical Nursing, 16(11c), 250-7. [DOI:10.1111/j.1365-2702.2006.01930.x] [PMID]

Yazdani, M. H., \& Montazer, F. (2017). [Analysis of indicators of health status in provinces and ten regions of Iran (Persian)] Health and Development Journal, 6(4), 290-301. http://jhad. kmu.ac.ir/article-1-419-fa.html

Pan, S. Y., Cameron, C., Desmeules, M., Morrison, H., Craig, C. L., \& Jiang, X. (2009). Individual, social, environmental, and physical environmental correlates with physical activity among Canadians: A cross-sectional study. BMC Pub Health, 9:21. [DOI:10.1186/1471-2458-9-21] [PMID] [PMCID] 
This Page Intentionally Left Blank 


\title{
Research Paper: Psychological, Neurophysiological, and Mental Factors Associated With Gamma-Enhancing Neurofeedback Success
}

\author{
Zeynab Khodakarami $^{1^{*}}$ (D), Mohammad Firoozabadi² ${ }^{2}$ (C)
}

1. Department of Biomedical Engineering, Science and Research Branch, Islamic Azad University, Tehran, Iran

2. Department of Medical Physics, Faculty of Medical Sciences, Tarbiat Modares University, Tehran, Iran

\begin{tabular}{l|l}
$\begin{array}{l}\text { use vour device to scan } \\
\text { and read the article online }\end{array}$ & $\begin{array}{l}\text { Cittation: Khodakarami, Z., \& Firoozabadi, M. (2020). Psychological, Neurophysiological, and Mental Factors Associated } \\
\text { With Gamma-Enhancing Neurofeedback Success. Basic and Clinical Neuroscience, 11(5), 701-714. http://dx.doi.org/10.32598/ } \\
\text { bcn.11.5.1878.1 }\end{array}$ \\
dol'http://dx.doi.org/10.32598/bcn.11.5.1878.1
\end{tabular}

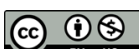

Article info:

Received: 27 May 2019

First Revision: 10 Jun 2019

Accepted: 02 Oct 2020

Available Online: 01 Sep 2020

Keywords:

Neurofeedback, BrainComputer Interface (BCI)illiteracy, Performance prediction, Fluid intelligence, Mental strategies

\begin{abstract}
$\underline{\text { A B S T RA C T }}$
Introduction: Regarding the neurofeedback training process, previous studies indicate that $10 \%-50 \%$ of subjects cannot gain control over their brain activity even after repeated training sessions. This study is conducted to overcome this problem by investigating inter-individual differences in neurofeedback learning to propose some predictors for the trainability of subjects.

Methods: Eight healthy female students took part in 8 (electroencephalography) EEG neurofeedback training sessions for enhancing EEG gamma power at the $\mathrm{Oz}$ channel. We studied participants' preexisting fluid intelligence and EEG frequency sub-bands' power during 2-min eyes-closed rest and a cognitive task as psychological and neurophysiological factors, concerning neurofeedback learning performance. We also assessed the self-reports of participants about mental strategies used by them during neurofeedback to identify the most effective successful strategies.
\end{abstract}

Results: The results revealed that a significant percentage of individuals ( $25 \%$ in this study) cannot learn how to control their brain gamma activity using neurofeedback. Our findings suggest that fluid intelligence, gamma power during a cognitive task, and alpha power at rest can predict gamma-enhancing neurofeedback performance of individuals. Based on our study, neurofeedback learning is a form of implicit learning. We also found that learning without a user's mental efforts to find out successful mental strategies, in other words, unconscious learning, lead to more success in gamma-enhancing neurofeedback.

Conclusion: Our results may improve gamma neurofeedback efficacy for further clinical usage and studies by giving insight about both non-trainable individuals and effective mental strategies.

\section{* Corresponding Author:}

Zeynab Khodakarami, MSc.

Address: Department of Biomedical Engineering, Science and Research Branch, Islamic Azad University, Tehran, Iran.

Tel: +98 (912) 5072015

E-mail: z.khodakarami@gmail.com 


\section{Highlights}

- A comprehensive literature review on the relationship between personal factors and neurofeedback success.

- We introduced some predictors for Gamma-enhancing neurofeedback performance of individuals.

- We proposed effective instructions for Gamma-enhancing neurofeedback learning.

\section{Plain Language Summary}

Using neurofeedback technique, people can learn to modulate their brain neural activity voluntarily, through a realtime feedback of their EEG and a reward to further motivate them. Neurofeedback has been proven to be efficient both in treatment of many different mental disorders and in cognitive enhancement of healthy participants. After almost six decades of neurofeedback research, some serious challenges in this area, such as neurofeedback-illiteracy or highly dependence of neurofeedback on user's individual characteristics, still remain unsolved and controversial. It has been shown that a non-negligible proportion of participants (estimated $10 \%-50 \%$ ) fail to gain significant control over their brain function through neurofeedback, even after several training sessions, which leads to useless and frustrating expenses. In this study, we introduced some predictors for estimating the success of individuals in learning neurofeedback before starting this procedure, as well as some guidelines for use in training procedure.

\section{Introduction}

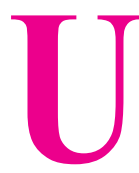

sing the neurofeedback technique, people can learn to modulate voluntarily their brain neural activity, through realtime feedback of their Electroencephalography (EEG) in the form of visual, auditory, or tactile information, and a reward during desirable changes in it. Using neurofeedback, different EEG frequency sub-bands activities could be modified (Berner, Schabus, Wienerroither, \& Klimesch, 2006; Keizer, Verment, \& Hommel, 2010; Leins et al., 2007; Raymond, Varney, Parkinson, \& Gruzelier, 2005; van Boxtel et al., 2012; Vernon et al., 2003). Neurofeedback has been proven to be efficient in the therapy of many different mental disorders such as attention-deficit hyperactivity disorder (Gevensleben et al., 2009), schizophrenia (Gruzelier, 2000), and anxiety (Moore, 2000). Also improved artistic (Egner \& Gruzelier, 2003; Raymond, Sajid, Parkinson, \& Gruzelier, 2005), sport-related (Arns, Kleinnijenhuis, Fallahpour, \& Breteler, 2008; Landers et al., 1991), and cognitive (Angelakis et al., 2007; Egner \& Gruzelier, 2004) functioning due to neurofeedback has been demonstrated in healthy participants.

Despite all advantages of neurofeedback, it has a main drawback. Gaining considerable long-lasting control on brain activity through neurofeedback requires a high number of training sessions, leading to high temporal, financial, and staff costs. Besides, previous studies re- vealed that a non-negligible proportion of participants (estimated 10\%-50\%) fail to gain significant control over their brain function through neurofeedback, even after several training sessions (Blankertz et al., 2010; Drechsler et al., 2007; Hammer et al., 2012; Hanslmayr, Sauseng, Doppelmayr, Schabus, \& Klimesch, 2005; Huster, Mokom, Enriquez-Geppert, \& Herrmann, 2013; Kober, Witte, Ninaus, Neuper, \& Wood, 2013; Lubar, Swartwood, Swartwood, \& O’Donnell, 1995; Neumann \& Birbaumer, 2003; Weber, Köberl, Frank, \& Doppelmayr, 2011). This phenomenon, first seen in Brain-Computer Interface (BCI) research, is called BCI-illiteracy (Blankertz et al., 2010; Kober et al., 2013; Witte, Kober, Ninaus, Neuper, \& Wood, 2013) or BCI-inefficiency (Hammer et al., 2012; Kübler, Blankertz, Müller, \& Neuper, 2011) in the literature, and has been demonstrated for both healthy and unhealthy participants, as for all EEG training features. In this context, the participants with and without the ability to learn self-regulation have been named as responders/non-responders, performers/ non-performers, or low/high aptitude users, respectively. Solving the BCI-illiteracy problem to avoid frustrating and costly training procedures is one of the biggest challenges in neurofeedback studies (Blankertz et al., 2010).

To solve the BCI-illiteracy problem, some research studies have been conducted in recent years to investigate whether there is any relationship between interindividual differences and the ability of people to learn neurofeedback self-regulation. Such studies aimed to propose some predictors for neurofeedback trainability. 
Table1. Summary of studies indicating the significant relationship between personal factors and neurofeedback performance

\begin{tabular}{|c|c|c|c|c|}
\hline & Personal Factor & EEG Trained Sub-Band & Study Population & Study \\
\hline \multirow{5}{*}{ 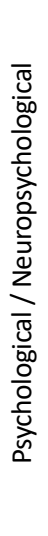 } & Measures of attention and memory span & Slow cortical potentials & Epilepsy patients & Daum et al. (1993) \\
\hline & Locus of control with regard to technology & Sensorimotor rhythm & Healthy subjects & Burde \& Blankertz (2006) \\
\hline & Intelligence quotient & Slow cortical potentials & $\begin{array}{l}\text { Amyotrophic lateral } \\
\text { sclerosis patients }\end{array}$ & Drechsler et al. (2007) \\
\hline & Motivational factors & $\begin{array}{l}\text { P300 / Sensorimotor } \\
\text { rhythm }\end{array}$ & $\begin{array}{l}\text { Amyotrophic lateral } \\
\text { sclerosis patients }\end{array}$ & Nijboer et al. (2010) \\
\hline & Locus of control with regard to technology & Sensorimotor rhythm & Healthy subjects & Witte et al. (2013) \\
\hline \multirow{3}{*}{ 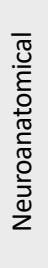 } & $\begin{array}{l}\text { Gray and white morphology of the } \\
\text { midcingulate cortex }\end{array}$ & Theta & Healthy subjects & Enriquez-Geppert et al. (2013) \\
\hline & $\begin{array}{l}\text { Structural integrity and myelination qual- } \\
\text { ity of deep white matter structures }\end{array}$ & Sensorimotor rhythm & Healthy subjects & Halder et al. (2013) \\
\hline & Gray and white matter volumes & $\begin{array}{l}\text { Sensorimotor rhythm/ } \\
\text { Gamma }\end{array}$ & Healthy subjects & Ninaus et al. (2015) \\
\hline \multirow{3}{*}{ 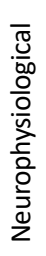 } & $\begin{array}{l}\text { Sensorimotor rhythm amplitude under } \\
\text { "relax with eyes open" }\end{array}$ & Sensorimotor rhythm & Healthy subjects & Blankertz et al. (2010) \\
\hline & $\begin{array}{l}\text { Low beta amplitude at eyes-open rest } \\
\text { / Beta-1 amplitude in the first 4.5-min } \\
\text { training block }\end{array}$ & Beta/Theta ratio & Healthy subjects & Nan et al. (2015) \\
\hline & Alpha amplitude at rest & Alpha & Healthy subjects & Wan et al. (2014) \\
\hline \multirow{3}{*}{ 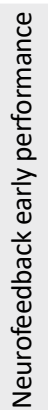 } & $\begin{array}{l}\text { Initial performance in Slow cortical } \\
\text { potentials self-regulation }\end{array}$ & Slow cortical potentials & $\begin{array}{l}\text { Amyotrophic lateral } \\
\text { sclerosis patients }\end{array}$ & Neumann \& Birbaumer (2003) \\
\hline & $\begin{array}{l}\text { Slow cortical potentials self-control in } \\
\text { the training session } 3\end{array}$ & Slow cortical potentials & $\begin{array}{l}\text { Amyotrophic lateral } \\
\text { sclerosis patients / } \\
\text { healthy subjects }\end{array}$ & Kübler et al. (2004) \\
\hline & $\begin{array}{c}\text { Sensorimotor rhythm self-control in the } \\
\text { training session } 9\end{array}$ & Sensorimotor rhythm & Healthy subjects & Weber et al. (2011) \\
\hline
\end{tabular}

Finding such factors that may serve to predict neurofeedback performance, on the one hand, results in a better understanding of both BCI-illiteracy phenomenon and underlying neurophysiological and behavioral mechanisms involved in brain self-regulation (Blankertz et al., 2010; Neumann \& Birbaumer, 2003), and on the other hand, until the problem of BCI-illiteracy is solved, utilizing neurofeedback alternatives for participants with less probability of success, may serve to avoid the costly and frustrating procedures. Previous studies have introduced predictors for neurofeedback learning perfor- mance based on four following factors (a summary can be found in Table 1):

1. Psychological/neuropsychological traits (Burde \& Blankertz, 2006; Daum et al., 1993; Drechsler et al., 2007; Hammer et al., 2012; Nijboer, Birbaumer, \& Kübler, 2010; Witte et al., 2013).

2. Brain structural and neuroanatomical factors (Enriquez-Geppert et al., 2013; Halder et al., 2013; Ninaus et al., 2015); 
3. Brain neurophysiological parameters in the form of brain activity (Blankertz et al., 2010; Nan, Wan, Vai, \& Da Rosa, 2015; Wan, Nan, Vai, \& Rosa, 2014);

4. Neurofeedback early training performance (Kübler, Neumann, Wilhelm, Hinterberger, \& Birbaumer, 2004; Neumann \& Birbaumer, 2003; Weber et al., 2011).

Mental strategies used by an individual during a neurofeedback session to gain self-control is another core feature of successful training. A proper mental strategy can facilitate and accelerate neurofeedback learning. In typical BCI applications, very specific instructions can be transmitted to the participants by the experimenters (Kober et al., 2013). But the mental strategies used in neurofeedback studies by participants and their effects are scarcely investigated (Kober et al., 2013; Nan et al., 2012). In the few studies conducted to assess the effectiveness of different mental strategies, positive strategies (e.g. positive thinking or positive emotions) and also no conscious and explicit mental strategy has been proven as successful ones (Angelakis et al., 2007; Birbaumer, Ruiz, \& Sitaram, 2013; Kober et al., 2013; Nan et al., 2012; Neumann \& Birbaumer, 2003; Rubik, 2011; Witte et al., 2013). Table 2 presents a summary of the studies that explored successful mental strategies in neurofeedback learning. As can be seen from both Table 1 and Table 2, most studies investigated the association between personal factors and neurofeedback performance have been focused on Sensorimotor Rhythms (SMR) and Slow Cortical Potentials (SCP) self-regulation, while personal factors associated with success in self-regulation of other EEG sub-band activities have seldom been studied.

The ability of individuals to enhance EEG gamma power using neurofeedback and subsequently improvement of focused arousal, memory, visual feature binding, and fluid intelligence have been reported before (Bird, Newton, Sheer, \& Ford, 1978; Elliott \& Müller, 1998; Keizer, et al., 2010; Keizer, Verschoor, Verment, \& Hommel, 2010; Khodakarami \& Firoozabadi, 2014; Rubik, 2011; Sams, 1995; Staufenbiel, Brouwer, Keizer, \& van Wouwe, 2014; Tallon-Baudry, Bertrand, Peronnet, \& Pernier, 1998). Visual feature binding phenomenon plays a key role in human visual perception (Hommel, 1998). Gamma neurofeedback illiteracy and possible effects of personal factors on it have rarely been investigated. This study aimed to investigate the relationship between inter-individual differences and gamma neurofeedback trainability of subjects. To do this, we examined the association between some psychological, neurophysiological, and mental factors and neurofeedback success in both trainable and non-trainable individuals, to define some reliable predictors for gamma-enhancing neurofeedback performance.

\section{Materials and Methods}

\subsection{Study participants}

Eight healthy female students voluntarily participated in this study (Mean \pm SD age: $24.46 \pm 2.35$ years; 2 lefthanded). All participants were neurofeedback- and BCInovices and had a normal or corrected-to-normal vision, with no history of neurological diseases (e.g. migraine or epilepsy) and no medication use that could influence cognitive performance or brain activity during the study. Before the experiments, all participants were informed about the purpose of the study and gave written informed consent to the study protocol. This study conforms with the World Medical Association Declaration of Helsinki.

\subsection{Study procedure}

All subjects participated in a pre-test neurophysiological and psychological factors assessment session. In that session, a 2-min baseline EEG was recorded during both eyes-closed rest and a cognitive task (pre-test baseline). The cognitive task was based on mental search and counting upward blue numbers among red numbers represented on a 17-inch computer screen, one meter away from the subject. After the baseline EEG recordings, the subject's fluid intelligence was measured by using Cattell's Culture-Fair Intelligence Test (Cattell, Krug, $\&$ Barton, 1973). At the beginning of the session before performing the intelligence test, the subjects filled out a questionnaire asked about their last night's sleep, health issues, and whether they are mentally prepared for participating in a psychological test (pre-test questionnaire). The pre-test sessions were conducted at 9-11 AM, at the neurofeedback lab of the Biomedical Engineering Department of the Science and Research Branch of Islamic Azad University.

After the end of all pre-test sessions, each subject participated in 8 gamma-enhancing neurofeedback sessions. At each session, before the start of neurofeedback training, a 2-min baseline EEG at eyes-closed rest was recorded from the subject (pretraining baseline), identical to the pre-test EEG recordings. Subjects were also asked to fill out two pretraining and posttraining questionnaires at the beginning and the end of each session, respectively. In the pretraining questionnaire, any notable changes possibly due to neurofeedback training in appetite, sleep pattern, memory capacity, and mental and cognitive ability during previous days were questioned. The posttraining questionnaire enquired for any mental strategies the subject applied for gaining control over the feedback during the session, and the success of them on a 
Table 2. Summary of studies suggesting effective mental strategies for neurofeedback success

\begin{tabular}{|c|c|c|c|}
\hline \multirow{2}{*}{ Successful Strategies } & EEG Trained Sub-band & Study Population & \multirow{2}{*}{ Study } \\
\hline & \multicolumn{2}{|c|}{ Mental Strategy (Personal Factor) } & \\
\hline $\begin{array}{l}\text { Neither instructions nor explicit mental } \\
\text { strategies }\end{array}$ & $\begin{array}{l}\text { Review on real-time functional mag- } \\
\text { netic resonance imaging neurofeed- } \\
\text { back of different bands }\end{array}$ & $\begin{array}{l}\text { Healthy subjects / } \\
\text { Animals }\end{array}$ & Birbaumer et al. (2013) \\
\hline No conscious mental strategy & Slow cortical potentials & $\begin{array}{l}\text { Amyotrophic lateral } \\
\text { sclerosis patients }\end{array}$ & $\begin{array}{c}\text { Neumann \& Birbaumer } \\
(2003)\end{array}$ \\
\hline No conscious mental strategy & Sensorimotor rhythm & Healthy subjects & Witte et al. (2013) \\
\hline $\begin{array}{l}\text { Positive strategies (positive thoughts) } \\
\text { and no strategy }\end{array}$ & Alpha & Healthy subjects & Angelakis et al. (2007) \\
\hline Positive thinking & Alpha & Healthy subjects & Nan et al. (2012) \\
\hline Positive emotions (e.g. happiness) & Gamma & Healthy subjects & Rubik (2011) \\
\hline No mental strategy & Sensorimotor rhythm & Healthy subjects & Kober et al. (2013) \\
\hline
\end{tabular}

5-point Likert-type scale (0: No control, 5: Full control). In posttraining questionnaires, the subjects again rated their motivation for gaining control over the feedback, on a 5-point Likert-type scale ( 0 : Not at all motivated, 5: Extremely motivated). An overview of the experimental design of the current study can be found in Figure 1.

\subsection{Intelligence assessment}

Fluid intelligence was tested by using the paper-based version of Cattell's Culture-Fair Intelligence Test (Scale 3) Form A (Cattell et al., 1973). This is a non-verbal and culture-independent test, with 50 multiple choice questions and 14-min test time. The test is composed of four individually timed subtests of series, classification, matrices, and conditions, with questions of increasing difficulty. The results of the test were calculated in terms of accuracy for each subject.

\subsection{Baseline EEG recordings}

Both pre-test and pretraining baseline EEG signals were recorded from the $\mathrm{Oz}$ electrode according to the international 10-20 system using BioGraph Infiniti

A)

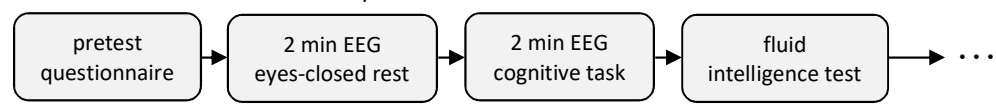

B)

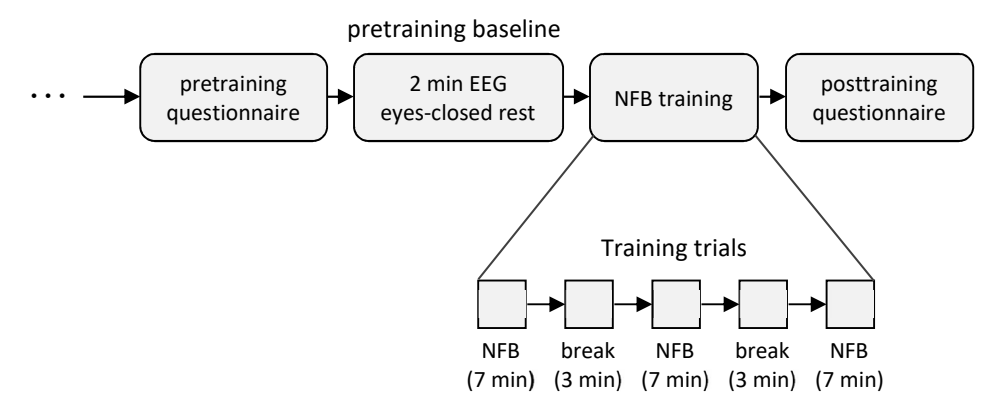

8 Gamma-enhancing neurofeedback sessions:

Figure 1. Schematic representation of the study experimental design

NEUR

A: The procedure of the pre-test session; B: The procedure of a single neurofeedback training session. Each subject participated in 8 gamma-enhancing neurofeedback sessions, three training sessions per week. 
Table 3. Descriptive statistics of study participants.

\begin{tabular}{|c|c|c|c|c|c|}
\hline \multirow{2}{*}{ Variable } & \multicolumn{2}{|c|}{ All $(n=8)$} & \multirow[t]{2}{*}{ Responders ( $n=6$ ) } & \multirow[t]{2}{*}{ Non-Responders $(n=2)$} & \multirow{2}{*}{ P (Mann-Whitney) ${ }^{\mathrm{b}}$} \\
\hline & Mean士SD & $P(K-S)^{a}$ & & & \\
\hline Age, y & $24.46 \pm 2.35$ & 0.20 & $24.05 \pm 2.61$ & $25.69 \pm 0.74$ & 0.50 \\
\hline $\mathrm{BMI}, \mathrm{kg} / \mathrm{m}^{2}$ & $20.65 \pm 2.62$ & 0.20 & $19.46 \pm 1.47$ & $24.19 \pm 1.93$ & $0.04^{*}$ \\
\hline IQ & $103.50 \pm 22.58$ & 0.20 & $112.17 \pm 18.33$ & $77.50 \pm 9.19$ & $0.04 *$ \\
\hline Gamma changec & - & - & $2.24 \pm 1.52$ & $-0.29 \pm 0.07$ & $0.04 *$ \\
\hline
\end{tabular}

${ }^{a}$ Results from the K-S test assessing the normal distribution of variables;

NEURCIENCE

${ }^{b}$ Results from the Mann-Whitney U test examining significant differences between responders and non-responders groups;

${ }^{c}$ Mean gamma power change from pretraining to training over 8 neurofeedback sessions $\left(\mu \mathrm{V}^{2}\right)$;

${ }^{*} \mathrm{P}<0.05$.

(Thought Technology Ltd, Montreal, QC) and golden electrodes, sampled at $256 \mathrm{~Hz}$. The ground electrode was located at the right earlobe, and the reference was at the left earlobe. Electrode impedances were kept below $5 \mathrm{k} \Omega$. During EEG recordings, the subjects were sitting still in a comfortable chair with arms lying relaxed on armrests.

\subsection{Neurofeedback training}

Eight gamma-enhancing neurofeedback sessions were conducted for each subject, three training sessions per week. Each training session consisted of three 7-min trials, with 3-min breaks between. In each trial, the EEG signal recorded from the $\mathrm{Oz}$ electrode (according to the international 10-20 system) was filtered to gamma (36$44 \mathrm{~Hz}$ ) and beta $(12-20 \mathrm{~Hz})$ frequency bands using two elliptic filters. Then, gamma and beta signal peak-to-peak amplitudes fed real-time back to the subject, with the aim of gamma enhancing and beta suppression simultaneously. We defined gamma frequency band around 40 $\mathrm{Hz}$ because it is the most widely accepted indicator of the gamma range (Başar-Eroglu, Strüber, Schürmann, Stadler, \& Başar, 1996; Hammond, 2000; Sams, 1995; Tallon-Baudry \& Bertrand, 1999). It has been suggested in previous studies that the beta frequency band power will be changed because of the gamma frequency band power changes (Bird et al., 1978; Keizer, et al., 2010). Therefore, we included beta suppression in our gammaenhancing neurofeedback protocol, to investigate just gamma-band activity self-regulation. The width of the gamma and beta bands were made identical, to prevent possible effects of a bandwidth difference in the gamma and beta band (Keizer et al., 2010).
The neurofeedback paradigm was generated by using the BioGraph Infiniti Multimedia Biofeedback Software (Thought Technology Ltd, Montreal, QC). During the neurofeedback training procedure, two thresholds were implemented, one applied on the gamma and the other on the beta signal peak-to-peak amplitude. The gamma and beta threshold was set automatically to the value that the gamma and beta signal peak-to-peak amplitude surpassed above and below it respectively, in $60 \%$ of the time during the preceding 30 seconds window. Whenever the gamma peak-to-peak amplitude exceeded above the gamma threshold and at the same time, the beta peak-topeak amplitude exceeded below the beta threshold, for at least $250 \mathrm{~ms}$, the subject received some visual and auditory rewards represented on a 17-inch computer screen 1 meter away from her. The subjects were only instructed to be mentally focused and physically relaxed during neurofeedback training. They could utilize any mental strategy they wanted to gain as more rewards as possible.

During neurofeedback training, two online filters over the frequency ranges of $36-44 \mathrm{~Hz}$ and $12-20 \mathrm{~Hz}$ were applied to the recorded Oz-EEG signal to extract gamma and beta activity, respectively. Movement artifacts caused by muscle contraction (EMG) is a significant source of noise at these frequency ranges, especially over the gamma band, which can cause a false-positive error in neurofeedback training. To ensure that gamma and beta activity is properly extracted and used in neurofeedback training, we first asked all subjects to sit completely relaxed in a comfortable chair throughout the neurofeedback experiments, with no stress or extra muscle contraction or movement. We also carefully monitored the behavior of all subjects during neurofeedback training, to exclude signal intervals identified as being contaminated 
with artifacts, for further analysis. The second approach we used to prevent augmentation of the gamma or beta signal by muscle artifacts was to apply an online artifact rejection threshold, which is a nonlinear filter, on the EEG signal during training. The artifact rejection threshold was identified for each subject individually, at the beginning of each training session. For this reason, the subjects were asked to perform tasks such as blinking, or contraction of the facial muscles, while the experimenter set the artifact threshold to the value on the 50-64 Hz filtered EEG signal peak-to-peak amplitude that the amplitude surpassed above it during the contraction tasks. The subjects were not rewarded when the amplitude exceeded above the defined artifact threshold during the training, even if the gamma and the beta amplitudes satisfied the neurofeedback training goal. Other techniques, such as adaptive filters, can also be used to remove artifacts from EEG signals, which are more computationally expensive and was not adopted in this study.

\subsection{Data analysis}

The subjects were physically relaxed and still during the EEG signals recording. Therefore, only a $0.2-48 \mathrm{~Hz}$ band-pass Butterworth filter and then visual inspection was used for artifact rejection. The absolute power of the theta $(4-8 \mathrm{~Hz})$, alpha $(8-12 \mathrm{~Hz})$, beta $(12-20 \mathrm{~Hz})$, and gamma $(36-48 \mathrm{~Hz})$ frequency bands were extracted from pre-test baseline EEG signals to be investigated as neurophysiological predictors. We also explored any possible association between participants' neurofeedback performance and both fluid intelligence and mental strategies used by them during training. To investigate the effects of neurofeedback training on subjects' brain activity, gamma absolute power of pretraining baseline, and during training EEG signals were extracted. EEG signals' frequency bands' absolute power was estimated using the Welch algorithm and MATLAB R2013a software (MathWorks, Inc., Natick, MA, USA).

To identify responders and non-responders to neurofeedback training, the mean gamma absolute power change from pretraining to the training of each session was calculated for each subject. A subject was assigned to the responders' group if the mean gamma power change was positive for both over 8 sessions and each session separately, which means that gamma power during each training session was controlled and increased. Five subjects reached the criterion and categorized as responders. In contrast, a subject was assigned to the non-responders group if the mean gamma power change over 8 sessions was negative. Two subjects showing a decrease in gamma power over 5 sessions of 8 , were categorized as non-responders. The mean gamma power change of the last subject was positive over 8 sessions but negative for 2 sessions of the total 8 sessions. Considering that one of these sessions was the last training one, we categorized this subject as the poor responder.

In this study, we employed 4 types of statistical analysis, using IBM SPSS V. 21 (SPSS, Inc., Chicago, IL, USA): 1. The Kolmogorov-Smirnov (K-S) test to check for normal distribution of the variables; 2 . The paired samples $t$ test, Wilcoxon and Mann-Whitney $U$ tests to examine significant differences; 3. The Pearson correlation analysis to evaluate significant statistical relationships; 4. The simple linear and quadratic regression analysis to identify statistical trends.

\section{Results}

\subsection{Neurofeedback Performance: Responders vs. Non-responders}

Subjects' age and Body Mass Index (BMI) were both normally distributed conformed by the K-S result (age: $\mathrm{z}=0.430$; BMI: $\mathrm{z}=0.518 ; \mathrm{P}>0.05)$, with the mean of $24.46 \pm 2.35$ years and $20.65 \pm 2.62 \mathrm{~kg} / \mathrm{m}^{2}$, respectively (Table 3). Based on the Mann-Whitney $U$ test, there was no significant difference between the age of the two groups of responders and non-responders, while a significant difference was found in BMI between the two groups (age: $\mathrm{z}=-0.67 ; \mathrm{P}>0.05$; BMI: $\mathrm{z}=-2.00 ; \mathrm{P}<0.05$ ) (Table 3). Based on pre-test and pretraining questionnaires, there were no side effects of neurofeedback training on subjects and all were physically and mentally prepared for participating in the experiments. Examining posttraining questionnaires revealed the very motivated and extremely motivated levels for all subjects in all sessions.

Using the paired t test, a significant increase in gamma power from pretraining to training across 8 sessions was found for each responder $(\mathrm{P}<0.01)$, in the absence of a significant difference for each non-responder $(\mathrm{P}>0.05)$. The poor responder subject showed an increase in gamma power over 8 sessions at the significance level of $\mathrm{P}=0.075$, suggesting that she could not control her gamma activity completely. The Wilcoxon test revealed a significant difference between mean gamma power of pretraining and training across 8 sessions for responders (including the poor responder) $(\mathrm{z}=-2.20 ; \mathrm{P}<0.05)$, while no significant difference for non-responders was achieved $(\mathrm{z}=-1.34 ; \mathrm{P}>0.05)$ (Figure $2 \&$ Table 3$)$. 


\section{Responders vs. Non-responders Performance}

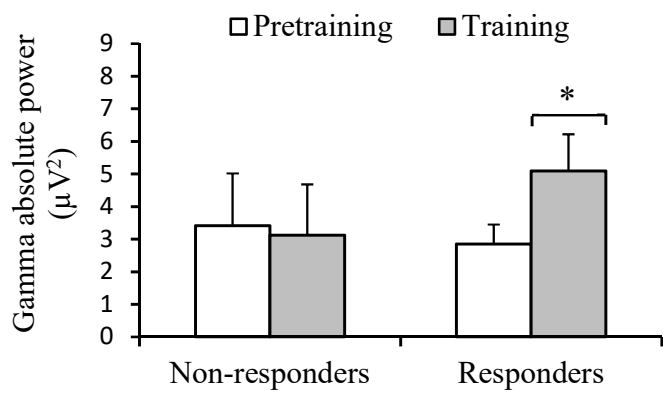

NEUR SCIENCE

Figure 2. Mean gamma absolute power over 8 sessions. Unlike non-responders, responders could significantly increase their gamma activity over training sessions. Error bars represent the standard error of the mean. The asterisk indicates a significance level of $\mathrm{P}<0.05$.

\subsection{Psychological factor: Fluid intelligence}

The subjects' Intelligence Quotient (IQ) reflecting the fluid intelligence was normally distributed confirmed by the K-S $(\mathrm{z}=0.512 ; \mathrm{P}>0.05)$, with the mean value of $103.50 \pm 22.58$ (Table 3). Using the Mann-Whitney U test, a significant difference in IQ was found between the responders and non-responders groups $(\mathrm{z}=-2.01 ; \mathrm{P}<0.05)$ (Table 3). As seen in Figure 3A, responders have a significantly higher IQ than non-responders (responders' mean percentage correct: $51.33 \pm 8.64$; non-responders' mean percentage correct: $33.00 \pm 7.07$ ). The Pearson test revealed a significant positive correlation between subjects' pre-existing fluid intelligence and mean gamma power change from pretraining to training over 8 neurofeedback sessions $(\mathrm{r}=0.75 ; \mathrm{n}=8 ; \mathrm{P}<0.05)$ (Figure $3 \mathrm{~B}$ ). This effect reflects more gamma power increase and more success in neurofeedback for people with higher IQ.
The results indicate that fluid intelligence has predictive value for estimating gamma neurofeedback performance of people, and can be used for classifying subjects as responders or non-responders before training. Based on the mathematical relationship between intelligence and gamma power change, the user's mean gamma power increase during neurofeedback sessions can be predicted. However, based on the Pearson analysis results, the fluid intelligence factor is only accounted for $56 \%$ of the variance $\left(\mathrm{R}^{2}\right)$ in mean gamma power increase suggesting that other influencing factors contribute to self-regulating brain gamma activity. Confirming this, the IQ of the poor responder subject was higher than the average IQ of all subjects, but she could not successfully make a significant increase in her brain gamma power over neurofeedback sessions.

\subsection{Neurophysiological factor: Brain neural ativity}

To identify potential neurophysiological predictors for gamma neurofeedback performance, we studied statistical relationships between gamma, beta, alpha, and theta absolute power extracted from pre-test EEG signals of two cognitive and relaxed conditions and mean gamma power change over 8 training sessions, using the Pearson correlation. The results showed a significant strong positive correlation between cognitive task gamma absolute power and mean gamma power change of a group of 6 subjects, excluding one non-responder and the poor responder subjects as outliers $(\mathrm{r}=0.88 ; \mathrm{n}=6 ; \mathrm{P}=0.02)$ (Figure 4A). Another significant correlation was found between at rest alpha absolute power and mean gamma power change of a group of 7 subjects, at a significance level of $\mathrm{P}=0.06$ ( $\mathrm{r}=-0.72 ; \mathrm{n}=7 ; \mathrm{P}=0.06$ ) (Figure 4B). No significant correlations were found between the mean gamma power change and any of the other frequency bands' powers $(\mathrm{P}>0.05)$.
A)

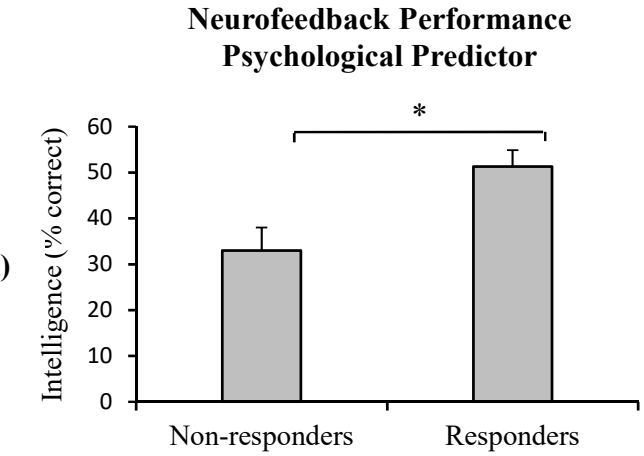

Neurofeedback Performance Psychological Predictor

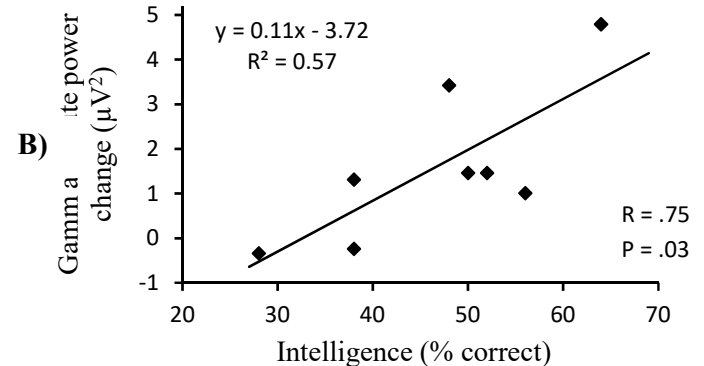

NEUR SCIENCE

Figure 3. Fluid intelligence effects on gamma-enhancing neurofeedback performance A: Responders' IQs are significantly higher than non-responders' $(\mathrm{P}<0.05)$; B: A significant positive correlation between fluid
intelligence and mean gamma power increase of all subjects over 8 neurofeedback sessions. The solid line results from a linear regression. 
Neurofeedback Performance Neurophysiological Predictor

A)

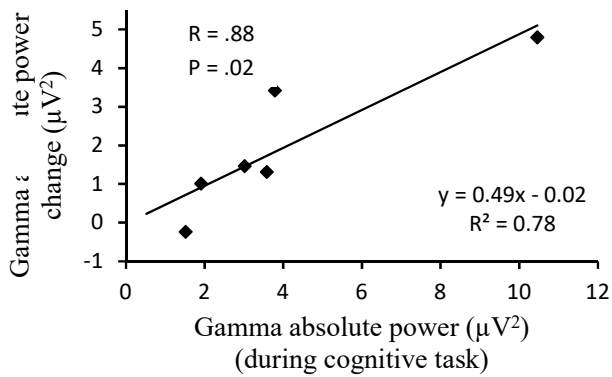

B)

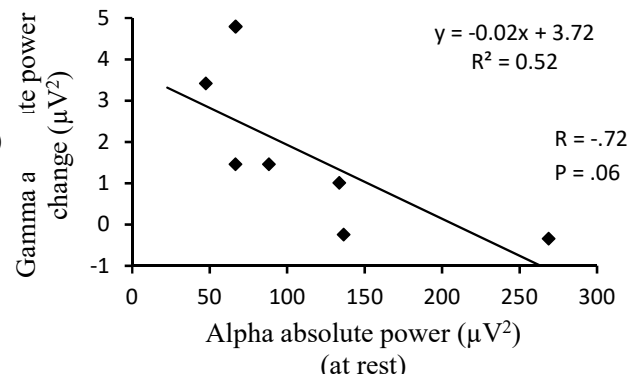

NEUR:SCIENCE

Figure 4. The statistical relationship between brain neuronal activity and gamma-enhancing neurofeedback performance

A: A significant positive correlation between cognitive task gamma absolute power and mean gamma power change of subjects over 8 neurofeedback sessions; B: A significant negative correlation between alpha absolute power during rest and gamma power change of subjects over 8 neurofeedback sessions. Solid lines result from a linear regression.

\subsection{Mental strategy: Conscious vs. Unconscious learning}

We studied posttraining questionnaires to investigate the effects of different mental strategies on gamma-enhancing neurofeedback performance. In self-reports, the subjects had written down the strategies they used during neurofeedback sessions to control their gamma activity and the effect of each strategy. Based on the subjects' descriptions, successful strategies were related to "focus on different parts of feedback image", "concentration on image variations", "predicting next feedback image", "rapid thinking and mental activity", and "no strategy". The following results have been extracted from self-reports:
- Different strategies were used by most subjects, especially in initial sessions. A distinct strategy necessarily did not have the same effects on the performance of different subjects. For example, one strategy might prove successful for one subject, but not for the rest.

- Using a successful strategy repeatedly did not lead to success again. Accordingly, after a while of using an effective strategy, one was forced to change the strategy to achieve favorable results.

- The intense mental effort resulted in less success.
Neurofeedback Conscious vs.

\section{Unconscious Learning}

A)

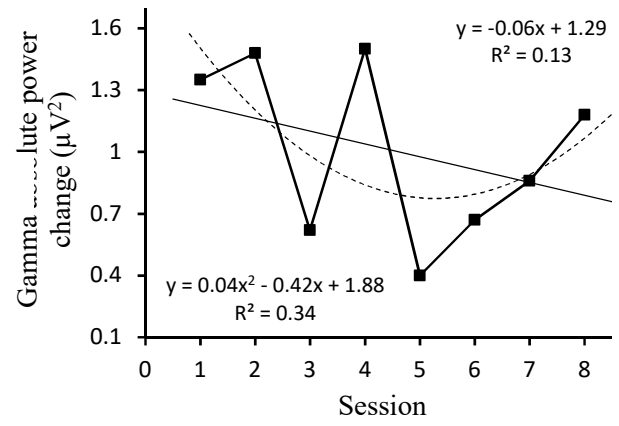

B)

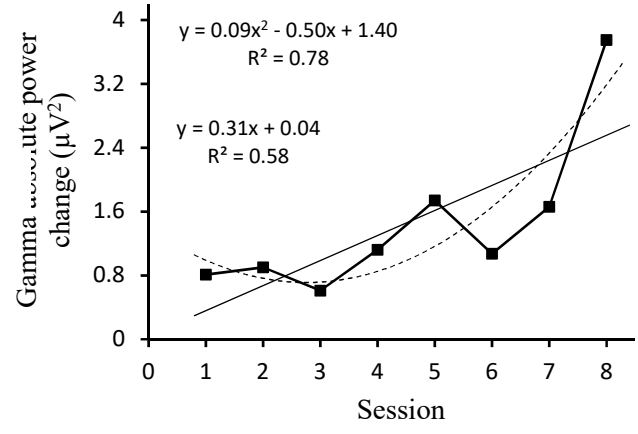

Figure 5. Gamma's absolute power change over 8 neurofeedback sessions

NEUR:SCIENCE

A: Conscious learning curve for one of the subjects using specific mental strategies during neurofeedback training. Neither linear nor quadratic trends are significant; B: Unconscious learning curve for the subject using no specific mental strategy during neurofeedback training. Both linear and quadratic trends are significant, while the quadratic trend line shows better fitting. Solid lines indicate a linear trend and dashed lines quadratic trend. 
- Most subjects could control their gamma activity without using any specific mental strategy in the final sessions. Subjects reported this condition as "I didn't do any specific mental task", "My mind was not involved in anything", and "I didn't think at all and just focused on purpose". Because of less mental effort and fatigue, they found this condition more comfortable and desirable.

- One subject did not use any specific strategy during any of the sessions. She often felt that she was not involved in the training process and could not establish the relationship between her mental states and feedback variations. The subject belonged to the responders' group and was the only one who learned neurofeedback unconsciously. Other subjects tried to learn neurofeedback consciously by trying to find relationships between their mental states and the feedback status.

To investigate gamma self-regulation conscious and unconscious learning in detail, individual linear and quadratic trends as an indicator of learning were calculated on the mean gamma power changes from pretraining to training across 8 sessions, utilizing regression analysis. Both linear $\left(\mathrm{F}_{1,6}=8.49 ; \mathrm{P}=0.03 ; \mathrm{R}^{2}=0.59\right)$ and quadratic $\left(\mathrm{F}_{2,5}=8.74 ; \mathrm{P}=0.02 ; \mathrm{R}^{2}=0.78\right)$ trends proved significant for the no strategy subject (unconscious learner), but not for any other subjects (conscious learners) $(\mathrm{P}>0.05$ ). Learning curves for one of the conscious learners and the unconscious learner are depicted in Figure 5A and B, respectively. The results suggest that using no strategy during gamma neurofeedback training results in better learning through a significant increase in gamma power from session to session. Higher $\mathrm{R}^{2}$ of the quadratic trend line $(0.78)$ compared to the linear trend line $(0.58)$ for the no strategy learning curve indicated better fitting for the quadratic model, suggesting an increase in gamma selfregulation learning rate in the final sessions.

\section{Discussion}

To solve the BCI-illiteracy problem in neurofeedback applications, previous studies proposed some psychological/ neuropsychological (Burde \& Blankertz, 2006; Daum et al., 1993; Hammer et al., 2012; Nijboer et al., 2010; Witte et al., 2013), neuroanatomical (Enriquez-Geppert et al., 2013; Halder et al., 2013), and neurophysiological (Blankertz et al., 2010) factors besides neurofeedback initial performance (Kübler et al., 2004; Neumann \& Birbaumer, 2003; Weber et al., 2011) as the predictor of individuals' neurofeedback trainability. The mental strategy used by an individual during a neurofeedback session is another factor that influences on gaining brain self-control (Angelakis et al., 2007; Birbaumer et al., 2013; Kober et al., 2013; Nan et al., 2012; Neumann \& Birbaumer, 2003; Rubik, 2011; Witte et al., 2013). The percentage of non-responders to neurofeedback training of different frequency bands have been reported as $10 \%-50 \%$, including healthy and unhealthy subjects (Blankertz et al., 2010; Drechsler et al., 2007; Hammer et al., 2012; Hanslmayr et al., 2005; Huster et al., 2013; Kober et al., 2013; Lubar et al., 1995; Neumann \& Birbaumer, 2003; Weber et al. 2011). Gamma neurofeedback illiteracy has rarely been investigated in studies. In the current study, $25 \%$ of participants were identified as non-responders to gamma neurofeedback. The proportion of non-responders identified in each study depends on the defined performance criterion. Because of the non-uniform increase in the gamma power over training sessions of participants in this study, we used the power increase in all sessions as the performance criterion. Based on this criterion, the two identified groups of responders and non-responders were statistically significantly different in mean gamma power increase over training sessions.

Our results indicated that individuals who have higher fluid intelligence could perform better in learning gamma self-control through neurofeedback. This finding confirms the role of fluid intelligence in identifying new relationships and skills acquisition (Valentin Kvist \& Gustafsson, 2008; Watkins, Lei, \& Canivez, 2007). In contrast to our results, Keizer et al. (2010) did not find any significant correlation between individuals' pre-test fluid intelligence and gamma power change through neurofeedback. They examined gamma power increase from pre-test to posttest at rest condition, but we instead focused on gamma self-regulation during neurofeedback, directly. Drechsler et al. (2007) reported no significant correlation between IQ measures and the selfregulation performance of participants. They employed a short form of the German WISC III as the IQ test, containing both fluid and crystallized intelligence measures that were not comparable with our study in which only fluid intelligence was tested.

Our findings also suggested that gamma power during cognitive task and alpha power at rest might be a predictor of gamma neurofeedback performance. Supporting our results, other studies have also reported a significant correlation between neurofeedback performance and brain neural activities measured before training (Blankertz et al., 2010; Nan et al., 2015; Wan et al., 2014). Assessing frequency bands' powers as the predictor of neurofeedback performance has some limitations. Brain potentials are affected by transient factors such as the current level of fatigue or attention, or mental states. Hence, it seems more reliable to estimate each neuro- 
feedback session performance based on brain potentials measured before the same session or more complex factors with less dependency on mental states.

Studying posttraining self-reports of participants about the effectiveness of strategies they used during neurofeedback training revealed that:

Visual strategies involved in image perception and memory restoration are possibly successful strategies for individual gamma-enhancing neurofeedback. This finding is in accordance with previous findings implicated that brain neural synchronization in the gamma band is associated with visual objects perception, visual working memory, and also short-term and long-term memory (Elliott \& Müller, 1998; Keizer, et al., 2010; Sams, 1995; TallonBaudry \& Bertrand, 1999; Tallon-Baudry et al., 1998).

Although participants in initial sessions have used different strategies, they found that conducting later sessions with no intense mental effort and specific strategy was more successful and comfortable. This finding is in line with the theory that neurofeedback learning can be viewed as skill learning (Birbaumer et al., 2013; Kober et al., 2013); brain self-regulation is originally based on trialand-error. Then, the learned self-regulation skill stores in the implicit memory and its retrieval requires no conscious and effortful search.

Our results showed that unconscious learners had better performance compared to other conscious learners. Moreover, both intense mental effort and repeating successful strategies by participants failed to gain appropriate brain self-control. Probably employing conscious mental strategies by participants, especially in later sessions, overload cognitive resources, which impedes complete automatization of self-regulation skill acquisition (Kober et al., 2013; Neumann \& Birbaumer, 2003). Accordingly, although the IQ of the poor responder participant was above the average IQ of all participants, her poor performance might be due to spending too much effort in using different types of strategies even in final sessions (mentioned by her in her self-report). Supporting this finding, the effectiveness of using no conscious mental strategy in neurofeedback learning of other frequency bands (e.g. SCP, SMR, and alpha) have also been demonstrated (Angelakis et al., 2007; Birbaumer et al., 2013; Kober et al., 2013; Neumann \& Birbaumer, 2003; Witte et al., 2013).

Another finding of this study was that a distinct strategy had different effects on the performance of different individuals. Nan et al. (2012) also mentioned this finding in their alpha neurofeedback study. Regarding this finding and the efficacy of unconscious neurofeedback learning, we support the conclusion of other studies (Birbaumer et al., 2013; Kober et al., 2013; Neumann \& Birbaumer, 2003; Witte et al., 2013) about providing neurofeedback participants with no explicit limiting instructions before training and just ask them to be physically relaxed while mentally focused without any hard mental efforts.

One advantage of this study compared to previous studies is that we assessed personal factors in relation to neurofeedback performance over 8 training sessions, while most previous studies just investigated neurofeedback performance in one training session. Skill learning occurred through time. Moreover, transient factors such as the current level of fatigue or attention influence both brain potentials and learning in one session. Therefore, assessing neurofeedback overall performance over several training sessions seems more reliable in such studies. Another advantage of the current study to other studies predicting neurofeedback later performance from initial performance is that the predictive factors introduced here for estimating neurofeedback trainability can be measured before the neurofeedback training course. In this way, we can avoid conducting any initial neurofeedback sessions with the risk of useless temporal and financial costs for non-responders. Second, the steadiness or variability of the participants' learning rate should be considered in studies focusing on the predictive value of neurofeedback early performance, which was rarely investigated before (Kübler et al., 2004). One important limitation of our study is the small number of participants. Some results have been reported on two non-responder subjects or only one unconscious learner. Another limitation was that our participants were only females. Hence, our findings should be considered preliminary and their utilization in clinical and experimental setups needs further validation.

\section{Conclusion}

In summary, in this study, we introduce three predictors for estimating gamma-enhancing neurofeedback trainability of individuals: 1. Fluid intelligence; 2. Gammaband power of EEG during a cognitive task; and 3. Alpha band power of EEG at eyes-closed rest. According to our results, a significant number of individuals cannot control their brain activity through gamma neurofeedback. The proposed psychological and neurophysiological predictors in this study can be used for classifying subjects as responders or non-responders before gamma neurofeedback training, avoiding to conduct any useless training sessions. Another important and applicable finding of this research is that employing no specific mental 
effort and conscious strategy by users during gamma neurofeedback (unconscious learning) leads to more success in gamma self-regulation learning.

\section{Ethical Considerations}

\section{Compliance with ethical guidelines}

All ethical principles are considered in this article. The participants were informed about the purpose of the research and its implementation stages; they were also assured about the confidentiality of their information; moreover, they were free to leave the study whenever they wished, and if desired, the research results would be available to them.

\section{Funding}

This research did not receive any grant from funding agencies in the public, commercial, or non-profit sectors.

\section{Authors' contributions}

Both authors were equally contributed in preparing this article.

\section{Conflict of interest}

The authors declared no conflict of interest.

\section{References}

Angelakis, E., Stathopoulou, S., Frymiare, J. L., Green, D. L., Lubar, J. F., \& Kounios, J. (2007). EEG neurofeedback: A brief overview and an example of peak alpha frequency training for cognitive enhancement in the elderly. The Clinical Neuropsychologist, 21(1), 110-29. [DOI:10.1080/13854040600744839] [PMID]

Arns, M., Kleinnijenhuis, M., Fallahpour, K., \& Breteler, R. (2008). Golf performance enhancement and real-life neurofeedback training using personalized event-locked EEG profiles. Journal of Neurotherapy, 11(4), 11-8. [DOI:10.1080/10874200802149656]

Başar-Eroglu, C., Strüber, D., Schürmann, M., Stadler, M., \& Başar, E. (1996). Gamma-band responses in the brain: A short review of psychophysiological correlates and functional significance. International Journal of Psychophysiology, 24(1-2), 10112. [DOI:10.1016/S0167-8760(96)00051-7]

Berner, I., Schabus, M., Wienerroither, T., \& Klimesch, W. (2006). The significance of sigma neurofeedback training on sleep spindles and aspects of declarative memory. Applied Psychophysiology and Biofeedback, 31(2), 97-114. [DOI:10.1007/s10484006-9013-7] [PMID] [PMCID]
Birbaumer, N., Ruiz, S., \& Sitaram, R. (2013). Learned regulation of brain metabolism. Trends in Cognitive Sciences, 17(6), 295302. [DOI:10.1016/j.tics.2013.04.009] [PMID]

Bird, B. L., Newton, F. A., Sheer, D. E., \& Ford, M. (1978). Biofeedback training of 40-Hz EEG in humans. Biofeedback and Self-Regulation, 3(1), 1-11. [DOI:10.1007/BF00998559]

Blankertz, B., Sannelli, C., Halder, S., Hammer, E. M., Kübler, A., \& Müller, K.-R., et al. (2010). Neurophysiological predictor of SMR-based BCI performance. NeuroImage, 51(4), 1303-9. [DOI:10.1016/j.neuroimage.2010.03.022] [PMID]

Burde, W., \& Blankertz, B. (2006). Is the locus of control of reinforcement a predictor of brain-computer interface performance? Paper presented at the Proceedings of the $3^{\text {rd }}$ International BrainComputer Interface Workshop and Training Course. https:// graz.pure.elsevier.com/en/publications/proceedingsof-the-3rd-international-brain-computer-interface-wor

Cattell, R. B., Krug, S. E., \& Barton, K. (1973). Technical supplement for the Culture Fair Intelligence Tests, Scales 2 and 3 Champaign, IL: Institute for Personality and Ability Testing.

Daum, I., Rockstroh, B., Birbaumer, N., Elbert, T., Canavan, A., \& Lutzenberger, W. (1993). Behavioural treatment of slow cortical potentials in intractable epilepsy: Neuropsychological predictors of outcome. Journal of Neurology, Neurosurgery $\mathcal{E}$ Psychiatry, 56(1), 94-7. [DOI:10.1136/jnnp.56.1.94] [PMID] [PMCID]

Drechsler, R., Straub, M., Doehnert, M., Heinrich, H., Steinhausen, H. C., \& Brandeis, D. (2007). Controlled evaluation of a neurofeedback training of slow cortical potentials in children with Attention Deficit/Hyperactivity Disorder (ADHD). Behavioral and Brain Functions, 3(1), 35. [DOI:10.1186/1744-90813-35] [PMID] [PMCID]

Egner, T., \& Gruzelier, J. H. (2003). Ecological validity of neurofeedback: Modulation of slow wave EEG enhances musical performance. NeuroReport, 14(9), 1221-4. [DOI:10.1097/00001756-200307010-00006] [PMID]

Egner, T., \& Gruzelier, J. H. (2004). EEG Biofeedback of low beta band components: Frequency-specific effects on variables of attention and event-related brain potentials. Clinical Neurophysiology, 115(1), 131-9. [DOI:10.1016/S1388-2457(03)00353-5]

Elliott, M. A., \& Müller, H. J. (1998). Synchronous Information Presented in 40-HZ Flicker Enhances Visual Feature Binding. Psychological Science, 9(4), 277-83. [DOI:10.1111/14679280.00055]

Enriquez-Geppert, S., Huster, R. J., Scharfenort, R., Mokom, Z., Figge, C., Zimmermann, J., \& Herrmann, C. S. (2013). The morphology of midcingulate cortex predicts frontal-midline theta neurofeedback success. Frontiers in Human Neuroscience, 7, 453. [DOI:10.3389/fnhum.2013.00453] [PMID] [PMCID]

Gevensleben, H., Holl, B., Albrecht, B., Vogel, C., Schlamp, D. \& Kratz, O., et al. (2009). Is neurofeedback an efficacious treatment for ADHD? A randomised controlled clinical trial. Journal of Child Psychology and Psychiatry, 50(7), 780-9. [DOI:10.1111/j.1469-7610.2008.02033.x] [PMID]

Gruzelier, J. (2000). Self regulation of electrocortical activity in schizophrenia and schizotypy: A review. Clinical EEG and Neuroscience, 31(1), 23-9. [DOI:10.1177/155005940003100108] [PMID] 
Halder, S., Varkuti, B., Bogdan, M., Kübler, A., Rosenstiel, W., Sitaram, R., \& Birbaumer, N. (2013). Prediction of braincomputer interface aptitude from individual brain structure. Frontiers in Human Neuroscience, 7, 105. [DOI:10.3389/fnhum.2013.00105] [PMID] [PMCID]

Hammer, E. M., Halder, S., Blankertz, B., Sannelli, C., Dickhaus, T., \& Kleih, S., et al. (2012). Psychological predictors of SMR-BCI performance. Biological Psychology, 89(1), 80-6. [DOI:10.1016/j.biopsycho.2011.09.006] [PMID]

Hammond, D. C. (2000). What do we know about $40 \mathrm{~Hz}$ activity and the tunction it serves? Journal of Neurotherapy: Investigations in Neuromodulation, Neurofeedback and Applied Neuroscience, 4(2), 95-104. [DOI:10.1300/J184v04n02_10]

Hanslmayr, S., Sauseng, P., Doppelmayr, M., Schabus, M., \& Klimesch, W. (2005). Increasing individual upper alpha power by neurofeedback improves cognitive performance in human subjects. Applied Psychophysiology and Biofeedback, 30(1), 1-10. [DOI:10.1007/s10484-005-2169-8] [PMID]

Hommel, B. (1998). Event files: Evidence for automatic integration of stimulus-response episodes. Visual Cognition, 5(1/2), 183-216. [DOI:10.1080/713756773]

Huster, R. J., Mokom, Z. N., Enriquez-Geppert, S., \& Herrmann, C. S. (2013). Brain-computer interfaces for EEG neurofeedback: Peculiarities and solutions. International Journal of Psychophysiology, 91(1), 36-45. [DOI:10.1016/j.ijpsycho.2013.08.011] [PMID]

Keizer, A. W., Verment, R. S., \& Hommel, B. (2010). Enhancing cognitive control through neurofeedback: A role of gammaband activity in managing episodic retrieval. NeuroImage, 49(4), 3404-13. [DOI:10.1016/j.neuroimage.2009.11.023] [PMID]

Keizer, A. W., Verschoor, M., Verment, R. S., \& Hommel, B. (2010). The effect of gamma enhancing neurofeedback on the control of feature bindings and intelligence measures. International Journal of Psychophysiology, 75(1), 25-32. [DOI:10.1016/j. ijpsycho.2009.10.011] [PMID]

Khodakarami, Z., \& Firoozabadi, S. M. P. (2014). [Self-regulation of brain gamma band activity through neurofeedback and its effects on visual feature bindings in healthy female students (Persian)]. Koomesh, 16(1), 36-45. http:/ / koomeshjournal.semums.ac.ir/article-1-2298-fa.html

Kober, S. E., Witte, M., Ninaus, M., Neuper, C., \& Wood, G. (2013). Learning to modulate one's own brain activity: The effect of spontaneous mental strategies. Frontiers in Human Neuroscience, 7, 695. [DOI:10.3389/fnhum.2013.00695] [PMID] [PMCID]

Kübler, A., Blankertz, B., Müller, K., \& Neuper, C. (2011). A model of BCI control. Paper presented at the Proceedings of the $5^{\text {th }}$ International Brain-Computer Interface Conference. https:// graz.pure.elsevier.com/en/publications/proceedings-ofthe-5th-international-brain-computer-interface-con

Kübler, A., Neumann, N., Wilhelm, B., Hinterberger, T., \& Birbaumer, N. (2004). Predictability of brain-computer communication. Journal of Psychophysiology, 18(2-3), 121-9. [DOI:10.1027/0269-8803.18.23.121]

Landers, D. M., Petruzzello, S. J., Salazar, W., Crews, D. J., Kubitz, K. A., \& Gannon, T. L.,et al. (1991). The influence of electrocortical biofeedback on performance in pre-elite archers. Medicine \& Science in Sports \& Exercise, 23(1), 123-9. [DOI:10.1249/00005768-199101000-00018]

Leins, U., Goth, G., Hinterberger, T., Klinger, C., Rumpf, N., \& Strehl, U. (2007). Neurofeedback for children with ADHD: A comparison of SCP and theta/beta protocols. Applied Psychophysiology and Biofeedback, 32(2), 73-88. [DOI:10.1007/s10484007-9031-0] [PMID]

Lubar, J., Swartwood, M., Swartwood, J., \& O'Donnell, P. (1995). Evaluation of the effectiveness of EEG neurofeedback training for ADHD in a clinical setting as measured by changes in T.O.V.A. scores, behavioral ratings, and WISCR performance. Biofeedback and Self-Regulation, 20(1), 83-99. [DOI:10.1007/BF01712768] [PMID]

Moore, N. (2000). A review of EEG biofeedback treatment of anxiety disorders. Clinical EEG (Electroencephalography), 31(1) 1-6. [DOI:10.1177/155005940003100105] [PMID]

Nan, W., Rodrigues, J. P., Ma, J., Qu, X., Wan, F., \& Mak, P. I., et al. (2012). Individual alpha neurofeedback training effect on short term memory. International Journal of Psychophysiology, 86(1), 83-7. [DOI:10.1016/j.ijpsycho.2012.07.182] [PMID]

Nan, W., Wan, F., Vai, M. I., \& Da Rosa, A. C. (2015). Resting and initial beta amplitudes predict learning ability in beta/ theta ratio neurofeedback training in healthy young adults. Frontiers in Human Neuroscience, 9, 677. [DOI:10.3389/fnhum.2015.00677] [PMID] [PMCID]

Neumann, N., \& Birbaumer, N. (2003). Predictors of successful self control during brain-computer communication. Journal of Neurology, Neurosurgery \& Psychiatry, 74(8), 1117-21. [DOI:10.1136/jnnp.74.8.1117] [PMID] [PMCID]

Nijboer, F., Birbaumer, N., \& Kübler, A. (2010). The influence of psychological state and motivation on brain-computer interface performance in patients with amyotrophic lateral sclerosis-a longitudinal study. Frontiers in Neuroscience, 4, 55. [DOI:10.3389/fnins.2010.00055] [PMID] [PMCID]

Ninaus, M., Kober, S., Witte, M., Koschutnig, K., Neuper, C., \& Wood, G. (2015). Brain volumetry and self-regulation of brain activity relevant for neurofeedback. Biological Psychology, 110, 126-33. [DOI:10.1016/j.biopsycho.2015.07.009] [PMID]

Raymond, J., Sajid, I., Parkinson, L., \& Gruzelier, J. (2005). Biofeedback and dance performance: A preliminary investigation. Applied Psychophysiology and Biofeedback, 30(1), 6573. [DOI:10.1007/s10484-005-2175-x] [PMID]

Raymond, J., Varney, C., Parkinson, L. A., \& Gruzelier, J. H. (2005). The effects of alpha/theta neurofeedback on personality and mood. Cognitive Brain Research, 23(2-3), 287-92. [DOI:10.1016/j.cogbrainres.2004.10.023] [PMID]

Rubik, B. (2011). Neurofeedback-enhanced gamma brainwaves from the prefrontal cortical region of meditators and nonmeditators and associated subjective experiences. The Journal of Alternative and Complementary Medicine, 17(2), 109-15. [DOI:10.1089/acm.2009.0191] [PMID]

Sams, M. W. (1995). Mathematically derived frequency correlates in cerebral function: Theoretical and clinical implications for neurofeedback treatment. Journal of Neurotherapy, 1(2), 1-14. [DOI:10.1300/J184v01n02_01]

Staufenbiel, S. M., Brouwer, A. M., Keizer, A. W., \& van Wouwe, N. C. (2014). Effect of beta and gamma neurofeedback on 
memory and intelligence in the elderly. Biological Psychology, 95, 74-85. [DOI:10.1016/j.biopsycho.2013.05.020] [PMID]

Tallon-Baudry, C., \& Bertrand, O. (1999). Oscillatory gamma activity in humans and its role in object representation. Trends in Cognitive Sciences, 3(4), 151-62. [DOI:10.1016/S13646613(99)01299-1]

Tallon-Baudry, C., Bertrand, O., Peronnet, F., \& Pernier, J. (1998). Induced $\gamma$-band activity during the delay of a visual shortterm memory task in humans. The Journal of Neuroscience, 18(11), 4244-54. [DOI:10.1523/JNEUROSCI.18-11-04244.1998] [PMID] [PMCID]

Valentin Kvist, A., \& Gustafsson, J. E. (2008). The relation between fluid intelligence and the general factor as a function of cultural background: A test of Cattell's Investment theory. Intelligence, 36(5), 422-36. [DOI:10.1016/j.intell.2007.08.004]

van Boxtel, G. J. M., Denissen, A. J. M., Jäger, M., Vernon, D., Dekker, M. K. J., \& Mihajlović, V., et al. (2012). A novel selfguided approach to alpha activity training. International Journal of Psychophysiology, 83(3), 282-94. [DOI:10.1016/j. ijpsycho.2011.11.004] [PMID]

Vernon, D., Egner, T., Cooper, N., Compton, T., Neilands, C., \& Sheri, A., et al. (2003). The effect of training distinct neurofeedback protocols on aspects of cognitive performance. International Journal of Psychophysiology, 47(1), 75-85. [DOI:10.1016/ S0167-8760(02)00091-0]

Wan, F., Nan, W., Vai, M. I., \& Rosa, A. (2014). Resting alpha activity predicts learning ability in alpha neurofeedback. Frontiers in Human Neuroscience, 8, 500. [DOI:10.3389/fnhum.2014.00500] [PMID] [PMCID]

Watkins, M. W., Lei, P. W., \& Canivez, G. L. (2007). Psychometric intelligence and achievement: A cross-lagged panel analysis. Intelligence, 35(1), 59-68. [DOI:10.1016/j.intell.2006.04.005]

Weber, E., Köberl, A., Frank, S., \& Doppelmayr, M. (2011). Predicting successful learning of SMR neurofeedback in healthy participants: Methodological considerations. Applied Psychophysiology and Biofeedback, 36(1), 37-45. [DOI:10.1007/s10484010-9142-x] [PMID]

Witte, M., Kober, S. E., Ninaus, M., Neuper, C., \& Wood, G. (2013). Control beliefs can predict the ability to up-regulate sensorimotor rhythm during neurofeedback training Frontiers in Human Neuroscience, 7, 478. [DOI:10.3389/fnhum.2013.00478] [PMID] [PMCID] 